
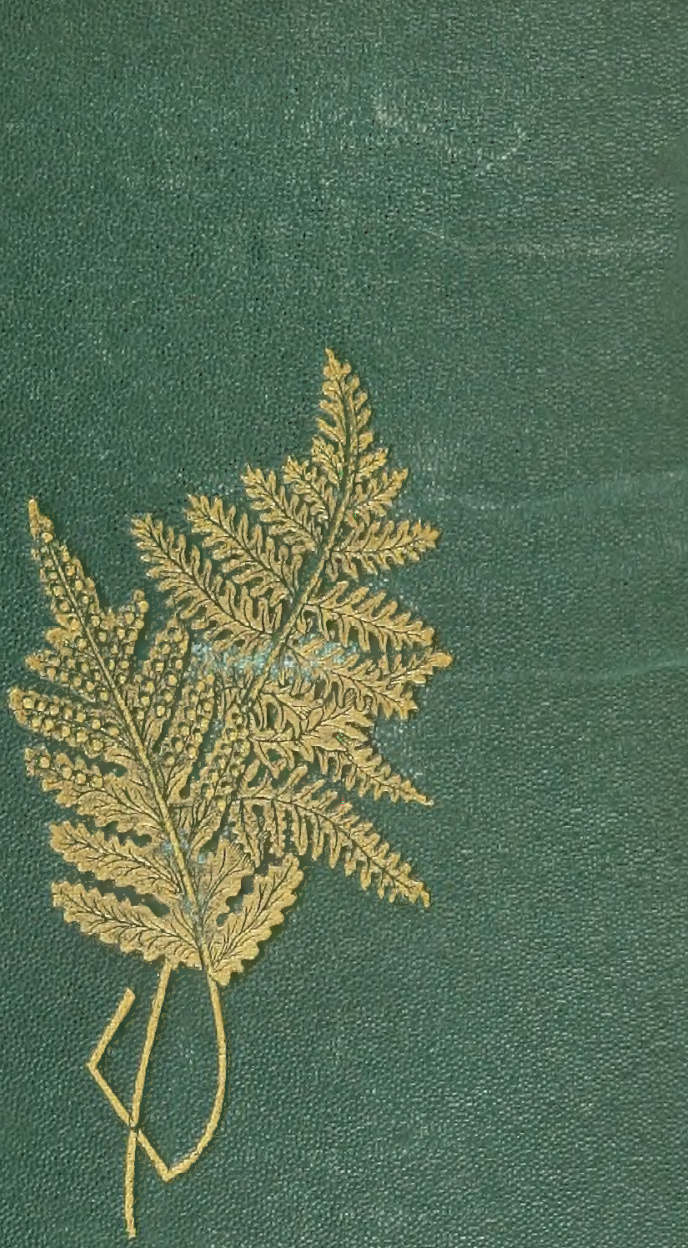

사 


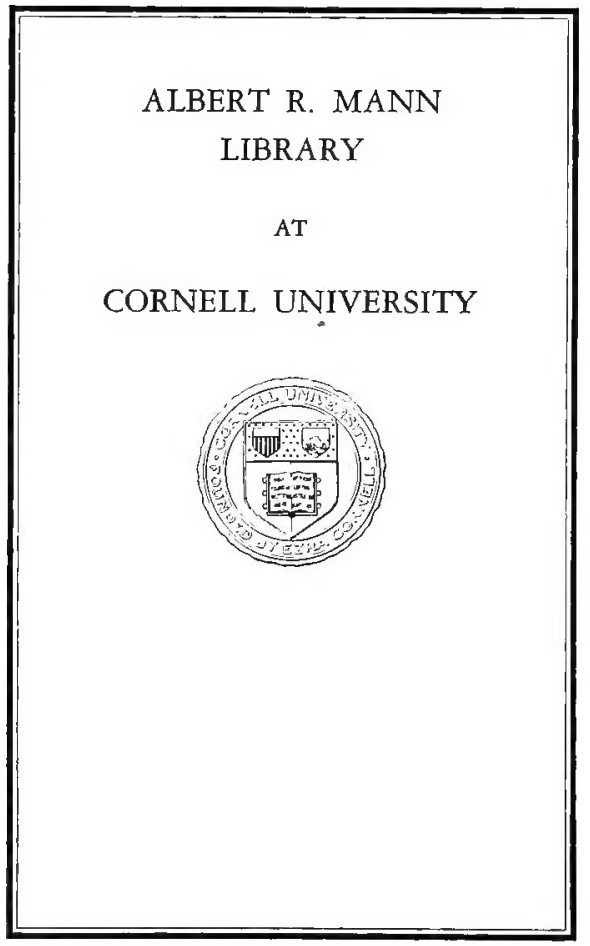




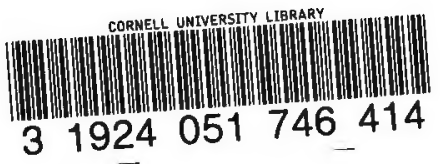

DATE DUE

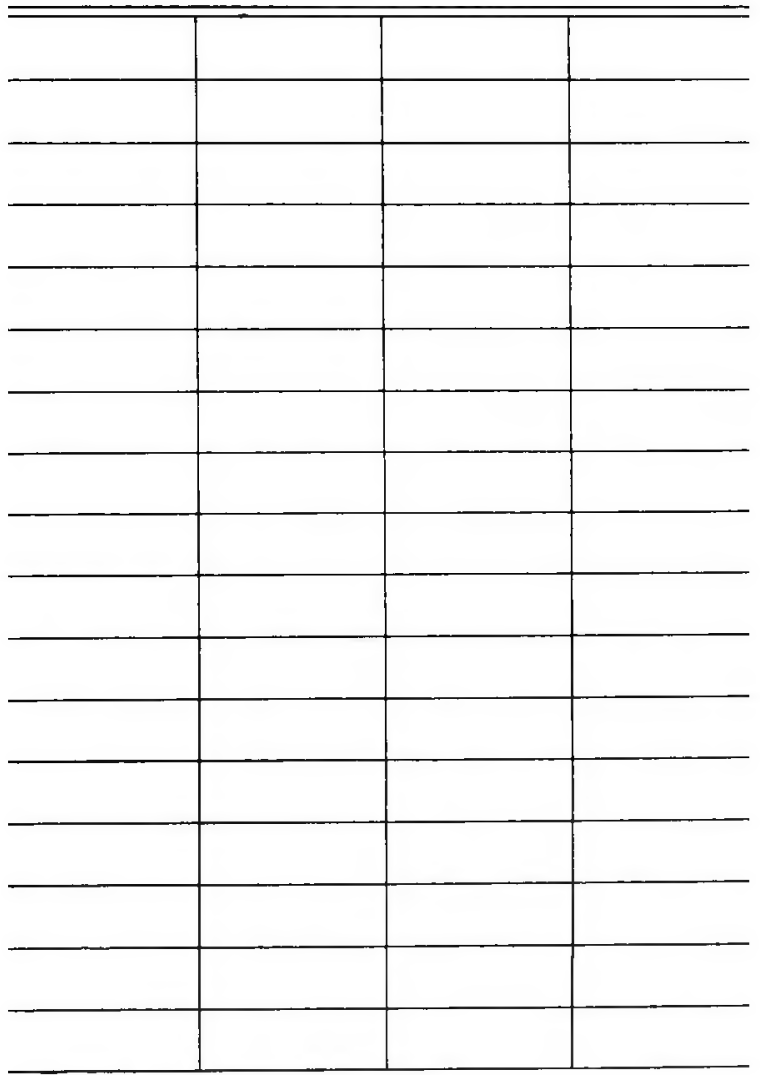

DEMCO 38-297 


\section{Cornell University Library}

The original of this book is in the Cornell University Library.

There are no known copyright restrictions in the United States on the use of the text.

http://www.archive.org/details/cu31924051746414 




HANDBOOK

DF

$\begin{array}{lllll}\mathrm{F} & \mathrm{E} & \mathrm{R} & \mathrm{N} & \mathrm{S}\end{array}$ 
LONDON: FRINRD BY SPOTTISWOODG AND CO., NEW-STRLET SQUABT AND PARLIAMFNE STRELT 


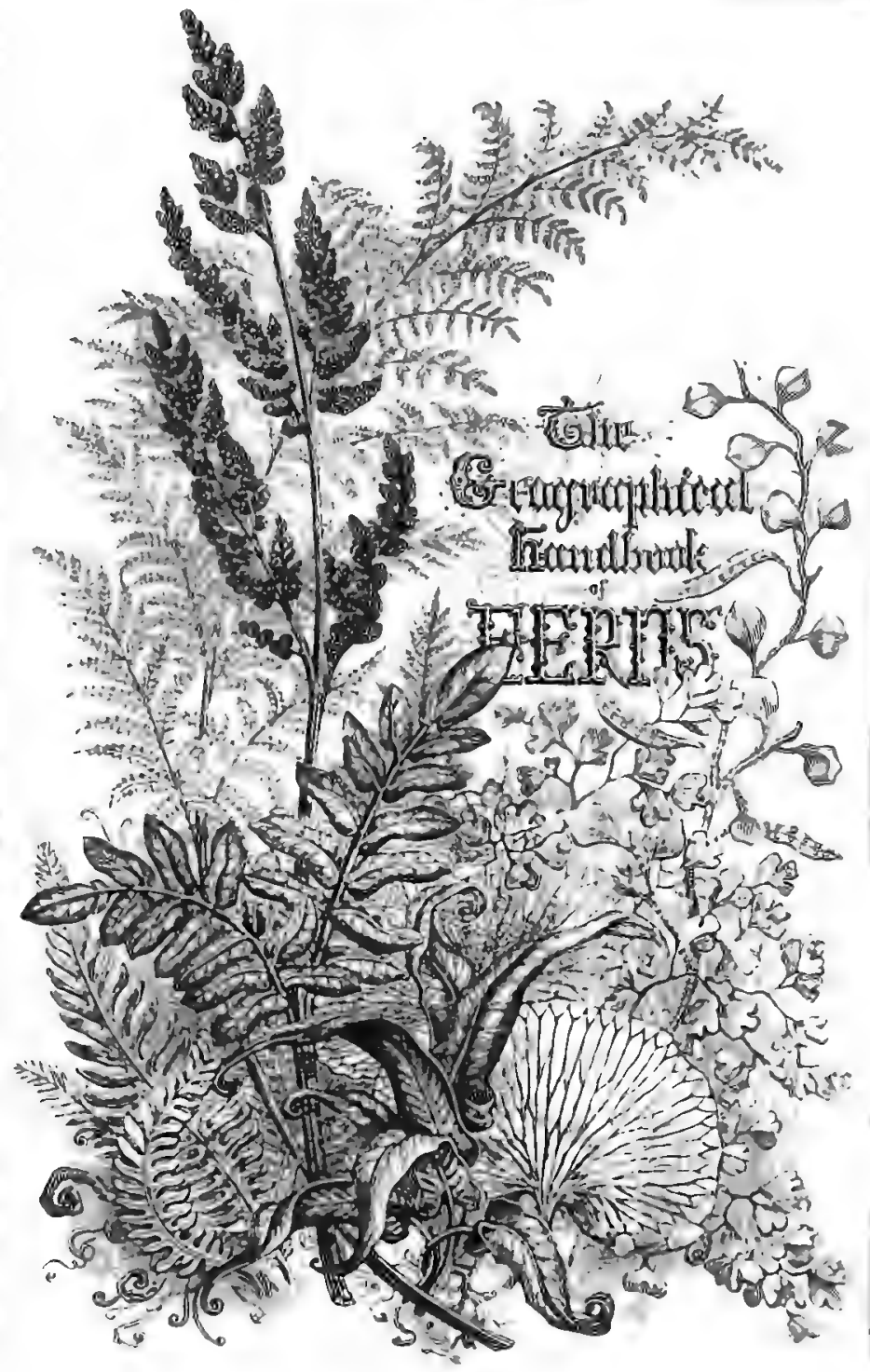

LONDON:

JOHN MURRAY, ALBEMARLE STREET, 1870 . 

A

\title{
GEOGRAPHICAL HANDBOOK
}

\author{
OF ALL THE ENOWN
}

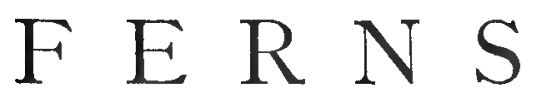

WITH TABLES TO SHOW THEIR DISTRIBUTION

\section{By K. M. LYELU}

\section{LONDON \\ JOHN MURRAY, ALBEMARLE STREET 1870}


QK

5:3

$\angle 98$

187 


\section{PREFACE}

Among the numerous works on Ferns that have hitherto appeared, there is none giving the great geographical divisions where every known species of that beautiful order has been recognised. This catalogue has accordingly been drawn up, with the hope that it may prove useful to those visiting various countries, as well as affording at a glance some idea of the genera of Ferns which prevail in certain areas. My text-book has been the 'Synopsis Filicum,' Sir William Hooker's latest work, completed by $\mathrm{Mr}$. Baker; and constant reference has been given to the more detailed localities in Hooker's 'Species Filicum,' as well as to various other works noted below.

I am under great obligation to Dr. Hooker, the Director of the Royal Gardens, Kew, and to Sir Charles Bunbury, for much kind assistance; also to Mr. M. P. Edgeworth, and Mr. J. H. Redfield of Philadelphia, the last of whom has furnished me with a valuable series of maps of North America, coloured by himself to illustrate all the localities of Ferns throughout that continent. 
I have endearoured to obtain all the most recent and correct information regarding the geographical distribution and detailed localities of Ferns; but I am aware errors must have unavoidably crept in, in the enumeration of so many names. Authors differ much regarding species, but I have confined myself to those given in the 'Synopsis Filicum,' and only quoted a few synonyms. Whenever I have obtained information from reliable sources, either in MS. or published matter, not given in the 'Synopsis Filicum,' I have cited the authorities.

The sign $\$$ denotes the only hitherto known localities. I have also made out a series of Tables at the end of the volume, to illustrate further the distribution of Ferns.

K. M. L. 


\section{CONTENTS.}

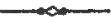

\section{EUROPE AND NORTH AFRICA.}

1. EURope Prophr $•$. . . . . . . 1

2. Algmita, Madema, Canaries, Azores . . . . . . 6

II. ASIA.

1. Normerern, Centrai, and Wegtern Asia, China and Japan । 11

2. Northesn India, including Assam, and all north of the Deccar 20

3. Southeren India, including Concay, Deccay, Orissa; Ceyjon . 33

4. Eastern Penimsula, and Archipelago. Philmpine Isles . . 48

\section{AUSTRALIA AND POLYNESIA.}

1. Tropical Austealia, New Guinea, Caroline and Solomon Istefs, Nhw Hebrides, New Catedonia . . . . . . . 68

2. Temperate Austratia, Tasmania, New Zeatand, Auchiand Istme, \&c. . . . . . . . . . 75

3. Polyngisa, Friendiy, Societr, Sandwich, Maredesa Islies, \&uc. 88

IV. AFRICA.

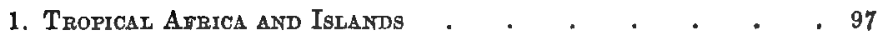

2. Cape Colony and Natal. Tristan d'Acumha . . . . 112 
viii

\section{NORTH AMERICA.}

1. Stb-Arctic Greeniand, Canada, and westerly to the Rocky Mountains . . . . . . . . . . . . 119

2. Unteded States, Bermuda . . . . . . . . . . . 121

3. California and New Mexuco. British Coldmbia, covering The slope from the Rocky Modntajns to the Pacific . . 126

\section{SOUTH AMERICA.}

1. Mexico, Panama, West India Islands . . . . . . 129

2. Venezuela, New Granada, Equador, Peru, Bolivia, Gaidapagos . 149

3. Gulana, Brazic, Paraguay, Uruguay . . . . . . 168

4. Chill, la Plata, Patagonia, Farkland Isles, Juan Fernandez . 183 


\section{The following Works have been consulted:-}

Sir William Hooker's Synopsis Filicum, completed by J. G. Baker.

$\begin{array}{ll}" & \text { Species Filicum. } \\ " & \text { Filices Exotici. } \\ " & \text { British Ferns. } \\ " & \text { Garden Ferns. } \\ & \text { Century of Ferms. } \\ & \text { Second Century of Ferns. } \\ & \text { Flora Boreali Americani. }\end{array}$

Hooker and Greville's Icones Filicum.

Hooker and Amott's Beechey's Voyage.

Dr. Joseph Hooker's Introduction to Flora Indica.

9

New Zealand Handbook.

Grisebach's Flora of the British West Indian Isles.

Thwaites' Enumeration of Ceylon Plants.

Bentham's Flora Hongkongensis.

F. Mueller's Vegetation of the Chatham Islands.

" Fragmenta Phytographiæ Australiæ.

Asa Gray's Manual of the Northern United States. Filices, by Professor D. C. Eaton.

Chapman's Flora of the Soutbern United States. Filices, by Professor D. C. Eaton.

Torrey's New York Flora.

MtCord's Paper on Canadian Ferns, in 'Canadian Naturalist.'

Kuhn's Filices Africanæ.

Milde's Filices Europæ.

Fée's Histoire des Fongères \&c. des Antilles.

Mettenius, on Ferns of India and Japan, in Miquel's Annale, \&c. 
Sir Charles Bunbury, on Botany of Madeira and Teneriffe, in 'Linnæan Society's Journal.'

Captain R. H. Beddome, Ferns of British India.

Ferns of Southern India, Madras Presidency.

H. C. Watson's List of Ferns of the Azores.

Moore's (incomplete) Index Filicum.

J.G. Baker on Geographical Distribution of Ferns, in 'Linnæan Trans.'

J. H. Redfield, Series of Maps of N. America, illustrating Fern localities. 


\section{OMISSIONS.}

PAGr 1. Onoclea Germanica, W. Hab.: N. Europe, Scandinavia to Austria.

Woodsia glabella, Br. Hab.: Norway, Tyrol, Carinthia.

W. Caucasica, J. Sm. Hab,: rocky places in the Caucasus, 1-6000 feet. $\$$.

9. Scolopendrium Homionitis, Sw, Hab.: Algiers.

1)

1)

,

1)

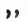

Pagn 9, line 5 from foot, for Marastæ read Marantæ.

11. Onoclea sensibilis, Linn. Hab.: Japan and Manchuria.

O. Germanica, W. Hab.. Manchuria to Altai. land. Madagascar. nada. Hab.: Mt. Chimborazo. \$.

\section{ERRATA.}

10, line 18, for lusitaricum read lnsitanicum.

17, line 5, for Lenzeanum read Leuzeanum.

27, line 5 from foot, for Lenzeanum read Leuzeanum.

48, line 4 from foot, for ramisperia read ramispina.

89, line 2, for Monzicsii read Menziesii, Hook. \& $B$.

line 32 , for tenere read tenue.

95 , last line but one, for candiformis read caudiformis.

108, line 7, for goniophlebium read Goniophlebium.

21. (After Davallia bullata), D. Lorrainei, Hance. Hab.: Malaya.

55. (After A. tenerum), A. prionurus, J. Sm. Hab.: Philippines.

84. (After A, bulbiferum), A. Colensoi, Hook, fil. Hab.: New Zea-

90. (After D. elegans), D. Fijiensis, Hook. Hab.: Fiji Islands.

107. (After P. nigritianum), P. (Goniopt.) Mascarense, Baker. Hab.

130. (After A. Mexicana), A. myosuroides, Liebm. Hab.: Mexico. \$.

154. (Before Ch. radiata), Ch. Lindigii, Mett. Hab.: New Granada.

(After Ch. radiata), Ch. propinqua, Mett. Hab. : New Gra-

161. (After P. dracónopterum), P. (Dicty.) nicotianæfolium, Baker.

174. (After Pt. denticulata), Pt. macroptera, Link. Hab. : Brazil.

15, line 8, for A. (Athyr.) crenulatum, Fries, read crenatum, Ruprecht. 



\title{
GEOGRAPHICAL HANDBOOK
}

\author{
OF \\ F E R N S.
}

\section{EUROPE AND NORTH AFRICA.}

1. EUROPE PROPER.

2. ALGERIA, MADEIRA, CANARIES, AZORES.

$\$$ denotes the only hitherto known localities.

\section{EUROPE PROPER.}

woodsia.

W. Ilvensis, $B r$. Hab. : arctic regions and high mountains in the temperate zone. Elevated mountains in the north of England and Wales. Breadalbane and Clova mountains, Scotland. Hook. Brit. Ferns, t. 8.

W. hyperborea, $B r$. Hab. : rocks, arctic and subarctic countries of Europe; rory rare in the mountainous parts of the temperate regions. Rare in Britain; found in Wales and Scotland. Hook. Brit. Ferns, t. 7.

\section{Dicksonia.}

D. Culcita, L'Hérit. Hab. : recently found at Algesiras, near Gibraltar. C. Bunbury.

\section{Fymenophyllum.}

H. Tunbridgense, Smith ( $\beta$ H. Wilsoni, Hook.). Hab. : Saxony, Normandy, Corsica, Tyrol, Italy. Britain : in Wales and Scotland; rare in the south and east of England, but more frequent in the hilly and mountain districts in the west of England.-Hook. Brit. Ferns, t. 43, $\beta$ Wilsoni. Norway, Feroe, and Britain. Abundant in the Highlands of Scotland, and in Ireland, especially about the Lakes of Killarney. Found in north of England, and Devon and Cornwall; but rare. Hook. Brit. Ferns, t. 44.

\section{Trichomanes.}

T. radicans, Swartz (T. speciosum, Trill.). Hab. : Ireland, Wrales (established itself in Carmarthenshire, Baker), Spain. In Ireland, 
Trichomanes-continued.

chief locality Turk waterfall, near Killarney, and abundant on Turk mountain, ascending to an altitude of 1,500 feet. Hook. Brit. Ferns, t. 42 .

\section{Davallia.}

D. Canariensis, Smith. Hab. : Spain, Portugal.

\section{Cystopteris.}

C. fragilis, Bernh. Ilab. : rocks and walls chiefly in northern or Alpine regions throughout Europe; Iceland. Hook. Brit. Ferus, t. 23 .

C. alpina, Desv. Hab. : mountains of Europe from Sweden to Greece and Spain, and the Alps. In England, naturalised on a wall at Low Leyton, Es:ex. Hook. Brit. Ferns, t. 24.

C. Sudetica, A. Br. S.Milde. Hab. : Silesia, Sudete mountains of MIoravia, and the C'arpathians.

C. montana, Liml. Hab. : mountains of Scandinavia, and central Europe. In Scotland, rare; in the Breadalbane and Clovi mountains. Hook. Brit. Feris, t. 站.

\section{Adiantum.}

A. Capillus-reneris, Lim. Hab.: throughout the warm parts of Europe. In the south of France growing in the greatest profusion, and luxuriating in the moist perpenclicular sides of the wells. From Jura and Tyrol to Greece, Sicily, and Portugal. 'In Britain, all its certuinly knoun localities, in Comwall, Devon, Wales, and Ireland, are on the coast, and near the level of the sea.' C. Bunbrory; Hook. Brit. Ferns, t. 41.

A. Iithiopicum, Lims. Hab.: Spain.

\section{Cheilanthes.}

('h. frayrans, Trebb \& Berth. Fab. : rocky places throughout the region of the Mediterramean. Switzerland is perhaps its most northern locality. South of France, Sirain, Italy, Greece, Turkey.

Ch. Hispanica, Mettu. Hab. : rochs on the banks of the Mondego, near Loimbra, Portural; Spain.

Ch. Szovitzii, Fisch \& $M$ ('y $\left.^{\prime}\right)$. Hab. : Italy and Dalmatia.

\section{Cryptogramme.}

C. crispa, Br. Hab. : general throughout niddle and northern Europe, especially in mountain regions and moist district;, as far north as Lapland. Siberia, Pyrenees, Spain, Sierra Nerada: eleration, 8-9,000 feet. Frequent on the mountains of Seotland up to ") 3,000 feet of elevation. On rocks and stone walls amoug the English lakes. In Ireland confined to the mountainous countries of the north. Hook. Brit. Ferns, t. :5) and Hook. \& Gr. Ic. Fil. t. :y and 158

\section{Pteris.}

Pt. longifolia, $L$. Hab. : Spain, Dalmatia, Sicily.

Pt. cretica, L. Hab. : south of Switzerland, Italy, Crete, Corsica.

Pt. arguta, Aiton. IIab.: l'ortugal, Serra de Cintra; rare.

Pt. aquilina, $L$. IJab.: abundant in Europe. In Lapland it just passes within the arctic circl ascending in Scotland to 2,000 feet, and there attaining a height of $5-6$ feet in sheltered situations. Hook. Brit. Ferns, t. 38. 


\section{Iomaria.}

L. Spicant, Desv. (Blechnum boreale, Sw.). Hab. : throughout Europe, from Norwegian Lapland to Spain, and the islands of the Mediterranean. Crete. Abundant in England, Scotland, and Ireland. Hook. Brit. Ferns, t. 40.

\section{woodwardia.}

W. radicans, Smith. Hab.: Spain, Portugal, Italy, Ischia, Sorrento, Sicily.

\section{Asplenium.}

A. Hemionitis, Linn. Hab. : Spain, Portugal, or South-Western Europe.

A. viride, Huds. Hab.: Arctic Europe to the Pyrenees, Dalmatia, chiefly in mountain or subalpine regions. 'Most frequently, and growing most vigrorously on calcareous rocks, but not confined to them.'-C. Bunbury. Trondheim, in Norway. In mountain districts of England, Wales, and Ireland; especially abundant in Scotland. Hook, Brit. Ferns, t. 30.

A. Henffleri, Reicharit. Itab. . Tyrol.

A. Trichomanes, $L$. Hab. : temperateregions of the Old World, on stone walls and rocks. Throughout Europe. A rery frequent inhabitant of Great Britain, from the south of Devon and Cornwall to the extreme north in the Orkneys, usually growing from the crevices of walls and rocks. Hook. Brit. Ferns, t. 29.

A. Petrarchre, De ('and. Hab. : Europe; rare. South of France, Montpellier; Vaucluse; Nice, near Toulon; Sicily ; Mount Catalfano, near Bagheria. Clefts of calcareous rocks of Sierra Vernisa, near San Felipe de Xativa, Spain. Hook. \& Gr. Ic. Fil. t. 152.

A. septentrionale, Hoffm. Hab. : Norway ; Britain, to Spain, Italy, and the Ural Nountains. In Britain, in clefts of rocks and walls in the mountain districts. Not known as a native of Ireland. Mook. Brit. Ferns, t. 20;.

A. Seelosii, Leybold. Hab. : Tyrol and Carinthia. Clefts of dolomitic rocks, at an elevation of from 4--6,000 feet, on the south and north-west side of the Schleerngebirg, in South Tyrol. In clefts of calcareous rocks at the foot of Nuunt Geier, near Salum, South Tyrol; perhaps the rarest of all European ferns. Hook. Second C'ent. Ferns, t. 8:).

A. marinum, Limn. Hab. : coast of West Europe, from the Orkneys to the Canaries and Azores; although so common on the roclry coasts of the British Isles (far more general on the west than on the east coasts, and in Ireland, but rarely seen inland), as far north as the Orkneys, it is nowhere known as an inhabitant of Germany or Scandinavia, but following the coasts of France and Spain, it extends south to the Canaries. Hook. Brit. Ferns, t. 31.

A. Germanicum, Triss. Hab. : middle and north of Europe, as far as Stockbolm and Helsingfors; also in Hungary and Dalmatia. 'Much more rare on the Continent than $A$. septentrionale; growing in company with it on stone walls in the valley of Chamounix in Savoy. -C. Bumbury. Rare in England and Scotland; mostly in stony and mountain districts. In Wales, on Helvellyn; Lowlands of Scotland; Minto Crags, near Dunkeld. Hook. Brit. Ferns, t. 27.

A. Ruta-muraria, Lim. Hab. : Arctic Europe to Spain ; common 
Asplenirm-continued.

on rocks and old walls and buildings throughout Great Britain and Ireland. Hook. Brit. Ferns, t. 28.

A. fissum, Fït. Hab.: Southern Gernany and Italy, Hungary, Croatia, Dalmatia, Turkey, Naples, Carniola, Isle of Gothland.

A. Adiantum-nigrum, Lim. Hab. : throughout Europe; Greece, Norway, Britain. Hook. Brit. Ferns, t. 33.

A. fontanum, Bernh. Hab. : Central Europe ; England to Spain, France, Italy, Germany, Greece. On walls and rocks; very rare in England; a dubious native. Hook. Brit. Ferus, t. 34.

A. Ianceolatum, Huds. Hab. : warm parts of Europe; Greece, Spain, Portugal, islands of the Yediterranean; extending north to the southern parts of Britain ; in the greatest abundance within the influence of the sea atmosphere. Hork. Brit. Ferns, t. 32.

A. (Athyrium) crenatum, Ruprecht. Hab. . Norway, Sweden, Lapland; apparently common.

A. (Athyr.) Filix-foemina, Bernh. Hab.: throughout Europe; abundant in England, Scotland, and Ireland, moist shady places, and damp woods. Hook. Brit. Ferns, t. 35 .

A. (Hemidictyum) Ceterach, Lim. Hab. : Britain and Gothland, to Spain and Greece. 'In Italy (speaking generally) this grow. everywhere on dry rocks, and the Adiantum everywhere on wet rocks. They are the two most common ferns.' C: 'Burbury.

\section{Scolopendrium.}

S. rulgare, Sw. Hab.: shady banks, woods, \&c., throughout Europe, from Scandinavia in the north, to Italy, Greece, and Spain; frequent in England, Scotland, and Ireland. Hook. Brit. Ferns, t. 37. Greece.

S. Hemionitis, Su. Hab. : south of France, Italy, Sicily, Spain,

\section{Aspidium.}

A. (Polystichum) Lonchitis, Shr. Hab.: abundant in the temperate and cooler parts of Europe, chiefly on the elevated mountains in the south; and it appears to have an extensive range generally in the northern hemisphere from Greenland (Disco) in the north, to Switzerland, Spain, Portugal and Italy, Greece (Mount Olympus) in the south. In lofty clefts of mountains, among rocks and loose stones, in the north of England and W'ales; much more abundant in Scotland, especially in the Breadalbane and Clova districts; rare in Ireland. Hnok. Brit. Ferns, t. 9.

A (Polyst) aculeatum, Sw. IInb. . almost every part of the lnown world; ras. Aspid. lobatum, Aspid. angulure. Our typical form is common thronghout the temperate, and var. angulare in the warmer parts of Europe, in Spain and Portugal, Greece, Morea, Calalria. Abundant in Great Britain and Ireland, in slady banks and lanes. ILook. Brit. Ferns, t. 10, 11, 1:,

\section{Nephrodium.}

N. (I_astrea) Thelgpteris, Desi: IIah.: hogogy, marshy ground throughout Europe. Nortray, to spain and Italy. In rarious parts of England and Ireland; rare in Scoutland, where it is said to be peculiar to Forfarshire, in the Bog of liestennet. Ilook. Brit. Ferm, t. 18.

N. (Last.) montauum, Brrker ( . Oreopteris, Desv.). Hab. : Lap- 
Nephrodium-continued.

land, to Spain and Greece. Frequent in exposed situations in hilly and mountainous regions in England, Scotland, and Ireland; yıelding a balsamic fragrance when handled, from the copious glands of the frond. Hook. Brit. Ferns, t. 14.

N. (Last.) Filix-mas, Rich. Hab.: throughout Furope. One of the most common of ferms.

N. (Last.) cristatum, Mich. Hab.: Seandinavin, to S. Italy and Greece. Rare in England; chiefly in Norfolk. Hook. Brit. Ferns, t. 17 .

N. (Last.) rigidum, Desv. Hab.: Spain, Greece, Syria, South Germany, Alps of Switzerland, and Savoy; rare in Britain. Hrok. Brit. Ferns, t. 16.

N. (Last.) spinulosum, Desv. (N. dilatatum, N. remotum, \&c.). Hab. : almost universal throughout Europe from the Mediterranean to Sweden and Norway. Hook. Brit. Ferns, t. 18 to 22.

\section{Polypodium.}

P. (Pheg.) Phegopteris, Linn. Hab. : mountain districts generally throughout Europe, from Iceland to Switzerland and Savoy, Etruria and Greece. In various parts of England, Scotland, and Ireland, chiefly in woods; rare in the warmer counties of the south-erst of England. Hook. Brit. Ferns, t. 3.

P. (Pheg.) Dryopteris, Linn. Hab. : Lapland, to the Pyrenees and North Italy, in mountain districts; frequent in the mountain districts of England and Scotland; rare in Ireland; still more rare in the south and east of Fngland. Hook. Brit. Ferns, t. 4.

P. (Pheg.) alpestre, Hoppe. Hab.: Lapland, Russia, Germany, and on the Alps in the south. Rare in the Highland mountains of Scotland. Hook. Brit. Ferns, t. 6.

P. vulgare, Linn. Hab. : common throughout most of the cold and temperate regions of the globe. In Europe to its extreme south. 'Througbout Great Britain and Ireland, on old banks, walls, rocks, and mossy trunks of trees. Hook. Brit. Ferms, t. 2.

\section{Nothochlæna.}

N. Lanuginosa, Desu. Hab. : Spain, and all the warm regions of the Mediterranean, Greece, Syria, \&c.

N. Marantæ, $R$. Br. Hab.: south of Europe, throughout the Mediterranean region. Syria and Tauria, to Ardèche and Portugal. 'As far north as Botzen in the Tyrol $\left(46^{\circ} 30^{\prime} \mathrm{N}\right.$.) ; but both the Nothochlænas are very local in Europe.' C. Bunbury.

\section{Gymnogramme.}

G. Pozoi, Kunze. Hab. : Province of Biscay, and Sierra Nerada, Sprin. G. rutafolia, Hook. \& Gr. Ic. Fil. t. 90; Hook. Cent. Ferns, t. 35 .

G. leptophylla, Desv. Hab. : the south of Europe, Jersey, and shores of the Mediterranean. Hook, \& Gr. Ic. Fil. t. 25.

\section{osmunda.}

O. regalis, Linn. Hab.: Sweden, and most parts of Europe. Bogs and marsby grounds, at the margins of woods, \&c., in rarious parts of England, Scotland, and Ireland. Hook. Brit. Ferns, t. 4.\%

\section{ophioglossum.}

O. lusitanicum, Iimn. Hab. : shores of the Mediterranean, and as 
Ophioglossum-continued.

far north as Guernsey, on summit of rocka, noar Petit Bot BaJ. Hook. Brit. Ferns, t. 47.

O. vulgatum, Lim. Hab. : Lapland and various parts of Europe. In old moist pastures and in woods in Great Britain and Ireland, extending to the extreme north. Hook. Brit. Forrs, t. $46^{\circ}$.

\section{Botrychium.}

B. simplex, Hitch. Hab.: North Europe. (B. Kannenbergii, Klim.s.) Hook. \&. (ir. Ic. Fil. t. . 2 .

B. rutaceum, Sw. Hab. : Scandinavia.

B. Lunaria, Sw. Hab.: Arctic Europe and Iceland, to Spain and Italy. On dry mountain and billy pastures, and downs neal the sea, in various parts of England, Scotland, and Ireland from the extreme south to Orkney and Shetland in the north. Hook. Brit. Ferns, t. $4 \bowtie$.

B. ternatum, Sic. Hab.: Lapland to Pyrenees and Hungary.

B. virginianum, Sw. Hab.: Norway to Austria. Hook. Gard. Ferns, t. 29. (B. Ianuginosum.) Hook. s. Gr. Ic. Fil. t. 79.

\section{ALGERIA, MADEIRA,* CANARIES, AZORES.}

\section{Dicksonia.}

D. Culcita, L'Hérit. Hab. : Mladeira (very rare), and the Azores. In the latter islands, on mountains of 2-3,000 feet of eleration, so abundant that the dense woolly covering of the rhizome has now become an article of commerce.

\section{Hymenophyllum.}

H. Tunbridgense, Snith. IIab.: Madeira; on very wet shaded rocks, by the side of the levada, or aqueduct, between the Ribeiro Frio and Metade valleys; very plentiful, All well-marked examples of $H$. Tunbridgense, not approaching to $H$. Wilsom. In the Canary Islands and Azores, H. unilaterale ( $=\mathrm{H}$. Wilsoni, Hook.). In the islands of Flores and Corro. Hook. Brit. Fons, t. 43.

\section{Trichomanes.}

T. radicans, Swartz (T. speciosum, Tilld.). Hab. : Madeira, in the same locality with the Hymenophyllum; very sparingly. "In Teneriffe, in the greatest profusion in the wood of Agua Carcia, at the elevation of about 2,500 feet.'-C'. Bumbury. In Azores.

* 'Mirdeira is famous for its abundance of Ferns. On the northern slope they grow in surprising profusion. In all the ravines which intersect that coast, we see them clothing the rocks and shady banks, the roots and trunks of trees, the margins of the innmmerable rapid streans, and the moist and mossy walls. Even in the villages they abound. The beautiful Hare's-foot fern, Darallia Canariensis, and the Polypodium vulgare, appeared to me the most generally common ferns throughout the island. 'They are frequent even in the immediate neigbbourhood of Funchiz; and on the northern side of the island, there is hardly a damp wall or an old mussy tree on which they do not flourish. Near the mouth of the St. Vicente river, I saw them growing (in a stunted condition certainiy) among the loose stones of the actual sea-beach. It is on old trees in the mountain woods of Serra d'Anua and Ribeiro Frio that they are mint luxuriant and beautiful; and then they ascend to an elevation of at least 3,000 feet above the sea-level, -Jimrual of Linnaan Society, March 18,in: Remarks on the Botany of Madeira and Teneriffe by Sir Charles Bunbury, Bart. 
Davallia.

D. Canariensis, Smith. IHab. : Madeira; one of the most common ferns; as also in the Canaries. \$\$.

\section{Cystopteris.}

C. fragilis, Bernh. Hab. : Madeira; very abundant on wet and shaded rocks and banks, from very near the level of the sea (on the north coast) to 3,000 feet and upwards in the laurel forests. In the Canaries, in the wood of Agua Mansa, above Orotava, abundant and fine. In Azores, at Fayal, Flores, and Pico. In Algeria.

\section{Adiantum.}

A. reniforme, Linn. Hab : Madeira; under the shade of overhanging rocks, and on rocks constantly wet with the spray of waterfalls and torrents; plentiful in many of the ravines of the north side of the island, mostly at a low or moderate level. At Ribeiro Frio (though very sparingly), and on the precipice immediately below Pico Grande, at an elevation probably exceeding 4,000 feet. Very local on the southern side, though plentiful in some particular ravines, especially in that of Santa Luzia. Also in the Canaries.

A. Capillus-veneris, Linn. Hab.: very common on both sides of Madeira, chiefly in the coast region, but ascending to nearly 2,600 feet in the Santa Juzia ravine. Particularly fine and luxuriant in some of the wet ravines of the north coast. In the Canaries and Azores. In Algeria and Barbary States.

\section{Cheilanthes.}

C. fragrans, Webb \& Berth. (C. odora, Sw.). Hab.: Madeira; on old walls at Funchal, sparingly; sometimes in company with Nothochlæna lanuginosa. Canaries and Algeria.

C. pulchella, Bory. Hab. : Canaries; grows in rather arid, rocky spots, in the wild country (overspread with obsidian) between Icod de los Viños and the village of La Gurncha. \$.

C. Szovitzii, Fisch et Mey. Hab. : Algeria. Kuthn.

\section{Pteris.}

Pt. longifolia, Linn. Hab. : Canaries ; rare and very local; on the margin of a rivulet that crosses the road between Garachico and Icod de los Viños in Teneriffe, at Tazacorte in Palma. In Algeria.

Pt. arguta, Aiton. Hab. Madeira. In the wet shady ravines, very common on the north side of the island, down nearly to the sea-level; extremely abundant and fine at Ribeiro Frio (3,000 feet) and in the woods at the head of the Serra d'Agoa valley. On the south side: in great plenty and luxuriance in the Santa Luzia ravine, in a dwaif state on rocks in the Little Curral. Canaries, in the wood of Agua Manza above Orotova, in Teneriffe. Azores, Fayal, Flores, and Pico.

Pt. Aquilina, Linn. Hab. . Madeira, very common on the island; Canaries, Azores, and Algeria.

\section{Iomaria.}

L. Spicant, Desv. (Blechnum boreale, Sw.). Hab.: Madeira. Common in the mountain woods on both sides of the island, also on shady banks in lanes; more common, and descending to a lower level, on the north side. Canaries, Azores, Fayal, Flores, Pico. 


\section{Woodwardia.}

W. radicans, Smith. Hab. : Madeira; in great plenty on the plateau of Santa Anna, about 1,000 feet (more or less) above the sea, on shady hedge-banks and the margius of streams; very fine at Fibeiro Frio (elevation about 3,000 feet) and in the Metade valley. On the south side: on the margins of streams descending from the Arrebentão; in the Little Curral, below the mill; and in great beauty about the Santa Luzia waterfall. In the Canaries, in the wood of Agua Crarcia, Teneriffe, it grows to extraordinary size and beauty. In the Azores, in the islands of Fayal, Flores, and Pico.

\section{Asplenium.}

A. Hemionitis, Linn. (A. palmatum, Lam.). Hab. : Madeira. Very plentiful along the northern coast, generally below, or not nuch above, 1,000 feet of elevation, descending indeed to a very low level; but also in the mountain woods at the head of the Serra d'Agoa valley, and found at Ribeiro Frio. In the Canaries; in Teneriffe near Garachico, and descending even to the sea-shore. In the Azores, in the Isles of Fayal, Flores, Pico, Algeria.

A. Trichomanes, Limn, (A. anceps, Sol.). Hab.: Madeira. Very general in the island, from the rocls of the coast region (between Funchal and Camara dos Lobos) to the laureI forests, at 3,000 feet and eren higher. Canaries, Azores, Fayal, Flores, A]geria.

A. Petrarchæ, D.C. Hab. : Algiers, at high elevations; rare.

A. monanthemum, Limm. Hab.: Madeira; particularly abundant and tine at Ribeiro Frio (about 3,000 feet). In some lanes near $\mathrm{S}$. Jorge and Santa Anna, perhaps below 1,000 feet. On the south side of the mountains; in company with Woodwardia radicans, and Gymnogramme Lowei; on the shaded margins of small streams below the Arrebentão Peak. Canaries, Azores, Fayal, Flores.

A. marinum, Lim. Hab.: Madeira ; frequent on the north coast, near the mouth of the St. Vicente River (some specimens gathered here as much as two feet high). It has been found on the south const, at Camara dos Lobos and Santa Cruz. Canaries, Azores; at Fayal, Flores, Tangiers.

A. Adiantum-nigrum, Lim. (A. acutum, Bory.). Hab.: Indeira; one of the most common ferns, from the coast up to at least 4,000 feet of elevation. In the neighbourhood of Funchal it is the most common of all. Canaries, Azores; in Tayal, Flores, Algeria.

A. Ruta-muraria, Linn. Hab. : Algeria.

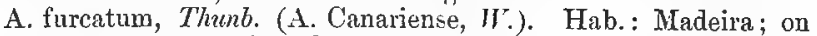
moist mossy stone walls and rocks, on the north side of the island, in many places. Punta Delgada, Bou Tentura, Arco de S. Jorge, and in the ravines that run down to Fayal; most commonly at a moderate elevation, like A. palmatum. Canaries.

A. Lanceolatum, Huds. Hah.: Madeira; on walls near N. S. do Nonte, and in one or two other places not far from Funchal. Azores, in Fayal and Flores, Algeria.

A. (Athyrium) Filix-fœemina, Bernh. Hab. : Madeira ; frequent in moist and shady places. The Canaries, Azores, in Fayal and Flores, Algeria.

A. (Athyr.) umbrosum, J. Sin. (Allantodia umbrosa, R. Br.). Hab. : Madeira; in very wet and shady ravines: Ribeiro Frio, $\mathrm{Me}-$ 
Asplenium-continued.

tade valley, and near the Santa Luzia waterfall. Canaries and Azores.

A. (Hemidictyum) Ceterach, Linn. Hab.: Madeira ; on walls here and there in the neighbourhood of Funchal; occurs sparingly. Canaries and Azores.

\section{Scolopendrium.}

S. vulgare, Sm. Hab. : Madeira; but seemingly rare. It grows in the Santa Luzia ravine, sparingly and small. Azores, in Fayal and Flores, Algeria.

\section{Aspidium.}

A. (Polystichum) falcinellum, Suz. Hab. : Madeira ; in the chestnut woods at Jardim da Serra, and in a more dwarfish state amidst the crowning rocks of the Arrebentâo Peak, at the elevation of more than 3,800 feet. $\$ 8$.

A. (Polyst), aculeatum, Sw. (A. angulare, Sm.). Hab. : Madeira; very common in the mountain woods; also in lanes and hedgebanks on the north side of the island. Canaries, Azores, in Fayal and Flores, Algeria.

A. (Polyst.) frondosum, Lonve. Hab. : Madeira; rocky places, R. de St. Jorge; elevation, 3,500 feet.

\section{Nephrodium.}

N. (Lastrea) Filix-mas, Rich. Hab. Madeira; here and there in the chestnut woods, at 2,000 feet and upwards, but sparingly. (Nephrodium affine, Lowe, does not appear distinct from N. Filixmas.) Canaries, Azores, Algeria.

N. (Last.) spinulosum, Desv. Hab. : Madeira ; common in moist shady places in the mountain woods; particularly fine by the side of the levada, near Ribeiro Frio. (Nephrodium elongatum, Lowe.) Azores.

N. (Last.) æmulum, Baker (N.Fcenisecii, Lowe). Hab. : Madeira ; very plentiful in the laurel forests of the Metade valley and Ribeiro Frio; also above the forests, amidst the bushes of Taccinium padifolium, sin., quite up to the Poizo Pass; nearly 4,600 feet above the sea. Azores, in Fayal, Flores, and Pico.

N. (Last.) unitum, $R$. $B r$. (now Sieb.). Hab. . Algeria.

N. molle, Desv. Hab. : Madeira; on wet banks and under dripping rocks, in the neighbourhood of Funchal ; mostly, if not exclusively, below 1,000 feet of elevation. Azores, in Fayal, Flores, Algeria.

\section{Polypodium.}

P. (Phegopteris) drepanum, Hook. Hab.: Madeira, Rio de St. Jorge. Alt., 3,000 feet.

P. vulgare, Limn. Hab. . Madeira, Canaries; most abundant in Teneriffe, Azores, in Fayal and Flores, Algeria.

\section{Nothocblaena.}

N. Lanuginosa, Desv. Hab.: Madeira, in the crevices of old walls here and there in the city of Funchal. Teneriffe, Algeria.

N. Marastæ, $R$. Br. Hab. : Madeira; very scarce; Canaries, in Teneriffe; abundant, in the coast region, up to about 1,000 feet of elevation.-C. Bunbury. Azores, Barbary States.

\section{Gymnogramme.}

G. (Leptogramme) Totta, Schlecht (G. Lowei, Hook.). Hab. : 
Gymnogramme-continued.

Madeira; on wet rocks near the waterfall of Santa Luzia ; elevation, about 2,600 feet. On the margin of a small stream on the Arrebentão, a little below the summit, the elevation of which is 3,844 feet; also at Ribeiro Frio. Azores, St. Michael's. Hook. \& Gr. Ic. Fil. t. 138.

G. leptophylla, Desv. Hab.: Madeira; in the crevices of walls near the church of St. Antonio, on the south side of the island. Canaries; in the wood of Agua Mansa, athove Orotava. Azores, in Fayal. Algeria. Ilook. \& Gr. Ic. Fil. t. 25.

\section{Acrostichum.}

A. Squamosum, Sw. Hab.: Madeira; at Ribeiro Frio; very sparingly, at the root of decayed trees, by the levada, or aqueduct. Azores; abundant in Fayal, Filores, and Pico.

\section{osmunda.}

O. regalis, Lim. Hab.: Azores, in Fayal and Flores. Algeria. Ophioglossum.

O. lusitaricum, Limn. Hab. : Madeira, Azores.

O. vulgatum, Linn. Hab. . Azores, in Flores. 


\section{ASIA.}

1. NORTHERN, CENTRAL, AND WESTERN ASIA, INCLUDING CHINA AND JAPAN.

2. NORTHERN INDIA, INCLUDING ASSAM, AND ALL NORTII OF THE DECCAN.

3. SOUTHERN INDIA, INCLUDING CONCAN, DECCAN, ORISSA, AND CEYLON.

4. EASTERN PENINSULA AND ARCHIPELAGO, PHILIPPINE ISLES.

$$
\$ \text { denotcs the only hitherto known localities. }
$$

\section{NORTHERN, CENTRAL, AND WESTERN ASIA, INCLUDING CHINA AND JAPAN.}

\section{deichenia.}

G. longissima, $B l$. ( $($ x. excelsa, $J$, Sm.). Hab.: South China, Fokien, Hongkong; Japan, Tsus-Sima.

G. dichotoma, Willd. Hab. : China, Chusan, Honglong; Japan, Nangasaki, Fizen, ins. Kiusiu. Miquel.

\section{Cyathea.}

C. spinulosa, Wrall. Hab. : Ousima, South Japan.

\section{Alsophila.}

A. tomentosa, Hook. Hab.: Formosa.

A. podophylla, Hook. Hab. : Chusan, Hongkong; frequent at the foot of Victoria Peak, in a ravine. Hook. Second Cent. Ferns, t. 66.

A. glabra, Hook. (A. gigantea, $W_{\text {cell. }}$ ). Hab.: China.

A. latebrosa, Hook. Hab. : Formosa.

A. subglandulosa, Hance. Hab. Formosa. \$.

\section{Onoclea.}

O. orientalis, Hook. Hab. : Hakodadi, Japan.

\section{woodsia.}

W. Ilvensis, $R . B r$. Hab. : Japan, Ochotsk, and Siberia. Miquel.

W. polystichioides, Eat. Hab.: Japan, Hakodadi, and Manchuria; Talien-kwan, Yellow Sea, China. Hook. Second Cent. Ferns, t. 2; and Hook. Gard. Ferns, t. $32 . \$$.

W. Manchuriensis, Hook, Hab.: Manchuria. Hook. Second Cent. Ferns, t. 98. \$. 


\section{Dicksonia.}

D. (Cibotium) Barometz, Link. (C. Assamicum, Hook). Hab. . Hongkoug, common in ravines. Bentham.

\section{Hymenop hyllum.}

IL. barbatum, $T^{r} . D . B$. Ilal. : Tsus-Sima, near Japan. $\$$.

H. Japonicum, Miquel. Hab. . Japan. \$.

\section{Trichomanes.}

T. parvulum, Poiret. Hab. : Japan, China, Honglrong.

T. intramarginale, Hook. \& Gr. Hab.: Hongkoug. Benthan; Hook. \&. Gi. Ic. Fil. t. 211 .

'T. latemarginale, Eaton. Hab. . Hongkong. \$.

T. Filicula, Bory. Hab. : Japan, Bonin.

T. auriculatum, Blume. Hab. : Japan, Formosa.

T. rigidum, Swortz (T. strictum, Hook. oै Gr. Ic. Fil. t. 12?). Hab. : Japan and S. China.

\section{Davallia.}

D. pedata, Swith. Hab.: Hongkong, on a rock at the top of Victoria Peak. Hook. Giud. Ferns, t. 7.

D. elegans, swartz. Hab.: China, Canton, Honglkong.

D. divaricata, Blume. Hab.: Hongkong.

D. Griffithiana, Hook. Hab. . China, Amoy, Chusan, Formosa, Hongkong.

J. bullata, Houll. Hab. Japan, Niphon, Nangasaki.

D. Hookeriaua, Wrall. Hab. : Hongkong, in the path to PokeFallum. Bentham.

D. Wilfordii, Baker (D. rhomboidea, Hook. non ITall.). Hab. : Hakodadi, Japan, Jokohama. Hook. Second Cent. Ferns, t. 4s. \$.

D. pilosella, Hool. Hab. : Japan and Tsus-Sima, island oft the coast of Corea. Hook. Serund ('ent. Ferns, t. 96.

D. villosa, Wall. Hab. : China, Japan, Nangasaki, Simodi, Norno Sahi, in Kiusiu. Migrel.

D. strigosa, Swartz. (D. Khasiana, Hook.). Hab. : South-East China, Japan.

D. Speluncæ, Baker (D. polypodioides, Hook.). Hab.: SouthEast C'hina; Hongrong, common in ravines; Japan.

D. tenuifolia, Swartz (D. Chinensis, Swartz). Hab.: China; Hongkong, abundant in the island; Japon, Niphon, Jolrohama, Kiusiu.

\section{Cystopteris.}

C. fragilis, Bernh. Hab. : Kamtschatka.

C. montana, Link. Hab.: Kamtschatka.

\section{Iindsaya.}

L. cultrata, Swartz. Hab.: Japan, Nangasaki.

L. Habellulata, Iry. IIab. : South-East China; Hongkong, in ravines and in the Happy Valley woods. Bentham; IIook. \& Gr, Ic. Fil. t. 75 .

L. ensifolia, Swartz. Hab.: Hongkong, in rarines, and in the Happy Valley woods. Bentham; Hook. Gard. Fins, t. 62.

L. heterophylla, Dry. Fab.: Isle of Loo-Choo. IIongrong, in woods and shady places. Bentham. 


\section{Adantum.}

A. lunulatum, Burm. Hab. : Hongkong. IIook. Gr. Ic. Fil.t. 104.

A. Cantoniense, Hance. Hab. : ramparts of Canton. \$.

A. caudatum, Linn. (A. Edgeworthii, Hook.). Hab.: Hongkong, in moist situations about Little Hongkong.-Bentham. Chusan, Japan.

A. diaphanum, Blume. Hah.: South-East China.

A. capillus-veneris, Linn. Hab. : South-East China and Japan. Ural, Caucasus, Persia, Syria.

A. monochlamys, Eaton. Hab.: Japan, Niphon, Jokohama, TsusSima. Hook. Second Cent. Ferns, t. 50 . \$\$.

A. pedatum, Linn. Hab.: Japan, Manchuria, in Amur, Unalaschka.

A. flabellulatum, Linn. Hab.: very common in various parts of China, Hook. Kew Gardens Misc. v. 9. Hongkong, common in the island.

\section{Cheilanthes.} Japan.

Ch. Mysurensis, Wall. (Ch. Chusana, Hook.). Hab. : cliffs ; Chusau,

Ch. fragrans, Trebb \& Berth. (non Swartz; C. odora and suaveolena, Swartz). Hab. : Afghanistan, Syria.

Ch. varians, Hook. Hab.: South China.

C'b. tenuifolia, Swartz. Hab. : South-East China; Hongkong, in ravines.

Ch. Szovitzii, Fisch \& Meyer. Hab. : Asia Minor and Persia. Rocks near the fort of Schuscha in Talysch, province of Karabagh, Caucasian Alps. Crevices of rocks in ifghanistan.

Ch. argentea, Hook. IIab.: Siberia, in fissures of rocks, near the river Katunja, Altai, Kamtschatka, Dahuria, and Lake Baikal. Japan, China.

\section{onychium.}

O. melanolepis, Decaisne. Hab.: South Persia, between Shiraz and Kazeroum, Island of Karek, Persian Gulf. Hook. Second Cent. Ferns, t. 2.

o. Japonicum, Kunze. IIab. : Japan, in Kosido, Satsuma, Nangasali in Kiusiu, and elsewhere in mountain districts. China.

\section{Pellaea.}

Pteris.

I’. geranirfolia, Fée. Hab. : North China.

Pt. Iongifolia, Limn. Hab. : China, Chusan, Loo-choo, Hongkong, on dry banks about Victoria. Bentham.

Pt. Cretica, Lim. Hab.: Loo-chon, Japan, Jokohama.

Pt. serrulata, Limn. fil. Hab. : China, Hongkong, Japan, Owari, and in woods of the island Kiusiu, near Nangasati, mountain Kawara, Jama. Miquel.

Pt. crenata, Swartz. Hab.: Chusan, Loo-choo. Hongkong, frequent in ravines.

Pt. semipinnata, Linn. Hab.: South-East China; Hongkong, common in ravines and ditches.-Bentham. Japan, Niphon, Nangasaki. Hook. Gard. Ferns, t. 59

Pt. quadriaurita, Retz. Hab.: South China. Hongkong, common in the island.-Bentham. Japan. 
Pteris-continued.

Pt. longipinnula, Itall. Hab. : Japan.

Pt. aquilina, $L$. Hab.: Hongkong, on the hills, and in ravines about Tictoria.-Benthan. Chusan.

Pt. biaurita, $L$. Hab.: S. China.

\section{Ceratopteris.}

C. thalictroides, Brong. Hab.: tropical regions in quiet waters. Japan, near Nangasalii. Hongkong, in the Happy Valley, at the water's edge. Bentham.

\section{Iomaria.}

L. Spicaut, Desv. Hab. . Kamtschatka, Japan, Hakodadi, Caucasian provinces.

L. euphlebia, Kumze. Hab.: Japan, Nangasaki, and Tsus-Sima. Hook. Second Cent. Ferns, t. $89 . \not 5$.

\section{Blechnum.}

B. orientale, Limn. Hab. : China, abundant in Hongkong.

\section{woodwardia.}

W. orientalis, Swz. Hab.: Japan, Loo-choo, Keelung, Isle of Formosa, Jokohama.

W. Japonica, Sw: Hab. : Japan; Ilongkong, on the hills, and in the Fappy Talley.-Benthrm. Chusan. \$.

W. Harlandii, Hock. Hab. . Hongkong, among long grass and low bushes on the first hill going to Victoria Peak. Benthom; Ilook. Fil. Eat. t. 7 . \$.

\section{Asplenium.}

A. Nidus, L. Hab.: Japan, Owari, Bonin Islands, S. China, Canton, Chusan; Hongkong, in the Happy Valley, behind the Budhist temple. Bentham.

A. Trichomanes, Linn. Hab. : Afghanistan, Persia.

A. septentrionale, Hoffm. Ilab.: Ural, Caucasus, and Altai.

1. Irightii, Eitun. Hab.: Takonosima, Japan. $\$$.

A. hirtum, K'unlf. Hab.: Hongkoug.

A. Hancei, Baker. Hab. : SE. China. \$.

A. macrophyllum, Swz. Hab. : China, Hongkong, Bonin Islands.

A. resectum, Smith. Hab.: Japan.

1. heterocarpum, Wall. Hab. : SE. China.

A. lacinictum, Don. Hab. : Japan.

1. Ruta-muraria, Linn. Hab.: Thibet, Siberia, Japan, Niphon, Owari.

1. sepulchrale, Hook. MSS. Hab. : Japan, C'Lina.

1. Adiantum-nigrum, Linn. Hab. : Siberia, Afghanistan, Persia.

A. Solidum, Krmae. IIab. : Japan.

A. cuneatum, Lam. Hab.: Honglong, in the Happy Valley. Bentham.

A. furcatum, Thunb. Hab.: Island of Tsus-Sina.

A. laserpitiifolium, Lam. Hab. . C'hina, Hongkong, Chusan, Bonin Isles.

A. Blakistoni, Bater: Hab.: Province of Szchuan, IV. China.

A. incisum, Thunb. Hab.: Japan; common, Port IIamilton, Nangasaki, Hakodadi, Simoda, Tsus-Sima, and islands in Corea Strait, Chusan, W. China (A. elegantulum), Hook, Second Cent. Ferns, t. 28. 
Asplenium-continued.

A.Wardii, Hook. Hab.: Tsus-Sima, Hook.Second Cent. Ferns,t. 33.

A. (Darea) davallioides, Hook. Hab.: Japan, Nangasalki, Niphon, Tsus-Sima, Loo-Choo, Formosa, Hongkong. Hook. Second Cert'. Ferns, t. 40.

A. (Uarea) rutæfolium, Kunze. (A. prolongatum, Hook.), Hab. : Tsus-Sima, Strait of Corea.

A. (Athyr.) crenulatum, Fries. Hab.: Siberia, Amur, Manchuria, Japan.

A. (Athyr.) spinulosum, Baker. Hab. : Amur-land, Manchuria, Korea, Japan. \$.

A. (Athyr.) cystopterioides, Hook. IIab.: Loo-Choo Islands, Niphon, Owari. Miquel. \$\$.

A. (Athyr.) thelypteroides, Mich. Hab.: Amur, Chinese Tartary.

A. (Athyr.) macrocarpum, Blume. Hab.: Province of Shantung, N. China.

A. (Athyr.) nigripes, Bhume. Hab.: Japan.

A. (Athyr.) niponicum, Mett. Hab.: China, Japan, Niphon, Owari. $\$$.

A. (Athyr.) Filix-foemina, Bernh. Hab. : Japan, Manchuria, Amur, Kamtschatka.

A. (Athyr.) oxyphyllum, Hook. Hab. : Japan.

A. (Dipl.) lanceum, Thunb. Hah. : Japan, Amakerima Island, Nangasaki, Niphon, Tsus-Sima, Loo-choo Island, Formosa, Hongkong (A. subsinuatum). Hook. \& Gr. Ic. Fit. t. 27.

A. (Dipl.) bantnmense, Baker (A. fraxinifolium, Wall.). Hab.: Hongliong, in ravines. Hook. Second C'cht. Ferns, t. 19.

A. (Dipl.) Wichuræ, Mett. Hab. : Japan, Niphon. \$\$.

A. (Dipl.) sylvaticum, Presl. Hab.: Hongkong, in ravines. Benthan.

A. (Dipl.) japonicum, Thunb. (A. Schkuhrii, Mett.). Hab.: China, Loo-Choo, Hongliong, Formosa, Japan, Harbour Island.

A. (Dipl.) lasiopteris, Mett. Hab. : Canton, Japan.

A. (Dipl.) speciosum, 1Iett. Hab. . Honyliong.

A. (Dipl.) virescens, Mett. Hab. : Japan. \$.

A. (Dipl.) squamigerum, Mett. Hab. : Japan. \$

A. (Dipl.) chinense, Baker. Hab.: China, Shanghai. \$.

A. (Dipl.) latifolium, Don. Hab. : S. China; Hongliong, in ravines.

A. (Aniso) esculentum, Presl. Hab.: Hongrkong, in ravines; Formosa.

A. (Hemidictyum) Ceterach, Linn. Hab. Uralian Siberia, Caucasus, Persia, Afohanistan.

\section{Scolopendrium.}

S. vulgare, Sww. Hab. : Japan, Hakodadi, Niphon, Owari, Caucasus, and Asia Minor.

S. (C'ampt.) Sibiricum, Hook. Hab. : Siberia, rirer Angara, Kamtschatka, Japan, Tsus-Sima. Hook. Second Cent. Fu'ns, t. 35.

\section{Aspidium.}

A. (Polyst.) lepidocaulon, Hook. Hab. . Japan, near Simoda; Nangasalki, Tsus-Sima. \$\$.

A. (Polyst.) aculeatum, Swz. Hab.: Bonin Islands, Tsus-Sima, Simoda, Japan. 
Aspidium-continued.

A. (Polyst.) amabile, Blume. Hab.: Formosa, Japan, Niphon.

A. (Polyst.) tripteron, Km dadi, near Simoda, by the side of shady hills. Miquel.; Hook. Second Cent. Ferns, t. $57 . \$$

A. (Polyst.) laserpitifolium, Mett. Hab. : Japan, by the cataracts of Takotsi, \&c. \$.

A. (Polyst.) varium, Sic\%. (A. opaca, Hook.). Ilab.: China, Chusan and Tanglan; Hongkong, in the Tank Ravine, Loo-Choo Islands, Japan, Kiusiu, Hakodadi, Nangasaki. \&.

A. (Polyst.) aristatum, Sic Hah.: China; Hongkong, among rocks in ravines; Tsus-Sima, Japan, Nangasak, Tukure-Sima.

A. (Cyst.) falcatum, Sw . Hab. : Japan; mountains of Nangasaki, Amahirima, Niphon, Jokohama; China and adjacent islands. Loo-Choo, Bouiu; Hongkong, near Tytam Bay. Bentham; Hook. Fil. Exot. t. 92.

A. membranaceum, Hook. Hab. : China, Formosa.

\section{Nephrodium.}

N. decursivo-pinnatum, Baker. Hab.: Japan, Port Chusan, Korea, Formosa. Hook. Second Cent. Ferms, t. 49.

N. (Lastrea) decipiens, Hook. Hab. . China. s.

N. (Last.) podoplayllum, Hook. Hab.: China, Foochow-foo, and Chusan, Hongkong; common in ravines. $\boldsymbol{S}$.

V. (Last.) Sieboldii, Hook. Hab. Japan. Hook, Fil. Exot. t. 31 . $\$$

N. (Last.) gracilescens, Hook. Hab. : Japan, China, Hongkong; on the banks of a ditch at Pockfoolan. Bentham.

r. (Last.) patens, Desv. Hab. : Japan.

․ (Last.) calcaratum, Hook. (N. falcilobum, Hook.). Hab. : Hongliong.

N. (Last.) Thelypteris, $D$.sv. Hab. . Amur-land; Manchuria.

N. (Last.) Filix-mas, Rich. Hab.: China, Hongkong, TsusSima, Hakodadi, Japan, Mauchuria, Port Hamilton.

N. (Last.) lacerum, Batier. Hab.: Japan and Tsus-Sima. \$.

N. (Last.) erythrosum, Hook. Hab. . Simoda, Japan, and TsusSima. \$.

N. (Last.) viridescens, Buker. Hab.: Japan. \$

N. (Last.) fragrans, Rich. Hab.: high-arctic and sub-aretic regions. Caucasus to Kamtschatka, Nanchuria, and Amur.

N. (Last.) Eatoni, Baker. Hab.: Kakeah and Loo-Choo Isles, E. Asia. \$s.

N. (Last.) Chinense, Baker. Hab. : N. China, Korea, Japan. \$. China.

N. (Last.) odoratum, Batier. (N. eriocarpum, Desv.). Hab. : $\mathbf{S}$.

N. (Last.) intermedium, Baker. Thab. : Japnn.

N. (Last.) lepigerum, Baker. Hab. : Isle of Bonin. \$.

N. (Last.) setigerum, Balirr. (Polypodiuni tenericaule, Trall.). Hab.: Tapan, China; Iongkong, near the church of Tictoria, in Tank Ravine and Washerman's Ravine. Bentham.

N. unitum, R. Br. (non Sieb.; N. propinqumw, $B r$.). Hab.: China, Hongliong.

N. Sophoroicles, Desv. ( $($. molle, in part). ITab.: IIonglrong, abundant. Formosa, Japan, Kiusiu, Boniu. \$.

N. pteroides, J. Sm. Hab.: S. China. 
Nephrodium-continued.

N. latipinna, Hook. MSS. Hab. : Hongkong.

N. molle, Desv. Hab. : Honglong; common in ravines.

N. heterocarpon, Moore. Hab. : Hongkong.

N. (Pleo.) Lenzeanum, Hook. Hab. : China, Hungkong.

N. (Sagen.) subtriphyllum, Baker. Hab. : China, Camoens Cave, and elsewhere; frequent, Hongkong.

N. (Sagen.) variolosum, Baker. Hab.: Hongkong; in ravines on rocks in the Happy Valley. Bentham.

N. (Sagen.) decurrens, Baker (Aspid. pteropus, Kze.). Hab. : Formosa, Loo-Choo Islands.

\section{sephrolepis.}

N. cordifolia, Baker (N. tuberosa, Presl.). Hab. : Japan, Niphon, Nangasaki. Miquel.

N. exaltata, Schott. Hab. : Chusan; Hongkong, in ravines.

N. acuta, Presl. Hab. : Lno-Choo ; Hongkong, among rocks on the hill-side near the Happy Valley. Bentham.

\section{Oleandra.}

O. Cumingii, J. Sm. Hab. . Canton.

\section{Polypodium.}

P. (Pheg.) Phegopteris, L. Hab. : Altai, Siberia, Kamtschatka, Manchuria, Japan. Japan.

P. (Pheg.) Dryopteris, L. Hab.: Siberia, Manchuria, Unalaschka,

P. (Pheg.) Oldhami, Baker. Hab. : Formosa. \$. Japan.

P. (Pheg.) punctatum, Thunb. (P. rugulosum, Labill.). Hab.:

P. (Goniopteris) urophyllum, Wall. (Polypodium granulosum, Bentham). Hab.: China, Chusan; Hongkong, in water ravine. Bentham.

P. (Goniopt.) proliferum, Presl. Hab. : S. China.

P. vulgare, L. Hab. : Siberia, Amur, Manchuria, Japan, Erzeroun, Asia Minor.

P. venulosum, Blume. Hab. : Japan.

P. (Goniophlebium) niponicum, Mett. Hab. . Japan; a rare species, found probably in Niphon. Miquel. \$.

P. (Niphobolus.) adnascens, Swz. Hab.: Chusan; Hongkong, common in ravines.

P. (Niph.) Lingua, Swz. Hab. : Japan, Nangasaki, Tsus-Sima. Apparently common in China, Loo-Choo, Formosa, Hongkong.

P. (Niph.) tricuspe, Swz. Hab. : Japan, Fusi and Falcon, Kanagawa, Hakodadi ; south part of Korea ; Port Hamilton, an island on the coast of Korea; alt. 700 feet.

P. (Phymatodes) lineare, Thunb. (P. loriforme, Wall.). Hab.: China, Hongkong, Japan; in craggy places in mountains about Nangasalii, near Simoda, Jokohama. Miquel.

P. (Phym.) superficiale, Bhume. (P. bymenodes, Wall.). Hab.: Hongkong, on rocks in a ravine; Tsus-Sima.

P. (Phym.) linearifolium, Hook. Hab.. Island of Tsus-Sima, Peel Island, Gulf of Korea; growing on rocks along with Polypodium sesquipedale.-Wall. Formosa. Houk. Second Cent. Ferns, t. $58 . \$$. 
Polypodium-continued.

P. (Phym.) normale, Don. Hab.: S. China, Formosa.

P. (Phym.) irioides, Lam. Hab.: China, Macao, Chusan. Hook. Fil. E.cot. t. 4 .

P. (Phym.) ensatum, Thunb. Hab.: Japan; in woody hills near Susolratogi ; in Niphon, Nangasaki, Jokohama, Tsus-Sima. \$.

P. (Phym.) hastatum, Thunb. Hab. : Japan; near the town of Mara, in the island of Niphon; near Nangasaki, Kiusiu, and Simoda (Miquel.), and Formosa. \$.

P. (Phym.) pteropus, Blume. (P. tridactylon, Wall.). Hab.: Formosa, Hongkong; in a wet shady situation in Little Hongkong. Bentham.

P. (Phym.) phymatodes, Linn. Hab. . Hongkong, Formosa, LooChoo, Tsus-Sima.

P. (Phym.) longissimum, Blume. Hab.: Formosa.

P. (Drynaria) conjugatum, Lam. (P. coronans, Wall:). Hab. : Formosa; Honglrong, near Victoria Peak. Bentham.

P. (11y.) Fortunei, Kze, in Metten. Hab.: S. China, both the coast and interior; Foochow; covering the walls of the old fort at Lung-lau, and at Amoy. Province of Szchnon, W. China. \$.

P. (Dry.) quercifolium, Linn. Hab.: S. China.

\section{Nothochlaena.}

N. hirsuta, Desv. (N. sulcata, Link. Hort. Berol.). Hab. : Hongkong, Whampoa.

\section{Gymnogrammo.}

G. (Leptogramme) Totta, Schlecht. (G. Lowei, Hook. \& Gr.). Hab.: Hongkung, Japan, Simoda, Port Hamilton, Korea, Teus-Sima. Hook. \& (ir. Ic. Fil. t. 89.

G. (Lept.) decurrenti-alata, Hook. Hab.: Japan, moist banks; Okosima. $\$$.

G. vestita, Hook. Hab.: Pelin.

G. Javanica, Blume. Hab. . Japan, Simoda, Hakodadi.

G. leptophylla, Desv. Hab.: Karek, in the Gulf of Persia.

G. (Selliquea) lanceolata, Hook. Hab.: China, Bonin, TsusSima, Japan, Nangasaliz.

G. (Sell.) Wrightii, Hook. Hab. : Loo-Choo, Formosa. \&.

G. (Sell.) elliptica, Baker (G. decurrens, Hook.). Hab. : China, Loo-Choo; Hongkong, abundant in ravines; Formosa, Japan, Nangasaki.

G. (Sell.) japonica, Desv. Hab. : Japan, Jokohama, Formosa. \$.

\section{Brainea.}

B. insignis, Hook. Hab.: Hongkong. Hook. Fil. Exot. t. 38.

\section{Meniscium.}

M. simplex, Hook. Hab.: Chusan, Formosa; Hongkong, common on hill-sides. Hook, Fil. Eiot. t. 83. \$8.

M. triphylium, Swartz. Hab. : S. China, Whampoa.

\section{vittaria.}

V. (Tæniopsis) lineata, Swartz. Hab. . Japan.

\section{Drymoglossum.}

D. carnosum, Honk. Hab. : N. China and Japan; abundant. Port Hamilton, Formosa, Hongrong, Loo-Choo, I Kekeato Island. 


\section{Femionitis,}

H. Griffithii, Hook. ( $f l$.) \& Thoms. Hab. : Formosa. (H. Wilfordii.) Hook, Fil. Exot. t. 93.

\section{Acrostichum.}

A. (Stenochlæna) scandens, $J . S m$. Hab.: China.

A. (Egenolfia) appendiculatum, Willd. Hab. : Hongkong.

A. (Gymnopteris) quercifolium, Retz. Hab.: S. China. Hook. Fil. Exot. t. 80.

A. (Gym.) Harlandii, Hook. Hab.: Hongkong, among rocks near the top of Mount Gough ; also in Formosa.-Bentham. (G. decurrens.) Hoo\%. Fil. Exot. t. $94 . \$$.

A. (Gym.) repandum, Blume. Hab.: China, Foochowfoo, Hongkong, 'Tank Ravine, and Water Ravine. Formosa. Bentham.

A. (Gym.) virens, Wall. Hab. : Formosa. Isles.

A. (Chrysodium) bicuspe, Hook. Hab. : Formosa and Loo-Choo

A. (Chrys.) aureum, L. Hab. . Hongkong at Tytamtook, and Formosa.

A. (Hymenolepis) spicatum, Linn. Hab.: S. China. Hook. Fil. Exot. t. 78.

\section{Osmunda.}

O. Javanica, Bhume. Hab. : Kamtschatka; Hongkong, in ravines. Benthrm.

O. cinnamomea, $L$. Hab. : Japan, Jokohama, Manchuria, Amurland. Hook. Gard. Ferns, t. 45.

O. bipinnata, Hook. (non Linn.). Hab. : Hongkong, Tank Ravine. Not known out of the island. Bentham; Hook. Fil. Exot. t. 9. \$.

O. lancea, Thunb. Hab.: Japan. \$

O. regalis, Linn. (var. biformis). Hab.: Japan, Halodadi, Nangasaki ; Niphon, near Jedo. Hongkong, in ravines.

\section{Iygodium.}

L. dichotomum, Swartz (L. circinnatum, Sw.). Hab. : Chusan; Hongkong, in the Happy Valley; in a ravine on Mount Gough, and plentiful behind the Buddhist Temple. Bentham.

L. scandens, Swartz. Hab. : Hongkong.

L. Japonicum, Swartz. Hab. : Japan, Kiusiu, Jokohama ; Hongkong, in ravines; Loo-Choo.

\section{Angiopteris.}

A. evecta, Hoffm. Hab. : Japan, Bonin Sima, Hongkong. Hook. Fil. Exot. t. 75 .

\section{Ophioglossum.}

O. vulgatum, Linn. Hab. : Japan.

\section{Botrychium.}

B. Lunaria, Swartz. Hab. : Kamtschatka.

B. ternatum, Swartz. Hab. : Japan, Niphon, Nangasaki, Siberia.

B. daucifolium, Wall. Hab.: Japan.

B. virginianum, Swartw. Hab. : I'sus-Sima, Niphon, Jokohama. 


\section{NORTHERN INDIA, INCLUDING ASSAM, AND ALL NORTH OF THE DECCAN.}

\section{Gleichenia.}

G. (Mertensia) longissima, Bl. (G. gigantea, Mall.; G. Bancroftii, Hook.). Hab.: Assam, Khasia, Bhotan, Sikkim, Nepal.

G. (Mert.) dichotoma, Willd. Hab. : Assam, Khasia Hills, Silhet, Nepal, Kumaon.

\section{Cyathea.}

\section{Alsophila.}

C. spinulosa, Trall. Hab. . Thhasia, Sikkim, Nepal.

A. comosa, Wall. Hab.: Khasia, Silhet.

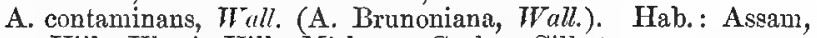
Naga Fills, Khasia Hills, Mishmee, Cachar, Silhet.

A. glabra, Hook. (A. gigantea, $W^{T} a l l$.). Hab. : Silhet, Nepal.

A. latebrosa, Hook. Hab. : Assam; common almost throughout India Proper.

\section{Diacalpe.}

D. aspidioides, $B l$. Hab.: Assam, Silbet.

\section{Onoclea.}

O. orientalis, Hook. Hab.: Silkim Himalaya, elevation, 12,000 feet. Assam. Hook. Second C'ent. Ferns, t. 4 .

\section{Woodsia.}

W. hyperborea, Br. Hab. . Rotang, Himalaya. M. P. Edgeworth.

W. lanosa, Houk. Hab. : Sikkim, alt. 14-16,000 feet; mountains of Kumaon, alt. 11-12,000 feet.

W. elongata, Hook. Hab. : NW. Himalayas, Naglrunda; Hattoo, near Simla; Krande.

\section{Sphaeropteris.} Nepal.

S. barbata, Wall. (Peranema, Don.). Hab.: Khasia, Sikkim,

\section{Dicksonia.}

D. (cibotium) Barometz, Link. (C. glaucescens, and C. Assamicum, Hook. Sp. Fil.). Hab.: Assam.

D. scabra, Wrall. Hab. : Assam, Bhotan, Nepal, Simla; common throughout India.

D. appendiculata, Trall. Hab.: Nepal and Kumaon, Vishnu Ganga. M. P. Fidgeworth.

\section{Fymenophyllum.}

H. exsertum, Wall. Hab.: Himalayas, Nepal.

H. microsorum, $V . D . B$. Hab.: Sikkim-Himalayn; gathered by Dr. Hooker at an elevation of 11,000 feet.

II. polyanthos, Swartz. Hab.: Assam, Sikkim, Nepal.

H. badium, Hook. \& Gr. Hab. : Nepal, Siklim.

H. Javanicum, Spreng. (H. crispatum, Hook. \& Gr. Ic. Fil. t. 77). Ilab. . Assam, Siklim, Nepal; throughout India.

H. Simonsianum, Hook. Hab.: Khasia Hills and Sikkim. Hook. Second Cent. Ferns, t. 13.

H. Smithii, Hook. (H. flaccidum, T. D. B.). Hab. : Khasia. 


\section{Trichomanes.}

T. nanum, $V . D . B$. Hab. : Assam.

T. Filicula, Bory. Hab. : Assam, Nepal, Kumaon, Simla.

T. pyxidiferum, $L$. Hab.: Hindostan as far north as Khasia. Hook. \& Gr. Ic. Fil. t. 206.

T. radicans, Swartz (T. speciosum, Willd.). Hab. : Khasia Hills, mountains of Nepal.

T. auriculatum, $B l$. (T. dissectum, $J$. Sm.). Hab.: Khasia Hills.

\section{Davallia.}

D. pedata, Smith. Hab.: Khasia; Silhet, southward. Hook. Gard. Ferns, t. 7.

D. Assamica, Bedd. Hab. : Assam, Bhotan.

D. membranulosa, Wall. Hab.: Himalayas of Bhotan, Nepal and Kumaon.

D. immersa, Wall. Hab. : Himalayas, ascending to 6-8,000 feet. Kangra, Masuri, Sheopore mountain, Nepal. Paras-Nath. Hook. Fil. Exot. t. 79.

D. multidentata, Hook. MSS. Hab. : Himalayas of Nepal ; Sikkim, 5-7,000 feet, Assam.

D. pulchra, Don. Hab.: Khasia, Himalayas of Nepal, and Sikkim, up to 11-12,000 feet.

D. chærophylla, Irall. Hab.. Assam, Nepal, Masuri, Simla, 9-10,000 feet elevation. Kashmir, Kangra, Kulu. M. P. Edgeworth.

D. nodosa, Hook. Hab.: Assam, Khasia, Bhotan, Sikkim, 7-10,000 feet.

D. repens, Desv. (D. Boryana, Presl.). Hab. . Brahmakund in Upper Assam, and Khasia.

D. divaricata, Blume. (D. polyantha, Hook.). Hab.: Khasia, Nishmee.

D. Griffithianum, Hook. Hab.: Himalayas of Assam, Khasia, and Bhotan.

D. bullata, Wall. Hab. : Assam : Nepal, 3-4,000 feet.

D. Hookeriana, Wall. Hab. : Assam, Khasia, Silhet, Kumaon.

D. villosa, Wall. Hab. : Khasia, Nepal, Kumaon.

D. strigosa, Swartz (D. Khasiyana, Hook.). Hab.. Khasia; Himalayas, 3-5,000 feet.

D. platyphylla, Don. (D. lonchitidea, Wall.). Hab.: Khasia, Bhotan, Nepal. IIook. Fil. Erot. t. 19.

D. urophylla, Hook. Hab.: Khasia, Bhotan, Kumaon.

D. hirta, Kaulf. Hab. : Nepal.

D. Speluncæ, Baker (D. polypodioides, Hook, and D. Jamaicensis, Hook.). Hab. : Assam, Nepal; Himalayas, ascending to 1 7,000 feet, throughout India.

D. tenuifolia, Swartz. Hab. : Assam, Khasia, Silhet, Nepal, Kumaon, Simla; Himalayas, 5,000 feet.

\section{Cystopteris.}

U. fragilis, Bernh. Hab. : Afghanistan; Himalayas, ascending to 15,000 feet. Nepal, Simla, Punjab, Kulu-Kangra. M. P. Edgeworth.

\section{Iindsaya.}

L. cultrata, Swartz. Hab. : Assam, Khasia, Nepal ; Himalayas, to 4,000 feet. Hook. \& Gr. Ic. Fil. t. 144; Hook. Fil. Exot. t. 67. 
Lindsaya-continued.

L. pectinata, Bl. (L. oblongifolia, Reinw.). Hab. : Assam.

L. ensifolia, Swartz. Hab.: Khasia; Himalayas, to 4,000 feet. Hook. \& Gr. Ic. Fil. t. 111; Hook. Gard. Ferns, t. 62.

\section{Adiantum.}

A. lunulatum, Burm. Hab.: Himalayas, 4,000 feet. Assam, Khasia, Silhet, Nepal, Kumaon, Simla, Banda, Calcutta.

A. caudatum, Linn. (A. Edgeworthii, Hook.). Hab. : apparently throughout India. Himalayas, 3,000 feet. Assam, Mishmi, Bhotan, Silhet, Bahar, Nepal, Dacca, Burdwan, Garhwal, Simla, Multan Scinde.

A. Capillus-veneris, Linn. Hab.: Himalayas, up to 6,000 feet. Assam, Khasia, Bhotan, Nepal, Kumaon, Simla, Scinde. In wells, throughout India. M. P. Edgeworth.

A. venustum, Don. Hab.: Assam, Khasia, Nepal ; Himalayas, up to 7-8,000 feet. Meerut, Masuri, Simla. M. P. Edgezvorth.

A. pedatum, $L$. Hab.: Kumaon; Jumnotri, at the source of the Jumna ; Garwhal-Kangra, ascending 7-9,000 feet.

A. flabellulatum, $L$. Hab. Assam, Khasia, Nepal, Kumaon.

\section{Cheilanthes.}

Ch. fragrans, Webb \& Berth. Hab. : NW. Himalayas, at 5,000 feet.

Ch. varians, Hook. Hab.: Assam, Khasia, Silhet, Himalayas, ascending 2-3,000 feet.

Ch. subvillosa, Hook. Hab.: Pabur Valley, and Simla, Himalayas. M. P. Eageworth.

Ch. Dalhousia, Hook. Hab.: Simla, Kumaon; Himalayas, ascending 10,000 feet.

Ch. tenuifolia, Swo. Hab. . Assam, Khasia, Himalayas.

Ch. Szovitzii, Finch \& Meyjer. Hab.: Indus Valley, 7,500 feet, rocks. Scinde, Chahil Tan, Kashmir ; N.-Western Thibet, 8,500 feet.-Dr. T. Thomson, Kulu. M. P. Edyeworth.

Ch. rufa, Don. Hab. : Mishmee, Khasia, Nepal ; Simla, ascending to 4,000 feet.

Ch. argentea, Hook. Hab.: Khala-Panee; Khasia, on wet rocks, alt. 5,500 feet. (Hook. Fil. Exot. t. 95.) Ch. chrysophylla. Hodk. Cent. Ferns, t. 1.

Ch. farinosa, Kaulf. Hab.: Assam, Khasia, Nepal; Almora and Naini Tal in Kunaion, Masuri; Simla, 5,000 feet of elevation. Scinde. Hook. \& Gr. Ic. Fil. t. 134.

\section{onychium.}

O. auratum, Kaulf: Hab. : Himalayas, ascending to 5,000 feet. Nepal, Bhotan, Khasia.

O. Japonicum, Kunze (O. lucidum, Spreng.). Hab.: north of India, ascending in Sikkim to 9-10,000 feet; Khasia, Mishmi, Nepal, Kumaon, Garwhal, Naini Tal, Masuri, Simla.

\section{Cryptogramme.}

C. crispa, $B r$. Hab. : the higher Alps of Kumaon, 12,000 feet; Balti in NW. Thibet; Shayuk valley, elevation 9,000 feet.-Di. Thmmson. Chor Mountain, Simla.-M. P. Edgeworth. Interior of Sikkin-Ilimalaya, 11-13,000 feet. Hooker \& Thomson. 


\section{Pellea.}

P. gracilis, Hook. Hab. : Himalayas of Thibet, 9-10,000 feet. Kumaon, Chamba.

P. Tamburii, Hook. Hab.: Tambur valley, East Nepal. Dr. Hooker.

P. (Allosorus) nitidula, Baker (Cheilanthes, Hook. $S p$. .). Hab. : Himalayas, ascending to 6,000 feet. Kumaon; Simla, on rocks; Pundlkester, Kulu. M. P. Edgeworth; Hook. Cent. Ferns, t. 12.

P. (Platyloma) calomelanos, Link. Hab. : Himalayas, ascending to 6,000 feet; Simla, Kumaon, Karli. II. P. Edgeworth.

\section{Pteris.}

Pt. longifolia, $L$. Hab. . Himalayas, up to 4,000 feet. Naini Tal, Kumaon; universal on the continent of India.

Pt. Cretica, $L$. Hab. : Himalayas, 8-9,000 feet. Khasia, Sikkim-Himalaya, Bhotan, Eastem Nepal, Kumaon, Masuri, Simla, Punjab.

Pt. pellucida, Presl. Hab. : Himalayas, Mishmi, Assam, Cachar, Silhet, Nepal.

Pt. dactylina, Hook. Hab. : dry sheltered rocks, Eastern Nepal, Siklkim-Himalaya, and Khasia, ascending to 9,000 feet.

Pt. crenata, Swz. Hab.: Assam, Khasia, Goalpara, Silhet, and Terrya Ghat, below Dorjiling.

Pt. Griffithii, Hook. Hab. : Assam, Mishmi.

Pt. semipinnata, Linn. Hab. : Himalayas, 3-4,000 feet; Khasia, Silhet. Hook, Gard. Ferns, t. 59.

Pt. quadriaurita, Retz. Hab. . Assam, Gowhatti, Bhotan; Sikkim, 3-11,000 feet. Nepal, Kumaon, Punjab, Rajaori mountains, Scinde.

Pt. longipinnula, Wall. Hab.: Assam; Khasia, 2-3,000 feet; Silhet, Bhotan; Sikzim, 2-4,000 feet; Nepal.

Pt. excelsa, Gaud. Hab.: Nepal, Silhet, Kumaon, Garwhal; Simla, 8,000 feet.

Pt. pellucens, Agardh. Hab.: hills of Assam, Khasia, and Sikkim.

Pt. aquilina, Linn. Hab.: Himalayas, 2-3,000 feet. Khasia, Silhet, Nepal, Kumaon; Garwhal, 7,500-9,000 feet. Sikkim.

Pt. biaurita, Linn. Hab. : Assam, Khasia; Sikkim, 1-4,000 feet. Nepal, Kumaon, Párasnáth. M. P. Edgeworth.

Pt. Wallichiana, Agardh. Hab. : Himalayas, ascending to 8,000 feet. Khasia, Sikkim, Garwhal, Kumaon, Kangra in Kulu.

Pt. incisa, Thunb. (Pt. aurita, Bl.). Hab. : Khasya, Sikkim.

Pt. tripartita, Swz. Hab. : Silhet, Bengal.

\section{Ceratopteris.}

C. thalictroides, Brong. Hab.: quiet waters in tropical and sub-tropical regions. Abundant in the 'Jheels' and other still pools, frequently such as are occasionally dry, in all the warm regions, from the Punjab, Kulu, Rohilkhand, Burdwan. M.P. Edgeworth.

\section{Iomaria.}

L. adnata, Blume. Hab. . Khasia, 1-4,000 feet. Hooker (fil.) \&. Thomson. 
Lomaria-continued.

L. glauca, Blume. Hab, : Khasia Hills, at 6,000 feet. Hooker (fl.) \& Thomson.

L. pyenophylla, Kunze. Hab.: Assam, Khasia, Bhotan, Nepal; Sikkim, 7-10,000 feet. Hooker ( $f(l)$ ) \& Thomson.

L. euphlebia, Kunze. Hab.: Assam, Khasia, Nepal; temperate region, alt. 6,000 feet. Hook. Second Cent. Ferns, t. 89.

\section{Blechnum.}

B. nitidum, Presl. Hał.: Assam, Mishmi.

B. serrulatum, Rich. (B. striatum, Brr). Hab. : Mishmi, in marshes.

B. orientale, Linn. Hab. : Nepal, Silhet, lower hills of Silkim, and Khasia; elevation, 4,500 feet.

B. melanopus, Hook. Hab. : Khasia Hills. Bengal.

\section{woodwardia.}

W. radicans, Smith. Hab. : Himalayas, 4-5,000 feet elevation; Simla; Kangra, in Kulu.

\section{Asplenium.}

A. Nidus, Linn. Hab.: Himalayas. Assam.

A. Grevillei, Wall. Hab. : Mishmi.

A. Simonsianum, Hook. Hab. : Khasia and Unker Hills. Hook. Cent. Ferns, t. 25.

A. ensiforme, Wall. Hab. : Assam, Nepal, and Sikkim; elevation, 7-10,000 feet. Kumaon, 8,000 feet; frequent. Hook. \& Gr. Ic. Fil. t. 71 .

A. Griffithianum, Hook. Hab. : Assam, Mishmi ; Sikkim below Dorjiling, ascending to 4,000 feet. Hook. Cent. Ferns, t. 28.

A. alternans, $W$ all. Hab. : North-west Himalayas, at elevations of not less than 6,000 feet, in stony woods. Kumaon, Simla, Chumbra Hills, Hook. Gard. Ferns, t. 38.

A. viride, Huds. Hab. : Himalaya, Glacier of Pindari ; Kumaon, elevation, 12,000 feet.

A. Trichomanes, Linn. Hab. . Kashmir, Ladak, Masuri, Simla ; Kumaon, 6-12,000 feet elevation; Bhotan.

A. normale, Don. (A. multijugum, Wall.). Hab. . Himalayas of Nepal, Siklim, Assam, Khasia, Silhet.

A. septentrionale, Hoffm. Hab.: Himalayas; Kashmir, elevation, 9,000 feet. Garwhal, 11,000 feet.

A. caudatum, Forst. Hab. : Khasia.

A. macrophyllum, Swartz. Hab. . Assam.

A. resectum, Smith. Hab.: Khasia, elevation, 8,000 feet; Bhotan, Siklkim-Himalaya, Nepal, Simla.

A. heterocarpum, Wall. Hab. : Khasia, Nepal.

A. planicaule, Wall.: Hab.: Khasia, 4-6,000 feet elevation. Sikkim-Himalaya, Kumaon, Garwhal ; Nepal, above Simla.

A. laciniatum, Don. Hab. . Assam, Khasia, Silhet, Bhotan, Duphla Hills, Sikkim-Himalaya, Nepal.

A. Ruta-muraria, Linn. Hab.: Kashmir.

A. Adiantum-nigrum, Linn. Hab. : Kashmir, Simla, Masuri.

A. furcatum, Thunb, Hab. : Assam.

A. nitidum, Swartz. Hab. : Assam, Sikkim.

A. laserpitiifolium, Lam. Hab. : Assam. 
Asplenixm-continued.

A. fontanum, Bernh. Hab. : Himalayas. Beddome.

A. varians, Hook. \& Grev. Hab.: Northern India, frequent, especially on the Himalayas, at elevations $6-8,000$ feet, from Istrardo and Masuri on the west to Bhotan on the east. Lachung river in Sikkim, 9,000 feet. Hook. \& Gr. Ic. Fil. t. 172.

A. bulbiferum, Forst. Hab. : Nepal, Sikkim, Bhotan.

A. tenuifolium, Don. (A. concinnum, Wall.). Hab. : Mishmi, Khasia, Sikkim-Himalayas, Nepal. Hook. Second Cent. Ferns, t. 29.

A. (Darea) rutæfolium, Kze. (A. prolongatum, Hook.). Hab.: Khasia, Bhotan; Mishmi, on trees. Hook. Second Cent. Ferns, t. 42.

A. (Athyr.) subtriangulare, Hook. MSS. Hab.: Sikkim, $10-12,000$ feet.

A. (Athyr.) Hohenackerianum, Kze. Hab.: Scinde.

A. (Athyr.) thelypteroides, Michx. Hab.: Sikkim-Himalaya, elevation, 9-10,000 feet, north-west provinces, above Simla.

A. (Aythr.) macrocarpum, Blume. Hab. : Upper Assam ; Khasia, 5-6,000 feet elevation. Sikkim.

A. (Athyr.) nigripes, Blume. Hab.: Himalayas, ascending to 10,000 feet.

A. (Athyr.) Filix-fomina, Bernh. (A. pectinatum, Wall.). Hab. : Himalayas, 10-12,000 feet, from the extreme north-west. Simla, Bhotan.

A. (Athyr.) oxyphyllum, Hook. Hab. : Himalayas, to 7,000 feet. Assam, Khasia, Bhotan, Nepal.

A. (Athyr.) fimbriatum, Hook. Hab.: Himalayas, ascending 8-9,000 feet. Sikkim, Nepal, Kumaon, Simla. \$\$\$

A. (Athyr.) umbrosum, $J$. Sm. (A. australe, Brack.). Hab.: Himalayas, 6-8,000 feet. Nepal, Sikkim; very frequent.

A. (Diplazium) lanceum, Thunb. (A. subsinuatum, Hook. \& Gr.). Hab. : Himalayas, Nepal, Khasia. Hook. \& Gr. Ic. Ferns, t. 27.

A. (Dipl.) pinnatifido-pinnatum, Hook. Hab. : Mishmi. \$8.

A. (Dipl.) bantamense, Baker (A. fraxinifolium, Wall.). Hab. : Assam, Khasia. Hook. Second Cent. Ferns, t. 19.

A. (Dipl.) longifolium, Don. (A. lobulosum, Wall.). Hab. : Himalayas, Nepal, Simla.

A. (Dipl.) tomentosum, Hook. (non Mett.). Hab.: Khasia.

A. (Dipl.) japonicum, Thunb. (A. Schkuhrii, Mett.). Hab.: Nepal. North-western Himalayas.

A. (Dipl.) Sorzogonense, Presl. Hab. : Himalayas; Sikkim, elevation, 8-10,000 feet. Bhotan.

A. (Dipl.) polypodioides, Mett. Hab. : Himalayas, up to 8,000 feet. Khasia, Bhotan, Sikkim, Kumaon, Simla, Kashmir.

A. (Dipl.) Griffithii, Baker. Hab. : Mishmi, Assam. Hook. Cent. Ferns, t. 27.

A. (Dipl.) maximum, Don. Hab.: Himalaya, Khasia, and Sikkim.

A. (Aniso.) heterophlebinm, Mett.MSS. Hab.: Assam, Mishmi. \$.

A. (Aniso.) decussatum, Swartz. Hab, : Himalayas, Assam.

A. (Aniso.) esculentum, Presl. Hab.: Himalayas.

A. (Hemi.) Ceterach, Linn. Hab. : Himalayas, Kashmir.

A. (Hemi.) Finlaysonianum, Wall. Hlab.: Assam, Khasia, 
Asplenium-continued.

Gowhatti, Mishmi, Nepal, Sikkim, Kamaoun. IIook. Cent. Ferns, t. 37 .

Allantodia.

A. Brunoniana, Wall. Hab.: Himalayas, up to 6,000 feet. Assam, Siklim, Nepal, Sheopore mountain.

\section{Actiniopteris.}

A. radiata, Link. Hab. : Northern India, Scinde, Moradabad; old wall, foot of the limestone hills, Agra. Hook. Cent. Ferns, t. 75 .

\section{Aspidium.}

A. (Polyst.) Lonchitis, Sws. Hab.: Himalayas; on high mountains among birch, at Pye Pundo.

A. (Polyst.) Lachenense, Hook. Hab.: Sikkim-Himalaya, elevation, 13-16,000 feet. Lachen River, Sikkim. \$.

A. (Polyst.) auriculatum, Swz. Hab. : Khasia, Sikkim; Bhotan, elevation, 9,000 feet; Nepal; Kumaon, elevation, 2,600 feet; Naini Tal, Simla, Harabagh.

A. (Polyst.) ilicifolium, Don. Hab. : Sikkim, Changtam, Tambar River (Hook. ( $f i l$.) \& Thomson), Nepal; Kumaon, elevation, 9,000 feet; Simla; Talley of the Jumna, 9,000 feet. $\$$.

A. (Polyst.) Thomsoni, Hook. Hab. : Siklkim-Himalaya ; Kumaon, elevation, 9-13,000 feet above Simla. Hook. Second Cent. Ferns, t, 25. \$.

A. (Polyst.) aculeatum, Swz. (Aspid. rufo-barbatum, Wall.; Aspid. squarrosum, Don.). Hab. : all along the Himalayan range. Assam, Khasia, Silhet, Nepal, Bhotan, Kumaon.

A. (Polyst.) Prescottianum, Hook. Hab.: the whole range of the Himalayas, from the extreme west to Bhotan in the east; elevation, 10-12,000 feet.

A. (Polyst.) amabile, Bhume. Hab. : Nepal.

A. (Polyst.) rristatum, Sws. Hab.: Bhotan, Nepal; Khasia, elevation, 4,500 feet. Hook. ( fil.) \& Thomson.

A. (Polyst.) Sikkimense, Baker. Hab.. Mon-Lepcha; Nepal, $10-11,000$ feet. $\$$.

A. (Polyst.) fœeniculaceum, Hook. Hab.: Chola; Sikkim, 7-10,000 feet. Hook. ( fil.) \& Thomson. \$.

A. (Cyrt.) caducum, Wall. Hab.: north of India; frequent. Assam, Khasia, Sikkim, Nepal, Bhotan. Hook. \& Gr. Ic. Fil. t. 171.

A. (Cyrt.) falcatum, Swoz. (A. caryotideum, $W^{T}$ all.). Hab. . Bhotan, Siklrim, Nepal; Kumaon, elevation, 8,200 feet; Simla. Hook. Gurd. Ferns, t. 13.

\section{Nephrodium.}

N. (Lastrea) cuspidatum, Baker (Polyp. elongatum, Wall.). Hab. : Khasin, Nepal.

N. (Last.) hirtipes, Hook. Hab. : Himalayas, up to 6,000 feet. Nepal; very abundant.

N. (Last.) gracilescens, Hook. Hab. : Khasia, Simla.

N. (Last.) immersum, Hook. Hab. : Assam.

N. (Last.) calcaratum, Hook. (N. falcilobum, Hook.). Hab.: Bengal to Silkkim.

N. (Last.) canum, Baker. Hab. : Himalayas. $\$$. 
Nephrodium-continued.

N. (Last.) prolixum, Baker (N. appendiculatum, Hook.; and N. octhodes, Hook.). Hab.: Assam, Khasia, Nepal, Dorjiling, Sikkim.

N. (Last.) Thelypteris, Desv. Hab.: Kashmir, 5-6,000 feet; and Khasia.

N. (Last.) apiciflorum, Hook. Hab. : Nepal; Sikkim-Himalaya, 9,000 feet. Hook. ( fil.) \& Thomson. \$.

N. (Last.) syrmaticum, Baker (N. spectabile, Hook.). Hab. . Assam, Khasia, Sikkim.

N. (Last.) Filix-mas, Rich. Hab.: Himalayas, Nepal; Sikkim, alt. 8-10,000, and even to 15,000 feet.

N. (Last.) flaccidum, Hook. Hab. : Khasia, Kunawar.

N. (Last.) Brunonianum, Hook. Hab.: Sikkim-Himalaya, 12-15,000 feet; Kumaon, Punjab.

N. (Last.) barbigerum, Hook. Hab.: Kumaon, Simla, SikkimHimalaya, 12-13,000 feet. Hook. ( fll.) \& Thomson. \$\$.

N. (Last.) spinulosum, Desv. Hab. : Western Himalaya.

N. (Last.) sparsum, Don. (N. purpurascens, Hook.). Hab. : throughout the Himalaya range. Assam, Khasia, Nepal.

N. (Last.) Falconeri, Hook. Hab. : Kashmir. \$.

N. (Last.) odoratum, Baker (N. eriocarpum, Dec.). Hab.: Sikkim-Himalaya, Kumaon, Simla.

N. (Last.) membranifolium, Presl. Hab.: Assam, Khasia, Bhotan, Silhet, Chittagong.

N. (Last.) splendens, Hook. Hab.: Sikkim-Himalaya, Bhotan, Nepal.

N. (Last.) angustifrons, Baker. Hab.: Nepal. \$.

N. (Last.) intermedium, Baker. Hab. : Northern India.

N. (Last.) Boryanum, Baker (non Hook.). Hab. . Himalayas, up to 7,000 feet. Assam, Bhotan, Nepal, Khasia, Kumaon, Simla.

N. (Last.) setigerum, Baker (N. tenericaule, Hook.), Hab.: Assam.

N. (Iast.) platypus, Hook. Hab.: Khasia.

N. unitum, $R$. Br. (non Sieb. N. propinquum, Br.). Hab.: Assam, Khasia, Chittagong.

N. pteroides, $J$. S $\mathrm{m}$. Hab. : Himalayas.

N. extensum, Hook. Hab. : Assam, Khasia.

N. cucullatum, Baker (N. unitum, Sieb.). Hab.: Himalayas.

N. hirsutum, $J$. Sm. Hab. : Assam.

N. aridum, Baker. Hab. : Northern India.

N. glandulosum, J. Sm. Hab. : Himalayas, Assam.

N. pennigerum, Hook. Hab. : Himalayas.

N. molle, Desv. Hab.: Himalayas, Chumba, Kumaon, and Banda. M. P. Edgeworth.

N. crinipes, Hook. Hab. : Sikkim-Himalaya.

N. ferox, Moore. Hab. : Kumaon.

N. truncatum, Presl. Hab.: Northern India.

N. (Pleo.) Lenzeanum, Hook. Hab. : Mishmi, Assam, Silhet.

N. (Sagen.) vastum, Baker (Aspid. alatum, Wall.). Hab. . Assam, Khasia, Silhet. Hook. \& Gr. If. Fil. t. 184.

N. (Sagen.) polymorphum, Baker. Hab.: Himalayas, up to 4,000 feet. Assam, Mishmi, Bhotan, Khasia, Sikkim, Kumaon. 
Nephrodium-continued.

N. (Sagen.) variolosum, Baker. Hab.: Parasnath, Telyn above Silhet.

N. (Sagen.) decurrens, Baker (Aspid. pteropus, Kze.). Hab. . Assam and Khasia.

N. (Sagen.) cicutarium, Baker (Aspid., Sw.). Hab.: Himalayas, up to 4,000 feet.

N. (Sagen.) giganteum, Baker (Aspid., Bl.). Hab.: Assam.

\section{INephrolepis.}

N. exaltata, Schott. Hab.: Himalayas, Bhotan.

\section{oleandra.}

O. neriiformis, Cav. Hab.: Assam, Mishmi, Khasia, Sikkim, Nepal. Hook. Fil. Exot. t. 58.

O. Wallichii, Hook. Hab.: north of India, among the mountains from Simla to Kumaon; Assam, Khasia, Bhotan. A most delicate variety is found in the Himalaya, alt. 7,000 feet. \$.

O. Cumingil, J. Sm. Hab. : Assam.

\section{Poly podium.}

P. (Pheg.) erubescens, Wall. Hab. : throughout the Himalayan range, Sikkim, alt. 2,000 feet. Khasia, Kumaon. P. (Pheg.) appendiculatum, Wall. Hab.: East Himalayas,
Nepal.

P. (Pheg.) auriculatum, Wall. Hab.: Himalayas, up to 6,000 feet. Assam, Khasia, Sikkim, Nepal, Simla.

P. (Pheg.) distans, Don. (P. paludosum, Blume.). Hab.: Assam, Khasia ; Siklim, 9-10,000 feet. feet.

P. (Pheg.) Dryopteris, Limn. Hab. : Western Himalaya, 5-8,000

P. (Pheg.) punctatum, Thunb. Hab.: Northern India.

P. (Goniopteris) urophyllum, Wall. Hab.: Assam, Sikkim.

P. (Goniopt.) proliferum, Presl. Hab.: Northern India, apparently universal in hilly regions. Hook. Second Cent. Ferns, t. 15.

P. (Goniopt.) lineatum, Colebr. Hab. : mountains of Northern India, Mishmi, Silhet, Nepal; Kumaon, alt. 3,500 feet; Simla.

P. (Goniopt.) multilineatum, Wall. Hab.: Assam, Gowhatti, Bhotan, Silhet, Sikkim, Parasnath, Pundua; Kumaon, up to 3,000 feet.

P. Khasyanum, Hook. Hab. : on trees, Khasia, alt. 4,000 feet. Hook. Cent. Ferns, t. 49 . \$.

P. trichomanoides, Sivz Hab.: Sikkim-Himalaya.

P. parvulum, Bory. Hab. : N. India, up to 8,500 feet.

P. subfalcatum, Blume. Hab. : Kumaon, alt. 8,500 feet; Siklim-Himalaya. Nepal.

P. dareæforme, Hook. Hab.: Sikkim-Himalaya, Khasia hills. Hook. Second Cent. Ferns, t. 24.

P. subdigitatum, Blume. (P. davallioides, Mett.). Hab. : Nepal. Siklim; 7-8,000 feet. Bhotan.

P. (Goniophlebium) amœnum, Trall. Hab.: Khasia, Nepal, Bhutan, Kumaon, Masuri ; Dalhousie, alt. 6-10,000 feet.

P. (Gonioph.) lachnopus, Wall. Hab.: Khasia, 4-6,000 feet. Siklim, 9-11,000 feet. Nepal, Kumaon, Simla. Hook. Cent. Ferns, t. 52. 
Polypodium-continued.

P. (Gonioph.) persicæfolium, Desv. (P. cuspidatum, Blume.). Hab. : Nepal. Beddome.

P. (Gonioph.) subauriculatum, Blume. (P. argutum, Wall.). Hab.: Himalayas, Khasia, Gowhatti plains ; Sikkim, 8,000 feet. Nepal, Kumaon, Naini Tal.

P. (Niphobolus.) adnascens, Swz. Hab. : Assam, Khasia, Silhet, Sikkim-Himalaya, Nepal, Kumaon. Hook. Gard. Ferns, t. 19.

P. (Niph.) Lingua, Swz. Hab.: Eastern Himalaya; Bhotan, 5,000 feet.

P. (Niph.) stigmosum, Swz. (P. costatum, Wall.). Hab. : -Assam, Khasia, Sikkim, Nepal, Kumaon, along the Himalayas.

P. (Niph.) subfurfuraceum, Hook. Hab.: Mishmi, Bhotan. \$.

P. (Niph.) nummulariæfolium, Mett. Hab. : Mishmi, Assam;

Khasia, alt. 2,000 feet.

P. (Niph.) floccigerum, Mett. Hab. . Northern India.

P. (Niph.) tissum, Baker (P. porosum, Wall.). Hab.: Assam; Khasia, 5-6,000 feet. Bhotan, Nepal, Kumaon, and North-west Himalaya.

P. (Niph.) flocculosum, Don. (P. detergibile, Hook.). Hab.: chiefly North Bengal, and along the lower range of the Himalaya, from very low altitudes of Silhet and Assam, to 3,500 feet in Kumaon; from Simla, Nepal, Bhotan. \$.

P. (Niph.) Boothii, Hook. Hab. : Eastern Himalaya, Bhotan. \$\$.

P. (Phymatodes) rostratum, Hook. Hab.: Mishmi, Khasia, Bhotan. Hook. Cent. Ferns, t. $53 . \$$.

P. (Phym.) lineare, Thunb. (P. loriforme, Wall.). Hab. : North India; common in all the mountain regions, ascending in the Himalaya from 5-10,000 feet. Hook. Gard. Ferns, t. 14.

P. (Phym.) superficiale, Blume. Hab. : Khasia ; Bhotan, 3-6,000 feet alt.

P. (Phym.) angustatum, Sivz. Hab.: Assam. Hook. Gard. Ferns, t. 20.

P. (Phym.) normale, Don. Hab.: Assam, Khasia; Nepal, 4-6,000 feet. Throughout Northern Bengal.

P. (Phym.) rhyncophyllum, Hook. Hab. : Assam; Khasia, alt. 4,000 feet. Hook. Cent. Ferns, t. 54.

P. (Phym.) Griffithiauum, Hook. Hab.: on trees; Mishmi, Khasia, Bhotan. Hook. Cent. Ferns, t. 51. \$.

P. (Phym.) ovatum, Wall. Hab.: Khasia, Bhotan, Sikkim; Nepal, up to 7,000 feet. Hook. \& Gr. Ic. Fil. t. 41. \$.

P. (Phym.) membranaceun, Don. Hab.: apparently common all over India Proper. Assam, Khasia, Bhotan, Bengal ; Kumaon, alt. 6,500 . India.

P. (Phym.) heterocarpum, Blume. (non Mett.). Hab. : Northern

P. (Phym.) irioides, Lam. Hab.: Assam, Silhet, Bhotan; about Calcutta, and Bengal generally. Hook. Fil. Exot. t. 4.

P. (Phym.) hemionitideum, Wall. Hab.: Nepal, Assam, and Khasia; alt. 4-6,000 feet.

P. (Phym.) pteropus, Blume. (P. tridactylon, Wall.). Hab. : Assam, Khasia, Chittagong. 
Polypodium-continued.

P. (Dipteris) Wallichii, $\boldsymbol{R}$. Br. Hab.: Assam, Khasia, Bhotan, Pundoa mountains. \$.

P. (Phym.) trifidum, Don. (P. oxylobum, Wall.). Hab.: Kumaon, alt. 7,000 feet; thence east to Khasia, alt. 4-5,000 feet.

P. (Phym.) malacodon, Hook. Hab.: North-west Himalaya, alt. 10,500 feet. Kumaon, Simla, Nepal; Silkim, alt. 12-13,000 feet. $\$$.

P. (Phym.) nigrescens, Blume. Hab.: Assam, Silhet. Hook. Fil. Exot. t. 22.

P. (Phym.) dilatatum, Wall. Hab.: Nepal; Khasia, alt. 4-6,000 feet.

P. (Phym.) ebenipes, Hook. Hab. . Nepal, alt. 10,000 feet; North-west Himalaya, alt. 8-9,000 feet; Siklrim, 8,000 feet.

P. (Phym.) longissimum, Blume. Hab.: Assam, Silhet.

P. (Drynaria) conjugatum, Lam. (P. coronans, Wall.). Hab. : Mishmi, Assam; lower hills of TKhasia and Sikkim, 1,500 feet. Nepal, Kumaon (Polypodium contiguum). Hook. Fil. Exot. t. 20.

P. (Dry.) propinquum, Wall. Hab.: Assam; Khasia, alt. 5-7,000 feet. Sikkim, Nepal, Simla; common in the whole Himalayan range (P. Wildenovii). Hook. Gard. Ferns, t. 35.

P. (Dry.) rivale, Mett. MSS. Hab. : NE. Himalayas, \$.

P. (Dry.) quercifolium, Limn. Hab. Himalayas, alt. 1,000 feet.

P. (Phym.) juglandifolium, Don. (P. capitellatum, Wall.). Hab. : Himalaya, Srinagar, Simla, Naini Tal, Kumaon, Nepal, Bhotan; Khasia, 4,000 feet. Sikkim, 7-10,000 feet. \$.

P. (Phym.) Lehmanni, Mett. Hab. : Dorjiling.

P. (Phym.) Himalayense, Hoo\%. Hab.: Nepal, Bhotan, 7,500 feet alt. Sikkim, Khasia.

P. (Phym.) leiorhizon, Wall. Hab.: mountain districts of Kumaon, Nepal, Sikkim, Bhotan; Khasia, up to 5-7,000 feet. Hook, Fil. Exot. t. 25.

\section{No tho chirena.}

‥ Marantæ, R. Br. Hab. . Himalayas, Silkim, and Bhotan, alt. 14-15,000 feet. Kumaon, above Simla.

\section{cymnogramme.}

G. (Leptogramme) Totta, Schlecht. (G. Lowei, Hook. \& Gr. Ic. Fil. t. 89.). Hab.: Kumaon, 4-8,000 feet. Simla, Mahasoo, Assam.

G. (Lept.) aurita, Hook. Hab.: Khasia, Mumbree and Nuncklow; Lachen in Silzlim, alt. 6,000 feet. Hook. Cent. Ferns, t. 74 and $89 . \$ 8$.

G. (Lept.) opaca, Spreng. (G. obtusata, Bl.). Hab.: Nepal, Khasia.

G. (Stegnogramme) aspidioides, Hook. (now Kaulf.). Hab.: Khasia, alt. 6,000 feet. Hook. Cent. Ferns, t. 50.

G. vestita, Hook. Hab.: NW. Himalayas, Simla; Kumaon, alt. $7-8,000$ feet.

G. Andersoni, Beddome. Hab.: Kumaon, alt. 13,000 feet. $\$$.

G. Javanica, Blume. Hab. : all over Ifimalayan India, at an elevation of 7-8,000 feet. Dalhousie, Assam, Khasia.

G. microphylla, Hook. Hab. : on trees at Surureen and Sanatola, Klnasia Hills. Hook. Cent. Ferns, t. 16. \$. 
Gymnogramme-continued. feet.

G. (Selliguea) lanceolata, Hook. Hab. : Himalayas, alt. 3-4,000

G. (Sell.) involuta, Don. Hab. : mountain districts at an elevation of 4-6,000 feet from Kumaon in the west, to Sikkim, Bhotan, and Khasia in the east.

G. (Sell.) Hamiltoniana, Hook. Hab. : East Himalayas, Mishmi, Khasia, Silhet. $\$$.

G. (Sell.) elliptica, Baker (G. decurrens, Wall.). Hab. : Nepal, along the Himalayan range, to Sikkim, Bhotan; Khasia, alt. 6,000 feet.

\section{Brainea.}

B. insignis, Hook. Hab. : Khasia, alt. 4,000 feet.

\section{ris enisclum.}

M. triphyllum, Swartz. Hab.: Himalayas, Silhet, Cachar, Chittagong.

M. cuspidatum, Blume. Hab. : Himalayas.

\section{Antrophyum.}

A. coriaceum, Wall. Hab.: Nepal, on the trunks of trees. Khasia.

A. plantagineum, Kaulf. (A. parvulum, Bl.). Hab.: Khasia, 4,000 feet. Beddome.

A. reticulatum, Kaulf. Hab.: Himalayas.

A. latifolium, Blume. Hab. Bhotan.

\section{Vittaria.}

V. elongata, Swartz. Hab. : Assam, Khasia, Bhotan, Kumaon.

V. (Tæniopsis) lineata, Swartz. Hab. : Himalayas, 3-8,000 feet.

\section{Drymoglossum.}

D. carnosum, Hook. Hab. : Nepal and Sikkim.

D. piloselloides, Presl. Hab.: Himalayas, Silhet; Chittagong, abundant on the mossy trunks of trees.

\section{Hemionitis.}

H. cordata, Roxb. Hab. : rich wet soil about Calcutta (H. cordifolia), Hook. Fil. Exxot. t. 35.

H. Griffithii, Hook. ( fil.) \& Thoms. Hab. Khasia.

\section{Acrostichum.}

A. conforme, Swartz. Hab. : Nepal; Khasia, up to 6,000 feet.

A. viscosum, Swartz. Hab.: Khasia, Nepal.

A. (Stenochlæna) scandens, J. Sin. Hab. . Himalayas, Assam, Silhet, Siklkim, Chittagong.

A. (Egenolfia) appendiculatum, Willd. Hab.: Khasia; Bengal, almost universal in the hilly countries.

A. (Gymnopteris) variabile, Hook. Hab. : Assam; Khasia, alt. 2-3,000 feet.

A. (Gym.) Hagelliferum, Wall. Hab.: Nepal, Assam, Chittagong.

A. (Gym.) virens, Wall. Hab.: Himalayas, Assam, Khasia, Bhotan, Siklim, Nepal, Silhet, Chittagong, Kumaon.

A. (Chrysodium) minus, Mett. Hab. : Khasia, near Churra, alt. $2-3,000$ feet.

A. (Chrys.) axillare, Cav. Hab.: Assam, Silhet, Bhotan. 
Acrostichum-continued.

A. (Chrys.) tricuspe, Hook. Hab. : hot valleys of SikkimHimalaya. $\$$.

A. (Chrys.) Blumeanum, Hook. Hab.: Assam.

A. (Hymenolepis) spicatum, Linn. Hab.: Himalayas, to 6,000 feet. Assam, Ihhasia, Churra, and Sikkim. Hook. Fil. Exot. t. 78; Hook. Gard. Ferns, t. 3.

\section{osmunda.}

O. Claytoniana, Linn. (O. interrupta, Michx.; O. pilosa and monticola, $H^{2}(l l l$.). Hab. : Himalayas, up to 10,000 feet. Khasia hills. O. regalis, Linn. (O. speciosa, Wall.). Hab. : Himalayas.

\section{Schizza.}

S. (Actinostachrs) digitata, Sw. Hab. . Himalayas, Khasia. Hook. Gard. Ferns, t. 49.

\section{Iygodium.}

L. scandens, Swz. Hab.: Himalayas.

L. pinnatifidum, Swz. Hab.: Himalayas.

L. japonicum, Swz. Hab.: Himalayas.

\section{Anglopteris.}

A. evecta, Hoffm. Hab. : Sikkim, elevation, 1,000 feet. Assam and Khasia. Hook. Fil. Exot. t. 75.

\section{Kaulfussia.}

\section{Assam.}

K. æsculifolia, Blume. (K. Assamica, Griff.). Hab. : Upper

\section{Ophioglossum.}

O. vulgatum, Linn. Hab.: Himalayas.

O. reticulatum, Lim. Hab. : Himalayas.

O. (Ophioderma) pendulum, Lim. Hab. : Assam.

\section{Helminthostachys.}

H. zeylanica, Hook. Hab.: Himalayas.

\section{Botrychium.}

B. Lunaria, Su: Hab.: Himalayas.

B. daucifolium, Wrll. Hab.: Himalayas.

B. virginianum, Sic. Hab.: Himalayas, up to 6,000 feet. Khasia. Hook. Gand. Ferns, t. 29. 


\section{SOUTHERN INDLA (INCLUDING CONCAN, DEC- CAN, ORISSA, UP TO THE $20^{\circ}$ OF $\mathrm{N}$. LAT. CEYLON).}

\section{Gleichenta.}

G. dichotoma, Trilld. Hab.: Malabar coast, with rigid fronds. Nilghiris, Concan, Canara; very common from the sea-level on the western coast to the tops of the highest mountains; ravines on the North Arcot hills. Beddome.

\section{Cyathea.}

C. sinuata, Hook. \& Gr. Hab.: wooded mountains, Ceylon; forests in the Kokooles and Hinidoon Corles, at no great elevation; most abundant in the Singbe-rajah forest; a native only of Ceylon. Hook. \& Gr. Ic. Fil. t. 106; Hook. Gard. Ferns, t. 21. \$.

C. Hookeri, Thw. Hab. : forest of Singhe-rajah, Ceylon. \$.

C. spinulosa, Wall. Hab. : Madras, Shovaghiri bills.

\section{Eemitelia.}

H. (Amplicosmia) Walkeræ, Hook. Hab. : Ceylon; common in the Central Province, Adam's Peak, to an alt. of 6,000 feet. \$.

\section{Alsophila.}

A. crinita, Hook. Hab.: Nilghiris, a ravine on the road from Ootacamund to the Davie Shola; Sisparah ghât, common; higher ranges of Animalayas (Malabar); Ceylon, Newera Ellia, and other of the more elevated parts of the Central Province.

A. glabra, Honk. (A gigantea, Wall.). Hab.: Ceylon; not uncommon in the Central and Southern Provinces, up to an elevation of 4,000 feet.-Thwaites. Malabar, Wynad, Animalayas; Kurg, up to 4,000 feet elevation. Beddome.

A. latebrosa, Hook. Hab.: Nilghiris, Pulneys, Animalayas, Kurg, Shiwaris. The common Tree-Fern. Beddome.

\section{Blacalpe.}

D. aspidioides, Bl. Hab. : Ceylon. Black Pool in Newera Ellia. Thwoaites.

\section{Spheeropteris.}

S. barbata, Wall. Hab. : western ghâts of the Nilghiris; rare.

\section{Dicksonia.}

D. scabra, Wall. (D. deltoidea, Hook.). Hsb.: Ceylon, most elevated parts of the Central Province.

\section{Eymenophyllum.} drict. $\$$.

H. corticola, Hook. Hab. : Ceylon, on trees; Ambagamowa dis-

H. exsertum, $\Pi^{2}$ all. Hab.: Ceylon; very common in the upper forests of the Central Province.-Thwaites. In Malabar, common on trees and moist rocks on the higher ranges of the Nilghiris, Animalayas and Pulneys. Beddome.

H. polyanthos, Swartz. Hab.: Ceylon; upper forests of the Central Province. Thwaites.

H. Javanicum, Spreng. (H. crispatum, Wall.). Hab.: Ceylon : very abundant in the forests of the Central and Southern Provinces.- 
Hymenophyllum-continued.

Thwaites. Common in moist forests on the Nilghiris, Animalayas ; Wynad, up to 4,000 feet elevation. Beddome.

H. Tunbridgense, Sm. Hab. : Ceylon, common in the more elevated parts of the Central Province. Thwaites.

\section{Trichomanes.}

T. Nilghirense, Beddome. Hab. : abundant about Walaghat, on the western slope of the Nilghiris. $\$$.

T. muscoides, Swz. Hab.: Ceylon; Raxawa, in the Central Province; Palagalla. Thwaites.

T. proliferum, $B l$. Hab.: Ceylon, Ambagamowa district, westem slope of the Nilghiris.

T. intramarginale, Ilook. \& Gr. Hab. . Ceylon, Ambagamowa district, Malabar, Shevaghiris. $\$ 8$.

T. pallidum, Bl. (T. glauco-fuscum, Hook.; T. album, Bl.). Hab.: Ceylon, Ambagamowa district.-Theraites. Common in the forests of the Central and Southern Provinces.

T. rigidum, Sirz. (T. strictum, Hook. \& Gr. Ic. Fil. t.122.). Hab.: Ceylon, upper forests of the Central Province; in very moist dark localities on the Nilgbiris and Animalayas, 3-4,000 feet elevation; abundant about Walaghat, down the Sisparah ghât. Beddome.

\section{Davallia.}

D. (Humata) pedata, Sinith. Hab.: Ceylon, common in the Central Province; on the western slopes of the Nilghiris, rare. Beddume; Hook. Gard. Ferres, t. 7.

D. (Hum.) vestita, Bl. Hab.: Ceylon, Bopatalawa; plains in the Central Prorince, at an elevation of 5-6,000 feet. Thwaites.

D. (Lencostegia) chrernphylla, Wall. Hab.: Neddiwattam, on the Nilghiris; common in the teak forests of the Animalayas, growing on trees; Wynad, Coimbatore hills.-Beddome. Ceylon; forests above Galagama, upon rocks in deep gullies. Thwaiters.

D. (Leucos.) affinis. Hook. Hab. . Ceylon, very common in damp forests of the Central l'rovince.-Thicates. Nilghinis.

D. (Odontoloma) repens, Desv. (D. Boryana, Presl.). Hab. : Nilghiris. Ceylon; Singbe-rajah forest.

D. (P'rosaptia) Emersoni, Hook. \& Gr. Hab.: Madras, Animalaya hills; Palghat hills, a rare fern.-Beddome. Ceylon, not uncommon in forests of the Central Province. Hook. \& Gir. Ic. Fil. t. 105.

D. (Prosap.) contigua, Sw: Hab. Animalayan hills, on trees and rocks, on the banks of the Toracadno river, at an elevation of 4,500 feet, a rare fern. Beddume; Mook. \& Gr. Ic. Fil. t. 141.

D. solida, Sicz. Hab.: Maldive Islands.

D. elegans, Sir IIab. : Madras, Tranquebar, Mysore; Malabar, not common.-Beddome. Ceylon; not very uncommon in the Central and Soutbern Provinces of the island, at no great eleration. Thwaites.

D. bullata, $\Pi^{*}$ all. Hab.: Animalayas, on rocks and trees, 34,000 feet elevation. Wywad, a rare fern. Beddome.

D. (Nicrolepia) pinnata, Ćav. Hab.: Animalaya hills, rare. Beddome.

D. (Alicro.) villosa, Wall. Hab.: Ceylon. 
Darallia-continued.

D.(Micro.) strigosa, Swz. (D. Khasyana, Hook.). Hab. : Ceylon, common in the forests of the Central Province.-Thwaites. Chokampatty hills (Tinnavelly district), at an elevation of 5,000 feet. Beddome.

D. (Micro.) platyphylla, Don. (D. Ionchitidea, Wall.). Hab. : Madras, Ceylon; Rambodde, in the Central Province. Thwaites; Hook. Fil. Exot. t. 19.

D. (Micro.) Thwaitesii, Baker. Hab. : Ceylon. \$\$.

D. (Micro.) inæqualis, Kunze. Hab. : Ceylon. Thwaites.

D. (Micro.) hirta, Kaulf. Hab.: Ceylon ; forests of the Central Province, common.

D. (Micro.) Speluncæ, Baker (D. polypodioides, Hook.). Hab.: Ceylon; very abundant in the Southern and Central Provinces. - Thwaites. Madras; Nilghiris, common; Davie Shola, and in ravines on Dodubett, Coonoor, ravines on the Gbât, Animalayas, Pulneys, Travancore hills. Beddome.

D. (Stenoloma) tenuifolia, Swz. Hab. : Ceylon; very abundant. Madras; Coonuor, very common; Coimbatore hills, at an elevation of 3,000 feet only. Animalayas, Kurg. Beddome.

\section{Iindsaya.}

L. cultrata, Swz. Hab.: Nilghiris, very common on the banks of streams. Ceylon, abundant in the more elevated parts of the Central Province. Hook. \& Gr. Ic. Fil. t. 144; Fil. Exot. 67.

I. flabellulata, Dry. Hab. : Ceylon, forests of the Central Province.-Thwaites. (L. tenera, Dry.) Travancore hills, a rare fern. Beddome.

L. trapeziformis, Diy. (L. caudata, Hook.). Hab.: Ceylon; Adam's Peak, forests of the Central Province, not uncommon. Thwoaites; Hook. Cent. Ferns, t. 58.

L. Walkeræ, Hook. Hab.: Ceylon; swampy grassy places in the Saffragam district. Thwaites.

L. nitens, $B$. (L. recurvata, $\Pi^{r} a h$.). Hab. : Nilghiris. Ceylon, up to 5,000 feet; very common in the forests of the Central Provinces. Bolampatty valley, Coimbatore hills. Bedrlome.

L. ensifolia, Swz. Hab.: Ceylon, common in the warmer parts of the island.-Thwaites. Foot of the Sisparah and Carcoor ghâts in Malabar. Beddome; Hook. Gard. Ferns, t. 62.

L. heterophylla, Dry. Hab.: Ceylon. Nilghiris, Bolampatty ralley; Coimbatore hills, rare. Shiwari hills; ravines on the green hills. Beddone.

\section{Adiantum.}

A. lunulatum, Burm. Hab.: very common throughout the Madras Presidency; low mountainous tracts on the eastern side, and from the sea-level up to about 4,000 feet on the western side. -Beddome. Ceylon, hotter parts of the island. Thwaites.

A. caudatum, Linn. Hab.: Malabar, Nilghiris, Ceylon; very abundant in the warmer parts of the island. Oova and Doombera districts. Thwaites.

A. Capillus-veneris, Limn. Hab.: Ceylon. Oova and Doombera districts.-Thwaites. Nilghiris, rare. Coimbatore plains, banks of a river. Beddome. 
Adiantum-continued.

A. Ethiopicum, Linn. Hab. : Nilghiris; Ootacamund, Dodabetta, Neddiwattam. Pulneys, by no means a common fern. Beddome.

A. hispidulum, Swuz. Hab. : Nilghiris, Pulney hill ; Animalayas, rather rare. Ceylon, not uncommon.

A. Habellulatum, Linn. Hab.: Ceylon, Oova district. Thwaites.

\section{Cheilanthes.}

Ch. Mysorensis, Wall. Hab. : one of the most common ferns in the Madras Peninsula; found among rocks and hills, at all heights, from 50-3,000 feet above the level of the sea.-Dr. Wight. Ceylon; Newera Ellia, 6,000 feet. Badulla and Doombera districts. Thwaites.

Ch. varians, Hook. Hab.: Ceylon, Kornegalle district, and Caloopahane.-Thwaites. Malabar; Animalaya mountains, rare. Beddrme.

Ch. Dalhousiæ, Hook. Hab. : Nilghiris, in woods near Makoorty.

Ch. bullosa, Kunze. Hab. : Nilghiris, very common. \$.

Ch. tenuifolia, Swartz. Hab.: Ceylon, common in the warmer parts of the island.- Thwaites. Found in dry localities all over the Madras Presidency : it is not found at any great elevation.

Ch. myriophylla, Desv. Hab. : Nilghiris.

Ch. farinosa, Kaulf. Hab. : Bombay, Nilghiris; Ceylon, more elevated parts of the Central Province.

\section{onychium.}

O. auratum, Kaulf. Hab.: Palghat mountains; Madras Presidency, rare. Beddome.

\section{Pellzea.}

P. geraniæfolia, Fée. Hab. . Nilghiris; very common on the western side, up to an elevation of 5,000 feet. Hook. Cent. Ferns, t. 15 .

P. Boivini, Hook. Hab.: Nilghiris; abundant on the Sisparah ghât, dry places, 3-5,000 feet elevation. Animalaya mountains, on dry rocky places, 4,000 feet elevation.

P. falcata, Fée. Hab.: Animalayas, in moist woods, elevation, 3-4,000 feet; Pulney hills, Nilghiris, Droog hill, and rarines on the Coonoor ghât; Lampen's Peals, near Coimbatore. Beddome.

\section{Pteris.}

Pt. longifolia, Linn. Hab. : Ceylon, Oova and Doombera districts.-Thwaites. Banks of a river near Coimbatore. Beddome.

Pt. cretica, Limn. Hab.. Ceylon, Oova district; very common on the higher ranges of the Animalayas, Pulneys and Nilghiris.

Pt. pellucida, Presl. Hab. : Ceylon, Nilghiris, Animalayas; forests, $2-4,000$ feet elevation.

Pt. Hookeriana, Agardh. Hab.: Adam's Peak; Ceylon, at about 2,000 feet; Saffragan district, Nalabar coast ; Cochin, rare. $\$$.

Pt. crenata, Swartz. Hab. : Cochin, Nalabar coast, Tranquebar ; Ceylon, abundant in the warmer parts of the island. Thwaites.

Pt. semipinnata, Liun. HIab.: Travancore hills, Ceylon, Tranquebar. Hook. Gard. Ferns, t. 5 9.

Pt. quadriaurita, Retz. Hah. : Nilghiris, very abundant everywhere in the western side of the Madras Presidency; Ceylon. 
Pteris-continued. hills.

Pt. longipinnula, Wall. Hab. : Madras, foreats on the Travancore

Pt. pellucens, Agardh. Hab.: Animalayas, 2,500 to 4,000 feet; Coimbatore hills; Carcoor ghât, by no means common. Beddome.

Pt. aquilina, Linn. Hab.: Mahabaleshwar, Concan, Nilghinis; all over the hilly portions of the west.

Pt. biaurita, Linn. Hab.: Concan, Nilghiris; Ceylon, very common in the Central Province.

Pt. patens, Hook. Hab.: Ceylon; forests of the Central Province, not very uncommon.-Thwaites. New Carcoor ghât (Wynad), abundant. Beddome.

Pt. palmata, Willd. (Pt. pedata, Linn.). Hab.: Dindigal mountains, Nilghiris. Hook. Fil. Exot. t. 34 .

Pt. incisa, Thunb. (Pt. aurita, Bl.). Hab.: Ceylon, not uncommon in the Central Province.

Pt. tripartita, Swartz. Hab. . Ceylon, forests of the Central Province; not very common. Thwaites.

\section{Ceratopteris.}

C. thalictroides, Brogn. Hab. . Ceylon, not uncommon on the borders of streams and of paddy fields. - Thwaites. Common in swampy places in the plains and subalpine jungles.

\section{romaria.}

L. Patersoni, Spreng. (L. elongata, Blume.). Hab.: Ceylon and the Nilghiris ; very abundant on the Koondah mountains. Sisparah ghât, all the ravines, above 4,000 feet elevation; Animalayas. Beddome.

\section{Blechnum.}

B. orientale, Linn. Hab.: Ceylon, Madras; common on the Nilghiris, Animalayas, Pulneys, and Coimbatore hills; Beypore and Calicut (Malabar plains). Beddome.

\section{Doodia.} $\$$ \$.

D. dives, Kunze. Hab. : Ceylon, wood of the Central Province.

\section{Asplenium.}

A. Nidus, Linn. Hab. : Ceylon, very common.-Thwaites. IEoist woods on the Animalayas, 3-4,000 feet elevation, very abundant. Beddone.

A. ensiforme, Wall. Hab.: Ceylon; Rambodda, in the Central Province.-Thwaites. Animalayas; banks of the Toracadu river, 4,500 feet elevation, rave. Hooǩk. \& Gr. Ic. Fil. t. 71 .

A. Trichomanes, Linn. Hab.: Kalhutty, on the Nilghiris, between 5-6,000 feet elevation. Beddome.

A. normale, Don. (A. multijugum, Wall.). Hab. Ceylon, heights of Peradenia; Nilghiris, and Animalayas, common on the higher elevations.

A. Wightianum, Wall. Hab. : Ceylon, common in the forests of the Central Province ; Animalayas; Bolamputty valley, in the Coimbatore hills; Pulney mountains, on rocks and trees in moist forests; on the banks of rivers, $2-4000$ feet elevation. Beddome. 
Asplenizm-continued.

A. tenerum, Forst. Hab. . Ceylon; Hantani, in the Central Province.

A. erectum, Bory. Hab.: Ceylon; Central Province, not uncommon.-Thwaites. Nilghiris, about Utacamund.

A. persicifolium, J. Sm. Hab. : Ceylon, Newera Ellia, and other more elevated parts of the Central Province.-Thavaites. Nilghiris and Animalayas.

A. contiguum, Kaulf. Hab. : Nilghiris, 7,000 feet; Wynad. Animalaya hills, 3,000 feet elevation.

A. auritum, Show. Hab.: Nilghiris; Coonor, on rocks, rare. Animalaya rocks in the bed of the Toracadu river, 4,500 feet elevation. Beddome.

A. falcatum, Lam. Hab.: Ceylon, Courtallam and Travancore; Animalaya forests, on rocks 3,500 feet.

A. caudatum, Forst. Hab.: Ceylon, Newera Ellia.

A. macrophyllum, Swz. Hab.: Ceylon, Newera Ellia, and other more elevated parts of the Central Province-Thwaites. Animalaya forests, on rocks and trees, 2-4,000 feet elevation. Nilghiris. Beddome.

A. formosum, IFilld. Hab.: Ceylon, on mountains, elevation 3,000 feet; forests abore Galagana, towards Horton Plains.Thwites. Nilghiris, very abundant in moist woods at the higher elevations. Beddome.

A. resectum, Smith. Hab.: Ceylon, very abnndant in the forests of the Central Province. Nilghiris, Animalayas; Pulneys, very common at an elevation of 3-5,000 feet. Hook. \& Gr. Ic. Fil. t. 114 .

A. heterocarpum, $T^{\top} a l l$. Hab. : Ceylon, not uncommon in mist woods.-Thwaites. Travancore hills, Hereara, Nilghiris (Avalanche and Neddiwattan ). Beddome.

A. planicaule, Wall. Hab.: Ceylon, not uneommon in forests of the Central Province.-Thuaites. Very common in mountainous tracts on the western side of the Madras Presidency. Bumbay, Mahableshwar.

A. furcatum, Thunb. Ilab.: Ceylon, on mountains 7-8,000 feet eleration, in the Central Province. Abundant about Utacamund in the Nilghiris, and on the higher ranges of other lofty mountains on the western side of the Madras Presidency.

A. affine, Swa. (A. spathulinum, J. Sm.). Hab.: ('eylon, on Newera Ellia. Central Province, in the higher forests. Thwaites.

A. nitidum, Sur. Hab.: Ceylon, common in the forests of the Central Province.-Thwaits. Tranquebar. Sisparah ghât, on the Nilghiris ; 4,000 feet elevation. Beddome.

A. fontanum, Bernh. Hab. . Nilghiris, rare; on the banks of the river above the Kalhatty waterfall; growing on rocks. Beddome.

A. varians, Hook. \& Gr. Hab. : Ceylon; Oova district, not common.-Thwaites. Nilghiris, about Utacamund. Beddome; Hook. \& Gr. Ic. Fil. t. 172.

A. tenuifolium, Dan. (A. concinnum, Wall.). Hab.: Ceylon; Newera Ellia, by the sides of the streams. - Thurites. Nilghiris, 
Asplenium-continued.

common on the banks of streams at Utacamund, Animalayas. Beddome; Hook. Second Cent. Ferns, t. 29.

A. (Darea.) rutæfolium, Kunze. (A. prolongatum, Hook.). Hab.: Ceylon, Pas-doon Corle; Singhe-rajah forest.-Thwaites. Shevaghiri mountains (Malabar), rare. Hook. Second Cent. Ferns, t. 42.

A. (Athyrium) Hohenackerianum, Kunze. Hab.: Ceylon, Nilghiris, Animalayas, Wynad; not uncommon. Jungles about Palghat. Concan, Kurg, and South Canara. Very abundant from the plains up to 4,000 feet. Beddome.

A. (Athyr.) macrocarpum, Blume. Hab. : Ceylon, more elevated parts of the Central Province--Thwaites. Nilghiris, Animalayas.

A. (Athyr.) nigripes, Blame. Hab. : Ceylon; forests of the Central Province, at an elevation of 4-6,000 feet.-Thwaites. Nilghiris.

A. (Athyr.) Filix-foemina, Bernh. Hab. : forests on the north bank of the Godavery. (Var. A pectinatum, Wall.) Nilghiris; very abundant about Utacamund, banks of streams by roadsides. Beddome.

A. (Athyr.) aspidioides, Schlecht. Hab.: Nilghiris, Ceylon, Newera Elia, Maturatte, etc. Thwaites.

A. (Athyr.) umbrosum, f. Sm. (A. australe, Bracl.). Hab.. Nilghiris, about Utacamund and Neddiwattan; Mysore. Ceylon, damp shady forests in the Central Prorince.

A. (Diplazium) lanceum, Thunb. Hab.: Ceylon, forests of the Central Province. Thwaites.

A. (Dipl.) Zeylanicum, Hook. Hab. : Ceylon, forests of the Ambagamowa and Kotmalie districts. Thwaites; Hook. Second Cent. Ferns, t. $16 . \$$

A. (Dipl.) sylvaticum, Presl. Hab.: Ceylon, common in the forests of the Central and Southern Provinces.-Thwaites, Nilghiris, abundant down the Sisparah ghât.

A. (Dipl.) japonicum, Thunb. (A. Schkuhrii, Hook.). Hab.: Ceylon, Ambagamowa and Saffragam districts. Thwaites.

A. (Dipl.) Thwaitesii, A. Br. Hab. : Ceylon, forests of the Central Province. Hook. Second Cent. Ferns, t. 45 . $\$$.

A. (Dipl.) lasiopteris, Mett. Hab. : Nilghiris, near Utacamund, rare; Pulney Hills, abundant about Poombarry. Beddome.

A. (Dipl.) polypodioides, Mett. Hab.: Ceylon, very abundant in damp forests. - Thwaites. Nilghiris, very abundant. In some parts of the Animalayas it is quite a tree-fern. Beddome.

A. (Dipl.) maximum, Don. (A. dilatatum, Hook.). Hab. : Ceylon, very common in damp forests. Nilghiris.

A. (Aniso.) esculentum, Presl. Hab.: Ceylon, common on the shady banks of rivers and streams. - Thwaites. Very common in the Nilghiris and Animalayas, on banks of streams, at no great elevation. Beddome.

A. (Aniso.) Smithianum, Baker. Hab.: Ceylon. \$.

\section{Allantodia.}

A. Brunoniana, Wall. Hab. : Ceylon; damp forests of the Central Province, at an eleration of 3-6,000 feet.-Thwaites. Ghâts, in Canara, rare. Beddome.

\section{Actinopteris.}

A. radiata, Link. Hab. . Bombay, old walls; Madras, common in 
Actinopteris-continued.

diy rocky places, from the sea-level up to $3-4000$ feet.-Becdome. Ceylon, Trincomalie; Gonagama, on the lower Badulla road from Kandy. Thwaites; Hools. Cent. Ferns, t. 75.

\section{Aspidium.}

A. (Polyst.) auriculatum, Swz. Hab.. Ceylon, common in the forests of the Central Province. Very common on the higher ranges of the Nilghiris.

A. (Polyst.) aculeatum, Swz. Hab.: Ceylon, more elerated parts of the Central Province.-- Thwaites. About Utacamund, and the higher elevations of the Pulneys and Animalayas. Beddone.

A. (Polyst.) anomalum, Hook. \& Arn. Hab.: Ceylon, Horton Plains, and Happootelle, at an elevation of 5-6,000 feet. Thoaites. \$.

A. (Polyst.) amabile, Blume. Hub.: Ceylon, more elevated parts of the Central Province; Nilghiris, Pulney hills; Animalays, not common.

A. (Polyst.) aristatum, Sws. Hab.: Ceylon, very common in the Central Province. Nilghiris.

A. (Cyrt.) falcatum, Swz. Hab. : Nilghiris, about Utacamund.

A. membranaceum, Hook. Hab.: Ceylon.

\section{Nephrodium.}

N. (Lastrea) cuspidatum, Baker (Polyp-elongatum, Wall.). Hab.: Ceylon, Carawitte, Kanda, and Ambagamowa. Thwitites.

N. (Last.) hirtipes, Hook. Hab.: Ceylon, common in the upper forests of the Central Province. Nilghiris, vely abundant, 4,000 feet and upwards. Animalayas, Pulneys. Beddome.

N. (Last.) gracilescens, Hook. Hab.: Ceylon, more elevated parts of the Central Province. Travancore hills, rather rare.

N. (Last.) calcaratum, Hook. (N. falcilobum, Hook.). Hab. : Ceylon, on the sides of streams and in damp forests; common. Nilghiris, in ravines about half-way down the Sisparah ghât. Animalayas, beds of rivers, 2-4,000 feet elevation.

N. (Last.) Beddomei, Baker. Hab. : Nilghiris, Ceylon.

N. (Last.) prolixum, Baker (N. octhodes, Hook.; A. tylodes, Kze.; N. appendiculatum, Hook.). Hab.: Ceylon, Newern Ellia, and other more elevated parts of the Central Province.-Thwaites. Niluhiris, rather rare. Beddome.

N. (Last.) griseum, Baker: Hab.: S. Hindostan.

N. (Last.) syrmaticum, Baker (N. spectabile, Hook.). Hab.: Ceylon, higher forests of the Central Province.-Thwaites. Nilghiris, Carcoor ghât (Wruad). Animalayas, rare; ravines in the north Arcut hills, near Kirkumbaddy.

N. (Last.) Filix-mas, Rich. Hab.: Ceylon, Newera Ellia, and other of the most elerated parts of the Central Province.-Thuvites. Animalayas; Nilghiris, rocky hills, 5,000 feet and upwards. Beddome.

N. (Last.) flaccidum, Hook. Hab.: Ceylon, more elevated parts of the Central Province. Nilghiris, abundint on the Carcoor ghât. Animalayas, 3,000 feet elevation. Beddome.

N. (Last.) sparsum, Don. (N. purpurascens, Hook.). IIab.: Nilghiris, Ceplon; very abundant in the forests of the Central Province. Thwaites. 
Nephrodium-continued.

N. (Last.) undulatum, Baker. Hab. : Ceylon. \$.

N. (Last.) deparioides, Hook. Hab. . Ceylon; forests of the Central and Southern Provinces, up to an elevation of 3,000 feet.Thwaites. Animalaya hills, rare. Hook. Fil. Exot. t. 3.

N. (Last.) Thwaitesii, Baker. Hab.: Ceylon, Singhe-rajah forest. $\$$.

N. (Last.) odoratum, Baker (Neriocarpum, Desv.). Hab.: Animalayas, on the Perigoonda hill, 5,000 feet elevation,-Beddome. Mahableshwar.

N. (Last.) recedens, Hook. Hab.: Ceylon, forests of the Central Province ; Nilghiris, Pulney mountains, 4,000 feet elevation ; a rare fern.

N. (Last.) membranifolium, Presl. Hab.: Ceylon, common in forests of the Central Province. Nilghiris, Sisparah, and Carcoor ghâts, Wynad, Animalayas.

N.' (Last.) ferrugineum, Baker. Hab. : Nilghiris. \$.

N. (Last.) scabrosum, Baker. Hab. : Nilghiris. \$\$.

N. (Last.) intermedium, Baker. Hab. . Ceylon; Nilghiris, near Malee Mand; Animalayas, 5,000 feet.

N. (Last.) obtusifolium, Baker. Hab.: Ceylon, at Newera Ellia, and Maturatte. \$.

N. (Last.) Boryanum, Baker (non Hook.; N. divisum, Wall.). Hab.: Ceylon, forests at Roxawa and Happootelle, in the Central Province.-Thwaites. Nilghiris, about Utacamund, 7,000 feet elevation. Animalayas, 3,000 feet elevation. Beddome.

N. (Last.) setigerum, Baker (N. tenericaule, Hook.). Hab.: Ceylon, abundant in the Central Province.

N. Otaria, Baker (N. aristatum, Hook.). Hab. . Animalayas; abundant in the teak forests, 2-4,000 feet elevation. Ceylon, Bintenne; Cronagama, north of Kandy. Thwaites.

N. unitum, $R . B r$. (N. propinquum, $B r$.). Hab. : Ceylon, common; Nilghiris and Animalayas, 3,000 feet in the Karambevile (a large swamp).

N. pteroides, J. Sm. (A. terminans, Wall.). Hab.: Ceylon, Central Province, not uncommon.-Thwaites. Nilghiris, Animalayas; in moist forests, $2-4,000$ feet elevation.

N. extensum, Hook. Hab.: Ceylon, Oova district; Nilghiris, Animalayas, and other mountainous tracts.

N. cucullatum, Baker (N. Unitum, Sieb.). Hab.: Ceylon.

N. Haenkeanum, Presl. Hab. : Ceylon.

N. Arbuscula, Deso. Hab.: Ceylon, common in the Central Province.-Thwaites. Nilghiris, Animalayas, and Pulneys; beds of rivers, at 3-4,000 feet. Beddome; Hook. Second Cent. Ferns, t. 22.

N. abortivum, J. Sm. Hab.: Ceylon.

N. pennigerum, Hook. Hab. : Ceylon.

N. molle, Desv. Hab. : Ceylon, very abundant throughout the island.-Thwaites. And one of the commonest ferns in India, everywhere.

N. truncatum, Presl. (N. eusorum, Thwaites; N. abruptum, Presl.). Hab. : Ceylon, forests of the Central Province, not uncommon; Nilghiris, Animalayas.

N. (Sagenia) polymorphum, Baker. Hab. . Ceylon, common in 
Nephrodium-continued.

the Central Province.-Thwaites. Nilghiris, very common in ravines on the Cnonoor ghât, Pulney hills. Beddome.

N. (Sagen.) subtriphyllum, Baǩes. Hab. . Ceylon; forests of Allagalla and Maturatte, in the Central Province.-Thwaites. Nilghiris.

N. (Sagen.) decurrens, Baker (Aspid. pteropus, Kze.). Hab.: Ceylon, common in damp forests ; Coimbatore hills, Madras Presidency.

N. (Sagen.) cicutarium, Baker. Hab. : Ceylon, Central Province; Nilohiris, very abundant.

N. (Sagen.) giganteum, Baker. Hab.: Ceylon, very abundant in the Central Province-Thwaites. Animalayas, Malabar.

\section{Nephrolepis.}

N. cordifolia, Baker (N. tuberosa, $\boldsymbol{P r}$.). Hab. : Ceylon, common throughout the island, and in subalpine jungles of the Madras Presidency.

N. exaltata, Schott. Hab. : Ceylon, very abundant on the margins of wuods, sides of streams, \&c.-Thwaites. Nilghiris, Animalayas, Wynad.

N. acuta, Presl. IIab. : Ceylon, equally abundant with N. exaltata.-Thwaites. Ravines near Kirkumbaddy, North Arcot Hills. Beddome.

N. ramosa, Baker (N. obliterata, Hook.). Hab. . Ceylon; a very common plant. Thwaites.

\section{oleandra.}

O. neriiformis, Cav. Hab.: Ceylon, upper forests of the Central Province, western slopes of Nilghiris, Animalayas; rare.

O. musefolia, Tunze. Hab.: Ceylon.

O. Cumingii, J. Sne. Hab.: Nilghiris.

\section{Polypodium.}

P. (Pheg.) Walkeræ, Hook. Hab. : Ceylon; Adam's Peak, alt. 6,000 feet. \$s.

P. (Pheg.) distans, Don. (P. paludosum, Bl.). Hab.: Ceylon, more elerated parts of the Central Prorince.-Thwaites. Very common about Utacamund, on the Nilghiris. Beddome.

P. (Pheg.) rufescens, Blume. Hab. : Ceylon, forests of the Central Province, at an elevation of :j-5,000 feet. Thwaites.

P. (Pheg.) punctatum, Thunb. Hab.: Ceylon.

P. (Goniopteris) urophyllum, Thall. Hab. : Ceylon; forests of the Central Province, not uncommon Thurates.

P. (Goniopt.) proliferum, Presl. Hab. : Nilghiris. Hook. Second C'ent. Ferns, t. 15.

P. (Goniopt.) lineatum, Colcbr. Hab.: Ceylon.

P. (Dictyopteris) difforme, Lime. HIab.: Animalaya furests, Madras. Beddome.

P. parasiticum, Mett. Hab. : Nilghiris; Ceylon, Central Proviuce, and in Pas-doon-Coola. Thurres.

P. hirtellum, Bl. (P. Iasiosorum, IIook.). Hab.: Ceylon, Bngawantalawa; Central Provinces, 5,000 feet elevation, on trees. Beddone. 
Polypodium-continued.

P. Zeylanicum, Mett. Hab.: Ceylon, common in the more elevated parts of the Central Province. Thwaites. $\$$.

P. cucullatum, Nees. Hlab. : Rhamboda, Ceylon; higher forests of the Central Province, not common. Thwaites.

P. parvulum, Bory. Hab.: Ceylon; common on the trunks of trees in the higher parts of the Central Province.-Thurites. Animalayas, on rocks; banks of the Toracadu river, 4,500 feet elevation; Nilghiris, near Coonoor. Beddome.

P. glandulosum, Hook. IIab. : Ceylon; Rambodde, in the Central Province. Thwaites.

P. obliquatum, Blume. Hab.: Ceylon; very abundant on the trunks of trees in the more elevated parts of the Central Province. Shevagiri mountains, Malabar.

P. repandulum, Mett. Hab.: Ceylon, Central Province. \$.

P. minutum, Blume. Hab.: Ceylon.

P. subfalcatum, Blume. Hab.: Ceylon, Animalaya hills. Beddome.

P. fuscatum, Blume. Hab. : Ceylon.

P. decorum, Brack. Hab.: Ceylun, Singhe-rajah forest, and in the Hinidoon Pattoo. Thwaites.

P. (Niphobolus) adnascens, Swz. Hab.: Ceylon, upon the trunks of trees.-Thwaites. Madras, common in all the western coast forests from the plains up to 5,000 feet. Concan. Hook. Gard. Ferns, t. 19.

P. (Niph.) acrostichoides, Forst. Hab.: Ceylon.

P. (Niph.) Lingua, Suz. Hab. : Ceylon; not uncommon in the warmer parts of the island, and up to an elevation of 3,000 feet. Thwaites.

P. (Niph.) nummulariæfolium, Mett. Hab.: Nilghiris; common in the lower forests of the Animalayas, Wynad, Kurg, \&c.; parasitic on trees. Beddome.

P. (Niph.) fissum, Baker (P. porosum, Waill.). Hab. : Ceylon; forests of the Central Province, at no great elevation, not common. -Thwaites. Nilghiris, abundant; on all mountainous tracts on the western side, from $1-8,000$ feet. Beddome.

P. (Niph.) Gardneri, Mett. Hab.: Ceylon, not uncommon. Hook. Fil. Exot. t. 68.

P. (Phymatodes) lineare, Thunb. (P. Wightianum, Wall.; P. loriforme, Wall.). Hab. : Ceylon, common in forests of the Central Province. Common in the hilly regions of the Madras Presidency. Hook. Gard. Ferns, t. 14.

P. (Phym.) lanceolatum, Lim. (P. lepidotum, Tilld.). Hab.: Nilghiris, common in woods about Utacamund. Beddome.

P. (Phym) angustatum, Suz. Hab.: Tranquebar; Nilghiris, very rare. Hook. Gard. Ferns, t. 20.

P. (Phym.) membranaceum, Don. Hab.: Ceylon; common on the trunks of trees, \&c., in damp forests of the Central Province. -Thwaites. Nilghiris; on trees near Neddiwattan, Animalayas, from 2,000 feet upwards. Wynad, Curg, South Canara ghâts. Beddome.

P. (Phym.) heterocarpum, Blume. (non Mstt.). Hab.: Ceylon. 
Pulypodium-continued.

P. (Phym.) irioides, Lam. Hab.: Ceylon, Ettangwelle and Halgodde Pass, Matelle East,-Thwaites. Nilghiris, Animalayas; Coimbatore hills, on trees in moist woods. Beddome.

P. (Phyn.) hemionitideum, Wrall. Hab. : Nilghiris; Sampajee ghât (Kurg), four miles down from Mercara, in ravines, growing on rocks. Beddome.

P. (Phym.) pteropus, Blume. (P. tridactylon, Wall.). Hab.: Ceylon, Nluruta; Ambagamowa district, at no great elevation. - Thwaites. Animalayas, growing on rocks under water; very abundant in rivers; Nilghiris.

P. (Phym.) trifdum, Don. (P.oxylobum, Wall.). Hab.: Ceylon, more elevated parts of the Central Province. Nilghiris; on trees about Neddiwattan. Animalayas, 4,000 feet and upwards.

P. (Phym.) phymatodes, Linn. Hab.: Ceylon, common in the warmer parts of the island. Malabar, at no great elevation.

P. (Phym.) nigrescens, Blume. Hab.: Ceylon, common in forests of the Central Province. Thwaites; Hook. Fil. Ezot. t. 22.

P. (Phym.) longissimum, Blume. Hab. . Nilghiris; Bolamputty valley, in the Coimbatore hills; banks of the Pámbár. Foot of Carcoor ghât. Wynad. Beddome.

P. (Drynaria) quercifolium, Lim. Hab. : Ceylon, verv abundant upon old trees in the warmer parts of the island.-Thwaites. Very commion on the western side of the Madras Presidency, from the plains up to 4,000 feet, growing on trees and rocks. Beddome.

P. (Dry.) Linnæi, Bory. Hab. : Ceylon.

P. (Phym.) leiorhizon, Trall. Hab.: Animalayas, on the Kooch Mallay, 4,300 feet elevation. Beddome.

\section{Monogramme.}

M. Junghuhnii, Hook. Hab. . Ceylon, Central Proviuce, at an elevation of $3-5,000$ feet; not very common. Thwaites.

\section{Gymnogramme.}

G. (Leptogramme) Totta, Schlecht. (G. Lowei, Hook. \& Gr. Ic. Fil. t. 89). Hab.: Ceylon; common in the more elevated parts of the Central Province. Nilghiris; very common about Utacamund. Pulney hills.

G. (Stegnogramme) aspidioides, Hook. (non Kaulf.). Hab. : Ceylon.

G. Javanica, Blume. (G. serrulata, Blume.). Hab. : Ceylon, Horton Plains, and Maturatte district, at an elevation of 4-5,000 feet. Thwaites.

G. leptophylla, Desv. Hab. Nilghiris, on rocks between Utacamund and KKotaghire; Nahabaleshwar hills, Sattara Fort walls, Bombay. Beddome.

G. (Selliguea) lanceolata, Hook. Hab.: Ceylon; Pedro talagalla, at an eleration of 7,000 feet.-Thwaites. Nilghiris; Neddiwattan, on trees. Beddome.

G. (Sell.) involuta, Don. Hab.: Ceylon; Horton Plains, alt. 8,000 feet; common upon the trunks of trees in the Central Province. - Thwaites. Nilghiris, very abundant on the higher ranges, on rocks and trees. 
Tenisclam.

M. triphyllum, Swartz. Hab. : Ceylon, in the Central and Southern Provinces; Coimbatore hills, Bolamputty valley.

M. Thwaitesii, Hook. Hab. : Ceylon; Oodoo pussalawa, in the Central Province, at an elevation of 4,000 feet. $\$$.

\section{Antrophyum.}

A. plantagineum, Kaulf. Hab. : Ceylon, Central Province.

A. reticulatum, Kaulf. Hab. : Ceylon, very common in the upper forests of the Central Province; Nilghiris, in ravines on the Coonoor ghât; Animalayas, rather rare. Beddome.

A. semicostatum, Blume. Hab. : Ceylon.

\section{vittaria.}

V. elongata, Swartz. Hab.: Ceylon, Central Province; Animalaya hills, on trunks of trees in the teak forests from 2-3,000 feet elevation; Nilghiri slopes, between Neddiwattan and Coonoor; a common fern.

V. (Tæniopsis) falcata, Kunze. Hab. : Ceylon; Central Provinces, elevation $4-5,000$ feet.

V. (Tæniop.) linenta, Swartz. Hab. : Ceylon, common in the Central Province. Nilghiri slopes below Neddiwattan.

V. (Tæniop.) scolopendrina, Thw. (V. Zeylanica, Fée.). Hab.: Ceylon, forests of the Central Province; Nilghiris.

\section{Drymoglossam.}

D. piloselloides, Presl. Hab.: Ceylon, common on the trunks of trees; Nilghiris, Animalayas, Malabar. Hook. Gard. Ferns, t. 46.

\section{Femionitis.}

H. cordata, Roxb. Hab.: Ceylon, common in the warmer parts of the island; common in the dry localities of the Madras Presidency; seldom found at any great elevation.-Beddome. (H. cordifolia.) Hook. Fil. Exot. t. 35.

\section{Acrostichum.}

A. conforme, Swartz (A. laurifolium, Thonars.). Hab.: Ceylon, common in forests of the Central Province; Nilghiris; Animalayas, higher range, on trees.

A. viscosum, Swartz. Hab.: Ceylon, Nilghiris, Animalayas; rocks in the bed of the Toracadoo river, 4,000 feet elevation.

A. spathulatum, Bory. (A. piloselloides, Presl.). Hab.: Ceylon, Newera Ellia. Hook. Fil. Exot. t. 29.

A. squamosum, Swartz (A. paleaceum, Hook. \& Gr.). Hab. : Ceylon; Central Province, not very common.-Thwaites. Nilghiris, on trees about Neddiwattan, and on the Sisparah ghât; abundant. Beddome; Hook. \& Gr. Ic. Fil. t. 235.

A. (Stenochlæna) scandens, J. Sm. Hab.: Ceylon, common in the warmer parts of the island; Malabar; Animalayas, moist forests up to 4,000 feet.

A. (Egenolfia) appendiculatum, Willd. Hab. : Coylon, Nilghiris; Animalayas, moist forests, very common.

A. (Gymnopteris) variabile, Hook. Hab.: Ceylon.

A. (Gym.) quercifolium, Retz. Hab.: Ceylon, shady banks in the warmer parts of the island; Madras Peninsula, Animalayas; Travancore hills, rare. Hook. Fil. Exot.t. 80 ; Hook. Second Cent. Ferns, t. 5 . 
Acrostichum-continued.

A. (Gym.) Harlandii, Hook. (A. decurrens, Hook. Fil. Exot. t. 94.) Hab.: Ceylon, very abundant in damp forests. Thwaites.

A. (Gym.) repandum, Blume. Hab. : Ceylon, forests of the Central Province, common.-Thwaites. Common on western side of the Madras Presidency, in moist forests, at no great elevation; Animalayas.

A. (Gym.) Preslianum, Hook. Hab. : Concan.

A. (Gym.) virens, Wall. Hab.: Ceylon, in the forests of the Central Province; moist forests on the western side pof the Madras Presidency; Bolamputty valley, near Coimbatore; very abundant throughout Kurg. Hook. Second Cent. Ferns, t. 88.

A. (Chrysodium) lanceolatum, Hook. Hab.: Ceylon, very common in all alpine and subalpine forests on the western side of the Madras Presidency, growing on rocks and trunks of trees. Beddome.

A. (Chrys.) axillare, Cav. Hab.: Ceylon, Nilghiris.

A. (Chrys.) semicordatum, Buker. Hab. . Nilghiris, Concan. Hook. Second Cent. Ferns, t. 88.

A. (Chrys.) aureum, Linn. Hab. Ceylon, common in wet places, in the hotter parts of the island; Madras, banks of rivers and backwaters on the western coast, very abundant. Beddone.

A. (Hymenolepis) spicatum, Linn. Hab. . Ceylon, forests of the Central Province, not uncommon.-Thwaites. Paulghât mountains. Beddome; Hook. Gard. Furns, t. 3.

\section{osmunda.}

O. Javanica, Blume. Hab.: Ceylon, common in the higher parts of the Central Province; western ghât forests of the Madras Presidency.

O. regalis, Linn. Hab.: Bombay; abundant on the banks of rivers and streams on the Nilghiris, and other high mountains on the western side of the Madras Presidency.

\section{Schizea.}

S. dichotoma, Swz. Hab. : Nilghiris, Paughât hills.

S. (Actinostachys) digitata, Swz. Hab.: Ceylon, Kittool Galle, and near Peradenia. - Thwaites. Tranquebar. Hook. Gard. Ferns, t. 49 .

\section{Anemia.}

A. tomentosa, Suz. (A. Wightiana, Gardn.; A. flexuosa, Suz.). Hab. . Nilghiris, Sisparah ghât; dry places, 3-4,500 feet eleration. Animalayas; dry rocky places. Bieddome; Hook. Fil. Erot. t. 30.

\section{Iygodium.}

L. dichotomum, Suz. (L. circinnatum, Sir $)$.). Hab. . Ceylon, not uncommon in the Central I'rovince.-Therites. Common in most subalpine jungles on western side of the Madras Presidency. Malabar Plains, North Arcot Hills. Beddome.

L. scandens, Swz. Hab.: Ceylon, very common in the warmer parts of the island.-Thwaites. "Wyuar, very abundant.

L. pinnatifidum, Swz. Hab. . Ceylon, South India.

L. japonjeum, Swz. Hab.: Ceylon, common in the Central Province. Malabar. 


\section{Angiopteris.}

A. evecta, Hoffm. Hab. : Ceylon, common up to an elevation of 5,000 feet.- Thwaites. Very commou in most subalpine jungles on the western side of the Madras Presidency up to 4,000 or 5,000 feet. Beddome; Hook. Fil. Exot. t. 75.

\section{Marattia.}

II. fraxinea, Smith (M. sorbifolia, Sizz.). Hab.: Ceylon, more elevated parts of the Central Province.-Thwaites. Sisparah ghât, Bolamputty valley, Coinbatore hills. Beddome.

\section{Ophioglossum.}

O. nudicaule, $L$. ( $f l l$. .). Hab.: Animalayas; in wet grassy places, 2,500 feet elevation. Beddome.

O. reticulatum, Limn. Hab.: Animalayas, teak forests, Nilghiris. Beddome.

O. vulgatum, Linn. (O. ovatum, $\left.\Pi^{\top} i l l d.\right)$. Hab.: Ceylon, more elevated parts of the Central Province.-Thwaites. Animalayas, in swampy places, 2,500 feet elevation. Thwaites.

o. (Ophioderma) pendulum, Linn. Hab.: Ceylon, common upon the trunks of trees in the Central Province. Thwaites; Hook. Gard. Ferns, t. 33.

\section{Helminthostachys.}

II. zeylunica, Hook. Hab.: Ceylon, south of the island, on the borders of paddy fields, \&c.; not uncommon.-Thwaites. Animalayas; moist bamboo clumps in the teal forests, $2-3,000$ feet elevation. Malabar plains, foot of the Carcoor pass; very abundant.-Beddome. Cochin. Ilook. Gard. Ferns, t. 28; Second Cent. Ferns, t. 94.

\section{Botrychium.}

B. daucifolium, Nall. Hab. : Nilghiris, Animalayas; Bolamputty valley, 3,000 feet elevation; Ceylon, forests of the Central Province, up to an elevation of 4,000 feet. Thwaites.

B. virginianum, Suz. Hab.: Ceylon; Newera Ellia, on the turf under the shade of trees. - Thwaites. On most mountainous tracts of the western side of the Madras Presidency. Beddome; Hook. Gard. Ferns, t. 29. 


\section{EASTERN PENINSULA, INDTAN ARCHIPELAGO, PHILIPPINES.}

\section{Gleleheria.}

G. circinata, Swartz (Gl. Speluncæ, Br.; G. semivestita, Lab.). Hab. : Malacca.

G. dicarpa, $B r$. (Gl. alpina, Br.). Hab. : lofty burning mountain in Java, Celebes, Malacca, Mount Ophir. Hooki. Fil. Exot. t. 40.

G. (Mertensia) longissima, Bl. Hab. : Malay Islands; Borneo, elevation, 7,000 feet. Java, moist woods, Philippine Isles, Penang.

G. (Mert.) flagellaris, Spreng. Hab.: Java and Malaya, abundant. $\$$.

G. (Mert.) hirta, Bl. Hab. : Woody mountains of the Moluccas.

G. (Mert.) vestita, Bl. Hab. : Malay Islands. \$

G. (Mert.) dichotoma, Willd. Hab.: East Indies and Malay Isles, frequent. Tenasserim, Penang Singapore, Malacca, Java, Moluceas, Amboyna, Philippines.

\section{Cyathea.}

C. Brunonis, Wrall. Hab.: Pulo Penang, Malacca, Moluccas Isles, Borneo. \$.

C. crenulata, $B l$. Hab. : moist mountain woods, Java. \$\$.

C Javanica, Bl. Hab.: woods on the mountains of Java and Sumatra. \$\$.

C. integra, J. Sm. Hab. : Amboyna, Luzon, Pbilippine Isles. \$.

C. Sarawakensis, Hook. Hab. : Sarawak, Borneo. \$.

C. assimilis, Hook. Hab. : Hills, Sarawak, Borneo. \$.

C. Lobbiana, Hook. Hab. : Sarawak, Borneo. \$.

C. hymenodes, Mett. Hab. : Sumatra. \$\$.

C. orientalis, Moore. Hał.: Java. \$.

C. arachnoidea, Hook. Hab.: Island of Ternate. \$.

C. Korthalsii, Mett. Hab. : Sumatra, Java. \$.

C. dealbata, Swortz. Hab. : Penang.

C. aneitense, Hook. Hab.: Island of Ternate.

\section{zemitelia.}

H. Junghuniana, Mett. Hab.: Java, Sumatra.

\section{Alsophila.}

A. lunulata, Br.? Brack. Hab.: Java?

A. alternans, Hook. Hab. : Penang. \$

A.? Celebica, Mett. (Cyathea? Bl.). Hab.: Celebes and Ternate. $\$$.

A. comosa, Wall. Hab. : Singapore, Jara. \$8.

A. contaminans, Wall. (A. Brunoniana, $W^{-}$all.). Hab.. Penang, Java and Malay Islands; Luzon and S. Camarines. \$.

A. crinita, Hook. Hab. : Java.

A. tumentosa, Hook. Hab. : Java, S. Camarines, Formosa.

A. caudata, $J$. Sm. IIab. Luzon, Nanilla. $\$$.

A. ramisperia, Hook. Hab. : Surawak; Borneo, alt. 2,700 feet. \$.

A. squamulata, J. Sm. Hab.: Malacca, Mount Ophir, Philippines.

A. glabra, Hook. (A. gigantea, Wrall.). Ilab.: Java aud Malay Islands generally. Moulmein, Penang; mountrins of Tenasserim. 
Alsophila-continued.

A. latebrosa, Hook. Hab. : Malay Islands, Penang.

A. lurida, Hook. Hab. : Java and Celebes. \$.

A. crenulata, Mett. Hab. : Java. \$.

A. tristis, Bl. Hab. : Java. \$.

\section{Diacalpe.}

D. aspidioides, Bl. Hab. : Malay Islands; woods on the elevated mountains of Java.

aratonia.

M. pectinata, Br. Hab. : summit of Mount Ophir, Malacca. \$. D1cksonia.

D. (Cibotium) Barometz, Link. (C. glaucescens and C. Assamicum, Hook. Sp. Fil.). Hab. : Philippine Islands, Malayan Peninsula and Islands ; $\mathrm{S}$. China.

D. chrysotricha, Moore. Hab.: Java, ascending to 11,000
feet. $\$$.

D. sorbifolia, Smith. Hab.: Moluccas, Isle of Henimoe, Hindostan.

D. Moluccana, Blume. Hab.: mountains of the Moluccas, Java, \$.

1). Smithii, Hook. Hab. : Philippine Islands, Luzon, Manilla. \$.

\section{Iymenophyllum.}

H. parvifolium, Baker. Hab. : Moulmein. \$.

H. polyanthos, Suz. Hab. . Malayan Peninsula, Java, Luzon, Philippine Isles.

H. badium, Hook. \& Gr. Hab.: Malayan Peninsula. Hook. \& Gr. Ic. Fil. t. 76 .

H. Javanicum, Spreng. Hab.: the Malayan Peninsula, the Philippines. (H. crispatum, Hook. \& Gr. Ic. Fil. t. 77.) Java in mountain woods and in the Moluccas.

II. demissum, Swz. Hab.: Java, the Philippines.

H. flabellatum, Labill. Hab. : Philippines. J. D. Hooker.

$\mathrm{H}$. dilatatum, Sw. Hab. : rocks and trunks of trees, woods of Java. Hook. \& Gr. Ic. Fil. t. 60.

H. Zollingerianum, Kunze. Hab. : Java. \$.

H. Borneense, Hook. MSS. Hab. : Borneo; on hills near Sarawak, at an alt. of 2,700 feet. $\$$.

H. Smithii, Hook. Hab. : Philippine Islands, Java, and Malayan Peninsula; Burmah.

H. dichotomum, Cav. Hab. : high mountains of Java and the Moluccas.

H. denticulatum, Sw. Hab. : Moulmein, at 7,000 feet; and Java, on trunks of trees. $\$$.

H. Neesii, Hook. Hab. : Malayan Peninsula, Java, Borneo, and Philippines.

H. sabinæfolium, Baker. Hab.. Sahak, Java. \$.

\section{Trichomanes.}

T. Motleyi, V.D.B. Hab.: Borneo.

T. Henzaianum, Parish. Hab.: on trees; Moulmein, Pegu. Hook. Second Cent. Ferns, t. 1. \$.

T. muscoides, Swz. Hab. : Java.

T. parvulum, Poiret. Hab. : Java, Philippine Isles, Moluccas. 
Trichomanes-continued.

T. saxifragoides, Iresl. Iab. : Jara, Philippine Islands.

T. proliferum, Blume. Hab.: Java, Plilippine Islands.

T. digitatum, Swartz. Hab.: Java and Polynesian Islands. Hook. \& Gr. Ie. Fil. t. 33.

T. pusillum, Swartz. Hab. . Moulmein. Hook. \& Gr. Ic. Fil. t. 115 .

T. humile, Forster (Hook. \& G'r. Ic. Fit. t. 35). Hab. Java, Luzon.

T. pallidum, Bhome. (T. glauco-fuscum, Hook.; T. album, Bl.). Hab. : lofty mountains of Java, Sumatra, Malayan Peninsula, Penang, Philippine Islands.

T. serratulum, Baker. Hab. : on trees, Lobong Peak, Borneo. \$.

T. Filicula, Bory. Hab. : Java, Borneo.

T. auriculatum, Blume. Hab. . mountain rocks in Java, Philippines, Luzon.

T. denticulatum, Batier. Hab.: Borneo. \$.

T. Javanicum, Bl. (ITook. \& C'r. Yc. Fil. t. 240). Hab. : Birmab, shady places in Java, Singapore ; Penang, Malay Isles, Philippine Islauds, Borneo, Labuan, and Sarawak. Hook. Gard. Ferns, t. 37 .

T. brevipes, Baker. Hab. : Leyte, Pbilippine Islands. \$.

T. Smithii, IIook. Hab. : Philippine Islands. \$8.

T. rigidum, Swz. (T. striatum, Hook. \& Gr. Ic. Fil. t. 122). Hab.: Malayan Peninsula, Singapore.

T. meifolium, Bory. Hab. : Java, Philippine Islands.

T. maximum, Blume. Hab. J Java, Borneo.

T. Leprieurii, Kunze. Hab.: Singapure, East Indies.-Wallich; Hook. Gard. Ferns, t. 11. Philippine Islands.

T. giganteum, Bory. Hab.: Mialayan Archipelago.

'I'. gemmatum, J. Snith (T. cellulosum, Sturm. Hook. Second Cent. Ferns, t. 63). Hab. Java, Philippines, and Malayan Peninsula.

T. longisetum, Bory. (T. Pluma, Hook. Cint. Ferns, t. 97). Hab.: Borneo, near Sarawak; Java.

\section{Davallia.}

D. beterophylla, Smith. Hab. : Malayan Peninsula and Islands, Java, Penang, Singapore, Borneo, Sumatra, Samar Isle. Hook. \& Gr. Ic. Fil. t. $2: 30$; Hook. Fil. Exot.t. 27.

D. angustata, Wall. Hab. Malayan Peninsula and Islands; trunks of trees, Singapore, Penang. \$s.

D. parallela, Wall. Hab. : Malayan Peninsula, Burmah, Singapore, Luzon, Moluceas.

D. sessilifolia, Blume. Hab. : on trees, mountains of Salak, Java; Celebes.

D. pedata, Smith. IIab.: Malayan Peninsula, Java, Borneo, Singapore. Hook. Gark. Ferns, t. 7.

D. alpina, IJlume. (D. Belangeri, Bory.). Hab.: Mergui and Ledong; trunks of trees, and summit of Nount Gedé; Java, Borneo.

D. vestita, Blume. Hab. . trunks of trees, mountains of Java.

D. Cumingii, Hook. Hab.: Isle of Samar, Philippine Islands. \$.

D. Parishii, Hook. MSS. Hab. . limestone rocks, Moulmein. \$. 
Davallia-continued.

D. immersa, Wallich. Hab.: Malayan Peninsula, Moulmein, and Java. Hook. Fit. Exot. t. 79.

D. falcinella, Presl. Hab. : Malay Islands, Philippine Islands. \$.

D. chærophylla, Wallich. Hab.: Malayan Peninsula.

D. parvula, Wallich. Hab.: Singapore and Borneo, Java. \$. Hook. \& Gr. Ic. Fit. t. 138.

D. aftinis, Hook. Mlab. . Java, Penang, Luzon.

D. nodosa, Hook. Hab.: woods of the lofty mountains of Java and Molucea; summit of the mountains Gede, Burangrang, and Patuha in Java. Malayan Peninsula.

D. hymenophylloides, Baker. Hab.: Java. \$.

D. pulchella, Hook. Hab. : Philippine Islands.

D. repens, Desv. (D. Boryana, Presl.). Hab.: Java, Sumatra, Philippine Islands.

D. triquetra, Baker. IIab. . Java.

D. Blumeana, Hook. Hab. : mountains of Java, and Philippine Islands. \$.

D. Emersoni, Hook. \& Gr. Hab.: Penang, Java, Borneo, and Philippine Islands. Hook. \& Gr. Ic. Fil, t. 105.

D. cuntigua, Swz. Hab. : Java, Philjppine Isles. Hook. \& Gr. Ic. Fil. t. 141 .

D. triphylla, Hook. Hab.: Singapore. \$. t. 37 .

D. pentaphylla, Blume. Hab.: woods of Java, Hook. Fit. Exot.

1). Lobbiana, Moore. Hab. : Borneo. \$.

D. decurrens, Hook. Hab.: Isle of Bohol, Philippine Islands. \$.

D. solida, Swz. Hab. . Malayan Peninsula and Islands, Moulmein, Java, Penang, Singapore, Luzon, Isle of Vanicoro. Hook. Fil. Exot. t. 57.

D. elegans, Swz. Hab. : Malayan Peninsula, Java, Borneo, Singapore, Penang, Mergui, Rangoon.

D. epiphylla, Blume. Hab.: Java and Malay Peninsula. \$.

D. divaricata, Blume. (D. polyantha, Hook.). Hab. mountain woods of Java, and Malayan Peninsula. Singapore.

D. Griffithiana, Hook. Hab.: Malayan Peninsula.

D. bullata, Wallich. Hab.: Java and Malayan Peninsula.

D. (Microlepia) pinnata, Cav. Hab. . Malayan Peninsula, Java, and Philippine Isles.

D. (Micro.) ciliata, Hook. Hab.: Philippine Islands. \$\$.

D. (Micro.) strigosa, Swz. (D. Khasyana, Hook.). Hab.: Java, Malayan Peninsula, Pbilippine Islands.

D. (Micro.) inæqualis, Kunze. Hab.: Mindoro Island, Philippines.

D. (Micro.) campyleura, Kunze. (D. Amboynensis, Hook.). Hab. : Moluccas, Amboyna.

D. (Micro.) hirta, Kaulf. Hab. : Malajan Islands.

D. (Micro.) Spelunce, Baker (D. polypodioides, Hook.). Hab. : Singapore, Penang, Martaban, Java, Amboyna. Isles,

\section{Iindsaya.}

L. adiantoides, $J$, Sm. Hab. : Malay Archipelago. \$. 
Lindsaya-continued.

L. ovata, J. Sin. Hab. : Luzon. \$\$.

L. concinna, $J$. Sm. : Hab.: Philippine Islands, Borneo. \$.

L. cultrata, Swartz. Hab. . Malayan Peninsulas and Islands; Mergui, Java, Philippines, Luzon. Hook. \& Gr. Ic. Fil. t. 144 ; Hook. Fil. Exot. t. 67.

L. pectinata, Blume. (L. oblongifolia, Rrinw.). Hab.: Malayan Peninsula and Islands; rockss and trees in the interior of Java.

L. scandens, Hook. Hab.: Malayan Peninsula, Philippine Islands, Luzon. \$.

L. trapeziformis, Dry. (L. quadrangularis, Raddli L. caudata, Hook.). Hab.: Malayan Peninsula and Islands, Mlergui ; Penang, on the trunks of trees.

L. Borneensis, Hook. MSS. Hab. . Forests of Borneo. \$. $\$$.

L. rigida, J. Sm. Hab.: Malayan Peninsula, on Mount Ophir.

L. (?) Parishii, Baker. Hab. . banks of Packchang river, the southern boundary of the province of Tenasserim. \$\$.

L. (Isoloma) Walleræ, Hook. Hab.: Island of Banca, east of Sumatra.

L. (Iso.) divergens, Wall. Hab.: Malayan Peninsula, and Borneo. \$.

L. (Iso.) lanuginosa, Wall. Hab.: Malayan Peninsula; growing on trees in Singapore, Penang, Purmah.

L. (Iso.) retusa, Metten. Hab.: Philippines, Amboyna.

L. (Synophlebium) nitens, Bheme. (L. recurvata, Wall.). Hab. . Java, Singapore, Malacca, Penang.

L. (Syn.) davallioides, Blmme. Hab. : Malayan Peninsula and Islands ; Singapore, mountains of Java. \$.

L. (Schizoloma) cordata, Gutud. Hab. : Malayan Peninsula, rare; Rawak in the Moluceas. \$s.

L. (Schiz.) Gueriniana, Gaud. Hab.. Moluccas, Rawalr, Island of Celebes. $\$$.

L. (Schiz.) ensifolia, Swartz. Hab. : Malayan Peninsula, Mergui, Malay Islands, Luzon, Java, Borneo, Singapore. Hook. Gard. Ferns, t. 62 .

L. (Schiz.) heterophylla, Dry. Hab.: Malayan Peninsula and Islands.

\section{Adiantum.}

A. Parishii, Hook. Hab. : Moulmein; Malayan Peninsula, on a limestone rock (called Twa-Kabin) 2,000 feet elevation.-Beddome; Hook. Fil. E.cot, t. 51.

A. lunulatum, Burm. Hab. : Malay Islands and Peninsula ; Java, Philippines, Moluccas.

A. caudatum, Linn. (A. soboliferum, Wall.). Hab. . Malay Isles, Birmah, Java, Philippines.

A. diaphanum, Blume. (A. setulosum, J. Sm.). Hab.: near Lingain Jattie, Province of Cheribon, Java; Luzon.

A. pulchellum, Blume. (A. Lobbianum, Hook.). Hab.: Java. \$.

A. Athiopicum, L. (A. marginatum, Bory.). Hab.: Malacea.

A. flabellulatum, L. Hab. : Malayan Peninsula and Islands.

.Hypolepis.

H. tenuifolia, Bernh. Hab. : Java, Luzon. 


\section{Chellanthes.}

Ch. pteroides, Swartz. IIab. . summit of Mount Gede, Java.

Ch. fragilis, Hook. Hab.: limestone rocks near Moulmein, Malayan Peninsula. Hook. Fil. Exot. t. 96.

Ch. varians, Hook. Hab.: Malayan Peninsula, Luzon.

Ch. multifida, Swartz. Hab. : lofty mountains of Java.

Oh. tenuifolia, Swartz. Hab.: Malayan Peninsula and Islands, frequent; Moulmein, Tavoy, Philippine Islands.

Ch. rufa, Don. Hab.: Burmah; Mergui, in Tenasserim.

Ch. argentea, Hook. Hab.: Moulmein (on the same rock with Adiantum Parishii). Hoo\%. Fil. Exot. t. 95.

Ch. farinusa, Kaulf. Hab. : Malayan Peninsula, Java, Philippine Islands.

\section{Onychium.}

O. auratum, Kaulf. Hab.: Malayan Peninsula and Islands; Manilla, Luzon, Java.

O. Japonicum, Kunze. (O. lucidum, Spreng.). Hab. : Java.

\section{Pellax.}

P. falcata, Fée. Hab. : Malayan Peninsula.

\section{Pteris.}

Pt. longifolia, L. Ilab. : Malay Archipelago, Borneo, Malacca, Singapore, Penang, Java, Amboyna, Luzon.

Pt. moluccana, Blume. Hab.: Malayan Islands. \$\$. $\$$.

Pt. opaca, J. Sm. Mab. : Island of Samar, one of the Philippines.

Pt. cretica, L. Hab.: Penang, Java, Philippines.

Pt. pellucida, Presl. Hab.: Malayan I'euinsula, Chittagong, Philippines, Irawaddy.

Pt. distans, J. Sm. Hab.: Luzon, Philippine Islands. \$\$.

Pt. heteromorpha, Fée. Hab.: Philippine Islands, Birmah, Luzun. $\$$.

Pt. semipinnata, Linn. Hab. . the Philippines and Borneo, Java. Hook. Giard. Ferns, t. 59.

Pt. Dalhousiæ, Hook. Hab. : Penang, Java. \$.

Pt. quadriaurita, Retz. Hab. . Malay Isles, Java, Singapore, Penang ; South Ilicos, Philippine Islands; Chittagong.

Pt. longipinnula, Wallich. Hab.: Malayan Peninsula, and Borneo.

Pt. excelsa, Gaud. Hab. mountains of Ava; mountains near Baños, Luzon.

Pt. aquilina, L. Hab. : Singapore, Penang, Borneo.

Pt. biaurita, $L$. Hab.: Ava, Penang, Java.

Pt. patens, Hook. Hab.: Borneo, Philippines.

Pt. Wallichiana, Agwrlh. Hab.: Japan and Philippine Islands.

Pt. ludens, Wall. Hab. : Malayan Peninsula, Irawaddy, and cares of the mountain Nidan; Attran River, Moulmein, Luzon. \$.

Pt. Junghunii, Baker. Hab. : Java. \$.

I't. incisa, Thunb. (Pt. aurita, Bl.). Hab. : Java, Luzon, Borneo, Moulmein.

Pt. tripartita, Suz. Hab. : Java, Molucca, Amboyna, Labuan, Luzon, Singapore. 


\section{Ceratopteris.}

C. thalictroides, Brong. Hab. : throughout the tropics in quiet waters. Singapore, Penang, Java, Luzon; Isle of Negros (Philippines), east coast of Timor.

\section{Iomaria.}

L. Patersoni, Spreng. (L. elongata, Blume.). Hab. : Luzon.

L. vulcanica, Blume. Hab. : Java, Mount Gedei. Hook. Cent. Ferns, t. 59.

L. punctulata, Spreng. Hab.: lofty mountains of Java.

L. procera, Spreng. Hab. : Malay Islands, lofty mountains of Java, and summit of Mount Tjerimai ; province of Cheribon, and summit of lofty mountain Ternates, Luzon.

L. adnata, Blume. Hab.: Java.

L. glauca, Blume. Hab. : Java.

L. pycnophylla, Kunze. Hab. . Jara, Malayan Peninsula.

\section{Blechnum.}

B. nitidum, Presl. Hab. : Isle of Sorzogon, Luzon.

B. serrulatum, Rich. (B. striatum, Br.). Hab.: Malacca ; moist open places, Tuabuan.

B. orientale, Lim. Hab.: Malay Islands, Luzon, Java, Amboyna, Borneo; Moulmein, Penang, Singapore, Amherst. Hook. E. ot. Fil. t. 77.

B. Finlaysonianum, Irall. Hab. : Penang, Singapore, Labuan, Borneo. Hook. S, Gi, Ic. Fil. t. 225. \$.

\section{Sadleria.}

S. cyatheoides, Kaulf. Hab.: Sumatra.

\section{woodwardia.}

W. radicans, Smith. Hab.: Java.

\section{Asplenium.}

A. Nidus, L. (A. m"sæfolium, Mett.). Hab.: Penang and Rangoon, Singapore; Malay Islands, abundant; Java, Borneo, Luzon.

A. Grevillei, Wall. Hab.: Birmah, Tavoy. Hook. \& Gr. Ic. Fil. t. 128 . \$ $\$$

A. ensiforme, Trall. Hab.: Moulmein. Hook, \& Gr. Ic. Fil. t. 71 .

A. concolor, Hook. Hab. : Java. \$\$.

A. squamulatum, Blume, Hab.: on limestone rocks, Bormeo, Sarawak, Banjermassing, Java. \$.

A. scolopendrioides, J. Sm. Mab. . Island of Leyte, Philippines. Dahumkung River; Kina Balu, elevation, 3,000 feet; Borneo. Hook. Cent. Ferns, t. 30.

A. Sundense, Blume. (A. rittæforme, Cav.). Hab.: Jara mountain woods; Isle of Leyte, Philippine Islands. $\$$.

A. Griffithianum, Hook. Hab.: Mishmee.

A. subavenium, Hook. Hab.: Penang. \$.

A. longissimum, Blume. Hab. . Java, Singapore, Burnah, Mergui, Malacca, Borneo, Sumatra.

A. Sumatranum, Hook. Hab.: Sumatra. $\$$.

A. salignum, Blume. Hab. : shady mountain woods, Java. \$.

A. rulcanicum, Blume. (A. heterodon, Mett.). Ilab. . Jara, parasitic on trees. 
Asplenium-continued.

A. tenerum, Forst. (A. elongatum, Sw.). Hab. : Luzon, Java, Singapore, Penang, S. Borneo; W. Sumatra.

A. erectum, Bory. (A. Brasiliense, Raddi). Hab. : East Indies, Malayan Peninsula.

A. persicifolium, J. Sm. Hab.: Luzon, Isle of Bohol, Philippines.

A. fuliginosum, Hook. (A. lugubre, Hook. Second Cent. Ferns, t. 3). Hab. . Kina Balu, Borneo. \$\$.

A. Borneense, Hook. Hab.: Borneo; banks of the Dahumbuy River, elevation 2,000 feet abore the sea. \$.

A. anisodontum, Presl. Hab.: Java and Philippines. \$.

A. contiguum, Kaulf. Hab.: Philippines, Luzon.

A. hirtum, Kaulf. Hab. : Philippines, Java, Penang, Borneo, Sarawak; Banjarmassing, S. Borneo.

A. falcatum, Lam. Hab.: Moulmein, on trees; Taroy.

A. caudatum, Forst. Hab. : Malay Isles, Java, Luzon.

A. macrophyllum, Swartz. Hab. : Malayan Peninsula and Islands ; Java, Singapore, Penang, Malacca, Luzon; Mishmee and Tavoy; Borneo, Sarnwak, Banjarmassing; Sumatra.

A. paradoxum, Blume. (A. zamioides, Hook.). Hab. : Java, Sumatra, Penang. \$.

A. resectum, Simith. Hab.: Moulmein, Java, Sumatra, Chittagong.

A. heterocarpum, Wall. Hab.: Malayan Peninsula, Borneo, Moulmein.

A. planicaule, Wall. Hab. . Mishmee.

A. horridum, Kaulf. Hab. : Java.

A. Adiantum-nigrum, Linn. Hab. : Java.

A. cuneatum, Tam. Hab. Java, Sumatra, Borneo.

A. furcatum, Thunb. Hab. : Mergui, Java.

A. affine, Swartz (A. spathulinum, J. Sm.). Hab.: Luzon; Borneo, at the Lobong river.

A. nitidum, Swartz. Hab. : Malacca, Java, Labuan, Singapore, Moulmein, S. Borneo.

A. laserpitiifolium, Lam. Hab. : Malay Islands, Java, Luzon.

A. bulbiferum, Forst. Hab. . Penang.

A. tenuifolium, Don. (A. concinnum, Tall.). Hab.: Malayan Peninsula.

A. (Darea) dichotomum, Hook. Hab. . Borneo; Lobouk Peak, eleration 5,000 feet, north-east side of Borneo. Hook. Second Cent. Ferns, t. 39 . \$.

A. (Darea) Belangeri, Kunze. Hab.: Malayan Peninsula, Java, Sumatra, Borneo.

A. (Darea) scandens, J. Sm. Hab.: Philippine Isles, Leyte. Hook. Second C'nt. Ferns, t. 37.

A. (Athyr.) grammitoides, Hook. Hab.: Peninsular India, Java, Philippines, Luzon. Hook. ('ent. Ferns, t. 13.

A. (Athyr.) thelypteroides, Michx. Hab. : Penang.

A. (Athyr.) deparioides, Bracli. Hab. : Sumatra.

A. (Athyr.) macrocarpum, Blume. Hab. : Java.

A. (Athyr.) nigripes, Blume. Hab.: Java.

A. (A thyr.) oxyphyllum, Hook. Hab. : Java. 
Asplenium-continued.

A. (Athyr.) brevisorum, Wall. Hab.: Toong Dong, mountaing of Ava. Mishmee.

A. (Athyr.) umbrosum, J. Sm. (A. australe, Brack.). Hab.: Java,

A. (Athyr.) Woodwardioides, Baker. Hab.: Philippines, Java. \$\$.

A. (Dipl.) subserratum, Blume. Hab. : Java and Penang. $\$$.

A. (Dipl.) pallidum, Blume. Hab.: Philippines, Java, and Malayan Peninsula and Isles; Moulmein. S. Camarines. \$\$.

A. (Dipl.) porrectum, Ifall. Hab.: Malayan Peninsula and Isles; Chapadong Hill, Penang, Singapore, Borneo. S.

A. (Tipl.) cultratum, Mett. Hab. : Luzon. \$

A. (Dipl.) bantamense, Baker. (A. fraxinifolinm, Wall.; A. glternifolium, Mett.). Hab.: Halaccas, Borneo, Java, Penang, Singapore. Hook. Second Cent. Ferns, t. 19; Hook. Fil. Evot. t. 17 .

A. (Dipl.) Lobbianum, Huok. Hab.: Java. Hook. Second Cent. Ferns, t. 17.

A. (Dipl.) sylvaticum, Presl. Hab. : Malayan Peninsula, Java, Borneo, Penang, Singapore.

A. (Dipl.) Brackenridgii, Baker. Hab. : Pbilippines.

A. (Iipl.) tomentosum, Hook. (non Mett.). Hab.: Burmah; Mergui, in damp, dark, cavernous places, on banks near watercourses. Malacca, Java, Sumatra.

A. (Dipl.) lasiopteris, Mett. Hab.: Java.

A. (1)ipl.) speciosum, Mett. Hab.: Malayan Peninsula and Philippines, Java.

A. (Dipl.) Sorzogonense, Presl. Hab. : Luzon and Sorzogan, Java, Leyte; foot of Moohoo mountain, Borneo ; Sunatra.

A. (Dipl.) deltoideum, Presl. Hab. : Luzon. \$.

A. (Dipl.) cyathæfolium, Bory. Hab. : Luzon.

A. (Dipl.) Mejenianum, Mett. Hab. : Manila. \$.

A. (Dipl.) polypodioides, Mett. Hab. : Malayan Peninsula and Isles; Java, Singapore, Luzon, Mishmee, Tonghoo, Joulmein.

A. (Dipl.) vestitum, Presl. Hab.: Isle of Samar, Philippines. Flook. Serourd Cent. Ferns, t. 46 . \$\$.

A. (Dipl.) latifolium, Don. Hab. : Philippines.

A. (Aviso.) cordifolium, Mett. Hab.: MLalayan Peninsula and Isles, Philippines, Java, Singapore, Penang, Isle of Leyte. Hook. C'ent. Ferns, t. 36.

A. (Aniso.) alismæfolium, Hook. Hab. : Luzon, Sorzogon. \$\$.

A. (Aniso.) lineolatum, Mett. IIab.: Philippine Islands, and Malayan Peninsula and Islands; Java, Luzon, Borneo, Labuan. \$. Hook. C'ent. Ferns, t. 39-10.

A. (Aniso.) decussatum, Sicurtz. Hab. Malayan Islands, Java, Isle of Leyte, Ternate, Moluccas, Borneo.

A. (Aniso.) esculentum, Presl. THab.: common, apparently, all over the continent of India. Malayan Pexinsula and Isles; Java, W. Sumatra, Borneo, Celebes.

A. (Hemi.) Finlaysonianum, Thall. Hab. : Penang and the neighbouring islands. 


\section{Allantodia.}

A. Brunoniana, $\mathrm{TI}^{\top}$ ill. Hab. : Java.

Actiniopteris.

A. radiata, Link. Hab. : Sikaan in Ava.

\section{scolopendrium.}

S. pinnatum, J. Sm. Hab. : Philippines. \$.

\section{Didymochlsena.}

D. lunulata, Desv. Hab.: East Indies, Java, Luzon, Malayan Peninsula. Hook. Gard. Ferns, t. 17.

D. polycarpa, Baker (Nephrodium Jayanicum, Hook.). Hab. : Malayan Peninsula and Islands; Java, Singapore, and Penang; Island of Nusa Kambagan, Moluccrs, Hook. Fil. Exot. t. 62. \$.

\section{Aspidium.}

A. (Polyst.) semicordatum, Sw. Hab.: Luzon, Borneo, Twa Kabin, Moulmein, Cochin China.

A. (Polyst.) aculeatum, Sw. Hab. J Java, Moluccas, Singapore, Moulmein.

A. (Polyst.) obtusum, Mett. Hab. : Luzon. \$.

A. (Polyst.) amabile, Blume. Hab. : Malacca.

A. (Polyst.) aristatum, Sw. Hab. . Java, Borneo, Luzon, Moulmein.

A. (Cyclo.) Hookeri, ,Baker. Hab.: Malay Archipelago. (A. nephrodioides.) Hook. $\$$.

A. angulatum, J. Sm. (non Hook.). Hab.: Malay Archipelago, Java, Amboyna, Borneo. \$.

A. platanifolium, Mett. Hab.: Malayan Isles. $\$$.

A. repandum, Willd. Hab. . Philippines. $\$$.

A. calcareum, Presl. Hab. : Philippines, Isle of Leyte. $\$$.

A. membranaceum, Hook. Hab. : Java, Philippines.

\section{Nephrodium.}

N. (Lastrea) hirtipes, Hook. Hab.: Malayan Peninsula and Isles; Moulmein, Java.

N. (Last.) gracilescens, Hook. Hab. : Java.

N. (Last.) patens, Desv. Hab. : Philippines, Samar.

N. (Last.) attenuatum, Baker. Hab. : Philippines. \$.

N. (Last.) immersum, Hook. Hab.: Philippines, Malacca, Sumatra, Borneo.

N. (Last.) ligulatum, Hook. Hab. : Philippines, Isle of Samar. $\$$.

N. (Last.) calcaratum, Hook. ( $N$. falcilobum). Hab. : Malay Islands, Java, W. Sumatra, and Philippines; Moulmein.

N. (Last.) viscosum, Baker. Hab.: Malacca, Philippines, and Borneo. \$.

N. (Last.) crassifolium, Hook. Hab.: Philippines and Malacca; Java, Luzon, W. Sumatra, S. Borneo, Malay Peninsula, Penang. \$\$.

N. (Last.) echinatum, Baker. Hab. : Borneo. \$8.

N. (Last.) exiguum, IIook. Hab.: Philippines. \$.

N. (Last.) sagenioides, Baker. (N. melanopus, Hook.). Hab.: Malayan Peninsula and Islands; Moulmein, Amboyna. \$

N. (Last.) syrmaticum, Baker (N. spectabile, Hook.). Hab.: Java, Luzon, Moulmein, Malay Peninsula.

N. (Last.) Filix-mas, Rich. IIab. : Malay Islands and Peninsula; Jara, Penang. Hook. Fil. Eirot. t. 98. 
Tephrodium-continued.

N. (Last.) Preslii, Baker. Hab. : Philippines. \$.

N. (Last.) Rorneense, Hook. Hab. : Sarawak ; Borneo, on limestone rocks. Hook. Cunt. Ferns, t. 93. \$.

N. (Last.) flaccidum, Hook. Hab.: Jara and Mnulmein.

N. (Last.) setosum, Baker. Hab.: Java ; Moulmein, 6,000 feet elevation. \$8. Java.

N. (Last.) sparsum, Don. (N. purpurascens, Hook.). Hab.:

N. (Last.) edentulum, Baker. Hab. Java. \$\$.

N. (Last.) odoratum, Baker (N. eriocarpum, Dec.). Hab.: Malayan Peninsula, Irawaddy, Moulmein.

N. (Last.) Parishii, Hook. Hab. . Moulmein, shady moist places, limestone rocks. $\$$.

N. (Last.) recedens, Hook. Hab. : Philippine Islands, Java.

x. (Last.) membranifolium, Presl. Hab.: Malay Peninsula and Archipelago; Tenasserim, Moulmein, Malay Islands.

N. (Last.) spleudens, Hook. Hab.: Malaccas, Malay Peninsula.

N. (Last.) iutermedium, Baker. Hab.: Philippines, Malaccas. Java.

N. (Last.) Boryanum, Baker (non IIool.). Hab.: Moulmein,

N. (Last.) setigerum, Baker (N. tenericaule, Hook.). Hab.: Java, Borneo, Philippine Isles.

N. (Last.) platypus, Hook. Hab.: mountains; Moulmein, alt. 5,000 feet. Java.

N. otaria. Luker (N. aristatum, Hook.). Hab.: Philippines.

N. unitam, R. Br. (non Sieb.; N. propinquum, Br.). Hab.: Malay Peninsula and Islands, Singapore, Moluccas, Java, Borneo.

N. pteroides, $J$. Sm. Hab.. Malaccas, Philippines.

N. extensum, Hook. Hah.: Malaccas, Philippines.

N. procurrens, Baker. Hab. : Java. \$\$.

N. Longipes, Hoore. Hab. : Jara. \$.

N. cucullatum, Baker. IIab. Mrilaccas. (N. unitum, Sieb.) Java, Borneo.

N. Haenkeanum, Presl. Hab. : Malaya.

N. multijugum, Brtirr. Hab.: Malayan Peninsula. \$.

N. hirsutum, J. s'm. Hah.: Philippines, Luzon.

N. aridum, Bulirr. Hab. . Philippines, Malay Peninsula.

x. olandulosum, $J$. Sm. Hab.: Philippines, Malay Islands, Jara, Island of Leyte.

N.. Amboinense, Presl. Hah.: Philippines. \$.

N. Arbuscula, Desv. (N. Ilookeri, $\mathbb{W}^{\prime}$ all. ). Hab. : Amboyna, Philippines. Hot, Cent. Fern, t. 22.

N. abortivum, $J$. Sin. Tab.: Penang, Jara.

N. latipinna, Hook. 1lsis. Hab. : Java.

N. pennig rum, Hook. Hab. : Halaccas, Penang.

N. cyatheoides, Frult. Halo. Sumatra.

X. hispidulum, Later (N. angustifolium, Presl.). Hab. Philippines, Amboyna, Timor, Malaya. \$.

N. heterocarpon, IHowre. IIab. : Malaccas, Java, Borneo.

N. stipellatum, Hook. Ilab.: Jara, Sumatra. \$

N. crinipes, Hook. Hab. : Malacca.

N. ferox, Musere Hab Philippined, Luzon, Jara. 
Nephrodium-continued.

N. sagittæelium, Moore. Hab, : Java. \$.

N. truncatum, Presl. Hab.: Malacca, Java, Sumatra, Borneo, Penang, Singapore.

N. (Pleo.) heterophyllum, Hook. Hab.: Philippines. Hook,

Cent. Ferns, t. 20.

N. (Pleo.) excellens, Bl. Hab.: Java. \$s.

N. (Pleo.) Leuzeanum, Hook. Hab.: Moluccas, Java, Ceram, Luzon.

N. (Sagen.) Singaporianum, Baker. Hab.: Malay Isles, Singapore, Penang. Hook. \& Gr. Ic. Fil. t. 26. \$.

N. (Sagen.) ternatum, Baker. Hab. : Borneo. \$.

N. (Sagen.) vastum, Baker (Aspid. alatum, Wall.). Hab.: Malacca, Singapore, Java, Borneo. Hook. \& Gr. Ic. Fil. t. 184.

N. (Sagen.) melanocaulon, Baker. Hab. : Philippines and Malay Isles; Java, Sumatra. \$.

N. (Sagen.) subtriphyllum, Baker (Aspid., Hook.). Hab.: Malaya, Moulmein.

N. (Sagen.) Lobbii, Baker (Aspid., Hook.). Hab.. Borneo, Sarawak. \$.

N. (Sagen.) semibipinnatum, Baker (Aspid., Hook.). Hab.: Penang and Borneo.

N. (Sagen.) Meyanthidis, Baker (Aspid., Pr.). Hab.: Philippines.

N. (Sagen.) irriguum, Baker (Polyp. menisciicarpon, Hook. in part.). Hab.: Philippines. \$s.

N. (Sagen.) polymorphum, Baker (Aspid., Trall.). Hab. : Malayan Peninsula and Isles; Moulmein, Borneo.

N. (Sagen.) Zollingerianum, Baker (Aspid. immersum, Hook.). Hab. : Java, Tenasserim. \$.

N. (Sagen.) pariolosum, Baker (Aspid., Wall.). Hab.: Moulmein, Mergui, Malaya, Amherst, Tavoy.

N. (Sagen.) decurrens, Baker (Aspid. pteropus, Kunze.). Hab. : Malay Isles, Borneo, Sarawak, Luzon.

N. (Sagen.) siifolium, Baker (Aspid. Teysmannianum, Hook.). Hab. : Java, Sumatra, Philippines. \$.

N. (Sagen.) pachyphyllum, Baker (Aspid. grande, J. Sin.). Hab. : Philippines and Malay Islands.

N. (Sagen.) cicutarium, Bulier (Aspid., Sw.). Hab.. Malay Islands, Java, Moulmein.

N. (Sarren.) Grittithii, Baker. Hab.: Burmah. \$s.

N. (Sagen.) giganteum, Baker (Aspid., Bl.). Hab.: Java, Isle of Leyte, Moulmein.

\section{Nephrolepis.}

N. exaltata, Schott. Hab.: Malay Archipelago, Java.

N. acuta, Presl. Hab.: Java, Borneo, Malacea, Moulmein,

N. ramosa, Baker (N. obliterata, Hook.). Hab. . Philippines, Malaceas, Java, Luzon.

N. floceigera, Moore. Hab. : Java and Celebes. \$.

N. darallioides, Kze. Hab. : Jara. Hook. Fil. Ertot. t. 60.

\section{oleandra.}

O. neriiformis, Cav. Hab.: Philippines and Malaccas. Hook. Fil. Exot. t. 58. 
Olecudra-continued.

O. musæefolia, Kunze. Hab.: Malay Islands.

O. Cumingii, J. Sm. IIab. : Philippines, Luzon, Burmab, Malaya.

\section{Polypodium.}

P. (Pheg.) erubescens, Wall. Hab. : Mrajayan Peninsula and Isles.

P. (Pheg.) appendiculatum, Trall. Ilab. : Penang:

P. (Pheg.) distans, Don. (P. paludosum, Bl.). Hab. : Java.

P. (Pheg.) obscurum, Hook. Hab.: Philippines, Leyte, Tavoy, Java. $\$$.

P. (Pheg.) Hasseltii, Blume. Hab.: Java. \$.

P. (Pheg.) rufescens, Llwme. Hab.: Java.

P. ('lheg.) asperulum, J. Sim. Hab.: Philippines. \$.

P. (Pheg.) punctatum, Thunl. (P. rugulosum, Labill.). Hab. : Philippines, Malaccas.

I. (Pheg.) Keraudrenianum, rand. Hab. : Java.

1'. (Goniopteris) simplicifolium, Hook. (Nephrodium, Hook. Second C'ut. Farns, t. 19). Hab. : Samar, Philippine Islands.

P. (Goniopt.) debile, Baker. Hab.: Amboynn. $\$$.

P. (Goniopt.) Borneense, Hook. Hab. : Borneo. \$.

P. (Goniopt.) rubidum, Hook. Hab.: Java, Philippines. \$.

P. (Goninpt.) urophyllum, I'all. Hab.: Moulmein, Rangoon, Penaug, Jara, Luzon, Malaccas.

P. (Goniopt.) proliferum, Presl. Hab. . Philippines, Java, Luzon.

P. (Goniopt.) lineatum, Colebr. Hab.: Penang, Mishmee, Malaccas.

P. (Dictyopteris) Barberi, Hook. Hab.: Malay Peninsula and Islands, Pulo-Penang, Borneo. $\$$.

P. (Dicty.) megalocarpum, Hook. Hab. : Java. $\$$.

P. (Dicty.) tenerifrons, Hook. Hab. . Moulmein, among limestone rocks. Luzon. Beddome. \$s.

P. (Dicty.) macrodon, Rcinw. (P. Cumingianum, Hook.). Hab. : Philippines, Malaccas.

P. (Dicty.) Brogniartii, Bory. Hab. : Malay Islands and Philippines; Sorzogon, Waigion, Luzon. \$\$.

P. (Dicty.) difforme, Blume. (P. irregulare, Pr.). Hab. . abundant in the Malay Islands, Borneo, Luzon, Malacca, Moulmein.

P. Hookeri, Brack. Hab. : Philippines.

P. subevenosum, Baker. Hab. . Penang. \$.

P. birtellum, $B l$. Hab. : Java. \&.

P'. hirtun, Hook. Hab.: Luzon, Java. \$.

P. setigerum, Blume. Hab. : epiphytal; on trunlss and branches of trees, Java. Hook. Cent. Ferns, t. 41 . \$.

P. (Gramnitis) bisulcatum, Hook. Hab.. Borneo, near Sarawak. Hook. Cent. Ferns, t. 98. \$8.

P. (Gram.) sessilifolium, Hook. Hab.: Philippines, Penang, Luzon, Java, Malaya. \$.

P. (Gram.) fasciatum, Mett. Hab.: Java; on mountains, alt. $3-5,000$ feet. $\$$.

P. Alabellivenium, Baker. Hab.: Borneo. \$.

T. subpinnatifidum, Bl. Hab.: Java.

P. cucullatum, Nees. Hab. : Java, Luzon, Mount Ophir, Malacca, Kina-balu, Borneo. 
Polypodirem-continued.

P. solidum, Mett. Hab. : Java. \$\$.

P. subserratum, Hook. IIab. : Borneo. \$.

P. trichomanoides, Swz. Hab.: Malacea.

P. sarmentosum, Brack. Hab.: Sumatra.

P. Adenophorus, Hook. \& Arn. Hab.: Sumatra.

P. obliquatum, Blume. Hab. : Java, Penang, Luzon, Celebes.

P. subsecundo-dissectum, Zolling. Hab.: Java. \$\$\$

P. minutum, Blume. Hab. : summit of Mount Gedé, Java, on the trunks of trees. Sumatra, Philippines, Luzon.

P. subfaleatum, Blume. Hab.: Nalay Isles, Java, Luzon.

P. Celebicum, Blume. IIab.: Borneo and Celebes; on trees in woody mountains of Celebes, Klobat. \$\$.

P. fuscatum, Blume. Hab.: on trees in mountain woods of Java.

P. decorum, Brack. Hab. : Malaya and Philippines, Singapore, Mount Ophir, Luzon, Borneo.

P. Lobbianum, Hook. Hab.: Borneo, Sarawal; ; on trees, alt. 2,500 feet. $\$$.

P. papillosum, Blume. Hab. Java, Sumatra, Philippines. Hook. Cent. Ferns, t. 46.

P. clavifer, Hook. Hab. : Kina-balu, Borneo. \$.

P. venulosum, Blume. Hab.: Malay Islands, Java.

P. hymenophylloides, Kaulf. (Adenophorus, Hook.\& Gr. Ic. Fil. t. 176). Hab. . Sumatra.

P. tenuisectum, Blume. Hab.: Java, in clefts of rocks, and on old trees on the lofty mountains. Hook. Second Cent. Ferns, t. 21. $\$$.

P. tamariscinum, Kaulf. Hab.: Malay Isles. (Adenophorus.) Hook. \& Gr. Ic. Fil. t. 175.

P. millefolium, Blume. Hab. : Java. \$.

P. dareæforme, Hook. Hab.: Moulmein. Hook. Second Cent. Ferns, t. 24.

P. subdigitatum, Bheme. (P. davallioides, Mett.). Hab. : Malay Peninsula and Islands; Java, Kina-balu ; Borneo, alt. 6,000 feet.

P. (Gonioph.) nummularium, Mett. Hab. : Philippines. $\$$.

P. (Gonioph.) persicæfolium, Desv. (P. cuspidatum, Blume.). Hab. : Java.

P. (Gonioph.) puberulum, Baker. Hab. : Burmah. \$.

P. (Gonioph.) subauriculatum, Blume. (P. argutum, Wall.). Hab.: Java, Sumatra, Philippines, Celebes.

P. (Gonioph.) verrucosum, Wall. Hab.: Penang and Singapore, Borneo, Sumatra, Amboyna, Ceram, Java, Luzon. Hook. Gard. Ferns, t. 41 . \$.

P. (Gonioph.) Korthalsii, Mett. Hab.: Sumatra. \$.

P. (Niphobolus) adnascens, Sw. Hab.: Moulmein, Malay Islands, Java, Celebes, Sumatra. Hook. Gard. Ferns, t. 19.

P. (Niph.) acrostichoides, Forst. Hab. : Java, Isle of Samar, Moluccas, Moulmein.

P. (Niph.) Lingua, Sw. Hab. . Malay Islands and Peninsula, Borneo, Luzon.

P. (Niph.) sphærostichum, Mett. Hab. : Luzon. \$.

P. (Niph.) stigmosum, Sw. (P. costatum, Wall.). Hab. : Malay

Peninsula and Islands; Moulmein, Java. 
Polypodium-continued.

P. (Niph.) nummulariæfolium, Mett. Hab.: Java, Luzon, Sunatra, Borneo, Celebes.

P. (Niph.) floccigerum, Mett. Hab.: Philippines, Malacca, Java, Sumatra.

P. (Niph.) fissum, Baker (P. porosum, $T V^{\top}$ all.). Hab.. Java.

P. (Niph.) albicans, MIett. Hab.: Java. \$.

P. (Niph.) distichocarpum, Mett. Hab. . Sumatra. \$.

P. (Niph.) Penangiamum, Hook. Hab.: Burmah, Penang. \$.

P. (Niph.) splendens, Hook. Hab.: Isle of Samar, Philippines. 8 .

P. (Phymatodes) subecostatum, Hook. Hab.: Bormeo, trees; Sariwak, on mountains 1,000 feet. $\$$.

P. (Phym.) accedens, Blume. Hab.: Malacca, Philippines, Luzon, Java, Sumatra.

P. (Phym.) oodes, Kunze. Hab. : Philippines, Luzon. \$.

P. (Phym.) lineare, Thunb. (P. loriforme, $T^{*}$ all.). Hab. : Malay Islands, Sumatra. Flook. Gard. Ferns, t. 14.

P. (Phym.) tenuilore, Kunze. Hab. . Philippines, Luzon, and Isle of Samar. \$\$.

P. (Phym.) stenophyllum, Bl. Hab.. Java, Luzon, Penang, Malacca, Mount Ophir. \$.

P. (Phym.) soridens, Hook. Hab. : Borneo; Sarawak, on trees. \$.

P. (Phym.) sinuosum, Wall. Hab.: Malaccas, Amboyna, Java, Mergui, Burmah.

P. (Phyn.) longifolium, Mett. Hab. : Malaccas and Philippines, Burmab, Mergui, Singapore, Java, Luzon. Hook. Second Cent. Ferres, t. 87 . \$s.

P. (Plym.) superficiale, Blume. Hab.: Java; Chappadong Nountain, Tenasserim.

P. (Phym.) angustaturn, Sw. Hab. . Singapore, Malacca, Penang, Borneo, Java. Hook. Gard. Ferns, t. 40.

P. (Phym.) Samarense, Mett. Hab.: Samar, Philippines. \$\$.

P. (Phym.) zosteræforme, Wall. Hab. . Tenasserim, Noulmein. $\$$.

P. (Phym.) lycopodioides, Lim. Hab. : Java.

P. (Phym.) normale, Dom. Hab. . Moulmein, Malaya.

P. (Phym.) myriocarpum, Mett. Hab.: Philippines, Borneo; Sarawak, on limestone. Cochin China. \$.

P. (Phym.) lingureforme, Mett. Hab.: Amboyna.

P. (Phym.) rhynchophyllum, Hook. Hab. : Moulmein, 7,000 feet.

P. (Phym.) triquetrum, $B l$. Hab. : Java. \$

P. (Phym.) rupestre, Blume (non R. Br.; P. saxatile, Mett.). Hab. : Java and Philippines. \$\$.

P. (Phym.) platyphyllum, Sw. (P. crassinervium, Bl.). Hab. . Java. \$

P. (Phym.) heterocarpum, Blume. (non Mett.). Hab.: Java, Borneo, Philippines.

P. (Phym.) irioides, Lam. Hab.: Malay Archipelago, Java, Philippines. Hook. Fil. Exot. t. 4. boyna.

P. (Phym.) musæfolium, Bhume. Hab.: Java, Sumatra, Am- 
Polypodiun-continued.

P. (Phym.) Labrusca, Hook. Hab. : Borneo, on limestone rock ; Sarawak. \$.

P. (Phym.) Spectrum, Kaulf. Hab.: Sumatra.

P. (Phym.) pteropus, Bhime. (P. tridactylon, Wall.). Hab.: Malay Islands, Isle of Samar, Java, Mergui, Moulmein.

P. (Dipteris) Dipteris, Blume. (P. IIorsfieldii, R. Br.). Hab. : Malay Islands; Penang, Java, Luzon, Singapore, Borneo.

P. (Dipt.) Lobbianum, Hook. Hab. : Mount Ophir, Malayan Peninsula and Sarawak, Borneo. \$.

P. (Phym.) incurratum, Blume. Hab.: Java, Mount Ophir, Straits of Malacea. \$.

P. (Phym.) insigne, Blume. Hab.: Java, Philippines and Malacca. \$.

P. (Phym.) phymatodes, Linn. Hab. . Malay Islands, Java, Singapore, Borneo, Amboyna, Malacca.

P. (Phym.) nigrescens, Blume. Hab.: Malayan Islands, most abundant. Java, Moluccas, Amboyna, Luzon. Hook. Fil. Ercot. t. 22 .

P. (Phym.) affine, Bhume. Hab.: Malaccas and Philippines; Java, Sumatra, Celebes, and Luzon. \$.

P. (Phym.) dilatatum, Wall. Hab.: Malayan Peninsula, Moulmein; ascent of Mooleit, on rocks, alt. 4,000 feet.

P. (Phym.) laciniatum, Blume. Hab. : Java. \$\$.

P. (Phym.) glaucum, Kze. Hab.: Philippines. \$8.

P. (Phym.) lomarioides, Timze. Hab.: Java, Borneo, Philippines, Luzon. \$.

P. (Phym.) longissimum, Blume. Hab.: Java, Celebes, Luzon, Moulmein.

P. (Drynaria) Heracleum, Kze. Hab.: Java and Philippines. Hook. Gard. Ferns, t. 1. \$

P. (Dry.) Meyenianum, Schott. Hab.: Philippines, Luzon, on Mount Baldu. \$

P. (Dry.) conjugatum, Lam. (P. coronans, Irall.). Hab.: Moulmein.

P. (Dry.) splendens, Hook. Hab. : Philippines and Singapore. \$ $\$$

P. (Dry.) propinquum, Wall. (P. Wildenovii, Bory.). Hab.: Java. Hook. Gard. Ferns, t. 35.

P. (Dry.) quercifolium, Linn. Hab.: Moulmein, Pegu, Penang, Singapore, Malay Isles, Java, Borneo, Luzon. Hook. Gard. Ferns, t. 5 .

P. (Dry.) Linnæi, Bory. Hab.: Malacca, Java, Sumatra, Celebes.

P. (Dry.) rigidulum, Swartz (P. diversifolium, $R$. Br.). Hab.: Malay Peninsula and Islands.

P. (Phymatodes) palmatum, Blume. Hab.: Java, Island of Ternate, Luzon, Singapore, Penang. \$.

P. (Phym.) I,ehmanni, Mett. Hab.: Burnah.

P. (Phym.) albo-squamatum, Bheme. Hab.: Java, Borneo, Sarawak, Luzon. Hook. Gard. Ferns, t. 47. \$.

\section{Monogramme.}

M. dareæcarpa, Hook. Hab.: Labuan; Borneo, on the trunks of trees. $\$$. 
Monogramme-continued.

M. trichoidea, J. Sm. Hab. . Luzon, Philippines. \$.

M. Junghuhuii, Hook. Hab. : Java, Luzon.

\section{Gymnogramme.}

G. (Leptogramme) Totta, Schlecht. (G. Lowei, Hook. \& Gr. Ic. Fil. t. 89). Hab. : Java.

G. (Lept.) opaca, Spreng. (G. obtusata, Bl.). Hab. : Java.

G. (Digrammaria) ambigua, Hook. Hab. : Philippines. $\$$.

G. (Stegnogramme) aspidioides, Hook. (non Kuulf.). Hab.. banks of rivers; Java, alt. 3-4,000 feet. Hook. C'ent. Ferus, t. 50.

G. Javanica, Blume. Hab.: Malayan Isles, Java, Luzon, Sumatra, Moulmein.

G. (Syngramme) Borneensis, Hook. Hab.: on trees, Sarawak; Bormeo, alt. 2,500 feet. $\$$.

G. (Syn.) cartilagidens, Baker. Hab.: Borneo. $\$$.

G. (Syn.) obtusitolia, Houk. Hab. : Java. \$.

G. (Күн.) Lobbiand, Hook. Hab. : Borneo. \$.

G. (Syn.) vittæformis, Hook. Hab. . Philippines, Island of Samoa. \$\$.

G. (Syn.) Wallichii, Hook. Hab. : Singapore, Borneo, Sarawak and Labuan. $\$$.

G. (Syn.) alismæfolia, Hook. Hab.: Singapore and Philippines, Luzon. \$s.

G. (Syr.) quinata, Hook. Hab. . Borneo, Sarawak.

G. (Selliguea) lanceolata, Hook. Hab. : Java.

(i. (Sell.) involuta, Don. Hab.: Moulmeiu; Java, frequent. Penang, Borneo, and probably all the Malay Islands; Luzon.

G. (Sell.) avenia, Bater. Hab.. Malayan Penimsula and Islands. $\$$.

G. (Sell.) membranacea, Hook. Hab. . Malay Isles and Philippines; Java, Luzon, and Samar. $\$$.

G. (S'ell.) regularis, Baker. Ilab. : Borneo. \$.

G. (Sell.) macrophylla, Hook. Hab.: Malay and Philippine Isles, Java, Isle of Bohol; Borneo, forest at Sarawals.

G. (Sell.) spuria, Baker. Hab.: Celebes. \$.

G. (Sell.) F'éei, Hook. Hab. . Penang and Singapore, Jara, Borneo. \$.

G. (Sell.) heterocarpa, Blume. Hab. : high mountains of Java. \&.

G. (Sell.) caudiformis, Hook. Mab. : Mergui, Moulmein, Malayan Isles, Java.

G. (Sell.) elliptica, Baker (G. decurrens, Hook.). Hab. : Moulmein, Luzon.

G. (Sell.) pinnata, Hook. Ilab.: Island of Tobir, Indian Archipelago.

\section{Brainea.}

B. insignis, Hook. Hab. : Burmah.

Meniscium.

M. triphyllum, Sw. Hab.: Mishmee and Malacca; Luzon and Leyte, Java, Singapore.

M. salicifolium, Wall. Hab.: Penang and Singapore. Hook. Cent. Ferns, t. 90.

M. cuspidatum, Blume. IIab. : Burmal, Malaccas and Philippines. 


\section{Antrophyum.}

A. subfalcatum, Brack. (A. Brookei, Hook. Second Cent. Ferns, t. 79). Hab. : Borneo. Isles.

A. coriaceum, Wall. Hab.: Mergui, Malayan and Philippine

A. plantagineum, Kaulf. (A. partulum, Bl.). Hab.: Malayan and Philippine Isles, Penang.

A. Cumingii, Fée. Hab.: Luzon. \$.

A. reticulatum, Kaulf. Hab.: Penang, Mishmee, Malaccas.

A. semicostatum, Blume. Hab.: Malay Islands, Borneo, Philippine Islands.

A. latifolium, Blume. Hab.: Java.

\section{vittaria.}

V. elongata, Swz. (V. amboinensis, Fée.). Hab.: Burmah, Amboina.

V. (Tæniopsis) falcata, Kurze. Hab.: Java, Mount Ophir, Malacca.

V. (Tæniop.) lineata, Sw. Hab.: Borneo, Singapore.

V. (Tæniop.) scolopendrina, Thw. (V. Zeylanica, Fée.). Hab.: Philippine and Malayan Isles.

\section{Taenitis.}

T. obtusa, Houk. Hab. : on sandstone rocks, Sarawak ; Borneo, alt. 2,000 feet. Hook. Cent. Ferns, t. 94. \$\$.

T. blechnoides, Sw. Hab.: Malaccas, Philippines, Penang, Luzon, Borneo, Mergui, and Tavoy; Singapore. \$\$.

\section{Drymoglossum.}

D. piloselluides, Presl. Hab. : Malay Islands, Malacca, Singapore, Tenasserim. Hook. Gard. Ferns, t. 46.

D. rigidum, Hook. Hab.: Borneo, near Sarawak. Hook. Cent. Ferns, t. 96.

\section{Femlonitis.}

H. cordata, Roxb. (H. cordifolia, Hook.). Hab.: Moulmein, Luzon, Philippines. Hook. \& Gr. t. 64 ; Hook. Fil. Exot. t. 35.

\section{Acrostichum.}

A. microdenium, Fée. Hab.: Sumatra.

A. conforme, Sw. Hab. : Java, Luzon.

A. Norrisii, Hook. Hab.: Penang. \$.

A. decurrens, Desv. Hab.: Philippines. \$.

A. viscosum, Sw. Hab. : Java, Luzon.

A. Cumingii, Fée. Hab. : Philippines. \$.

A. squamosum, $S w$. Hab. . Sumatra.

A. (Stenochlæna) sorbifolium, Linn. Hab. Malaccas and Philippine Islands, Singapore, Cochin China. Java.

A. (Steno.) scandens, J. Sm. Hab. : Cochin, Singapore, Luzon,

A. (Steno.) laurifolium, Hook. Hab.: Philippines.

A. (Polvbotrya) articulatum, Hook. Hab.: Philippines. \$.

A. (Polyb.) apiifolium, Hook. Hab. : Philippines. \$8.

A. (Egenolfia) appendiculatum, Willd. Hab. : Moulmein to Malaccas and Philippines.

A. (Stenosemia) auritum, Siv. Hab.: Philippine and Malay Isles, (Polybotryn.) Hook. Fil. Exot. t. 81. 
Acrostichum-continued.

A. (Gymnopteris) Linnæanum, Hook. Hab.: Malay Isles, Jara, Borneo. Ilook, ferond Cent. Fern., t. 26. \$.

A. (Gym.) variabile, Hook. Hab. : Jara, Penang.

A. (Gym.) quercifolium, Retz. Hab.: Cochin China.

A. (Gym.) tacciofolium, Hook. Hab.: Philippines, Luzon, Manilla, Isle of Mindoro. (G. trilobata, Sn.) Hook. Cent. Ferns, t. $7 . \$$.

A. (Gym.) Alagelliferum, Trall. Hab. . Moulmein, Java, Luzon.

A. (Gym.) repandum, Ihlume. Hab.: Java, Luzon, Isle of Bonin.

A. (Gym.) subrepandum, Hook. Hab.: Luzon, Penang. \$.

A. (Gym.) Preslianum, Hook. Hah. . Philippines, $\$$.

A. (Gym.) virens, Wall. Hab.: Moulmein, Mortaban.

A. (Chrysodium) minus, Mett. IIab. : Isle of Samar, Philippines. Hook. Second C'cnt. Ferns, t. 86.

A. (Chrys.) lanceolatum, Hook. Hab.: Moulmein, Philippines.

A. (Chrys.) axillare, Cav. Hab.: Philippine Islands, Luzon, Java, Borneo, Moulmein.

A. (Chrys.) bicuspe, Hook. Hab. : Java, on trees.

A. ('hrys.) Blumeanum, Hook. Hab. : Java, Philippines.

A. (Chrys.) aureum, Linn. Hab. . Malay Islands and Peninsula, Borneo.

A. (Hymenolepis) spicatum, Lim. Hab.: Philippines, Jara, Penang. Hook. Gard. Ferns, t. 3.

A. (Hymen.) platyrhynchos, Hook. Hab. Luzon. Hook. Cent. Ferns, t. 99 . \$.

A. (Photinopteris) rigidum, Itall. Ilab.: Singapore, Java, Mindora, Isle of Bohol, Labuan, Luzon, Isle of Panay.

A. (Photi.) drynarioides, Hook. Hab. : Penang. \$\$.

\section{Platycerium.}

P. grande, J. Sm. Hab.: Philippines, Singapore. Hook. Fit. Exot. t. 86.

P. Wallichii, Hook. Hab.. Malay Peninsula, on trees; banlss of the Irawaddy and of the river Martaban; Moulmein. $\$$.

P. biforme, Blume. Ilał.: Malny Islands, Java, Borneo, Malay Peninsula, Mergui, Singapore, Philippines. \$.

\section{Osmunda.}

O. javanica, Blume. Hab. : Java.

\section{Schizzea.}

S. Malaccana, Baker (S. propinqua, Presl. in part). Hab. : Philippines, Malay Peninsula and Isles. Moulmein; on Mount Gedé, 3,500 feet. Borneo. \$.

S. dichotoma, Sw. Hah. : Philippines, Malaccas.

S. (Actinostachys) digitata, Sw. Hab.: Bonin, Philippines, Malay Isles, Mergui. Frook. Gard. Ferns, t. 49.

\section{Iygodium.}

L. dichotomum, Sw. Hab. . Malay Peninsula and Isles, Philippines.

L. semihastatum, Desv. Hab.: Philippine Isles.

L. scandens, Sw. Hab. : Nalay Isles.

L. pinnatifidum, Sw. Hab. . Philippines, Malaccas. 
Lygodium-continued.

L. polystachyum, Wall. Hab.: Malay Peninsula and Isles; woody mountains of Pulo-Penang, Mergui, Tonglow, Moulmein. Hook. Second Cent. Ferns, t. 76 .

L. japonicum, $S w$. Hab.: Java, Philippines.

\section{Angiopteris.}

A. evecta, Hoffm. Hab.: Malay Islands, Luzon, Amboyna. Hook. Fil. Exot. t. 75.

\section{Marattia.}

M, fraxinea, Smith. Hab.: Philippines, Malaceas,

\section{IKaulf ussia.}

K. revculifolia, Blume. (K. Assamica, Griff.). Hab.: Philippine and Malay Isles.

\section{Ophioglossum.}

O. nudicaule, L. fil. Hab.: Malaccas.

O. reticulatum, Linn. Hab.: Malayan and Philippine Isles.

O. (Ophioderma) intermedium, Hook. Hab.: Borneo. \$. Hook. (ent. Ferns, t. 95.

O. (Ophiod.) pendulum, Linn. Hab.: Malaccas, Philippines.

\section{Helminthostachys.}

H. Zeylanica, Hook. Hab.: Philippines; Malayan and Molucca Islands, frequent. Amboyna, Luzon, Tavoy, Mergui, Java, Borneo. Hook. Second C'ent. Ferns, t. 14 ; and Hook. Gard. Ferns, t. 28.

\section{Botrychium.}

B. daucifolium, Trall. Hab. . Java. 


\section{AUSTRALIA AND POLYNESIA.}

1. TROP. AUSTRALIA, N. GUINEA, CAROLINE AND SOLOMON ISLES, N. HEBRIDES, N. CALEDONIA.

2. TEMPERATE AUSTRALIA, TASMANIA, NEW ZEALAND, ETC. 3. POLYNESIA, FRIENDLY, SOCIETY, SANDWICH, MARQUESAS ISLES, ETC.

$$
\$ \text { denotes the only hitherto known localities. }
$$

\section{TROPICAL AUSTRALIA, NEW GUINEA, CARO- LINE AND SOLOMON ISLES, NEW HEBRIDES, NEW CALEDONIA.}

\section{Platyzoma.}

P. microphyllum, $B r$. Hab.: Tropical Australia; in barren districts about Gulf of Carpentaria, and Arnhem's Land. F. Mueller.

\section{Gleichenia.}

G. moniliformis, Moore. Hab.: N. Caledonia. \$s.

G. circinata, Swuz. (G. speluncæ, Br.). Hab.: N. Caledonia.

G. rupestris, Br. Hab.: NW. Australia, Elizabeth Bay, New Caledonia.

G. dicarpa, Br. (G. alpina, Br.; Hook. \& Gr. Ic. Fil. t. 58). Hab. . Isle of Pines, New Caledonia.

G. flabellata, $B r$. Hab.: Rockingham Bay, Queensland; New Caledonia, New Ireland.

G. dichotoma, Willd. Hab.: Mount Hinchinbrook and Rockingham Bay ; Arnhem's Land, at the cataracts of the river Victoria. -F. Mueller. New Caledonia, New Ireland, Isles of Pines.

\section{Cyathea.}

C. integra, $J$. Sm. IIab. : New Ireland.

C. Lindsayana, Hook, Hab.: NE. Australia. (The first true Cyather found in Australia.) \$.
C. Aneitense, Hook. Hab.: Isle of Aneiteum, N. Hebrides.
C. Vieillardii, Mett. Hab.: Balade, New Caledonia. \$\$.
C. nigricans, Mett. Hab. . Ualau, of the Caroline Islands. \$.
C. leucolepis, Melt. Hab. : Aneiteum and Solomon's Group.

\section{Alsophila.}

A. Samoensis, Brack. Hab. . Louisiade Archipelago. \$. 
Alsophila-continued.

A. decurrens, Hook. Hab.: Pacific Isles, Aneiteum, Samoan Isles, New Caledonia. \$.

A. Novæ Caledoniæ, Mett. Hab.: New Caledonia.

A. australis, $\boldsymbol{B r}$. Hab. Louisiade Archipelago. Bay.

A. Robertsiana, F. Mueller. Hab.: Queensland, Rockingham

A. Rebeccæ, F. Mueller. Hab. : Sea-coast range at Rockingham Bay, F. Mueller.

A. lunulata, Br.? Brack. Hab.: New Caledonia, Aneiteum.

\section{Dicksonia.}

D. antarctica, Labill. Hab. . New Caledonia.

D. Berteroana, Hook. Hab. : New Caledonia.

D. dubia, Gaud. (D. straminea, Labill.). Hab. : New Caledonia, Aneiteum.

D. Haccida, Swz. Hab. : Aneiteum, New Hebrides. \$.

Deparia.

D. Moorei, Hook. Hab. : New Caledonia. Hook. Exot. Ferns, t. $28 . \$$.

\section{Fymenophyllum.}

H. mnioides, Baker. Hab. : New Caledonia, Pic du Mont-Mu. \$.

H. demissum, Swz. Hab. : near Rockingham Bay. F. Mueller.

H. dilatatum, Swo. Hab. . Aneiteum.

Trichomanes.

T. peltatum, Baker. Hab.: New Caledonia.

T. Motleyi, $V . D . B$. Hab.: New Caledonia.

T. parvulum, Poir. Hab. : New Ireland.

T. saxifragoides, Presl. Hab.: New Ireland.

T. pyxidiferum, $L$. Hab. . New Caledonia. Hook. \& Gr, $l_{c}$. Fil. t. 206.

T. rigidum, Suz. Hab.: Sea-view range, Rockingham Bay. $F$. Mueller.

'I. fœniculaceum, Bory. (T. setilobum, F. Mueller, MSS.). Hab. . Rockingham Bay.

\section{Davallia.}

D. pectinata, Smith. Hab. : Island of Jobie, Coral Islands. Hook. \& Gr. Ic. Fil. t. 139.

D. pedata, Smith. Hab.: Queensland, Rockingham Bay.

D. pusilla, Metten. Hab. : Aneiteun, New Caledonia. \$.

D. botrychioides, Brack. Hab. : Aneiteum.

D. Moorei, Hook. Hab.: Canalla, New Caledonia. Hook. Second Cent. Ferms, t. $53 . \$$.

D. solida, Swz. Hab.: New Ireland, New Hebrides, Tanna, New Caledonia, Island of Vanikoro, New Guinea.

D. elegans, Swz. Hab.: Tropical Australia, Dalrymple's Gap, Castle Mount at Cleveland's Bay. F. Mueller.

D. pyxidata, Cav. Hab. : Coral Islands.

D. inæqualis, Kunze. Hab.: Aneiteum.

D. Speluncæ, Baker (D. polypodioides, Hook.). Hab.: Queensland, Broad Sound, Banks Island, New Hebrides. F. Mueller.

D. scoparia, Hook. MSS. Hab.: New Caledonia. \$. 


\section{Iindsaya.}

L. linearis, Swæ. Hab. . New Caledonia.

L. cultrata, Su: Hab.: Queensland.

L. Aabellulata, Dry. Hab.: Port Essington.

L. lanuginosa, Wrall. Hab. : Edgecumbe Bay, in rocks exposed to the spray of the sea; New Guinea.

L. alutacea, Metten. Hab.: New Caledonia. \$.

L. trichomanoides, Dry. Hab.: Rockingham Bay. F. Miteller.

L. elongata, I ab. Hab. . New Hebrides, New Caledonia, Isle of Pines. \$.

L. retusa, Metten. IIab. : N'ew Caledonia, Solomon Isles.

L. media, $R$. Br. Hab.: tropical shores of Australia and adjacent isles. Hook. Cent. Firns, t. 57. \$.

L. nitens, Blume. (L. recurvata, If all.). Hab. : Queensland.

L. cordata, Gaud. Hab. - New Guinea.

L. Fraseri, Hook. Hab. : Queensland, Cleveland Bay.

Adiantum.

A. Iunulatum, Burm. Hab.: Rockingham Bay.

A. diaphanum, Blume. Hab.: New Caledonia, Aneiteum.

A. pulchellum, Blume. Hab.: Aneiteum.

A. Ethiopicum, L. Hab. : Broad Sound. F. Mueller.

A. hispidulum, Swz. Hab.. Rockingham Bay. F. Mueller.

\section{Fy polepis.}

Islands.

H. tenuifolia, Bernh. Hab.: Tauna Isle, New Hebrides, Coral

\section{Cheilanthes.}

Ch. Sieberi, Kunze. Hab.: New Caledonia, Isle of Pines.

\section{Pellaea.}

I' geranirefolia, Fée. Halo.: Bersaler's Range, Point Cooper, New Caledonia, Aneiteum.

\section{Pteris.}

Pt. longifolia, $L$. Hab.: New Hebrides; Aneiteum, Isle of Pines; near the town of Rockhampton on Bersaker's Range. $F$. Mueller.

Pt. crenata, Suz. Hab. ' New Hebrides; Aneiteum, abundant.

Pt. quadriaurita, Retz. Hab. . Rockingham Bay. $F$. Mueller.

Pt. Nova-Caledonie, Hook. Hab.: New Caledonia, on the ground, in low, moist situations. \$.

Pt. aquilina, $L$. Hab. : Rockingham Bay, Isle of Pines.

Pt. rugulosa, Labill. IIab.: New Caledonia.

Pt. Feliciennæ, F. Muell. Hab.: Rockingham Bay, in woody places.

Pt. Vieillardii, Metten. Hab. : New Caledonia. \$3.

Pt. Milneana, Baker. Hab. : Solomon Isles.

Pt. incisa, Thunb. Hab.: Aneiteum.

Pt. tripartita, Swz. Hah.: Rockingham Bay, Isle of Pines.

\section{Ceratopteris.}

C. thalictroides, Brong. Hab. : Roper's River; Gulf of Carpentaria, in still waters. Albert River, Fitzroy River, Burdelrin's River, Pine River. 
Iomaria.

L. Patersoni, Spreng. Hab. . Rockingham Bay, Aneiteum.

L. Vieillardii, Baker. Hab.: New Caledonia. $\$$.

L. ciliata, Moore. IIab. : New Caledonia. $\$$.

L. gibba, Lalill. Hab. : New Caledonia; Isle of Pines, on margins of streams, and in Aneiteum. $\$ 8$.

L. opaca, Baker. Hab.: New Caledonia, Aneiteum. \$.

L. lanceolata, Spreng. Hab.: New Caledowia.

L. obtusata, Labill. Hab. : New Caledonia. \$\$.

L. procera, Spreng. IIab. . volcanic mountain Ianna, N. Hebrides.

L. diversifolia, Baker. Hab. : New Caledonia. \$

L. Lenormandi, Baker. Hab. : New Caledonia. \$.

\section{Blechnum.}

B. cartilagineum, Swz. Hab.: Rockingham Bay.

B. nitidum, Presl. Hab. : Isle of Guadalcomar; Solomon Isles, in woods.

B. serrulatum, Rich. (B. striatum, Br.). Hab. : Port Essington, New Caledonia.

B. orientale, Linn. Hab. : Adelaide River, Arnhem's Land, Sen-view range, Rockinghan Bay, Nount Ilinchinbrook. $F$. Mueller.

Doodia.

D. aspera, R. $B r$. Hab.: Port Essington; from Snowy River to Broad Sound. F. Mueller.

D. media, $R$. Br. (U. connexa, Kze.). Hab. . Coral Isles.

D. caudata, $R$. Br. Hab.: Rockingham Bay.

\section{Asplenium.}

A. Nidus, L. Hab. : Queensland, and through all Eastern Australia, south to Two-fold Bay,-F. Mueller. New Caledonia, New Hebrides.

A. Fejeense, Brack. Hab.: Aneiteum.

A. simplicifrons, $F$. Muell. Hab.: on the moist summit of Mount M'Allister, along the streams flowing into Rockingham Bay. \$.

A. Vieillardii, Mett. Hab. : New Caledonia. $\$$.

A. persicifolium, J. Sm. Hab.: Gondalon Island, Solomon's Group.

A. rhizophorum, Linn. Hab.: Solomon's Group.

A. hirtum, Kaulf. Hab. : Solomon Islands.

A. paleaceum, R. Br. Hab. : Bersaler's Range, NE. Australia, Frankland Isles, $\$$.

A. falcatum, Lam. Hab. . Port Essington, Pig Island, Louisiade, Isle of Pines, Aneiteum and Fortuna Islands.

A. macrophyllum, Swz. Hab.: Pig Island, Louisiade.

A. affine, Sivz. (A. spathulinum, J. Sm.). Hab.: Aneiteum.

A. laserpitiifolium, Lam. Hab.: Fitzroy Island, Rockingham Bay, Solomon's Group, Isle of Pines.

A. bulbiferum, Forst. Hab. : New Caledonia.

A. (Darea) obtusilobum, Hook. Hab.: Aneiteum and Tanna. Hook. Cent. Ferns, t. 100.

A. (Darea) Noræ Calodoniæe, Hook. Hab.: New Caledonia, 
Asplenium-continued.

crevices of rocks in very dry and exposed places. Hook. Cent. Ferns, t. 11. \$.

A. (Darea) scandens, J. Sm. Hab.: New Guinea.

A. (Dipl.) bantamense, Baker (A. fraxinifolium, Wall.). Hab. : Aneiteum. Hook. Second C''nt. Ferns, t. 19.

A. (Dipl.) cyatheæfolium, Bory. Hab. . Ualan, New Guinea.

A. (Iipl.) polypodioides, Mett. Hab. : in the valleys near Rockingham Bay. F. Mueller. teum.

A. (Dipl.) melanocaulon, Baker. Hab.: Dalrymple's Gap, Anei-

A. (Aniso.) decussatum, Swz. Hab.: Solomon's Group, New Ireland, Jobie Island.

\section{Aspidium.}

A. (Polyst.) aristatum, Swz. Hab.: Rockingham Bay. F. Mueller. Nephrodium.

N. (Listrea) obliquatum, Baker. Hab. : New Caledonia. \$.

N. (Last.) subsericeum, Baker. Hab.: New Caledonia. \$.

N. (Last.) decompositum, $R$. Br. Hab.: Rockingham Bay, inhabiting moist woods. F. Mueller.

N. (Last.) Vieillardii, Baker. Hab. : New Caledonia. \$.

N. (Last.) membranifolium, Presl. Hab.: New Caledonia.

N. (Last.) setigerum, Baker (N. tenericaule, Hook.). Hab.: Rockingham Bay, Broad Sound. F. Mueller.

N. unitum, R. Br. Hab. : Rockingham Bay, in marshes among Screw Pines; Providence Hill in Arnhem's Land. F. Mueller.

N. pteroides, J. Sm. Hab.: Queensland.

N. arbuscula, Desv. Hab. : Solomon Isles.

N. truncatum, Presl. Hab. : Solomon Isles.

N. (Sagen.) Meyanthidis, Baker. Hab.: Solomon Isles, New Guinea.

N. (Sagen.) decurrens, Baker (Aspidium pteropus, Kunze). Hab. . Aneiteum.

N. (Sagen.) pachyphyllum, Baker (Aspid. grande, J. Sm.). Hab. : Solomon Isles.

\section{Nephrolepis.}

N. exaltata, Schott. Hab. : Edgecumbe Bay, Rockingham Bay, Queensland, Teviot River, Isle of Pines.

N. acuta, Presl. Hab.: Queeusland, Isle of Pines, Aneiteum, Navigators Island.

N. ramosa, Baker (N. obliterata, Hook.). Hab. . Endeavour River, Rockingham Bay.

N. altescandens, Balier (Polyp., Coll.). Hab.: Queensland.

\section{oleandra.}

O. neriiformis, Cav. Hab. . New Guinea, Aneiteum.

\section{Polypodium.}

P. (Pheg.) rufescens, Blume. Hab.: Queensland, New Caledonia.

P. (Pheg.) punctatum, Thunb. (P. rugulosum, Labill.). Hab. : Queensland, New Caledonia.

P. (Goniopteris) poecilophlebium, Hook. Hab.: Dunk Island, Rockingham Bay. $\$$. 
Polypodium-continued.

P. (Goniopt.) Kennedyi, F. Muell. Hab.: shady slopes of mountains near Rockingham Bay. F. Mueller. \$.

P. (Goniopt.) urophyllum, Wall. Hab.: Queensland, Aneiteum.

P. (Goniopt.) proliferum, Presl. Hab.: on Mount Muelleri, near Edgecumbe Bay ; New Caledonia.

P. (Dictyopteris) ferrugineum, Baker. Hab.: New Guinea. \$.

P. Hookeri, Brack. Hab.: Queensland.

P. (Gram.) australe, Mett. Hab.: Queensland, New Caledonia.

P. (Gram.) Deplanchei, Baker. Hab. : New Caledonia. \$.

P. cucullatum, Nees. Hab.: New Caledonia.

P. crassifrons, Buker. Hab. : New Caledonia. \$.

P. lasiostipes, Mett. Hab. : New Caledonia. \$.

P. blechnoides, Hook. Hab. : Queensland, Rockingham Bay.

P. (Goniophlebium) subauriculatum, Blume. Hab.: New Caledonia, Queensland.

P. (Niphobolus) serpens, Forst. (nom Swz.; P. rupestre, R. Br.). Hab. : from Snowy River, as far as Burnett's Stream; and Mount Elliot, on mossy rocks and trunks of trees. $F$. Mueller.

P. (Niph.) acrostichoides, Forst. Hab. . Rockingham Bay, Queensland; New Hebrides.

P. (Niph.) stigmosum, Swz. (P. costatum, Wall.). Hab.: New Guinea.

P. (Phymatodes) Cunninghami, Hook. Hab. : Isle of Mallicolla, New Hebrides.

P. (Phym.) sinuosum, Wall. Hab.: New Hebrides, Isle of Jobie, Solomon Isles.

P. (Phym.) Brownii, Wickstr. (P. attenuatum, R. Br.). Hab.: New Caledonia, Isle of Pines. \$ 8 .

P. (Phym.) glabrum, Mett. Hab.: Lord Howe's Island, New Caledonia.

P. (Phym.) angustatum, Swz. Hab.: Coral Islands; Brisbane River, E. Australia.

P. (Phym.) linguæforme, Mett. Hab. . Solomon Isles.

P. (Phym.) lanceola, Mett. Hab. : New Caledonia. \$s.

P. (Phym.) irioides, Lam. Hab.: Broad Sound, Rockingham Bay; New Caledonia, Isle of Pines.

P. (Dipteris) Dipteris, Blume. Hab. . Aneiteum.

P. pustulatum, Forst. Hab.: Tropical Australia.

P. (Phym.) phymatodes, Linn. Hab. : New Guinea.

P. (Drynaria) quercifolia, Linn. Hab.: Queensland, Albany Island, Rockingham Bay. Isles.

P. (Dry.) Linnæi, Bory. Hab.: Tropical Australia, Solomon land.

P. rigidulum, Swz. (P. diversifolium, R. Br.). Hab.: Queens-

\section{wothochlaena.}

N. lanuginosa, Desv. Hab. : Upper Victoria River, and also near Lake Torrens.

N. fragilis, Hook. Hab. : Arnhem's Land; on rocks at Fitzmaurice River, N. Australia. \$.

N. hirsuta, Desv. Hab. : Coral Islands. 
Nothochlcena-continued.

N. distans, R. Br. Hab. : New Caledonia, Isle of Pines. Hook. Second c'ent. Ferns, t. 80.

\section{Monogramme.}

I. subfalcata, Hook. Hab.: Island of Malicolla, New Hebrides. \$.

\section{Gymnogramme.}

(1. marginata, Mett. Hab. : New Caledonia. \$.

(*. Muelleri, Hook. Hab.: Cleveland Bay, Fort Cooper, Queensland. $\$$.

G. decipiens, Mett. Hab. : New Caledonia, Aneiteum. \$\$.;

G. (Selliguea) macrophylla, Hook. Hab. : New Guinea.

G. (Sell.) involuta, Don. Hab. : Solomon Isles.

G. (Sell.) caudiformis, Hook. Hab.: New Caledonia, Aneiteum.

G. (Sell.) palmata, Baker. Hab.: San Christoval, Solomon Isles, $\$$. land.

G. (Sell.) elliptica, Balier (G. decurrens, Hook.). Hab. : Qneens-

\section{Antrophyum.}

A. semicostutum, Blume. Hab.: Dalrymple's Gap.

\section{vittaria.}

V. elongata, Su: Hab.: Queensland, New Caledonia, New Hebrides.

V. (Tæniopsis) scolopendrina, Thw. (V. Zeylanica, Fée.). Irab.: New Guinea.

\section{Acrostichum.}

A. conforme, Sir: Hab.: Queensland, Rockingham Bay.

A. (Stenochlena) sorbifolia, Linn. Hab. . New Caledonia.

A. (Steno.) scandens, J. Sm. Hab.: Queensland.

A. (Steno.) laurifolium, Hook. Hab.: Solomon Isles.

A. (Pulybutrya) Wilkesianum, Hook. Hab. . New Caledonia.

A. (Stenosenia) auritum, Suz. Hab. : Silomon Isles.

A. (Gymnopteris) repandum, Blume. Hab.: New Caledonia: Queensland, Sen-view range; Rockingham Bay, on rocks washed by streanis. F. Alutedler.

A. (C'hrysodium) aureum, Lim. Hab. Queensland, Rockingham bay, Port Essington, Fitzmaurice River. F. Wheller.

A. (Chrys.) polyphyllum, Hook. Hab. : Aneiteum, Tanicolla Isle, New H+brides.

A. (IIrmenolepis) spicatum, Linn. Hab.: Brisbane River, Solomon Isles, New Caledonia.

A. ('hotinupteris) drynarioides, Hook. Hab.: Solomon Isles.

\section{Platycerium.}

P. grande, J. Sm. Hab. : NE. Australia.

\section{Todea:}

T. barhara, Moore (T. Africana, Trilld.; T. rivularis, Sieb.). Hab. . Queensland, rockingham Bay.

T. (Leptopteris) Fraseri, Hook. \& Gr. Hab. : New Caledonia.

\section{Schizaea.}

S. fistulosa, Lalbill. IIab. . New Caledonia 
Schizea-continued.

S. (Loph.) dichotoma, Swz. Hab.: Rockinghaun Bay, Providence Itill, Arnhem's Land. Ferd. Wueller.

S. (Actinostachys) pennula, Swz. Hab.: New Caledonia, Isle of Pines.

\section{Iygodium.}

L. trifurcatum, Baker. Hab.: Solomon Isles, Louisiade Archipelago.

L. scandens, Swz. (L. semibipinnatum, R. Br.). Hab.: Queensland, Roper's River, Rockingham Bay. F. Mueller.

L. (Hydroglossum) reticulatum, Schk. Hab.: East Tropical Australia.

\section{Angiopteris.}

A. evecta, Hoffm. Hab.. New Caledonia; Queensland, Rockingham Bay, to Dalrymple's Creek. F. Mueller.

\section{marattia.}

M. fraxinea, Smith. Hab. : Queensland, Rockingham Bay.

M. attenuata, Lab. Hab. : New Caledonia. \$.

\section{Ophioglossum.}

O. vulgatum, Linn. Hab. : Arnhem's Land and Victoria River. F. Mueller.

O. nudicaule, L. fll. Hab. . New Caledonia.

\section{Felminthostachys.}

H. Zeylanica, Hook. Hab. New Caledonia ; Queensland, Rockingham Bay, everywhere common. F. Mueller.

2. TEMPERATE AUSTRALIA, TASMANIA, NEW ZEALAND, AUCKLAND ISLES, KERMADEE ISLES, CHATHAM ISLAND.

\section{Platyzoma.}

P. microphyllum, $R$. $B r$. Hab.: Yandilla, Darling Downs, Isabella's Creek, Leichbardt Range, Glenelg River, Victoria. $F$. Mueller.

\section{Glelchenia.}

G. circinnata, Swz. (G. Speluncæ, Br.; G. 'semivestita, Lab.). Hab, : Sydney, Broken Bay, Hunter's River, Port Jackson, Moreton Bay. From Rifle Range, near Portland, through Australia F'elix. -F. Mueller. Tasmania; New Zealand, abundant throughout the Northern and Middle Islands; Chatham Island. The natives affirm that this Fern is the first to appear after clearing the forest, and hence call it Matua Rarauhe (father of Ferns). J. D. Hooker.

G. rupestris, Br. Hab. : Port Jackson.

G. dicarpa, $B r$. (G. alpina, Br.; smaller and more compact). Hab.: Port Jackson, Moreton Bay, Broken Bay, Victoria, Australian Alps, Tasmania, Bass's Straits, New Zealand, Northern Island; Lake Taupo, and mountainous districts of the interior. In Middle Island both varieties probably common throughout to Foveaux Straits, Chatham Island. J. D. Hooker.

G. Habellata, $B r$. Hab.: Victoria, Glenelg River, and Grampian 
Gileichenia-continued.

Mountains; Sydney, Moreton Bay, Brisbane River, Hunter's River, Buffalo Range, Tasmania, New Zealand; Northern Island, in shady woods; Bay of Islands. J. D. Hooker. \$.

G. tenela, $B r$. Hab. : Tasmania. 8 .

G. Cunninghami, Hevo. Hab.: New Zealand, common throughout the Northern Island; Middle Island, Canterbury, Stewart's Island. J. D. Hooker. $\$ 8$.

G. dichotoma, Welld. Hab.: Hunter's River, York Sound, Brisbane River, Victoria. New Zealand: Northern Island; hot springs, Karapiti, and at Rotamahana. A most abundant tropical Fern all over the world. J. D. Hooker.

\section{cyathea.}

C. medullaris, Swz. Hab.: Cape Otway ranges, Victoria.-F. Mueller. New Zealand, abundant throughout the Northern and Middle Islands, as far south as Akaroa. 'Black Fern' of the settlers, Chatham Island.-J. D. Hooker. Norfolk Island. Hook. Gard. Forns, t. 25 . \$.

C. Cunninghami, Hook. fil. Hab.: New Zealand; Northern Island, east coast and interior; Auckland; Port Nicholson, in dense forests. $-J . D$. Hooker. Common in the woods of the Chatham Islands, where it is called by the natives 'Ponja.' F. Mueller'; Hook. Cent. Ferns, t. 85.

C. dealbata, Sic $\sim$ Hab.: New Zealand, abundant throughout Northern and Middle Islands. 'Silver Tree-Fern' of the settlers. Lord Howe's Island, common in woods of the Chatham Islands. J. D. Hooker.

C. Milnei, Hook. Hab. : Sunday, or Raoul Island, Kermadee Group. \$8.

C. affinis, Swz. Hab. : Cape Otway Roughs, Victoria.

\section{Femitelia.}

H. Smithii, Hook. Hab. . New Zealand; Northern Island, mountainous districts in the east coast and interior; Wellington, usually near streams; Middle Island, apparently common throughout. J. D. Hooker.

\section{Alsophila.}

A. MacArthuri, Hook. (A. Leichardtiana, F. Mueller). Hab. : from Shoalhaven River as far as Moreton Bay and Maryborough; perhaps found in Gipps' Land. TToods near Sydney, Hastings and Marlony Rivers; Mlawara, Mount Lindsay. 'Whip-stick-Fern' of the colonists. 8 .

A. australis, $B r$. Hab. : from Hopkins River as far as Broad Sound, Hastings and Clarence Rivers, Australian Alps, Victoria; Macquarie Harbour, Tasmania.

A. Colensoi, Hook. fil. Hab. : New Zealand; Northern Island, Ruahine Range; Middle Island, probably common, Southern Alps; Otago, alt. 2,000 feet. J. D. Hooker; Fl. New Zerland, t. 73 . \$.

A. excelsa, Br. Hab. : Illaware, Hastings River; Mount Lindsay, N. S. Wales; Norfolk Island. $\$$.

\section{Dicksonia.}

D. antarctica, Labill. (D. fibrosa and D. lanata, Hz. Sp. Fil.). 
Dicksonia-continued.

Hab. . summit of the Blue Mountains, E. Australia; in limestone caves not far from Mount Gambier, at the mouth of Glenelg River, Victoria-rare; often most abundant through the Fern-tree gullies of Australia Felix and New Cambria ; found northerly at Richmond River and MountLindsay.-F. Mueller. Van Diemen's Land, ravines in the mountainous parts of the island, and close to the sea on the southern shores; New Zealand, in rich alluvial soil near rivers; Northern Island, Bay of Islands; in woods in Te Waiite and Mohaka district; Wairarapa Valley, on acclivities, and in cleared woods on the high shore of the east side of Waikare Lake. Middle Island; Canterbury and Nelson, Otago ; Massacre Bay.

D. squarrosa, Sroz. Hab.: Richmond River, N. S. Wales; New Zealand; Dusky Bay in the Middle Island, southern extremity, extending northward, probably, through the whole of the middle and northern islands; Chatham Island. \$.

D. dubia, Gaud. (D. straminea, Labill.). Hab.: Port Jackson, from Brisbane River as far as the western limits of Victoria. It follows the banks of the rivers, mixed up often with Pteris aquilina. -F. Mueller. Van Diemen's Land.

D. davallioides, $R . B r$. Hab.: Port Jackson, Clarence River, Tasmania.

\section{Deparia.}

D. prolifera, Hook. (D. Macræi, Hook. \& Gr.). Hab.: N. S. Wales, Illawara. F. Mueller.

\section{Ioxsoma.}

L. Cunninghami, Br. Hab.: New Zealand; Northern Island, in woods, rare-very local; Bay of Islands, at the falls of the KeriKeri River; Wangarei River, Waitemata, Coromandel, Thames River. J. D. Hooker; Hook. Gard. Ferns, t. 31. \$.

\section{Hymenophyllum.}

H. marginatum, Hook. \& Gr. Hab. : Port Jackson, among mosses, rare. Hook. \& Gr. Ic. Fil. t. 34 . \$

H. rarum, $R . B r$. Hab. : New Zealand; abundant throughout the Northern and Middle Island, clothing the trunks of Tree-Ferns. - J. D. Hooker. Auckland Isles, Van Diemen's Land. (H. semibivalve, Hook. \& Gr. Ic. Fil. t. 33.)

H. polyanthos, Swz. Hab. : New Zealand, abundant throughout the Northern and Niddle Isles. This Fern has often a strong pecnliar odour when dry. J. D. Hooker.

H. Javanicum, Spreng. (H. crispatum, Wall.; H. Hexuosum, All. Cunn.). Hab. : New Zealand; Northern Island, in woods; Bay of Islands, Middle Island, Canterbury, Otago. Hook. Cent. Ferns, t. 62 ; Hook. \& Gr. Ic. Fil. t. 77.

H. demissum, Swz. IHab. : New Zealand, abundant throughout the Northern and Middle Islands; Dusky Bay, Bay of Islands; Lord Auckland's Group, Chatham Island ; Kermadee Isles.-J. D. Hooker. On mossy stems of Fern-trees of Chatham Island. In Australia, restricted to the south-eastern Fern-tree gullies; frequent in Gipps Land, in moist shady valleys, and thence eastward as far as Hopkins River. In New Cambria, at Wingicarreebee. In the Blue Mountains. F. Mueller.

H. flabellatum, Labill. Hab. : trunks of trees, Tasmania ; and 
Hymenophyllum-continued.

holes of rocks and roots of trees, Nerw Zealand; abundant throughout the Northern and Middle Islands; Lord Auckland Islands, Chatham Island. \$.

H. scabrum, A. Rich. Hab.: New Zealand, Northern Island; Auckland, Bay of Islands; East coast, Middle Island, Nelson and Canterbury, Otago. J. D. Hooker. \$s.

H. dilatatum, s'w. Hab. : New Zealand, abundant throughout the Northern and Niddle Island; Lord Auckland's Group. J. D. Hooker; Hook. \& Gr. Ie. Fil. t. 60.

H. pulcherrimum, Colenso. Hab.: New Zealand; Northern Island, mountains of the east coast and interior; Middle Island, abundant, often pendulous from trees. Hook. ( fil.) Fl. N. Z. t. 74. $\$$.

H. ciliatum, Swz. Hab. : New Zealand; Middle Island, Nelson. J. D. II whier.

II. Franklinianum, Colenso. Hab.: New Zealand, Northern Island; Waikare Lake, Wellington. Mildle Island; abundant in subalpine forests, as far south as Dusky Bay. ( $H$. æruginosum, Carm.) Auckland. J. D. Hooker.

H. Malingii, Metten, WJS's: Hab.: New Zealand, Northern Island; Mount Egmont. Middle Island, mountains between Blind Bay and Massacre or Golden Biy ; Otago; Mount Car Dunedin, alt. 2,000 feet; Francis 'Joseph Glacier, alt. 7,000 feet.J. D. Hooker. (Trichonanes Malingii, Hook. Gard. Ferns, t. 64.) \$.

II. Tunbridgense, Smith.; H. minimum, Rich. (H. antarcticum, Pr.; H. Zeelandicum, V. D. B.). Hab. : N. S. Wales, Australia Felix, Tasnania, Lord Auckland Islands, New Zealand. Throughout the Northern and Mirdle Islands, abundant on roclss and trees; Lord Auckland's Group ( Island, Ruahine Range; Middle Island; common, Southern Alps, Otago (H. minimum, Rivhl.); Northern Island, on roots and stumps of trees; Middle Island, Otago. J. D. Hooker.

H. multitidum, sicz. IIilb.: New Zealand, in woods, on the ground; throughout the islands abundant. Lord Aucliland's Group and Campbell's Island. J. D. Hooker; Hook. \& Gr. Ic. Fil. t. 107.

II. bivalve, Sw: Hab.: New Zealand, Northern Island, east coast and hilly regions of the interior; Middle Island, abundant. J. D. Hooker. $\$$.

\section{Trichomanes.}

T. reniforme, Forst. Hab. : New Zealand, abundant throughout the Northern and Middle Islands. It has been discovered in dustralia. J. D. Hooker; Hook. \& Gr. Ic. Fil. t. 31 ; Fil. Exot. t. 2 . \$\$.

T. calvescens, $T \cdot D . B$. Hab.: N. S. Wales. \$\$.

T. Lyallii, Hook. MSS. Hab.: trees, south-west const of the Middle Island, New Zealind; Thomson's Sound, on trees; Otago, common in the sounds of the west coast on orerhanging rocks; also at Hunua-Huia, twenty-five mles from Auckland. J. D. Hooker. $\$$ s.

T. humile, Forster. Hab.: New Zealand, abundant throughout the Northern and Middle Islands, on trunks of trees; Waimate, Bay of Islands. Hook, \& Gr. Ic. Fil. t. 35. 
Trichomanes-continued.

T. venosum, $R$. Br. Hab. : Victoria; Cape Otway, as far as the eastern limits of Gipps' Land.-Ferd. Mueller. Port Jackson, Tasmania; New Zealand, abundant throughout the Northern and Middle Islands, clothing trunks of Tree-Ferns; Chatham Island. J. D. Hooker; Hook. \& Gr. Ic. Fil. t. $78 . \$$.

T. caudatum, Brack. (T. angustatum, Carm.). Hab.: N. S. Wales; Illawara and Balina, on Richmond River. In the Blue Mountains at Currajong. $F$. Mueller.

T. Colensoi, Hook. fil. Hab.: New Zealand, interior of the Northern Island; dense forests near Waikare; Middle Island, Nelson, Lake Wanaka, George's Sound. J. D. IIockicr; Hook. Cent. Ferns, t. 79.

T. rigidum, Sws, Irab.: New Zealand (T. strictum, Hook. \& Gr. Ic. Fil. t. 122). Northern Island, Hokianga; Middle Island, Dusky Bay, Massacre Bay (var. T. elongatum, A. Cumu.). Abundant in deep shaded woods thronghout the Northern and Middle Islands. J. D. Hooker.

T. meifolium, Bory. Hab. : Norfolk Island.

\section{Davallia.}

D. Novæ Zelandiæ, Colenso. Hab.: New Zealand, Northern Island; Bay of Islands, east const and interior; Wellington, Port Nicholson. Middle Island; Otago, common. J. D. Hooker ;. Hook. Gard. Ferns, t. 51 . $\$$.

D. pyxidata, Cav. Hab. New South Wales, Grampians, Port Jackson, Sydney, Blue Mountains, Moreton Bay, Burnett's River, Rockhampton.-F. Mueller. Norfolk Island. \$. Island.

D. Speluncæ, Baker (D. polypodioides, Hook.). Hab. : Norfolk

\section{Cystopteris.}

C. fragilis, Bernh. (C. Tasmanica, Hook.). Hab.: in the alpine regions of Van Diemen's Land, New Zealand, Northern Island, Mount Egmont ranges. Niddle Island, Wairau and Kailsora Mountains, alt. 3,600 feet; Southern Alps, alt. 3,000 feet; Otago, lake districts. J. D. Hooker; Hook. Cent. Ferns, t. 59.

\section{Iindsaya.}

L. linearis, Swz. Hab.: throughout Australia Felix, and Eastern Australia as far north as Moreton Bay. $-F$. Mrueller. Swan River, W. Australia; Van Diemen's Land. New Zealand, in rocky and stony places in the Northern Island; common at Auckland.

L. trichomanoides, Dry. (L. Lessonii, Bory.). IIab.: N. S. Wales, Illawarra, Tan Diemen's Land; New Zealand, abundant throughout the Norihern and Middle Islands, in woods. J.D. Hooker. \$.

L. microphylla, Svo. Hab. : Temperate Australia, Blue Mountains; Port Jackson, abundant; Glass-house Mountains, Cleveland. -F. Mueller. Brisbane River, New Zealand. Hook.\& Gr. Ic. Fil. t. 194. \$

L. ensifolia, Swz. Hab.: Stradbroke Island, Moreton Bay.

L. Fraseri, Hook. Hab. : Stradbroke Island, Moreton Bay.

Adiantum.

A. diaphanum, Blume. (A. setulosum, J. Sm.). Hab.: N. S. 
Adiantum-continued.

Wales, New Zealand, Norfolk Island.

A. affine, Willd. (A. Cunninghami, Hook.). Hab.: Archer's Creek, Lismore, Richmond River.-F. Mueller. New Zealand, Northern Island; in damp woods, Bay of Islands: Middle Island, Norfolk Island.-J. D. Hooker. Chatham Island; rare, on rocky damp places. $\$$.

A. formosum, R. Br. Hab.: about Port Jackson, in Gipps' Land; New Zealand, Northern Island, banks of the Manganaitaka River; Kaipara and Wangarei. J. D. Hooker.

A. fuivum, Raoul. Hab. : New Zealand, Northern Island ; Bank's Peninsula, off the Middle Island, rather common in dry woods; Norfolk Island, Chatham Island.

A. Ethiopicum, L. Hab.: Temperate Australia; New Zealand, abuudant throughout the Northern and Middle Islands. $J . D$. Hooker.

A. hispidulum, Swz. Hab.: Port Jackson to the tropics; New Zealand; Northern Island, common from the Bay of Islands to Cook's Straits; Norfolk Island, Kermadee Island. J. D. Hooker.

\section{Eypolepis.}

H. tenuifolia, Bernh. Hab.. New Zealand, abundant; Northern Island, Bay of Islands; between Cape Brett and Wangarei Bay. Middle Island, Norfolk Island, Kermadee Islands.

H. distans, Hook. Hab. : New Zealand; Hokianga, on the west coast of the Northern Island, and in the valley of the Hutt and Tararun; Manakau Heads. Middle Island, Canterbury, Otago. J. D. Hooker. \$8.

H. millefolium, Hook. Hab. : New Zealand; woods in a shaded moist dell, near the summit of Ruahine mountain range. Middle Island, Nelson, Lake Rotuiti, Southern Alps, Otago. J. D. Hooker. \$.

\section{Cheilanthes.}

Ch. Sieberi, Kunze. Hab.: Swan River, rocky places, York district; Sydney, Endeavour River; New Zealand, Middle Island, Akaroa.

Ch. tenuifolia, Sw: Hab. : Australia, Port Jackson, Swan River; New Zealand, in stony, rocky places abundant; Bank's Peninsula, Sunk Island; Van Diemen's Land.

\section{Pellaea.}

P. rotundifolia, Hook. Hab. : New Zealand, abundant throughout the Northern and Middle Islands; Bank's Peninsula; Norfolk Island. 8 .

P. falcata, Fée. Hab.: New South Wales; throughout Australia Felix, among rocks in open places. In King's Island. New Zealand, Northern Island; Auckland, Van Diemen's Land, Kermadee Islands.

P. paradoxa, Hook. Hab. : Queensland and N. S. Wales ; Brisbane River, in dry shady woods. \$.

\section{Pteris.}

Pt. longifolia, $L$. Hab. . Rivers Mitchell and Buchan, S. Australia; Glenrock and Bungoma, near Shoalharen : Brisbane River.

Pt. umbrosa, $R$. Br. Hab. : Port Jaclison, Port Stephens; in 
Pteris-continued.

the Blue Mountains, Hastings River, McLeay River, Clarence and Brisbane Rivers. \$.

Pt. tremula, $R$. Br. Hab.: Port Jackson, Illawara, Port Stephens, Victoria ; Lord Howe's Island ; Van Diemen's Land. New Zealand, abundant throughout the Northern and Middle Islands; Auckland, Bay of Islands, Hutt Valley, Wangaroa. \$\$.

Pt. aquilina, L. (Pt. esculenta, Forst.). Hab.: Sydney, Argyll County, Victoria, Flinders Bay, Norfolk Island; New Zealand, most abundant, covering the hill-sides over extensive areas. The var. esculenta, distinguished chiefly by the decurrent pinnules, is common in the south temperate zone. Rhizomes, formerly roasted and eaten by the natives. Tasmania, Raoul, Kermadee Group, Campbell's Island, Chatham Island. J. D. Hooker.

Pt. Scaberula, Richard. Hab. : Now Zealand, Northern and Middle Islands, as far south as Akaroa, frequent; generally in woods; common on open places of Chatham Island.

Pt. macilenta, $A$. Cunning. Hab. : New Zealand, abundant in shady wouds in the Northern Island; Middle Island, Canterbury, Akaroa, \$.

Pt. comans, Forst. (Pt. Endlicheriana, Agardh.). Hab.: New Zealand ; North Island, abundant in woody places; Waikate and Great Barrier Island; Van Diemen's Land ; Norfolk Island, Sunday or Raoul Island (Kermadees). Hook. Cent. Ferns, t. 73.

Pt. incisa, Thung. (Pt. aurita, Bl.). Hab. . N. S. Wales; Mount Disappointment; at Penola, Tatiana country; scattered in moist woods throughout Australia Felix, as far as Clarence River. $-F$. Mueller. New Zealand; North and Niddle Island, abundant on skirts of woods as far south as Akaroa; Lord Auckland's Group and Campbell's Island; Chatham Island; Tasmania.

\section{Iomaria.}

L. Patersoni, Spreng. (L. elongata, Blume.). Hab.: Victoria, S. Australia, frequent on the shady banks of the Broadribb and Cabbage-tree Rivers, and at Sealer's Cove and Bunnip River; Twofold Bay, Illawarra, Blue Mountains, Mount Lindsay; Port Dalrymple, Tasmania. New Zealand, mountainous parts of the Northern Island; Waikare Lake, Port Nicholson, Mount Egmont. Middle Island, Ship Cove, Otago. J. D. Hooker.

L. discolor, Willd. Hab.: St. Vincent's Gulf, Sealer's Cove, Victoria, as far as Port Jackson; Blue Mountains, and Castlereagh River. It occurs also in King's Island.-F. Mueller. New Zealand, throughout the Northern and Niddle Islands, abundant; Lord Auckland's Group and Campbell's Island, Tasmania, in woods of Chatham Island. J. D. Hooker.

L. attenuata, Willd. Hab. : Norfolk Island, Kermadee Islands.

L. vulcanica, Blume. Hab.: Tasmania, in mountain districts; Meander, Mount Wellington, Mt. La Peyrouse; not yet seen in Continental Australia-Ferd. Mueller. New Zealand, Northern Island, on rocks; Tarawera, east coast; Auckland; Taranaki, \&c. Middle Island, Nelson; Otago, subalpine. J. D. Hooker; Hook. Cent. Ferns, t. 69.

L. lanceolata, Spreng. Hab. : New South Wales, Sealer's Core, 
Lomaria-continued.

and in caverns of Nount Gambier, Victoria ; Hopkins River, thence as far as Cape Wilson, scattered through shady woods; Tasmania. New Zealand, abundant throughout the Northern and Middle Islands; Auckland and Campbell's Islands, in woods close to the sea, abundant. J. D. Hooker.

L. dura, Moore. Hab. : Chatham Island.

L. alpina, Spreng. Hab.: abundant in the Alps of S. Australia, in wet bogs by streams ; on Cabbaros, a mountain 6,000 feet elevation, Van Diemen's Land; New Zealand; common in subalpine districts thronghout the Northern and Middle Islands. J. D. Hooker, Fil. Erot. t. 32.

L. Banksii, Hook, fil. Hab.: New Zealand, common in dark woods, throughout the Northern and Middle Islands; Bay of Islands, Auckland. \$\$

L. pumila, Raoul. Hab.: New Zealand, Akaroa, Middle Island. \$.

L. procera, Spreng. (L. Capensis, Willd.). Hab. : S. Australia, from St. Vincent's Gulf, as fir as Mount Lindsay, ascending the sunımit of the Alps; Port Jackson ; Blue Mountains, on shady wet rocks; Macquarrie Harbour and Argyil County, near Melbourne; New Zealand, as far south as Banks Peninsula; Tesmania, extremely abundant; Lord Auckland's and Campbell's Islands ; Raoul or Sunday Island, one of the Kermadee Group; Chatham Island, common in forest ground.-F. Mueller. Gard. Ferns, t. 53. (L. Gilliesii, Hook. \& Gr. Ic. Fil. t. 207.)

L. filiformis, A. Cunn. Hab.: New Zealand, abundant throughout the Northern and Middle Islands, as far south as Bank's Peninsula. (Stenochlæna heteromorpha, J. Smith.)

L. nigra, Colenso. Hab.: New Zealand, Northern Island, in dark moist woods by water courses, east coast and the interior; Middle Island, Milford Haven, and Bligh's Sound. \$. Hook. $C_{\text {int. Ferns, t. } 60 .}$

L. fluriatilis, Spreng. Hab.: Australia Felix, in shady valleys in subalpine regions; not common in Gipp's Land; Delatiti, and Upper Mitta-Mitta.-F. Mueller. New Zealand, Nortbern Island, abundant in the mountainous parts, and throughout the Middle Island; Bank's Peninsula, strongly scented.-J. D. Hooker. Tasmania, Acheron River, \&c. \$.

L. membranacer, Colenso. Hab.: New Zealand; Northern Island, Bay of Islands, common; Waiheki Island, near Auckland; Middle Island, Otago. J. D. Hooker.

L. Fraseri, A. Cunn. Hab.: New Zealand, forests in the Northern Island, and northern parts of Middle Island, at Wangaroa and Hokianga, Massacre Bay, Cook's Straits. \$\$.

L. euphlebia, Kunze. Hab.: by Dallachy, at the sourre of the Mackay River, Australia. Hook. Second Cent. Ferns, t. 89.

\section{Blechnum.}

B. cartilagineum, Swz. Hab.: Port Jackson, Sealer's Cove, Victoria; King George's Sound. \$.

B. lævigatum, Cav. Hab.: N. S. Wales, Port Jackson, River Grose, and in the valleys of the Blue Mountains. F. Mueller. \$. 
Blechrum-continued.

B. serrulatum, Rich. (B. striatum, Br.). IIab. : New Holland, Port Jackson, near McAdam's Range, and between Providence Hill and Point Pierce, Victoria.

\section{Doodia.}

D. aspera, R. Br. Hab.: Port Jackson, Brisbane River, and head of the Burnett River. $\$$.

D. blechnoides, $A$. Cunn. Hab.: N. S. Wales, ravines on the banks of the Nepean, and on the Warra-burrha River. $\$$.

D. media, 'R. Br. Hab. : Endeavour River, shady places; Port Jackson, Snowy River, Australian Alps; New Zealand, Northern Island, on clay hills, \&c., common, fragrant; Norfolk Island.J. D. Hooker. ( $\beta \mathrm{D}$. connexa, Kunze.) Kermadee Islands.

D. caudata, $R . B r$. Hab. : Argyll County, Victoria; Cape Otway, Doodenong ranges, Brisbane River. New Zealand, Northern Island, and Houraki Gulf; Van Diemen's Land.

\section{Asplenium.}

A. Nidus, L. (A. Australasicum, Hook.). Hab.: Moreton Bay, Lord Howe's Isle, Norfolk Island.

A. attenuatum, R. Br. Hab.: Port Jackson, dry and shady woods; Brisbane River, Queensland. \$. Hook. Cent. Ferns, t. 14.

A. flabellifolium, Cav, Hab.: abundant in N. S. Wales, S. A ustralia, as far west as Swan River; Tasmania, plentiful, and in New Zealand; abundant throughout the Northern and Middle Islands, on rocks and in stony places; Bank's Peninsula. J. D. Hooker. \$.

A. Trichomanes, L. Hab. : N. S. Wales, Bathurst, Paramatta; Victoria, Mount Aberdeen. New Zealand, Middle Island; Kaikoras Mountains, Acheron Valley. Canterbury, Southern Alps; Otago, Take district; also in Tasmania. J. D. Hooker.

A. obtusatum, Forst. (A. lucidum, Forst.). Hab. . moist rocky places in N. S. Wales, on Clarence River; Norfolk Island; New Zealand, abundant, Bank's Peninsula; Lord Auckland's Group, generally near the sea; Campbell's Islands, Kermadee Islands.J. D. Hooker. Chatham Island, on rocky places almost everywhere. $F$. Mueller.

A. falcatum, Lam. Hab.: Sydney, Brisbane River; Norfolk Island; New Zealand, most abundant from the north as far south as Alraroa and Port Nicholson; Chatham Island, in woods, on trees and in rocky crevices. $F$. Mueller.

A. caudatum, Forst. Hab. . Brisbane River, Lord Howe's Island; Sunday Island, Kermadee Group.

A. Hookerianum, Colenso (A. Colensoi, Hook. fil.). Hab. : New Zealand, Northern Island, mountains of the east coast and interior; Middle Island, Nelson and Canterbury Alps, Canterbury, Akaroa, Otago, Bank's Peninsula, Mount Maria, Whangarei. J. D. Hooker; Hook. Cent. Ferns, t. 83 and $84 . \$$

A. furcatum, Thunb. Hab. . N. S. Wales, Stirling Range, and south coast of Australia ; Swan River.

A. bulbiferum, Forst. Hab. : Victoria, in caves of Mount Gambier, Glenelg Rirer; Tasmania; New Zealand, abundant in 
Asplenium-continued.

damp woods throughout the Northern and Middle Islands, on the ground, and on rocks and trees; Chatham Island, common in woods on the border of water-courses. F. Mueller. \$.

A. (Darea) Richardi, Hook. fil. Hab.. New River, Middle Island of New Zealand; Port Nicholson. Hook. Cent. Ferns, t. $77 . \$$.

A. (Darea) flaccidum, Forst. IIab.: Blue Mountains, SE. Australia; New Zealand, abundant throughout the Northern and Middle Islands.-J. D. Hooker. Tasmania, Mount Wellington; Lord Auckland's Group, Kermadee Isles; common on trees of Chatham Islands. F. Mueller.

A. (Darea) dimorphum, Kunze. Hab.: Norfolk Island, and nowhere else as yet known. Hook. Second Cent. Ferns, t. 36. \$.

A. (Athyr.) umbrosum, J.Sm. (A. australe, Brack.). Hab. : Port Jackson, Brisbane River, Moreton Bay; Norfolk Island ; Tasmania; New Zealand, Northern Island; in damp woods, frequent, Middle Island, Nelson.

A. (Diplazium) sylvaticum, Presl. Hab.: Richmond River, Dalrymple Gap. F. Mueller.

A. (Dipl.) melanochlamys, Hook. Hab. . Lord Howe's Island (east of N. S. Wales). $\$$.

\section{Aspidium.}

A. (Polystichum) aculeatum, Swz. (A. vestitum, Hook.). Hab.: Sydney, Hunter's River, Clarence River; New Zealand, abundant in the mountainous parts of the Northern and Middle Islands; Bank's Peninsula; Lord Auckland's and Campbell's Islands, from the level of the sea to 1,200-1,400 feet; Macquarie Island. In Tasmania it is most common in subalpine situations, as on Mount Wellington, from 3,000 feet elevation, to the summit. Chatham Island, common in woods, and on the borders of water-courses. F. Afueller.

A. (Polyst.) Richardi, Hook. Hab. : New Zealand, abundant in woods, throughout the Torthern and Middle Islands; sides of cliffs, Tangururu Bay; rocky shores of an island in the Wyran River.

A. (Polyst.) oculatum, Hook. Hab.: New Zealand; Northern Island, Wairarapa Valley, Middle Island, Akaroa, Chathan Island. \$8.

A. (Polyst.) cystostegia, Hook. Hab. : New Zealand, on elevated mountains; Northern Island; Tongariro, Mount Egmont, Middle Island; Discovery Peaks, 5,800 feet; rocks in Waira Gorge, alt. 4,400 feet, in extremely exposed cold situations; between Lake Tennyson and the west coast, Canterbury on the southern Alps, alt. 5-6,000 feet; Otago, Mount Ida, Lake district, alpine. J. D. Hooker. \$.

A. (Polyst.) Capense, Willd. (A. coriaceum, Swoz.). Hab.: New Zealand, Northern and Middle Islands, common in woods; Tasmania.-J. D. Hooker. On tree-ferns of Chatham Island, not common. In Australia, ranging from the vicinity of Cape Otway, enstward to N. S. Wales. $I^{r}$. Mueller.

A. (Polyst.) aristatum, Swz. Hab. : Sydney, Hastings River, Illawarra ; Norfolk Island; Kermadee Isles. 


\section{rephrodium.}

N. (Lastrea) Thelypteris, Desv. Hab. : New Zealand, Northern Island; Bay of Islands, and east coast.

N. (Last.) decompositum, $R . B r$. Hab. : Australia; Victoria, at the village of Penola, in fissures of limestone rocks near Blanchetown, from Cape Otway, as far north as Moreton Bay; New Zealand, Northern and Middle Islands, abundant. Cook's Straits, Tasmania ; Norfolk Island; Chatham Island, common in woods.

N. (Last.) velutinum, Hook. fll. Hab. : New Zealand, abundant throughout the Northern and Middle Islands; Houraki Gulf, and Bank's Peninsula. J. D. Hooker. \$.

N. (Last.) membranifolium, Presl. Hab. : SW. Australia.

N. (Last.) setigerum, Baker (N. tenericaule, Hook.). Hab. : Clarence River, Sydney. $F$. Mueller.

N. (Last.) hispidum, Hook. Hab. : Australia Felix, Cape Otway Ranges, very rare. New Zealand, abundant in Northern and Middle Islands.

N. unitum, R. Br. (non Sieb.; N. propinquum, Br.). Hab.: Melbourne, Moreton Bay, Clarence River, Brisbane; New Zealand, Northern Island, Rotomahana, in a hot water swamp. $J, D$. Hooker.

N. extensum, Hook. Hab. : Duck Creek, on Richmond River. F. Mueller.

N. molle, Desv. Hab. : N. S. Wales; scattered through woody and watered regions of all Eastern Australia, but not found in Australia Felix, except on limestone rocks on the banks of the Murray River, near Blanchetown.-F. Mueller. New Zealand, Northern Island (?). Rotomahana, Kermadee Islands. J. D. Hooker.

N. truncatum, Presl. Hab.: Australia.

\section{Tephrolepis.}

N. cordifolia, Baker (N. tuberosa, Presl.). Hab. : Brisbane Ricer, Clarence River, MrcLeay River. F. Mueller.

N. ramosa, Baker (N. obliterata, Hook). Hab.: Illawarra, Berrima, McLeny River, Richmond River. F. Mueller.

N. altescandens, Baker (Polyp., Hook. Sp.). Hab.: N. S. Wales.

\section{Polypodium.}

P. (Pheg.) punctatum, Thunb. (P. rugulosum, Labill.). Hab. . Van Diemen's Land; Now Zealand, abundant in woods through the Northern and Middle Islands; Lord Auckland's Group, and Campbell's Island; common in the woods of the Chatham Islands; Norfolk Island. J. D. Hooker.

P. (Goniopteris) diversifolium, $S w z$. Hab. : on trunks of trees and rocks in Eastern Australia, Moreton Bay, and Burnett River.

P. (Goniopt.) pennigerum, Forst. Hab. : New Zealand, abundant; Northern and Middle Islands, as far south as Akaroa; common in Chatham lsland, on borders of water-courses. J. D. Hooker. \$.

P. (Grammitis) australe, Mett. Hab.: South Australia, Port Jackson, Mount Lindsay; New Zealand, on trees, rocks, \&c. ; throughout the Northern and Middle Islands, abundant to the extreme south, ascending the mountains to 5,000 feet.-J. D. Hooker. Lord Auckland's and Campbell's Islands ; Tasmania, often at elevations of 4,000 feet.

P. Grammitidis, $R, B r$. Hab. : New Zealand, Northern Island 
Polypodium-continued.

to the extreme south, and in Lord Auckland's and Campbell's Isles, Chatham Island; Tasmania, on trunks of trees, \&c. J. D. Hooker. \$.

P. tenellum, Forst. Hab.: New Zealand, Northern Island, climbing lofty trees, abundant; Middle Island, Cape Farewell; N. S. Wales, from Illawarrd to Moreton Bay, Lord Howe's Island, Norfolk Island. \$.

P. (Gonioph.) amœenum, Wall. Hab.: Hastings River, N. S. Wales. F. Mueller.

P. (Niphobolus) confluens, $R . B r$. Hab.: Australia, Port Jackson and Burnett River. \$.

P. (Niph.) serpens, Forst. (P. rupestre, R. Br.). Hab.: Australia, as far north as Moreton Bay. New Zealand, abundant throughout the Northern and Middle Islands, Bank's Peninsula; Kermadee Islands. J. D. Hooker; Hook. \& Gr. Ic. Fil. t. 44.

P. (Phymatodes) Cunninghami, Hook. Hab.: New Zealand, frequent; Northern and Middle Islands, on trunks of trees, as far south as Akaroa.

P. (Phym.) Brownii, Wickstr. (P. attenuatum, R. Br.). Hab.: Port Jackson, McLeay and Clarence Rivers, Mount Lindsay.

P. (Phym.) glabrun, Mett. Hab. : SE. Australia, as far north as Moreton Bay; Norfolk Island.

P. (Phym.) angustatum, Stcz. Hab.: N. S. Wales.

P. (Phym.) pustulatum, Forst. Hab.: Glenelg River, Victoria; Brisbane River ; Hastings River, in dark forests, climbing to the height of 100 feet; Norfolk Island; New Zealand, Northern and Middle Islands, climbing lofty trees. Plant very fragrant when dry. Chatham Island. J. D. Hooker. \$.

P. (Phym.) Billardieri, R. Br. Hab.: Tasmania, abundant on Mount Wellington, and to the summit; New Zealand, abundant throughout the Northern and Middle Islands, Lord Auckland's Islands, Campbell's Island, Chatham Island, Kermadee Isles.-J. $D$. Hooker. Australia, Norfolls Island. \$\$.

P. (Drynaria) rigidulum, Swz. (P. diversifolium, R. Br.). Hab. : Norfolk Island. Hook. Gard. Ferns, t. 5.

\section{Nothochlzena.}

N. distans, $R$. Br. Hab.: Port Jackson, Logan River, Bathurst county, Port Stephens, Western Australia ; New Zealand, Northern Island; common on basaltic rocks, Middle Island; Maiku Valley, Tasmania. J. D. Hooker; Hook. Cent. Ferns, t. 80.

\section{Gymnogramme.}

G. Pozoi, Kunze (G. rutæfolia, Hook. \& Gr. Ic. Fil. t. 90). Hab.: Australia, from the south-west and east coast to the tropics; New Zealand, east coast, Cook's Straits, on cliffs, very rare; Tasmania. J. D. Hooker.

G. leptophylla, Desv. IIab. : N. S. Wales; Skipton, Port Phillip Head; Mount HcIvor, Barossa Range.-F. Mueller. Tasmania; $\mathrm{New}$ Zealand, Northern Island, hills on the east coast, summit of Mount Wellington, Middle Island, in crevices of rocks.

\section{Acrostichum.}

A. (Hymenolepis) spicatum, $L$. Hab. : woody places at Moreton Bay. $\vec{F}$. Mueller. 


\section{Platycerlum.}

P. alcicorne, Desv. Hab. : N. S. Wales, from Illawarra to Burnett River, Hastings River, Lord Howe's Isle.

P. grande, J. Sm. Hab. : N. S. Wales, Clarence River; Mount Elliot, common near Ipswich. F. Mueller.

\section{Todea.}

T. barbara, Moore (T. africana, Willd.; T. rivularis, Sieb.). Hab.: SE. Australia, from Mount Lofty Range, in moist valleys; Tasmania; New Zealand, Northern Island, Mount Carmel, Hokianga. J. D. Hooker.

T. (Leptopteris) Fraseri, Hook. \& Gr. t. 101. Hab.: Blue Mountains at Fitzroy Waterfall, Australia; rare.

T. (Lept.) hymenophylloides, Rich.\& Less. Hab. : Now Zealand, abundant throughout the Northern and Middle Islands. J. $D$. Hooker; Hook. Gard. Ferns, t. $54 . \$ 8$.

T. superba, Col. Hab.: New Zealand, in forests of the mountainous parts of the Northern Island, more common in the Middle Island; Lord Auckland's Group. J. D. Hooker ; Hook. Cent. Ferns, t. 10 . $\$$.

\section{Schizaea.}

S. australis, Gaud. Hab.: Auckland Islands.

S. fistulosa, Labill. Hab. : Australia, between Cape Wilson and Western Port Bay; Tasmania, New Zealand; Northern Island, Bay of Islands; Middle Island; Canterbury, Otago; Lord Auckland's Group. J. D. Hooker.

S. bifida, Swz, Hab.: Temperate Australia, New Zealand; Northern Island; Bay of Islands, common in wet clayey places; Middle Island, probably common, but overlooked; Otago. J. D. Hooker.

S. (Lophidium) rupestris, R. Br. Hab.: Sydney, Port Jackson, and the Blue Mountains. Hook. \& Gr. Ic. Fil.t. 47 ; Hook. Gard. Ferns, t. 42 . \$.

S. (Loph.) dichotoma, Swz. Hab. . Australia, Port Phillip, Gipp's Land, 'Twofold Bay, Blue Mountains, Port Jackson, Moreton Bay.-F. Mueller. New Zealand; Northern Island, grows at the base of Kaudi trees. J. D. Hooker.

\section{Iygodium.}

L. articulatum, Rich. Hab.: New Zealand, rare, and confined to the northern part of the Middle Island. J. D. Hooker. $\$$. Bay.

L. scandens, Swz. (L. micropbyllum, R. Br.). Hab.: Moreton

\section{Marattia.}

M. fraxinea, Smith. (M. salicina, Sm.). Hab.: New Zealand; forests in the northern and eastern parts of the Northern Island; Taranaki; Norfolk Island. J. D. Hooker.

\section{Ophioglossum.}

O. vulgatum, Linn. Hab.: Australia, from St. Vincent's Gulf and the Kiver Murray, through Australia Felix and Eastern Australia up to the Tropic of Capricorn.-F. Mueller. New Zealand; common in grassy places throughout the Northern and Middle Islands. $J . D$. Hookier.

O. (Ophioderma) pendulum, Linn. Hab.: N. S. Wales, at the source of the Brisbane River, growing on Platycerium. F. Maeller. 


\section{Botrychium.}

B. Luuaria, Swz. JIab.: Australian Alps, at the source of the river Ovens.-F. Mueller. Tasmania.

B. ternatum, $S w z$. (B. cicutarium, Syz.). Hab. : Australia, Tasmania, New Zealand; Northern and Middle Islands.-J. D. Hoolier. On open shady places of Chatham Island. This fern is rery rare in South Australia, and in the lowlands of Victoria and N. S. Wales, extending from Mount Lofty Range to the Tropic of Capricorn. F. Mueller.

\section{POLYNESIA, FRIENDLY, SOCIETY, SANDWICH OR HAWAII,* MARQUESA ISLES, ETC.}

\section{Gleichenia.}

G. longissima, Bl. (Gl. glauca, Hook.;-Mertensia glabra, Brack.). Hab. : Sandwich Isles, Owhyhee.

G. Owhyhensis, Hook. Hab.: Byron's Bay, Owhyhee. \$.

G. Hageilaris, Spr. Hab. : Fiji.

G. dichotoma, Willd. (Mert. emarginata, Brack.). Hab. : Sandwich Isles, Tahiti, Oahu, Owhyhee, Society Isles, Marquesas Isles, Wallis Isle, Feejee Isles.

\section{Cyathea.}

C. medullaris, Swz. Hab.: Pacific Isles, Pitcairn's Island, Otaheite ; Bonin.

C. leucolepis, Mett. Hab. : Samoan or Navigator's Isles, Fiji.

C. affinis, Swoz. Hab.: Pacific Isles; Fiji, Pitcainn's Island.

\section{Memitella.}

H. denticulata, Hook. Hab. : Elizabeth Island, S. Pacific.

\section{Alsophila.}

A. Samoensis, Brack. Hab. : Samoan (or Navigator's) Isles.

A. decurrens, Hook. Hab. . Pacific and Samoan Isles.

A. truncata, Brack. Hab. : Fiji and Samoan Isles. \$\$.

A. Tahitensis, Brack, Hab.: Tahiti. \$\$.

A. lunulata, Br. (?) Brack. Hab.: Pacific Isles, Samoan and Fiji Isles; Bonin Island.

\section{Dicksonia.}

D. (Cibotium) glauca, Smith. Hab.: Oahu, Sandwich Isles; discovered by Menzies in 1807. $\$$.

* 'The Hawaian (or Sandwich) Islands lie just within the northern tropic, between $18^{\circ} 55^{\prime}$ and $220^{\circ} 20^{\prime} \mathrm{N}$. and $154^{\circ} 50^{\prime}$, and $160^{\circ} 40^{\prime} \mathrm{W}$. Their climate is not extreme, being much moderated by the NE. trade winds, which blow pretty $\mathrm{s}^{2}$ eadily for three-fourths of the year. The group comprises thirteen islands. Hawaii (alias Owhyhee), the largest, being about 100 miles long by 80 or 90 miles wide. It also presents the highest mountains of the group, as well as of the Pacific; Mauna Kea being 13,980 feet, and Mauna Loa 13,760 feet: Hualalai somewhat exceeds 8,000 feet. The next island to the west, Maui, is the next in size also. The eastern end is composed of the mountain Haleakala, somewhat exceeding 10,000 feet in height. West Maui is about 6,500 feet high : its mountain is known as Ecka. In Oahu, the mountains are two somewhat parallel ridges, lying nearly east and west: the southern ridge is known as the Kaala Mountains.'-From Horace Mann's Enumeration of Hawaian Plants; a paper drawn up for the Am. Ac. of Arts and Sciences, after a visit to IIawaii in 1864-65. 
Dicksonia-continued.

D. (Oibotium) Menziesii. Hab. : Oahu and Honolulu, Sandwich Islands. \$.

D. (Cibotium) Chamissoi. Hab.: Oahu, Sandwich Isles. \$.

D. Berteroana, Hook. Hab. : Samoa and Fiji. islands.

D. dubia, Gaud. Hab.: Fiji Group, and other Polynesian

Deparia.

D. prolifera, Hook. Hab. : Sandwich Islands, Oahi, Owhyhee. Hook. \& Gr. Ic. Fil. t. 154. \$.

\section{Fymenophyllum.}

H. demissum, Swz. Hab.: Fiji and adjacent Polynesian Islands.

H. recurvum, Gaud. Hab. : Saudwich Islands. \$.

H. dilatatum, Swz. Hab.: Tahiti, Samoa, Fiji, and other Polynesian islands. Hook. \& Gr. Ic. Fil. t. 60.

H. obtusum, Hook. et Arn. Hab. : Oahu, Sandwich Islands. \$s.

H. lanceolatum, Hook. et Aim. Hab.: Oahu, Sandwich Islands. \$.

\section{Trichomanes.}

T. peltatum, Baker. Hab. : Samoan Isles.

T. Vitiense, Baker. Hab. : Fiji Islands. \$.

T. muscoides, Swz. Hab.: Polynesia.

T. parvulum, Poiret. Hab.: Polynesia, Sandwich Isles.

T. saxifragoides, Presl. Hab.: Fiji Isles.

T. Powellii, Baker. Hab. : Samoan Isles. $\$$.

T. digitatum, Swz. Hab. : Polynesian Isles.

T. humile, Forster. Hab. : Society Isles, Otaheite, Pacific Isles. Hook. \& Gr. Ic. Fil. t. 35 .

T. Filicula, Bory. Hab. : Otaheite.

T. radicans, Swz. (T. speciosum, Willd.). Hab. : Polynesian Islands.

T. tenere, Brack. Hab.: Tahiti. \$

T. caudatum, Brack. Hab. : Fiji Islands, Tahiti.

T. Javanicum, $B l$. Hab.: Fiji and Samoan Islands. Hook. Gard. Ferns, t. 37.

T. meifolium, Bory. Hab.: Polynesian Isles; Oahu.

T. maximum, Blume. Hab. : Polynesian Islands.

T. giganteum, Bory. Hab.: Fiji Group.

T. gemmatum, J. Smith (T. cellulosum, Sturm; Hook. Second Cent. Ferns, t. 63). Hab. : Polynesian Islands.

T. longisetum, Bory. (T. Pluma, Hook. Cent. of Ferns, t. 97). Hab. : Samoan Isles.

\section{Davallia.}

D. parallela, Wall. Hab. : Sandwich Islands.

D. pectinata, Smith. Hab. : Tropical Polynesian Islands, Otaheite, Society Isles. Hook. \& Gr. Ic. Fil. t. 139. \$.

D. sessilifolia, Blume, Hab. : Fiji Islands.

D. alpina, Blume. (D. Belangeri, Bory.). Hab. : Polynesia.

D. botrychioides, Brack. Hab. : Fiji Islands.

D. affinis, Hook. Hab.: Polynesian Isles.

D. trichomanoides, Hook. Hab.: wooded mountains, Naviti Levu, Fiji Isles. Hook. Second Cent. Ferns, t. 64. \$. 
Davallia-continued.

D. pulchella, Hook. Hab. : Fiji and Samoa.

D. repens, Desv. Hab. : Polynesian Isles, Sandwich Isles.

D. contigua, Sw: Hab.: Pacific Isles, Otaheite. Hook. \& Gr. Ic. Fil. t. 141.

D. solida, Swz. Hab.: Pacific Islands; Tahiti, Friendly Isles, Samoan Isles, Pitcairn's Isle, Wallis' Island.

D. elegans, Swz. Hab.: Fiji Islands; Tahiti.

D. (Microlepia) strigosa, Swa. (D. Khasyana, Hook.). Hab.: Sandwich and Fiji Islands.

D. (Micro.) campyleura, Kunze (D. Amboynensis, Hook.). Hab.: Offack, Terre des Papons. D'Urville. \$.

D. (Micro.) Denhami, Hook. Hab. : Naviti Levu, Fiji Islands, and Sannoa. (Hook. Second C'ent. Ferns, t. 47.) $\$$.

D. (Micro.) hirta, Kaulf. Hab.: Sandwich Islands, Oahu.

D. (Micro.) Speluncæ, Baker (D. polypodioides, Hook.). Hab. . Polynesian Isles; Oahu.

D. (Micro.) Mannii, Eaton. Hab.: Hawaiian Islands. II. Mann.

D. gibberosa, Swarta. Hab. . Pacific Islands, Otaheite, Pitcairn's Island. \$.

D. fœeniculacea, Hook. Hab. . Naviti Levu, of the Fiji Islands. Hook. Second Cent. Ferns, t. 54. \$.

D. tenuifolia, Swartz. Hab.: Sandwich Islands.

\section{Cystopteris.}

C. fragilis, Bernh. (C. Douglasii, Hook.). Hab.: Sandwich Islands. Hook. Cent. Ferns, t. 55.

\section{Iindsaya.}

L. Lapeyrousii, Baker. Hab.: Island of Vaniholla or Pitt's Island, S. Pacific Ocean; Naviti Levu, Fiji Islands, damp places on mountains. Hook. Second Cent. Ferns, t. 56. \$.

L. trichomanoides, Dry. (L. Lessonii, Bory.). Hab. : Fiji Islands.

L. nitens, Blume. (L. recurvata, Wall.). Hab. : Polynesia.

L. ensifolia, Swo. Hab.: Fiji Islands. Hook. Gard. Ferns, t. 62.

L. pumila, Hook. MSSS. (non Klotzsch). Hab. . Sandwich Islands. $\$$.

L. erecta, Hook. MSS. Hab. : Sandwich Islands. \$.

L. falcata, Hook. MLSS. Hab. : Sandwich Islands. \$8.

\section{Adiantum.}

A. lunulatum, Burm. Hab. Samoan and Fiji Isles.

A. diaphanum, Blume. Hab.: Fiji.

A. fulvum, Raoul. Hab.: Fiji.

Isles.

A. Capillus-Veneris, L. Hab. : Polynesian Islands, Sandwich

A. hispidulum, Sw. Hab.: Fiji, Society Isles.

\section{Pellaea.}

P. geraniæfolia, Fée. Hab.: Fiji Islands; Owhyhee.

P. ternifolia, Fée. Hab.: Sandwich Islands; on Mlauna Loa and Mauna Kea, elev. 9,000 feet, in great luxuriance.

\section{Pteris.}

Pt. Iongifolia, L. Hab.: Pacific Isles, Fiji Isles.

Ht. cretica, $L$. Hab. : Fiji and Sandwich Islands.

Pt. crenata, Swartz. Hab.: S. Pacific Isles; Fiji and Samoa. 
Pteris-continued.

Pt. irregularis, Kaulf. Hab.: Sandwich Islands. \$.

Pt. excelsa, Gaud. Hab. : Sandwich Islands.

Pt. aquilina, $L$. Hab. . Sandwich Islands, Oahu, woods of the Society Islands.

Pt. rugulosa, Labill. Hab.: Otaheite, in mountain woods,rare. $\$$.

Pt. patens, Hook. Hab. : Society Islands.

Pt. decipiens, Hook. Hab.: Oahu, Sandwich Islands. \$.

Pt. decora, Bracken. Hab.: Sandwich Islands. \$\$.

Pt. comans, Forst. Hab.: Polynesian Islands; Society Isles, and Angau, Fiji.

Pt. incisa, Thunbg. (Pt. sinuata, Brack.). Hab.; Ovolau, Fiji Islands, in thickets at an altitude of $2,000 \mathrm{ft}$.

Pt. tripartita, Swartz, Hab.: Fiji Islands.

\section{Ceratopteris.}

C. thalictroides, Brong. Hab.: tropical and subtropical regions, in quiet waters; Ladrone Islands.

\section{Iomaria.}

L. Patersoni, Spreng. Hab.: Ovolau and Ingau (Fiji Group).

L. attenuata, Willd. Hab. . Orolau, Fiji Islands.

Islands.

L. vulcanica, Blume. Hab. : Fiji Islands, Ovolau; South Pacific

L. lanceolata, Spreng. Hab. : Society Islands.

L. procera, Spreng. Hab. : Society Islands, mountains of Tahiti;

Loyalty Islands; mountain of Gnau, Fiji Group.

L. filiformis, A. Cunn. Hab. : Sandalwood Bay, Fiji Isles.

\section{Blechnum.}

B. nitidum, Presl. Hab.: Marianne Islands, Guam.

B. orientale, Linn. Hab.: Pacific Isles, Fiji and adjacent islands, Fitzroy Islands, Coral Island, Tahiti.

\section{Sadleria.}

S. cyatheoides, Kaulf. Hab.: Sandwich Islands.

S. squarrosa, Gaud. Hab. : Sandwich Islands. \$.

\section{Doodfa.}

D. media, R. Br. (D. connexa, Kze.; D. Kunthiana, Gaud.). Hab.: Oahu, Ovolau, Fiji Islands; Pitcairn's Island, Otaheite.

\section{Asplenium.}

A. Nidus, L. (A. australasicum, Hook.). Hab. : Society Islands, Oahu, Sandwich Isles, Elizabeth Island, Fiji Isles.

A. Sundense, Blume. (A. vittæforme, J. Sm.). Hab.: Palapa, Marianne Islands, Angau, Naviti, Ovolau, Fiji.

A. Fejeense, Brack. Hab.: on trees and moist rocks, $F_{i j i}$ and Samoan, or Navigator's Islands.
A. serratum, Linn. Hab. : Society Isles.
A. fragile, Presl. Hab.: Sandwich Isles.
A. Trichomanes, $L$. Hab.: Sandwich Isles.
A. monanthemum, Linn. Hab. : Sandwich Isles.
A. multilineatum, Hook. Hab. : Samoa and Fiji. \$\$.
A. Sumatranum, Hook. Hab. . Fiji.
A. vulcanicum, Blume. Hab.: Fiji. 
Asplenium-continued.

A. tenerum, Forst. (A. elongatum, Sw.). Hab.: Samoa and Fiji, Marianne Islands, Tahiti.

A. erectum, Bory. Hab. : Sandwich Islands, rare.

A. contiguum, Kaulf. Hab. : Sandwich Islands; Oahu.

A. hirtum, Kaulf. Hab. : Ladrones; Guaban Island.

A. enatum, Brack. Hab.: Kaala Mountains, Oahu, Sandwich Islands. $\$$.

A. Kaulfussii, H. Mann. (A protensum, Kaulf.). Hab.: Oahu, Sandwich Isles. $\$$.

A. obtusatum, Forst. Hab. : Pacific Islands, Kermadee Group, Sandwich Islands.

A. gemmiferum, Schrad, Hab.: Sandwich Isles. H. Mann.

A. falcatum, Lam. Hab.: Friendly Islands, Sandwich Islands, Pitcairn's Island, Fiji Isles.

A. caudatum, Forst. Hab.: Pacific Isles, Sandwich Isles, Society Islands.

A. macrophyllum, Swartz. Hab.: Fiji Isles and Solomon's Group.

A. resectum, Smith. Hab.: Oahu and Fiji, Ovolau.

A. horridum, Kaulf. Hab. . Sandwich Islands, Oahu, Samoa.

A. Adiantum-nigrum, Linn. Hab.: Mauna Loa and other mountains, Sandwich Islands, 8-10,000 feet.

A. dissectum, Brack. Hab.: Sandwich Islands. $\$$.

A. cuneatum, Lam. Hab.: Sandwich Islands.

A. furcatum, Thunb. Hab.: Sandwich Islands, on the Kaala Mountains, Oahu.

A. affine, Swartz (A. spathulinum, J.Sm.). Hab.: Sandwich Islands, in forests ; Fiji Islands.

A. nitidum, Swoz. Hab. : Sandwich Isles. Mann.

A. laserpitiifolium, Lam. Hab. : Fiji Group of islands, abundant.

A. acuminatum, Hook. \& Arn. Hab. : Sandwich Isles, Oahu, abundant on the high mountains above Honolulu. \$.

A. rhizophyllum, Kunze (A. Macræi, Hook. \& Gr. t. 217). Hab. : Sandwich Isles and Fiji.

A. (Darea) bipinnatifidum, Brack. Hab. : Fiji. \$.

A. (Darea) flaccidum, Forst. Hab. : Sandwich Islands.

A. (Darea) rutæfolium, Kunze. Hab : Fiji.

A. (Darea) multifidum, Brack. Hab. . Tahiti, Society Islands and Ovolau; Fiji Islands, in damp forests. $\$$.

A. (Darea) Powellii, Baker. Hab. : Samoa. \$.

A. (Athyr.) grammitoides, Mook. Hab. : Sandwich Isles.

A. (Athyr.) deparioides, Brack. Hab.. Oahu. \$.

A. (Athyr.) (A. Poiretianum, Gaud.) aspidioides, Schlecht. Hab. : Sandwich Isles.

A. (Dipl.) sylvaticum, Presl. Hab.: Samoa.

A. (Dipl.) arborescens, Mett. Hab.: Polynesian Islands.

A. (Dipl.) melanocaulnn, Baker. Hab.: Fiji.

A. (Dipl.) Arnottii, Baker. Hab. : Sandwich Islands, frequent. \$\$.

A. (Dipl.) Sandwichianum, Mett. Hab. : Oahu, Sandwich Isles.

A. (Aniso.) decussatum, Swartz. Hab. : Fiji Islands.

A. (Aniso.) vitiense, Baker. Hab.: Fiji Islands. \$".

\section{Allantodia.}

A. Brunoniana, Wall. Hab. : Tahiti. 


\section{Gcolopendrium.}

S. Durvillei, Bory. Hab. : Ualau. \$.

\section{Didymochlaena.}

D. lunulata, Desv. Hab. : Fiji Islands. Hook, Gard. Ferns, t. 17.

\section{Aspidium.}

A. (Polyst.) aculeatum, Swz. Hab. : Sandwich Islands; on the western base of Mauna Loa, in woods in Kona, Hawaii, at an elevation of 5,000 or 6,000 feet. Horace Mann.

A. Haleakalense, Brack. Hab.: Sandwich Isles. Horace Mann.

A. (Polyst.) Richardi, Hook. Hab.: Fiji.

A. (Polyst.) capense, Willd. (A. coriaceum, Sw.). Hab. : Polynesian Islands.

A. (Polyst.) aristatum, Si. Hab.: Fiji, Samoa.

A. (Cyst.) falcatum, Swo. Hab. : Sandwich Isles.

\section{Nephrodium.}

Isles..

N. (Lastrea) patens, Desv. Hab. : Sandwich Isles, Oahu, Fiji

N. (Last.) albo-punctatum, Desv. Hab. : Fiji and adjacent isles.

N. (Last.) globuliferum, Hook. Hab.: Hawaii, Sandwich Islands, in dense forests. $\$$.

N. (Lastrea) Filix-mas, Rich. Hab.. Sandwich Islands, alt. 8-10,000 feet.

N. (Last.) stipitatum, Baker. Hab. : Ladrones. \$\$.

N. (Last.) glabrum, Baker. Hab.: Sandwich Isles. \$

N. (Last.) tenuifolium, Hook. Hab. . Fiji ; Ovolau, in mountain forests. $\$$

N. (Last.) rubiginosum, Hook. Hab. : Sandwich Islands. \$.

N. Fijiense, Hook. Second Cent. Ferns, t. 67. Hab.: Sandwich Islands. Horace Mann. \$\$.

N. (Last.) squamigerum, Hook. \& Arn. Hab. : Sandwich Isles. \$\$.

N. (Last.) decompositum, $R . B r$. Hab.. Tahiti ; Ngau, Fiji Islands.

N. (Last.) membranifolium, Presl. Hab.: Samoa.

N. (Last.) Milnei, Hook. Hab. : wooded mountains of Navitı Levu, Fiji Islands. Hook. Second Cent. t. 62. \$8.

N. (Last.) latifrons, Hook. Hab.: Oahu, Sandwich Islands, high mountains behind Honolulu. \$.

N. (Last.) setigerum, Baker (N. tenericaule, Hook.). Hab. : Society Islands.

N. arborescens, Baker. Hab. : Samoa. \$.

N. (Last.) davallioides, Baker. Hab.. Fiji, Tahiti, Samoa. \$.

N. unitum, $R . B r$. (non Sieb.; N. propinquum, Br.). Hab.: Sandwich Islands, abundant.

N. pteroides, $J$. Sm. Hab. : Samoa.

N. extensum, Hook. (N. Hudsonianum, Brack.). IIab.: Sandwich Isles.

N. dissectum, Desv. Hab.: Common in the Polynesian Islands. \$\$.

N. cucullatum, Baker (N. unitum, Sieb.). Hab. : Fiji.

N. cyatheoides, Kaulf. Hab. : Sandwich Islands, Oahu.

N. truncatum, Presl. Hab. : Pacific Isles.

N. (Pleo.) Leuzeanum, Hook. Hab. : Samoan and Fiji Islands. 
Nephrodium-continued."

N. (Sagen.) subtriphyllum, Baker. Hab.: Pacific Islands;

Frankland Islands, S. Pacific; Tahiti (Aspid., Hook.).

N. (Sagen.) latifolium, Baker. Hab. . Polynesian Isles.

N. (Sagen.) irregulare, Baker. Hab.: Polynesian Isles. \$\$.

N. (Sagen.) decurrens, Baker (Aspid-pteropus, Kze.). Hab.:

Pacific Isles, Orolau and Fiji.

N. (Sagen.) pachyphyllum, Baker (Aspid. grande, J. Sm.). Hab. : Fiji.

N. (Sagen.) cicutarium, Baker. Hab. : Sandwich Isles.

\section{Nephrolepis.}

N. exaltata, Schott. Hab. : Pacific Isles; Otaheite, and Pitcairn's Isle, Fiji, Oahu.

N. acuta, Presl. Hab.: Society Islands, Fiji Islands, and Frankland Islands, and Dunk Island.

N. ramosa, Baker (N. obliterata, Hook.). Hab.: Fiji and Samoan Islands, Society Islands.

N. altescandens, Baker (Polyp., Coll.). Hab. : Samoa.

\section{oleandra.}

O. neriiformis, Cav. Hab.: Fiji, Samoa.

\section{Polypodium.}

Isles. \$.

P. (Pheg.) unisorum, Baker. Hab.: Mount Rauai, Sandwich

P. (Pheg.) unidentatum, Hook. \& Arn. Hab.: Sandwich Islands, Oahu, Hawaii. $\$$.

P. (Pheg.) crinale, Hook, \& Arn. Hab. : Sandwich Isles, forests; Hawaii. \$.

Isles. $\$$.

P. (Pheg.) Hillebrandi, Hook. Hab.: Honolulu, Sandwich

P. (Pheg.) punctatum, Thunb. (P. rugulosum, Labill.). Hab.: Sandwich Isles.

P. (Pheg.) Sandvicense, Hook. \& Arn. Hab.: Sandwich and Society Isles. $\$$.

P. (Pheg.) Keraudrenianum, Gaud. Hab.: Sandwich Isles.

P. (Goniopteris) simplicifolium, Hook. (Nephrodium, Second Cent. Ferns, t. 19). Hab. : Fiji Islands.

P. (Goniopt.) costatum, Hook. Hab.: Society Isles, in damp hollows, alt. 1,000 feet. Fiji Islands. \$.

P. (Goniopt.) stegnogrammoides, Baker (P. Sand vicense, Hook.). Hab. : Sandwich Islands, near the crater of Kilanea. \$S.

P. (Dictyopteris) macrodon, Reinw. (P. Cumingianum, Hook.). Hab. : Samoan Islands, Fiji Islands.

P. Hookeri, Brack. Hab. : Sandwich Islands, Owhyhee, Fiji.

P. pseudo-grammitis, Gaud. Hab.: Sandwich Islands. \$.

P. ligulatum, Baker. Hab. . Fiji. \$\$.

P. Samoense, Baker. Hab.: Samoa. \$.

P. serrulatum, Mett. Hab. : Sandwich Isles. (Xiphopteris Jamesoni, Hook. Second Cent. Ferns, t. 14.)

P. subpinnatifidum, $B l$. Hab. : Sandwich Isles, on trees.

P. cucullatum, Nees. Hab.: Samoa.

P. sarmentosum, Brack. Hab. : Sandwich Islands, on rocks and decayed wood, frequent. 
Polypodiun-continued.

P. Adenophorus, Hook. et Arn. Hab.: Sandwich Islands.

P. decorum, Brack. Hab. : Tahiti and Sandwich Isles.

P. blechnoides, Hook. Hab. : Island of Raialea, Friendly Isles; mountains, Fiji Isles.

P. pellucidum, Kaulf. Hab.: Oahu, Sandwich Isles. \$. Hook. Cent. Ferns, t. 44 and 45.

P. hymenophylloides, Kaulf. Hab.: Sandwich Isles. (Adenophorus, Hook. \& Gr. Ic. Fil. t. 176.)

P. tamariscinum, Kaulf. Hab. : Sandwich Isles, Tahiti. (Adenophorus, Hook. \& Gr. İc. Fil. t. 175.)

P. (Adenophorus) abietinum, Eaton. Hab.: Hawaii; Oahu, on trees 3,000 feet above the sea. H. Mann.

P. subauriculatum, Blume. Hab.: Fiji and Samoa.

P. serpens, Forst. (P. rupestre, $R, B r$.). Hab. : Society Isles.

P. (Niphobolus) adnascens, Sw. Hab.: Fiji.

P. (Phymatodes) accedens, Blume. Hab. : Samoan or Navigator's Islands, and Tahiti, Fiji Islands.

P. (Phym.) Brownii, Wickst. (P. attenuatum, $R . B r$.). Hab.: Fiji Islands.

P. (Phym.) lanceolatum, Linn. (P. lepidotum, Willd.). Hab.: Sandwich Islands.

P. (Phym.) angustatum, $S w$. Hab.: Pitcairn's Isle, and Tahiti.

P. (Phym.) lycopodioides, Linn. Hab.: Sandwich Isles.

P. (Phym. irioides, Lam. Hab. : Fiji Islands, Pitcairn's Isle.

P. (Phym.) Spectrum, Kaulf. Hab. : Sandwich Isles.

P. (Dipteris) Dipteris, Blume. (P. Horsfieldii, R. Br.). Hab.: Fiji, Ovolau.

P. (Phym.) phymatodes, Linn. Hab.: Pacific Islands, most abundant; Fiji, Samoa. Isles.

P. (Phym.) nigrescens, Blume. Hab.: Fiji, Samoa, Friendly

P. (Phym.) Powellii, Baker. Hab.: Samoa. \$.

P. (Phym.) dilatatum, Wall. Hab.: Samoa or Navigator's Islands.

P. (Phym.) alatum, Hook. Hab. : Fiji.

P. (Drynaria) quercifolium, Linn. Hab. : Fiji.

P. (Ury.) rigidulum, Sw. (P. diversifolium, $R$. Br.). Hab. : Fiji.

Nothochlaena.

N. hirsuta, Desv. Hab. : Society Islands, Fiji, Ovolau.

\section{Monogramme.}

M. Jungbuhnii, Hook. Hab. : Isle of Ovolau, Samoan and Fiji Islands, Sandwich Islands, Society Islands.

\section{cymnogramme.}

G. Javanica, Blume. Hab. : Sandwich Islands, Fiji.

G. (Ceropteris) calomelanos, Kaulf. Hab.: Samoa.

G. (Syngramme) quinata, Hook. Hab.: Island of Vanecolla or Pitt's Island, South Pacific.

G. (Selliguea) lanceolata, Hook. Hab.: Fiji, Samor.

G. (Sell.) candiformis, Hook. Hab. : Tahiti, Fiji Isles.

G. (Sell.) pinnata, Hook. Hab. : Fiji, Orolau. 


\section{Antrophyum.}

A. subfalcatum, Brack. (A. Brookei, Hook. Second Cent. Ferns, t. 79). Hab. : Fiji Islands, Samoa.

A. plantagineum, Kaulf. Ilab.: Marianne Islands, Society Islands; Tahiti.

A. reticulatum, Kaulf. Hab.: Aneiteum.

A. semicostatum, Blume. Hab.: Pacific Islands, abundant ; Society Islands and Solomon's group; Fiji, Aneiteum, Navigator's Islands.

\section{Vittasia.}

V. elongata, Sw. Hab.: Sandwich Islands, Graham, Marianne

Islands.

\section{Femionitis.}

H. lanceolata, Hook. Hab.: Fiji. Hook. Second Cent. t. 55. \$.

\section{Acrostichum.}

A. microdenium, Fee. Hab. : Sandwich Isles.

A. conforme, Sw. Hab.: Sandwich Isles, Samoa, Fiji.

A. Samoense, Baker. Hab.: Polynesian Islands. \$.

A. squamosum, Sw. Hab.: Sandwich Islands.

A. (Stenochlæna) sorbifolium, Limn. Hab.: Fiji, Samoa.

A. (Steno.) scandens, $J$. Sm. Hab.: Fiji.

A. (Polybotrya) Wilkesianum, Hook. Hab. : Society Isles.

A. (Aconiopteris) gorgoneum, Kaulf. Hab.: Sandwich and Society Isles. \$.

A. (Gymnopteris) repandum, Blume. Hab. : Polynesian Isles.

A. (Chrysodium) reticulatum, Kaulf. Hab. : Sandwich Isles. \$.

A. (Chrys.) Blumeanum, Hook. Hab.: Samoa and Navigator's Islands, abundant, but local, in damp shady places, running orer rocks and up high trees.

A. (Chrys.) aureum, Linn. Hab.: Polynesia, Fiji, Society and Samoan Islands.

A. (Chrys.) polyphyllum, Hook. Hab. : Fiji.

A. (Hymenolepis) spicatum, Linn. Hab. : Society Isles.

\section{Schizaea.}

S. australis, Gaud. Hab.: Sandwich Isles. H. Mann.

S. robusta, Baker (S. australis, Brack.). Hab.: Sandwich Isles. \$.

S. (Lophidium) dichotoma, Sw. Hab. : Polynesia.

S. (Actinostachys) digitata, Sw. Hab.: Fiji.

\section{Iygodium.}

L. semihastatum, Desv. Hab.: Marianne Isles.

L. (Hydroglossum) reticulatum, Schk, Hab. : Polynesian Isles.

\section{Angiopteris.}

Manattia.

A. evecta, Hoffm. Hab.: Society Islands.

M. fraxinea, Smith. Hab.: Polynesia.

M. (Gymnotheca) Douglasii, Baker. Hab.: Sandwich Isles. \$.

\section{Ophioglossum.}

O. nudicanle, L. fil. (O. ellipticum, IIook. \& Gr. t. 40). Hab. . Sandwich Isles. Horace Mam.

O. vulgatum, Limn. Hab.: Sandwich Isles.

O, reticulatum, Linn. IIab. : Polynesia.

O. (Ophioderma) pendulum, $L$. Hab. : Polynesian Isles.

\section{Botrychium.}

B. daucifolium, Wrall. Hab. : Sandwich and Society Isles. 


\section{AFRICA.}

1. TROPICAL AFRICA AND ISLANDS.

2. CAPE COLONY, NATAL, TRISTAN D'ACUNHA.

$\$$ denotes the only hitherto known localities.

\section{TROPICAL AFRICA AND ISLANDS, INCLUDING} ABYSSINIA AND ARABIA.

\section{Gleichenta.}

G. polypodioides, Sm. Hab.: Tropical Africa, north to Angola. G. Boryi, Kze. Hab. : Bourbon. \$.

G. Hlagellaris, Spreng. Hab.: Bourbon, Mauritius, Madagascar.

G. dichotoma, Willd. Hab.: Bourbon, Mauritius, Seychelle Islands, Madagascar, Comoro Isles, Mayotta; Fernando Po (long narrow segm.), Island of St. Thomas, Sierra Leone; Cameroon Mountains, Congo.

\section{Cyathea.}

C. Manniana, Hook. Hab.: Fernando Po, and Cameroon Mountains, alt. $3-4,000$ feet. $\$$.

C. Welwitschii, Hook. Hab.: W. Africa, Island of St. Thomas, in the Bight of Biafra, alt. 3-7,000 feet; Fernando Po, Angola. \$.

C. Camerooniana, Hook. Hab. : Cameroon Mountains, alt. 34,000 feet. \$.

C. Angolensis, Welvo. Hab.: Benguela, district of Huilla, Tropical W. Africa, alt. 3,500-5,550 feet; very rare. Welwitsch. \$.

C. Kirkii, Hook. Hab. : Comoro Isles, Johanna Island, alt. 2,000 feet. Dr: Kirk. \$s.

C. Seychellarum, Mett. Hab.: Seychelles. \$.

C. excelsa, Swz. Hab. : Bourbon and Mauritius. \$.

C. canaliculata, Willd. Hab.: Bourbon and Mauritius, Madagascar. $\$ 5$.

C. glauca, Bory. Hab. : Bourbon.

\section{Eemitelia.}

H. Capensis, $R . B r$. Hab. : Mauritius, Comoro Isles. Kuhn.

H. Junghuniana, Mett. Hab. : Antananarivo, Madagascar (?).

\section{Alsophila.}

A. Athiopica, Welw. MS. Hook. Hab.: Tropical Africa, S. of the Line; Angola; Golungo alto, alt, 1-2,400 feet.-Welwitsch. Comoro Isles, Johanna Island.

A. obtusiloba, Hook. Hab. . Sierra del Crystal, Tropical Africa. 


\section{Dicksonia.}

D. arborescens, L'Hérit. Hab.: Island of St. Helena, only near the highest summit of Diana's Peak. J. D. Hooker. \$.

D. abrupta, Bory. Hab. : Bourbon, amongst scoriæ and lava. \$.

D. rubiginosa, Karlf. ( $\beta$ D. anthriscifolia, Kaulf.). Hab.: Bourbon and Mauritius.

\section{Eymenophyllum.}

H. rarum, $R . B r$. Hab. : Bourbon and Mauritius, Comoro Isles. Hook. \& Gr. Ic. Fil. t. 33.

H. capillaceum, Roxb. Hab. . St. Helena, on rocks and treeferns; Diana's Peak. \$.

H. gracile, Bory. Hab.: Bourbon and Mauritius, Madagascar. Hook. \& Gr. Ic. Fil. t. 198.

H. polyanthos, Swz. Hab.: Mauritius and Madagascar; W. Tropical Africa.

H. ciliatum, Swz. (H. Boryanum, Willd.). Hab. : West Tropical Africa, Fernando Po, Cameroon Mountains, \&c. ; and in the Fast, in the Zambesi district, Madagascar, Seychelles, Comoro Isles, Mayotta and Johanna Island, Bourbon, Mauritius. Hook. \& Gr. Ic. Fil. t. 35 ; and H. Plumieri, Hook. \& Gr. Ic. Fil. t. 123.

H. elasticum, Bory. Hab. : Bourbon and Mauritius, Madagascar and Seychelles. Hook. \& Gr. Ic. Fil. t. 135. \$.

H. lineare, Swz. Hab. : Mauritius. H. trifdum, Hook. \& Gr. Ic. Fil. t. 196.

H. Tunbridgense, Smith. (H. unilaterale, Bory.). Hab. : Bourbon.

H. triangulare, Baker. Hab.: Fernando Po. \$.

\section{Trichomanes.}

T. cuspidatum, Willd. Hab. : Bourbon and Mauritius, Madagascar, Comoro Isles, Seychelles. Kuhn; Hook. \& Gr. Ic. Fil. t. 155. $\$$.

T. Barklianum, Baker. Hab.: Tamari Cascade, Mauritius. \$.

T. muscoides, Suz. (T. erosum, Willd.). Hab. : West Africa, by the river Niger; Fernando Po ; Prince's Island (var. Lenormandi, V. D. Bosch.), Comoro Isles, Mayotta, Johanna Island. Kuhn.

T. parvulum, Poiret. Hab.: Bourbon, Madagascar, Johanna Island.

T. Mannii, Hook. MSS. Hab.: Fernando Po. \$.

T. Alabellatum, Bory. (non V. D. B.). Hab.: Bourbon and Mauritius.

T. digitatum, Sw: Hab.: Bourbon and Mauritius.

T. Kirkii, Hook. MSS. Hab.: Johanna Island and West Africa. \$.

T. Filicula, Bory. (T. dilatatum, T. D.Bosch.). Hab.: Bourbon and Mauritius, Madagascar, Comoro Isles, West Africa, the River Nun, Fernando Po. $\Pi u h r$.

T. pyxidiferum, $L$. Hab.: Fernando Po, Angola, Bourbon, Mauritius. Hook. \& Gr. Ic. fll. t. 206.

T. radicans, Swz. (T. speciosum, Willd.). Hab. : Tropical West Africa, Island of St. Thomas, Fernando Po, Angola (var. gigantea, Mett.), Johanna Island, Comoro Isles, Bourbon. $\boldsymbol{K} u h r$.

T. crispum, L. Hab. : West Tropical Africa, banks of the Nun and the Niger ; Brass River, and Prince's Island (in Baikie's Niger Expedition, Hook. Gard. Ferns, t. 27). 
Trichomanes-continued.

T. Javanicum, Blume, Hab.: Madagascar.

T. rigidum, Swz. (T. Boivini, V.D.B.). Hab. : Angola, Fernando $\mathrm{Po}$, Bourbon, Mauritius ; St. Mary's, near Madagascar; Comoro Isles, Mayotta. Kuhn.

T. giganteum, Bory. Hab.: Mauritius ; Bourbon, in one wood at the great lake; Johanna Island.

T. longisetum, Bory. (T. Pluma, Hook. Cent. Ferns, t. 97). Hab. . Bourbon.

T. fœeniculaceum, Bory. Hab. : Bourbon, Mauritius, Madagascar.

\section{Davallia.}

D. pedata, Smith. Hab. Bourbon and Mauritius.

D. repens, Desv. (D. Boryana, Presl.). Hab. . on trunks of trees; Bourbon, Mauritius, Seychelles.

D. elegans, Swz. Hab.: Madagascar, Comoro Isles; Johanna Island, Mayotta, Seychelles; Angola, Fernando Po.

D. Mauritiana. Hab.: Mauritius, Johanna Island. $\$$.

D, nitidula, Kunze. Hab. : Angola, at 1-2,000 feet.

1. Speluncæ, Baker (D. polypodioides, Hook.). Hab.: Fernando Po, Senegambia, Guinea, Congo ; Bourbon, Mauritius, Madagascar, Seychelles.

D. concinna, Schrad. (D. Schimperi, Hook.). Hab.: Angola, Fernando Po, Bourbon; trunks of trees near Adesula, Abyssinia. Schimper.

D. nigrescens, Hook. Hab. : Peak of Fernando Po, at an elevation of 3,000 feet. Hook. Second Cent. Ferns, t. $93 . \$$. \$.

D. Gondotiana, Kunze. Hab.: Madagascar, at Emirne. \$.

D. tenuifolia, Swz. Hab.: Bourbon, Mauritius, Madagascar, Comoro Isles, Mayotte.

D. Melleri, Hook. MSS. Hab. : Madagascar. \$.

\section{Cystopteris.}

C. fragilis, Bernh. Hab. . Fernando Po; Cape de Verde Island, Fogo Island; Bourbon, Mauritius; Abyssinia.

\section{Iindsaya.}

L. cultrata, Swz. Hab.: Bourbon, Madagascar.

L. Kirkii, Hook. MSS. Hab.: Seychelles, on the hills. Kuhn. \$.

L. Irnuginosa, Wall. Hab.: Bourbon and Mauritius, gathered at the mouth of the Kongone River, Livingston Expedition.

L. ensifolia, Swz. Hab. : Mauritius, Madagascar, Isle of Nissobi (or Nossi-beh), west coast of Tropical Africa, in the Nun River. Hook. Gard. Ferns, t. 62.

L. heterophylla, Dry. (L. cuneata, Willd.). Hab. : Bourbon, Mauritius.

\section{Adiantum.}

A. reniforme, $L$. ( $\beta$ A. asarifolium, Willd.). Hab.: Bourbon and Mauritius, growing on the mountains and in arid regions. Senegambia (?). Kuhn.

A. lunulatum, Burm. Hab.: Madagascar, Island of Nossi-beh, Comoro Isles, Johanna Island, Zambesi Land; Angola, Quorra River, Guinea, Cape de Verde Islands.

A. caudatum, Linn. (A. hirsutum, Bory.). Hab.: Bourbon, II 2 
Adiantum-continued.

Mauritius, Madagascar, Comoro Isles, Johanna Island ; near Kitandam, in the Island Angasilia.-Kuhn. Seychelles; Angola, Congo, banlrs of the Niger; Cape de Verde Islands; Arabia.

A. tetraphyllum, Willd. (A. fructuosum, Spr. ; A. prionophyllum, H. B. K.). Hab.: Fernando Po, Congo.

A. Capillus-Veneris, L. Hab.: Niger Valley, Angola, Bourbon, Mauritius, Madagascar; Nossi-beh, Zambesi Land; Abyssinia; Arabia.

A. Athiopicum, L. Hab. : Cameroon Mountains, at 7,000 feet; Zambesi Land, Bourbon, Mauritius, Madagascar.

A. hispidulum, Swz. Hab.: Bourbon, Mauritius, Comoro Isles, Angasilia, Zambesi Land; banks of the Niger.

\section{ochropteris.}

O. pallens, J. Smith. Hab. : Bourbon, Mauritius, Madagascar.

\section{ronchitis.}

L. pubescens, Willd. Hab.: Bourbon, Mauritius, Madagascar, Nossi-beh Island, Johanna Island, Seychelles; and in West Tropical Africa, on the Cameroon Mountains, and the Islands of Fernando Po, and St. Thomas.

L. occidentalis, Baker. Hab.: Angola, Fernando Po, at an elevation of 4,000 feet on the Cameroon Mountains. \$.

\section{Eypolepis.}

Po. \$.

H. anthriscifolia, Presl. Hab.: Bourbon, Mauritius, Fernando

H. Bergiana, Hook. Hab. : Zambesi Land.

\section{Chellanthes.}

Ch. (Adiantopsis) pteroides, Swo. Hab.: Tropical Eastern Africa, near Mombus. Kuhn.

Ch. (Adiant.) Kirkii, Hook. Hab. : gathered in Zambesi Land, on rocks in the Moramballa Mountains, at an elevation of from 2-3,500 feet; and in Angola. Hook. Second Cent. Ferns, t. 81. \$.

Ch. (Adiant.) Schimperi, Hook. Hab.: Abyssinia. Kuhn. \$.

Ch. arabica, Decaisne. Hab.: Abyssinia, near Djel. Kuhn. \$.

Ch. hirta, Swz. Hab.: Angola.

Ch. multifida, Swoz. Hab.: Zambesi Land, St. Helena.

Ch. Welwitschii, Hook. MSS. Hab. : Angola, frequent, 2,4003,800 feet. $\$$.

Ch. farinosa, Kaulf. Hab.: Cameroon Mountains, Angola, Zambesi Land; Bourbon, Comoro Isles; Abyssinia; Arabia. Kuhn; Hook. \& Gr. Ic. Fil. t. 134.

\section{Onychium.} t. 2.

O. melanolepis, Decaisne. Hab.: Abyssinia. Hook. Cent. Ferns,

\section{Pellza.}

P. pilosa, Hook. Hab. : Bourbon. \$

P. geraniæfolia, Fée. Hab.: Bourbon and Mauritius, Madagascar, Zambesi Land, Angola.

P. dura, Hook. Hab.: Bouxbon, Mauritius, Madagascar, Nossibeh; Comoro Isles, Angasilia; and recently discovered in Angola, at $3,800-5,500$ feet. $\$$. 
Pellea-continued.

P. Boivini, Hook. Hab.: Mauritius, Madagascar, Nossi-beh, Comoro Islands.

P. consobrina, Hook. Hab. : Madagascar, Cameroon Mountains. Kuhn.

P. Barklyæ, Baker. Hab.: Seychelles. $\$$.

P. Doniana, Hook. Hab.: Tropical Africa, the Niger country, Angola ; Zambesi Land. \$\$.

P. hastata, Link. Hab.: Bourbon, Mauritius, Madagascar, Comoro Islands, Johanna Island, Nossi-beh; burning mountain, Angasilia Island, Seychelles, Angola, Cameroons; Cape Verde Islands, Island St. Nicholas.-Kuhn. Zambesi Land.

P. calomelanos, Link. Hab.: Zambesi Land, Angola.

P. articulata, Balker (Pteris, Kaulfs.). Hab.: Bourbon and Mauritius, arid rocky places ; cascade of Reduit, and that of Tamarin; Madagascar, rocks on the ground of the Chute d'Eau d'Ouwilave Rivière Divoudron. \$\$.

\section{Pteris.}

Pt. longifolia, $L$. Hab. : Isle San Nicol, Cape Verde Islands, Angola; Bourbon, Mauritius, Madagascar, Comoro Isles, Mayotte, Zanguebar, Mombas; Abyssinia. Kuhn.

Pt. cretica, $L$. Hab.: Bourbon, Mauritius, Abyssinia, Arabia.

Pt. pellucida, Presl. Hab. : Guinea coast.

Pt. Madagascarica, Ag. Hab.: Madagascar, Comoro Isles, Isle Mohil. \$.

Pt. quadriaurita, Retz. Hab.: West Tropical Africa, Senegambia, Fernando Po, Angola, Zambesi Land, Madagascar.

Pt. scabra, Bory. Hab.: woods in Mauritius and Bourbon, Madagascar. $\$$.

Pt. paleacea, Roxb. Hab. : summit of Diana's Peak in the upper exposed part of $\mathrm{St}$. Helena. \$\$.

Pt. arguta, Ait. Hab. : St. Helena. Kuhn.

Pt. flabellata, Thurb. Hab.: Bourbon, Mauritius, Fernando Po, Isle of St. Thomas, Angola, Ascension Island; Green Mountain, 12-1,800 feet elevation; St. Helena, southern declivity of Diana's Peak; Cape Verde Islands; East Tropical Africa, on Mount Kilimandjaro, region of Dschagga, 5,800-7,800 feet. $-K u h n$. Abyssinia, by streams in mountain districts near Sabra.

Pt. brevisora, Baker (Hppolepis Pterioides, Hook.). Hab.: Cameroon Mountaius, and Fernando $P_{0}$, at an elevation of 4-7,000 feet. Hook. Second C'ent. Ferns, t. 59. \$\$.

Pt. aquilina, $L$. Hab. : Cape de Verde Islands, Fernando Po, Sierra Leone; Cameroon Mountains, to 7,000 feet; Bourbon, Mauritius, Madagascar, Comoro Isles, Island of Angasilia, Mount Kilimandjaro.-Kuhn. A byssinia, 8-9,000 feet, near Genansa.

Pt. biaurita, L. Hab.: Bourbon, Mauritius, Comoro Isles, Johanna Island, Mayotte, Angasilia.-Kuhn. Fernando Po, Congo, Sierra Leone, river Niger, Cameroon Mountains.

Pt. triplicata, Agardh, Hab.: Mozambique Channel, Madagascar. \$.

Pt. Melleri, Baker. Hab. . cascades of the Anevoca Valley, Madagascar. \$

Pt. Lancæfolia, Agardh. Hab. : interior of Madagascar. \$. 
Pteris-continued.

Pt. Mannii, Baker. Hab.: Fernando Po. \$.

Pt. Laurea, Desv. (Pt. triphylla, Ag.). Hab.: Madagascar. \$.

Pt. Currori, Hook. Hab.: West Tropical Africa, at the Niger River, near Elephant's Bay, and on the mountains of Fernando Po, at 3-500 feet. $\$ \$ \$$.

Pt. spinulifera, Schum. (Pt. atrovirens, W.). Hab. : Guinea coast and Angola, Sierra Leone, Promontory Palmas, Fernando Po; Prince's Island; Kingdom of Oware; Gulf Ambas, River Bagroo, Congo. Kuhu.

Pt. Woodwardioides, Bory. Hab.: Bourbon and Mauritius. \$.

Pt. incisa, Thunbg. Hab.: Buurbon and Mauritius, Ascension Island, covering many acres of ground on the weather slopes of Green Mountain, alt. 1,200 to 1,800 feet.-J. D. Hooker. Cameroons.

Pt. tripartita, Sizz. Hab.: Bourbon, Mauritius, Madagascar, Comoro Isles, Mayotte, Island Galega, Seychelles; West Tropical Africa; Fernando Po.

\section{ceratopteris.}

C. thalictroides, Brong. Hab. : tropical and subtropical regions, in quiet waters; Madagascar, turfy and marshy places; Senegambia, Guinea, Prince's Island.

\section{Iomaria.}

L. attenuata, Willd. Hab.: Bourbon, Mauritius, Madagasear, Fernando Po.

L. Boryana, Nilld. (L. Magellanica, Desv.). Hab.: Bourbon, Mauritius, Madagascar. Hook. Gard. Ferns, t. 52.

\section{Blechnum.}

B. anstrale, Linn. Hab. . Bourbon, Madagascar, Cape Verde Islands.

\section{Asplenium.}

A. Nidus, L. Hab.: Bourbon, Mauritius, Madagascar, Comoro Isles, Johanna Island, Seychelles, Galega Isle, Monbas.

A. sinuatum, Beauv. Hab.: Guinea coast, southward to Angola ; Oware, River Nun, Isle of St. Thomas; Fernando Po, on oil palms. $\$$.

A. Currori, Hook. Hab.: Guinea Coast, Elephant Bay, River Niger, Fernando Po. \$.

A. coriaceum, Baker. Hab.: Cameronn Mlountains. \$.

A. Gautieri, Hook. Hab.: Island of Nossi-beh. \$. Po. \$.

A. variabile, Hook. Hab. : on trees in gloomy forests, Fernaudo

A. alternans, Wall. Hab.: Abyssinia, near Aman Eski.

A. vagans, Baker. Hab. . Island of St. Thomas, West Tropical Africa; Madagascar. $\$$.

A. Sandersoni, Hook. Hab.: Zambesi Land, Johanna Island, Madagasear, Bourbon, Mount Kilimandjaro, district of Dschagga, 5,500-7,800 feet. Kuhn.

A. pygmæum, Hook. Hab. : Madagascar. \$.

A. monanthemum, $L$. IIab.: Cape Verde Islands, Fernando Po; Abyssinia, near Adesoula.

A. subarenium, Hook. Hab.: Madagascar 
Asplenium-continued.

\section{Garcia. \\ A. Iongissimum, Blume. Hab.: Mauritius, Island of Diego}

A. longicauda, Hook. Hab.: Cameroon Mountains, Prince's Island; Fernando Po, on trees; Peak Mountain, at an elevation above the sea of 3,000 feet. Hook. Second Cent. Ferns, t. $69 . \$$.

A. emarginatum, Beauv. Hab.: Tropical Western Africa, Guinea Coast and Angola; mountains of Prince's Island, Bight of Benin, river Onitoba; Fernando $\mathrm{Po}$, on mountains of 1,000 feet elevation. Hook. Second Cent. Fernss, t. 80 . \$.

A. tenerum, Forst. Hab. : Fernando Po, Seychelles. Kuhn.

A. lineatum, $S w z$. Hab.: Bourbon, Mauritius. \$.

A. erectum, Bory. Hab. . Western Tropical Africa, Prince's Island, Bight of Biafra, Fernando Po, Sugar-loaf Mountain; Sierra Leone, rare; Ascension Island; Green Mountain, in crevices of rocks most abundant, 1,000 feet elevation; Bourbon; Mauritius, common in moist shady rocky localities (var. proliferum). St. Helena, moist rocks, shady places; Diana's Peak, elevation 2,000 feet. (A. Baxteri, Hook. Second Cent. Ferns, t. 75.)

A. Prionitis, Kunze. Hab.: Angola, Bourbon, Mauritius.

A. anisophyllum, Kunze. Hab. : Bourbon, Zambesi Land ; Mount Kilimandjaro, 5,500-7,800 feet.-Kuhn. Cameroon Mountains.

A. hirtum, Kaulf. Hab.. Bourbon, Mauritius, Madagascar, Seychelles.

A. compressum, Swz. Hab. : St. Helena, 'exclusively, we believe, a native of St. Helena.' Hook. Sp. Fil. \$.

A. nitens, Swz. Hab.: Bourbon, Mauritius, Comoro Isles, Angasilia Isle. Kuhn.

A. platybasis, Kunze. Hab.: St. Helena. $\$$.

A. Serra, Langs \& Fisch. Hab.: Cameroon Mountains, Fernando Po.

A. marinum, Linn. Hab. : St. Helena. Kuhn.

A. gemmiferum, Schrad. Hab.: Angola, Zambesi Land, Bourbon, Mauritius, Madagascal.

A. auritum, Swz. Hab.: Bourbon.

A. falcatum, Lam. Hab.: Mascaren Isles, Zambesi Land.

A. caudatum, Forst. Hab. : Johanna Isle, Angola.

A. dimidiatum, Swz. Hab.: Tropical Africa; Fernando Po, growing on oil-palms.

A. macrophyllum, Swz. Hab.: Bourbon, Mauritius, Comoro Isles, Johanna Island, Mayotte; Nossi-beh, near Madagascar; Angasilia Island, near the city of Kitanda.

A. formosum, Willd. Hab. : Angola and Congo.

A. resectum, Sinith. Hab.: Bourbon, Mauritius, Seychelles, Angola and Guinea Coast, Fernando Po. Hook. \& Gr. Ic. Fil. t. 114.

A. protensum, Schrad. Hab. : Mauritius, Fernando Po; on Mount Kilimandjaro, district of Dschagga, 5,500-7,800 feet.-Kuhn, Abyssinia, mountains near Adesoula.

A. bipartitum, Bory. Hab.: Bourbon and Mauritius, in shady woods and on trunks of trees; Comoro Isles, Nossi-beh Island, Madagascar. $\$$. 
Asplenium-continued.

A. pumilum, Swo. Hab.: Zambesi Land; Abyssinia, rocky shady places on mountains near A mba Sea, alt. 6,000 feet.

A. Adiantum-nigrum, Linn. Hab.: Cape Verde Islands ; Cameroon Mountains, St. Helena, Bourbon and Mauritius, Abyssinia.

A. cuneatum, Lam. Hab. . Mozambique, Johanna Island ; Seychelles.

A. furcatum, Thumb. (Aspl, præmorsum; Swzz.). Hab. . Bourbon, Mauritius, Madagascar, Comoro Isles; Island of Angasilia, near Kitanda Mdjini.-Kuhn. Cameroons, St. Helena, Cape Verde Islands, A byssinia.

A. affine, Sw: Hab.: Bourbon, Mauritiue.

A. laserpitiifolium, Lam. Hab.: Praslin Isle, Seychelles.

A. nigritianum, Hook. Hab.: Prince's Island and St. Thomas; Guinea Coast, River Niger, Fernando Po. $\$ \$$.

A. lanceolatum, Huds. Hab.: St. Helena.

A. bulbiferum, Forst. Hab.: Bourbon, Seychelles, Johanna Island.

A. cicutarium, Swz. Hab.: Cameroon Mountains, Guinea Coast, Fernando Po ; shady places, middle region of Mount Silke, Abyssinia.

A. (Darea) Mannii, Hook. Hab.: Cameroon Mountains, and Peak of Fernando Po, elevation 3,000 feet above the level of the sea, epiphytal. Hook. Second Cent. Ferns, t. 60 . \$\$.

A. (Darea) brachypteron, Kunze. Hab.: Madagascar, Angola, Guinea Coast; Sierra Leone.

A. (Darea) irregulare, Baker, Hab.: Sierra del Crystal, Guinea Coast. $\$$.

A. (Darea) rutæfolium, Kunze. Hab.: Bourbon, Mauritius, Zambesi Land.

A. (Darea) Borbonicum, Hook. Hab. : Bourbon and Mauritius. \$.

A. (Darea) viviparum, Presl. Hab.: Bourbon and Mauritius, growing in all forest grounds on dead and decaying trees, among stones on the slopes of mountains, and in ravines and bottoms of water-courses. Hook. Fil. Exot. t. 64. \$.

A. (Athyr.) Filix-foemiua, Bernh. Hab.: Cameroon Mountains, 7,000 feet; Abyssinia.

A. (Athyr.) aspidioides, Schlecht. Hab. : Bourbon, Madagascar, Fernando Po.

A. (Athyr.) umbrosum, J. Sm. Hab.. Guinea Coast; Cape Verde Islands.

A. (Dipl.) humile, Baker. Hab.: Mauritius. \$\$.

A. (Dipl.) sylvaticum, Presl. Hab. . Mauritius, near Mombas; Fernando Po.

A. (Dipl.) Welwitschii, Hook. MSS. Hab. : Angola, \$.

A. (Dipl.) nigro-palenceum, Baker. Hab. : St. Helena. \$.

A. (Dipl.) arborescens, Mett. Hab.: Bourbon, Mauritius, Comoro Islands, Johanna Island.

A. (Aniso.) decussatum, Swz. Hab.: Bourbon and Mauritius, abundant; W. Tropical Africa, Fernando Po, Angola, and Guinea Coast.

A. (Hemidictyum) Ceterach, L. Hab. : Cape Verde Islands. Kuhn. 


\section{Actinlopteris.}

A. radiata, Link. Hab. : Bourbon, Mauritius, Madagascar, Zambesi Land, Zanguebar, kingdom of Madi, at the White Nile, Angola, Cape de Verdes, Island of Fogo; Corofan, Abyssinia; Arabia. Hook. Cent. Ferns, t. 75, 76.

\section{Didymochlaena.}

D. lunulata, Desv. Hab.: Madagascar, Comoro Isles, Johanna Island, Mayotte; Fernando Po, elevation of 4,000 feet, Peak mountains ; Island of St. Thomas; Cameroon Mountains. Hook. Gard. Ferns, t. 17.

\section{Aspidium.}

A. (Polyst.) aculeatum, Suz. Hab.: Fernando Po, on the Peak, elevation 9,000 feet; Bourbon and Muritius; (var. angulare) Comoro Isles; Angasilia Island, volcano, 6-9,000 feet.-Kuhn. Mount Silke, Abyssinia.

A. (Polyst.) Capense, $\Pi^{\top} i l l d$ (A. coriaceum, Swz.). Hab. : Bourbon, Mauritius, Madagascar.

\section{Nephrodium.}

N. (Lastrea) subbiauritum, Hook. Hab.: Bourbon. \$.

N. (Last.) punctulatum, Baker. Hab.: Cameroon Mountains, 4,500 feet. $\$$.

N. (Last.) patens, Desv. Hab.: St. Helena, Zambesi Land, Angola.

N. (Last.) Spekei, Baker. Hab. : Johanna Island, Angola. \$.

N. (Last.) crinibulbun, Hook. Hab.: west const of Africa, on mountain 2,000 feet elevation, Island of St. Thomas; E. Tropical Africa, near Mombas. Kuhn. \$s.

N. (Last.) albo-punctatum, Desv. Hab. : Guinea Coast, Sierra Leone, rscent of the Quorra, on a rock below the waterfall in Leicester Mountain, rare; Fernando Po; Bourbon and Mauritius, frequent; Comoro Isles.

N. (Last.) crinitum, Desv. Hab. : Mauritius, abundant; Bourbon. \$.

N. (Last.) conterminum, Desn. Hab. : Bourbon.

N. (Last.) prolixum, Baker (N. ochthodes, Hook.; N. appendiculatum, Hook.). Hab.: Mauritius and Bourbon.

N. (Last.) tomentosum, Baker (N. thelypteroides, Hook.). Hab. : Mauritius, lofty mountains of the island; Bourbon.

N. (Last.) Thelypteris, Desv, Hab. : Angola.

N. (Last.) Filix-mas, Rich. Hab. : Bourbon, Mauritius, Zambesi Land; Tropical Western Africa; Moramballa, alt. 2,500 feet; Island of St. Thomas, alt. 6,000 feet; Cape de Verdes.

N. (Last.) Welwitschii, Baker. Hab. : Angola. \$.

N. (Last.) spinulosum, Desv. Hab.: Bourbon.

N. (Last.) sparsum, Dor. (N. purpurascens, Hook.). Hab. : Mauritius,

N. (Last.) athamanticum, Hook. Hab.: Angola.

N. (Last.) inæquale, Hook. Hab.: Comoro Isles; Angasilia Island, volcano, between 3,600 and 6,000 feet. $K u h n$.

N. (Last.) hirtum, Hook. Hab. : West Tropical Africa.

N. (Last.) squamisetum, Hook. Hab.: Fernando Po, Clarence 
Nephrodium-continued.

Peak, alt. 4,000 feet; Mount Kilimandjaro, district of Dschagga, 5,500-7,800 feet. Kuhn.

N. (Last.) Napoleonis, Bory. Hab.: St. Helena, close to Napoleon's tomb, on walls and rocks near Diana's Peak, alt. 2,000 feet. $\$$.

N. (Last.) Ascensionis, Hook. Hab.: top of Green Mountain, Ascension Island, alt. 1,200-1,800 feet. J. D. Hooker. $\$$.

N. (Last.) cognatum, Hook. Hab.: Diana's Peak, St. Helena, alt. 2,700 feet. $\$$.

N. (Jast.) Bojeri, Baker (N. Boryanum, Hook.). Hab.: Mauritius and Bourbon. $\$$.

N. (Last.) odoratum, Baker (N. eriocarpum, Dec.). Hab. : Mauritius, Cape de Verdes.

N. (Last.) subquinquefidum, Hook. (N. Vogelii, Hook.). Hab. : Angola, Guinea Coast, Senegambia, Fernando Po: Island of Nossibeh. Houk. Cent. Ferns, t. 21.

N. (Last.) membranifolium, Presl. Hab.: Madagascar.

N. (Last.) oppositum, Hook. Hab. : Bourbon and Mauritius.

N. (Last.) Boryanum, Baker (non Hook.). Hab. . Bourbon.

N. (Last.) catopteron, Hook. Hab.: Mauritius; West Tropical Africa, Island of St. Thomas, Fernando Po, on Clarence Peak, 5,000 feet elevation.

N. (Last.) subglandulosum, Baker. Hab.: Bourbon. \$.

N. (Last.) hispidum, Hook. Hab. : Mauritius.

N. distans, Hook. Hab.: Madagascar, Johanna Island.

N. unitum, R. Br. (non Sieb.; N. propinquum, R. Br.). Hab. : Mauritius, Luabo River, Zambesi; on Mount Kilimandjaro, Dschagga district, 5,500-7,800 feet.-Kuhn. Fernando Po.

N. pallidivenium, Baker (Polyp., Hook.). Hab. : River Bagroo, Guinea Coast, and on the shores of Lake Nyassa. $\$$.

N. cucullatum, Baker (N. unitum, Sieb.). Hab.: Bourbon and Mauritius, Seychelles.

N. venulosum, Hook. Hab.: Fernando Po. \$.

N. arbuscula, Desv. Hab.: Mauritius and Bourbon. Hook. Cent. Ferns, t. 22.

N. pennigerum, Hook. Hab. . Johanna Island; Angola, Guinea Coast.

N. molle, Desv. Hab. : Bourbon and Mauritius ; (var. violascens, Link) Comoro Isles; Island of Angasilia, near the city of Kitanda. - Knlm. Sierra Leone, Guinea Coast.

N. (Sagenia) subtripbyllum, Baker. Hab.: Mauritius, Johanna Island.

N. (Sagen.) latifolium, Bater. Hab. . Guinea Coast.

N. (Sagen.) Pica, Baker (Aspid., Desv.). Hab. . Mauritius and Bourbon. \$s.

N. (Sagen.) Barteri, Bater. Hab. . Fernando Po. \$.

N. (Sagen.) cicutarium, Baker (Aspid., Sw.). Hab. . Angola, Guinea Coast, Prince's Island. Eastern 'Tropical Africa, Johanna Island; Moramballa, elevation 300 feet; Zambesi Land, Bourbon and Mauritius.

\section{Nephrolepis.}

N. exaltata, Schott. Hab.: Mauritius, Zambesi Land; Angola, Guinea Coast. 
Nephrolepis-continued.

N. acuta, Presl. Hab. : Mascaren Isles, Galega Island, Comoro Isles, Angrasilia, Johanna Island, Seychelles, Madagascar, Mount Kilimandjaro, Zambesi Land; Angola, Guinea Coast, Senegambia.

N. ramosa, Baker (Neph. oblitera, Hook.). Hab.: Tropical West Africa, Sierra Leone ; Angiama, Lower Niger, climbing on small trees, and adhering to them like ivy - caudices ten feet long. Fernando Po, St. Thomas's Island, Angola, Johanna Island.

\section{Oleandra.}

O. neriiformis, Cav. Hab. . Guinea Coast.

O. articulata, Cav. Hab.: Mascaren Isles, Seychelles, Guinea Coast, Angola.

\section{Polypodium.}

P. (Pheg.) obtusilobum, Desv. Hab. : Mauritius and Madagascar ; Camernon Mountains, Fernando Po. $\$ \$$.

P. (Pheg.) Sieberianum, Kaulf. Hab.: Mauritius and Bourbon. \$.

P. (Pheg.) molle, Roxb. (P. Dianeæ, Hook.). Hab. : St. Helena, on Diana's Peak, in dense woods, where it grows 3-5 feet high. \$. P. (Pheg.) rufescens, Blume. (P. Barterianum, Hook.). Hab.. Fernando Po.

P. (Pheg.) Mannianum, Hook. Hab. : Fernando Po. \$.

P. (Pheg.) Bojeri, FIook. (P. sessilifolium, Hoo\%.). Hab. . Mauritius and Bourbon. $\$$.

P. (Pheg.) punctatum, Thumb. (P. rugulosum, Labill.). Hab. : St. Helena, Fernando Po, Bourbon.

P. (Pheg.) Vogelii, Hook. Hab.: Fernando Po. \$.

P. (Pheg.) nigritianum, Baker. Hab. : Fernando Po. \$.

P. (Goniopteris) proliferum, Presl. Hab. : Angola, Zambesi Land.

P. (Goniopt.) arthrothrix, Hook. Hab.: Madagascar. \$.

P. (Goniopt.) oppositifolium, Hook. Hab.: Peak of Island of St. Thomas, W. Africa, alt. 5,000 feet. \$.

P. (Goniopt.) unitum, Hook. Hab.: Cameroon Mountains.

P. (Dictyopteris) Cameroonianum, Hook. Hab.: Cameroon Mountains, alt. 3,000 feet. \$\$

P. (Dicty.) sparsiflorum, Hook. Hab.: Sierra de Crystal and Old Calabar, West Tropical Africa.

P. (Grammitis) marginellum, Swz. Hab.: St. Helena, Diana's Peak, Cape Verdes.

P. multifidum, Bory. Hab.: Bourbon and Mauritius. \$.

P. barbatulum, Baker. Hab.: Bourbon. \$\$.

P. serrulatum, Mett. Hab. : Madagascar, Mauritius, Guinea Const. (Xiphopteris Jamesoni, Hook. Second Cent. Ferns, t. 14.)

P. leucosorum, Bojer. Hab.: Mauritius and Bourbon. Hook. Cent. Ferns, t. 42 and 43 . \$\$.

P. villosissimum, Hook. Hab.: West Coast of Africa, Sugarloaf mountains, Sierra Leone; Island of St. Thomss, alt. 6,000 feet; and Fernando Po. $\$$.

P. trichomanoides, Sivz. Hab.: Ascension Island, summit of Green Mountain, alt. 2,800 feet.

P. parvulum, Bory. Hab. : Bourbon, Mauritius, Zambesi Land. 
Polypodium-continued.

P. cultratum, Willd. (P. elasticum, Bory.). Hab.: Mauritius, Bourbon, Fernando Po.

P. rigescens, Bory. Hab.: Bourbon, Fernando Po. Hook. \& Gr. Ic. Fil. t. 116.

P. argyratum, Bory. Hab.: Bourbon and Mauritius. \$\$.

P. (goniophlebium) incanum, Swz. (P. Eckloni, Kze.). Hab.: Zambesi Land.

P. (Niphobolus) adnascens, Swz. (P. spissum, Bory.). Hab.: Bourbon, Mauritius; Cameroon Mountains.

P. (Niph.) Africanum, Mett. Hab. . Zambesi Land, Angola, Guinea Coast; Ambas Bay, Cameroons.

P. (Phymatodes) lineare, Thunb. (P. loriforme, Wall.). Hab.: Bourbon and Mauritius; Fernando Po, alt. 3,000 feet ; St. Thomas, alt. 5,000 feet; Angola.

P. (Phym.) lanceolatum, Lirn. (P. lepidotum, Willd.). Hab.: Madagascar, Mascaren Isles; St. Helena or IDiana's Peak, Zambesi Land, Moramballa Hill, alt. 3,000-3,500 feet. Sierra Leone; Cameroon Mountains, alt. 9,000 feet.

P. (Phym.) lycopodioides, Linn. Hab.: Angola, Grand Bassa, banks of the Nun, Fernando Po, Sierra Leone; Bourbon and Mauritius, Madagaecar, Nossi-beh Island.

P. (Phym.) Melleri, Baker. Hab. . Madagascar. \$.

P. (Phym.) irioides, Lam. Hab.: Bourbon, Mauritius, Madagascar, Zambesi Land, Angola, Guinea Coast, Angola, Siberia.

P. (Phym.) phymatodes, Linn. Hab. Bourbon, Mauritius, Madagascar, Johanna Island, Angasilia Island, Comoro Isles, Nossibeh, Zambesi Land, Guinea Coast, Fernando Po, Prince's Island.

P. (Drynaria) Willdenovii, Bory. Hab. : Bourbon and Mauritius, Comoro Islands, Fernando Po. \$

P. (Dry.) propinquum, Wall. Hab.: Prince's Island, Fernando Po. (P. Willdenovii, Hook. Gard. Ferns, t. 35, non Bory.)

\section{Nothochlaena.}

N. lanuginosa, Desv. Hab. : Cape de Verde Islands.

N. inæqualis, Kze. Hab.: Angola.

N. Marantæ, R. Br. Hab. . Cape de Verdes. Abyssinia.

\section{Monogramme.}

M. graminea, Schls. (M. linearis, $K(f s$.). Hab.: Bourbon and Mauritius, Seychelles.

M. graminoides, Baker (M. furcata, Desv.). Hab. . St. Helena, Guinea.

\section{Gymnogramme.}

G. (Leptogramme) Totta, Schlecht. (G. Lowei, Hook. \& Gr. Ic. Fil. t. 89). Hab.: Fernando Po, on the Peak, 1,500 feet; and on the Cameroons, 7,500 feet. Abyssinia.

G. cordata, Schlecht. Hab. : St. Helena, Angola.

G. Haughtoni, Hook. MSS. (G. cordata, Hook. in part). Hab. : St. Helena, alt. 2,400 feet. $\$$.

G. Jaranica, Blume. Hab. : Tropical West Africa ; Fernando Po, on the Peak, 10,000 feet; Island of St. Thomas, 4-5,000 feet; Cameroons, 4-7,000 feet. 
Gymnogramme-continued.

G. cheilanthoides, Kaulf. Hab.: Mauritius. Hook. \& Gr. Ic. Fil. t. 24 .

G. leptophylla, Desv. Hab. : Abyesinia.

G. Ascensionis, Hook. Hab.: Ascension Island, on rocks and banks on the Green Mountain, alt. 1,200-1,800 feet. Hook. Second Cent. Ferns, t. 67 . \$.

G. (Ceropteris) calomelanos, Kaulf. Hab.: Tropical West African Islands ; Fernando Po ; Prince's Island.

G. (Selliguea) lanceolata, Hook. Hab.: Guinea Coast, Sierra Leone, Brass ; Fernando Po, alt. 3,000 feet; Cameroon Mountains, common, alt. 4-7,000 feet; Bourbon and Mauritius.

\section{Meniscium.}

M. pauciflorum, Hook. Hab.: Sierra del Crystal, West Tropical Africa. \$\$.

\section{Antrophyum.}

A. immersum, Mett. MSS. (A. pumilum, $K l f s$.). Hab.: Bourbon, Mauritius. Hook. \& Gr. Ic. Fil. t. 46. \$.

A. Mannianum, Hook. Hab. : West Tropical Africa, trunks of trees on the Peak of Fernando Po, alt. 3,000 feet; Cameroon Mountains, alt. 3-4,000 feet, and Bagroo River. Hook. Second Cent. Ferns, t. 73 . $\$$.

A. Boryanum, Kaulf. Hab. : East Coast of Africa, Mauritius and Bourbon, Comoro Isles, Johanna Island, Madagascar. \$.

A. giganteum, Bory. Hab.: Mauritius and Bourbon. \$.

\section{Vittaria.}

V. elongata, Swz. Hab.: Mascaren Isles, Seychelles, Comoro Isles, Johanna Island, Mayotte; Tropical W. Africa, Benin ; Fernando Po, alt. 4,000 feet ; Sierra Leone, Kingdom of Oware, River Niger.

V. (Tæniopsis) lineata, $S w z$. Hab. : Fernando Po, alt. 4,000 feet; Quorra, south of the Line, Kingdom of Oware, Sierra Leone, Cameroon Mountains.

V. (Tæniop.) scolopendrina, Thw. (V. Zeylanica, Fée.). Hab. : Seychelles and Mozambique.

\section{Trenitis.}

T. angustifolia, $R$. Br. Hab. : Mauritius.

Drymoglossum.

D. carnosum, Hook. ( $\beta$ D. subcordatum, Fée.). Hab.: Bourbon.

\section{Acrostichum.}

A. simplex, Swz. ( $\beta$ A. martinicense, Desv.). Hab. : Madagascar. -Kuhn. Sierra Leone (?).

A. conforme, Swz. Hab.: Prince's Island and Brass River, Mauritius, Bourbon, St. Helena.

A. palustre, Hook. Hab.: Tropical West Africa, Guinea Coast, banks of the Onitoha and Sierra Leone. In swamps, Ambas Bay; Cameroon Mountains.

A. Sieberi, Hook. \& Gr. Hab. : Mauritius and Bourbon, Fernando Po. Hook. \& Gr. Ic. Fil. t. 237.

A. hybridum, Bory. Hab.: Bourbon and Mauritius, Comoro Isles, Angasilia Island; volcauic mountains of the Cameroons, alt. 8,000 feet; Peal of Fernando Pn, alt. 4-5,000 feet. 
Acrostichum-continued.

A. Aubertii, Desv. Hab.: Bourbon, Zambesi; on the vegetable mould among the dense jungle of Mount Dzomba, alt. 6,500 feet. Fernando Po, alt. 7,000 feet, on the Peak.

A. dimorphum, Hook. \& Grev. Hab.: St. Helena, on rocks and walls; on the summit of Diana's Peak; peculiar to the summit of the little Island of St. Helena. Hook. \& Gr. Ic. Fil. t. 145; and Hook. Second Cent. Ferns, t. 90. \$\$.

A. viscosum, Sroz. Hab. : Bourbon and Mauritius, Seychelles, Comoro Isles, Johanna Island, Angola ; Fernando Po, alt. 2,000 feet.

A. spathulatum, Bory. (A. piloselloides, Presl.). Hab. : Mascaren Islands ; Madagascar.

A. succisæfolium, Thouars. Hab.: Bourbon and Mauritius; Tristan d'Acunha.

A. cinnamomeum, Baker. Hab.: Cameroon Mountains and Clarence Peak, Fernando Po. \$\$.

A. obductum, Kaulf. Hab.: Bourbon and Mauritius. \$\$.

A. tomentosum, Bory. (A. heterolepis, Fêe.). Hab.: Bourbon and Mauritius, Madagascar. \$\$.

A. squamosum, Swz. Hab. : Bourbon, on trees; interior of Madagascar, Comoro Isles, Johanua Island, Angasilia Island; West Tropical Africa, Sugar-loaf Mountain ; Cameroon Mountains, 6,000 feet.

A. (Stenochlæna) sorbifolium, Linn. Hab. : Bourbon, Mauritius, Madagascar, Comoro Isles, Johanna Island; Fernando Po, Prince's Island, River Nun, River Niger.

A. (Steno.) decrescens, Baker. Hab.: Cameroon Mountains. \$\$.

A. (Steno.) tenuifolium, Baker (A. Meyerianum, Hook.). Hab.: Mauritius, Madagascar, Nossi-beh.

A. (Polybotrya) bifurcatum, Sroz. Hab.: St. Helena, abundant, on wet rocks and mossy banks, to an alt. of 1,000 feet; Island of Ascension. \$. Hook. Second Cent. Ferns, t. 91.

A. (Aconiopteris) subdiaphanum, Hook. \& Grev. Hab.: St. Helena, on trunks of trees, summit of Diana's Peak. $\$ \$$

A. (Gymnopteris) Gaboonense, Hook. Hab.: Gaboon River, West Tropical Africa; Angola, 1-2,400 feet. $\$$.

A. (Gym.) repandum, Blume. Hab.: Seychelles.

A. (Gym.) punctulatum, Linn. Hab.: East Tropical Africa, Bourbon and Mauritius, Comoro Isles, Johanna Island; Nossi-beh, near Madagascar; West Tropical Africa, Fernando Po, Prince's Island, Guinea Coast; Angola. \$.

A. (Gym.) rirens, Wall. Hab.: Angola, Fernando Po, Sierra Leone, Senegal River.

A. (Chrysodium) fluviatile, Hook. Hab. : Guinea Coast, Fernando $\mathrm{Po}$, on the banks of the River Niger. $\$ \$$.

A. (Chrys.) Heudelottii, Hool. Hab. : Sierra Leone, Senegambia, Zambesi Land; Manganja country, alt. 3,000 feet, growing in the rorky river bed. \$ Po. \$.

A. (Ghrys.) salicinum, Hook. Hab.: Sierra Leone, Fernando

A. (Chrys.) aureum, Linn. Hab.: Seychelles; Bourbon, Mauritius, Madagascar, Zambesi Land; Angola, Guinea Coast, Sierra Leone, Fernando Po, Prince's Island, Congo. 
Acrostichum-continued.

A. (Hymennlepis) spicatum, Linn. Hab.: Bourbon, Mauritius, Madagascar.

\section{Platycerium.}

P. alcicurne, Desv. Hab. : Mascaren Isles, Seychelles, Comoro Isles, Johanna Island, Mayotte ; Madagascar, near Mombas.

P. æthiopicum, Hook. Hab. : Guinea Coast, Angola, Senegambia, Sierra Leone, Niger-probably abundant; Fernando Po, Prince's Island; Nun River, frequent on trees. Athiopia. Hook. Gard. Ferns, t. $9 . \$$.

\section{Osmunda.}

O. regalis, Linn. Hab.: Bourbon and Mauritius, Madagascar, Zambesi Land, Angola.

\section{Schizaea.}

S. pectinata, Sm. Hab. : St. Helena.

S. digitata, Swz. Hab. . Madagascar.

S. (Lophidium) dichotoma, Shuz. Hab.: Bourbon, Mauritius, Madagascar.

\section{Anemia.}

A. tomentosa, Swz. Hab.: Angola. Kuhn.

\section{Mohria.}

M. caffrorum, Desv. (M. thurifraga, Sw: ). Hab.: Mauritius, Bourbon, Madagascar.

\section{Ingoodium.}

L. scandens, Sroz. Hab. : Guinea Coast, Senegambia, River Niger, Bagroo River.

L. pinnatifidum, Swr. Hab.: Angola, Guinea Coast, Fernando Po, River Niger, Congo.

L. subalatum, Bojer. Hab. : Madagascar, Comoro Isles, Johanna Island. \$5.

L. (Hydroglossum) lanceolatum, Desv. Hab. : Bourbon, Madagascar, Nossi-beh, Comoro Isles, Mayotte. \$.

\section{Angiopteris.}

A. evecta, Hoffm. Hab. : Madagascar, Bourbon, Prince's Island. Hook. \& Gr. Ic. Fil. t. 36.

\section{Marattia.}

M. fraxinea, Smith. Hab. : Guinea Coast, Angola, Fernando Po, Bourbon, Mauritius, Comoro Isles, Mayotte, Madagascar, Zambesi Land; (M. purpurascens, Le Vriese) Ascension Island.

\section{Ophloglossum.}

O. lusitanicum, Linn. Hab.: Guinea Coast; Angola, district Huilla; St. Helena.

O. rubellum, Welw. MSS. Hab.: Pungo Andongo; Angola, 2,4003,800 feet. $\$$.

O. nudicaule, L. fil. Hab. : Guinea Coast, Angola.

O. vulgatum, L. Hab.: Guiner Coast, Angola, St. Helena, Bourbon, Mauritius; Abyssinia.

O. reticulatum, Linn. Hab. : Guinea Coast, Angola, Zambesi Land, Mascaren Isles.

O. (Ophioderma) pendulum, Linn. Hab.: Madagascar, Bourbon and Mauritius. 
Ophioglossum-continued.

O. (Cheiroglossa) palmatum, $L$. Hab.: Bourbon, very rare; Seychelles. Hook. Ic. Plant. t. 4.

\section{CAPE COLONY AND NATAL, TRISTAN D'ACUNHA.}

\section{Gleichenia.}

G. polypodioides, Sm. Hab.: Cape Colony, probably chiefly on the mountains near the Cape Colony, at an elevation of from 1,5004,500 feet. 'Summit of Table Mountain.'-C. Bunbury. Macalisberg; Transvaal Krauskloof, near Pinetown, Natal, 1-6,000 feet. J. Sanderson.

G. (Mertensia) umbraculifera, Moore. Hab. : Natal, open banks of streams, 1-2,500 feet.-J. Sanderson. Cape of Good Hope, Kumahala. \$.

\section{Cyathea.}

C. Dregei, Kze. Hab. : Natal, Macalisberg, as far as the Zambesi, S. lat. $20^{\circ}$; rocky valley at the great cataract between Omsamwubo and Omsamcaba; Moramballa, alt. 3,000 feet. \$.

\section{Femitelia.}

H. Capensis, R. Br. Hab. : moist watery places, Cape of Good Hope. In mountain ravines in many parts of the Cape Colony, there is a noble forest of this fern in the moist woods above 'Paradise,' on the east side of Table Mountain. 'Zwellendam, by the side of a small stream in a deep wooded ravine among the mountains.'-C. Bunbury. Natal.

\section{woodsia.}

'W. Burgessiana, Gerr. MSS. Hab.: Tugela Rirer, Natal. \$.

\section{Eymenophyllum.}

H. rarum, $R \cdot B r$. Hab. : Natal, and Cape Colony, 'ravines of Table Mountain.'-W. H. Hxrvey. Near Graham's Town.

H. obtusum, Hook. et Ain. Hab. : on Table Mountain, Cape of Good Hope.

H. æruginosum, Carm. Hab. : on rocks, Tristan d'Acunha. \$.

H. Tunbridgense, Sm. (H. Dregeanum, Presl.; H. Wilsoni, Hook.). Hab. : Cape of Good Hope, Natal.

\section{Trichomanes.}

T. pusillum, Swz. (T. quercifolium, Hook. \& Gr. Ic. Fil. t. 115). Hab. : Kranz-kop, Natal.

T. Filicula, Bory. Hab. : Cape Colony, Natal.

T. pyxidiferum, L. Hab. : Cape of Good Hope ; Natal, on moist stones and trees in shady ravines, Field's Hill, near Richmond, \&c. 1-2,500 feet. J. Sanderson; Hook. \&. Gr. Ic. Fil. t. 206.

T. tenerum, Sprengel (T. angustatum, Carm.). Hab.: Island of Tristan d'Acunba. Hook, \& Gr. Ic. Fil. t. 166.

T. rigidum, Siv. (T. strictum, Hook. \& Gr. Ic. Fil. t. 122). 


\section{Trichomanes-continued.}

Hab. : Cape Colony; Natal, under shady banks; the Dell, Aldercliffe, \&c., elevation 800 feet. J. Sanderson.

\section{Davallia.}

D. nitidula, Kunze. Hab. : Natal.

D. Speluncm, Baker (D. polypodioides, Hook.). Hab. : Macalisberg Mountains, west of Natal.

D. concinna, Schrad. (D. Schimperi, Hook.) Hab. : Cape Colony ; Natal, on trees in wooded ravines; Townhill; Maritzburg, elevation 2,500 feet. Exceedingly hygrometric. J. Sanderson.

\section{Cystopteris.}

C. fragilis, Bernh. Hab. : Kaffre Country, Cape of Good Hope.

\section{Indsaya.}

L. ensifolia, Swz. Hab.: Cape of Good Hope, Graham's Town, Natal, under a bank by the stream the Dell, Aldercliffe, rare; elevation 800 feet. J. Sanderson; Hook. Gard. Ferns, t. 62.

\section{BHantum.}

A. caudatum, Linn. (A. rhizophorum, Srw.). Hab.: Cape of Good Hope.

A. Capillus-veneris, $L$. Hab. : Cape Colony, Algoa Bay, Uitenhage, forests of Krakakamma; Natal, in moist shady ravines, 500-2,500 feet.-J. Sanderson. (Var. latissimum, Kze.) Tristan d'Acunba.

A. Athiopicum, Linn. Hab.: South Africa, frequent; Natal, Cape Colony ; 'moist rocks in a deep ravino behind Simon's Town.' C. Bunbury.

\section{Tonchitis.}

L. pubescens, Willd. Hab. : Cape Colony; Natal, shady ravines; Field's Hill, Maritzburg; Townhill, 1-2,500 feet. J. Sanderson.

\section{Eypolepis.}

H. anthriscifolia, Presl. Hab.: Cape Colony; Natal.

H. Bergiana, Hook. Hab.: Cape Colony, Kaffraria, and Natal; Townhill, Maritzburg, wooded ravines, 2,500 feet. J. Sanderson.

\section{Chollanthes.}

Ch. pteroides, Swz. Hab.: Cape of Good Hope, abundant in rocky mountainous places, from 1-3,000 feet of elevation. 'In rocky ravines of Table Mlountain.'-C. Bunbury. Natal.

Ch. Capensis, Swz. (Hypolepis, Hook.). Hab.: Cape Colony, ' in moist crevices of rocks in a deep ravine behind Simon's Town.' C. Bunbury.

Ch. hirta, Swz. Hab.: Cape of Good Hope, Table Mountain ; 'in shady places, near Bushman's River, between Uitenhage and Graham's Town.'-Zeyher. Extending east to Port Natal, thence north to Macalisberg.

Ch. multifida, Swz. Hab.: Cape of Good Hope, in rocky and stony places, extending as far as Albany, Table Mountain.-C. Bunbury. Howison's Poort, near Grabam's Town.-Zeyher. Natal.

Ch, induta, Kunze. Hab.: Cape Colony; rocky shady places in Sneeuwbergen. \$. 


\section{Pellaea.}

P. auriculata, Link. Hab. : rocky mountains about Cape Town, Clanwilliam district; 'Paarl, among rocks in shady places.'- $C$ ' Bunbury. Natal. Hook. \& Gr. Ic. Fil. t. 116.

P. geraniæfolia, Fee. Hab. : Cape Colony.

P. deltoidea, Baker (Cheilanthes, Kze.). Hab. : Cape Colony, near Zilverfontein in Namaqua Land, and Goedmanskral, in fissures of rocks. \$.

P. profusa, J.Sm. (Cheilantbes, Kze.). Hab.: near Zilverfontein in Namaqua Land, and Cape of Good Hope. \$\$.

P. robusta, Hook. Hab. : Stony Mountains in Namaqua Land. \$.

P. andromedæfolia, Fée. Hab. : Cape Colony.

P. Boivini, Hook. Hab. : Macalisberg.

P. consobrina, Hook. Hab.: South Africa, among Mimose; Key River, woods in Uitenhage ; clefts of rocks in the Witbergen Mountains; Graham's Town; Natal.

P. hastata, Link. Hab. : Cape Colony, ascending to 4,000 feet; mountain districts, growing in rocky places; 'Graham's Town, in crevices of rocks.' C. Bunbury.

\section{Pteris.}

P. Burkeana, Baker (Pteris Burkeana, Hook.). Hab. : Natal.

Pt. longifolia, $L$. Hab. : Macalisberg.

Pt. Cretica, $L$. Hab.: Cape Colony; in forest on the Van Stader River; Natal, near Macalisberg.

Pt. quadriaurita, Retz. Hab.: Natal.

Pt. flabellata, Thunb. Hab. : Cape of Good Hope, Table Mountain, Uitenhage, 'Forests of Kralkakamma.'-Zeyher. Macalisberg, Natal.

Pt. aquilina, $\dot{L}$. Hab. : S. Africa, abundant. 'Common on the lower slopes of the mountains about Cape Town.'-C. Bunbury. Macalisberg, Natal.

Pt. pedata, Linn. Hab. : Natal.

Pt. incisa, Thumb. Hab.: Cape Colony, by a stream in the Krauskloof, Natal.-J. Sanderson. Tristan d'Acunha.

\section{Iomaria.}

L. attenuata, Willd. (L. gigantea, Kaulf.). Hab. : Cape of Good Hope; forests of Krakakamma, Uitenhage; Table Mountain, forests of George, Graham's Town, Albany; Natal, Macalisberg.

L. alpina, Spreng. Hab.: Tristan d'Acunha, scarce in Kerguelan's Land, apparently confined to Cumberland Bay.

L. punctulata, Kunze. Hab.: Cape Colony. 'Table Mountain, among rocks and stones in the great ravine.'-C. Bumbury. Graham's Town; Natal, frequent; ravine, Field's Hill, 1,000 feet. $J$. Sanderson.

L. procera, Spreng. (L. Capensis, Willd.). Hab. : Table Mountain, Constantia, Uitenhage; Natal.

L. Boryana, Willd. (L. Magellanica, Desv.). Hab. : Macalisberg, by streams and open banks, 1-2,500 feet.-J. Sanderson. Tristan d'Acunha.

\section{Blechnum.}

B. australe, Linn. Hab.: Cape Colony, 'in the ravines of Table Mountain.'-C. Bunbury. Macalisberg, Natal ; Island of St. Paul, Indian Ocean; Tristan d'Acunha. 


\section{Asplenium.}

A. Kraussii, Moore. Hab.: Boschman's Rand, Natal.

A. Sandersoni, Hook. Hab.: ravine in Field's Hill, Natal. J. Sanderson.

A. Trichomanes, $L$. Hab. : Cape of Good Hope, near Swellendam, Caffraria. Kuhn.

A. monanthemum, $L$. Hab. : Tristan d'Acunha; Cape of Good Hope; Natal.

A. ebeneum, Ait. Hab. : Cape Colony, Graham's Town.

A. erectum, Bory. Hab. : S. Africa, abundant; Graham's Town, in forests on the Van Stader mountrins, near Uitenhage; British Kaffraria, Albany, in the Tsitzikamma; Natal and Macalisberg; Tristan d'Acunha.

A. Prionitis, Kunze. Hab. : Cape of Good Hope, near Graham's Town.-Kuhn. Natal; ravine, Town Hill, Maritzburg, 2,500 feet. J. Sanderson.

A. anisophyllum, Kunze. Hab. : Graham's Town mountains, near the Kat River; British Kaffraria, between the rivers Omsamwubo and Omsamcaba; Natal.

A. obtusatum, Forst. Hab. : Tristan d'Acunha.

A. gemmiferum, Schrad. Hab.: Cape Colony, Natal, Knysma, District George.

A. protensum, Schrad. Hab.: Cape Colony, Natal.

A. Ruta-muraria, Linn. Hab.: Cape Colony.

A. Adiantum-nigrum, Linn. Hab.: Cape Colony ; Natal.

A. solidum, Kunze. Hab. : Cape Colony, Algoa Bay, mountains near Ringter Valley, and near Alice. \$.

A. cuneatum, Lam. Hab. : Cape Colony, Graham's Town, Kaffraria, Natal, Macalisberg.

A. furcatum, Thunb. Hab. : 'Graham's Town, on rocks in rather exposed situations.'-C.Bunbury. Forests of Krakakamma-Zeyher. St. Paul's Rock, Indian Ocean, lat. $38^{\circ}-39^{\circ}$, growing among stones at the edge of a crater (now a lake). Natal.

A. bulbiferum, Forst. Hab.: Natal.

A. varians, Hook. \& Grev. Hab.: Cape Town, Kaffraria, Natal. Hook. \& Gr, Ic. Fil. t. 172.

A. cicutarium, Swz. Hab.: Macalisberg.

A. (Darea) brachypteron, Kunze. Hab.: Natal.

A. (Darea) Dregeanum, Kunze. Hab. : shady valley on a cataract near the Omsamcaba River, Natal. \$.

A. (Darea) flaccidum, Forst. Hab. : Natal.

A. (Darea) rutæfolium, Kunze. Hab. Cape Colony and Natal. 'Graham's Town, on moist shady banks in the deep ravines.'-C. Bunbury. Forests on the Van Stader mountains. Zeyher.

A. (Darea) Thunbergii, Kunze. Hab.: Natal. $\$$.

A. (Athyr.) medium, Hook. Hab.: Tristan d'Acunha, on the table-land. 8 .

A. (Athyr.) Filix-foemina, Bernh. Hab.: Natal.

A. (Athyr.) aspidioides, Schlecht. Hab.: Cape Colony, Natal and Graham's Town. Kuhn.

A. (Hemidictyon) Ceterach, Linn. Hab.: Cape of Gaod Hope. 
Actiniopteris.

A. radiata, Link. Hab. : Macalisberg.

\section{Aspidium.} Natal.

A. (Polyst.) aculeatum, Swz. Hab.: Cape Colony, frequent;

A. (Polyst.) pungens, Kaulf. Hab.: Cape Colony, Natal. \$.

A. (Polyst.) Capense, Willd. (A. coriaceum, Suz.). Hab.: Cape, frequent, and eastward to Uitenhage and Natal. 'Graham's Town, frequent on rocks, often in rather sunny and exposed situations.' C. Bunbury. Krakakamma, a very large state. Tristan d'Acunha.

A. (Polyst.) frondosum, Lowe. Hab.: Natal.

A. (Polyst.) aristatum, Sioz. Hab. : Natal.

A. (Cyrt.) falcatum, Swz. Hab.: forests of British Kaffraria ; Natal.

\section{Nephrodium.}

N. (Last.) patens, Desv. Hab.: Natal, by the Umbilo River, 5-800 feet. J. Sanderson.

N. (Last.) conterminum, Desv. Hab.: Tristan d'Acunha.

N. (Last.) Bergianum, Baker. Hab. . Cape Colony and Natal. $\$$.

N. (Last.) tomentosum, Baker (N.thelypteroides, Hook.). Hab.: Tristan d'Acunba.

N. (Last.) Thelypteris, Desv. Hab.: Cape Colony and Natal.

N. (Last.) Filix-mas, Rich. Hab. : Cape Colony.

N. (Last.) spinulosum, Desv. Hab. : Cape Colony, forests on the Van Stader River. Zeyher.

N. (Last.) athamanticum, Hook. Hab. : Cape Colony, Natal, Macalisberg.

N. (Last.) inæquale, Hook. Hab.: Cape Colony and Natal; wooded ravínes, Maritzburg, 2,500 feet. J. Sanderson. \$.

N. (Last.) catopteron, Hook. Hab. : Cape Colony and Natal.

N. unitum, $R$. Br. (non Sieb. ; N. propinquum, Br.). Hab. : Cape Colony. 'Uitenhage, growing almost in the water, on the murgin of the $Z$ wartkops River.'-C. Bunbury. Natal.

\section{Tephrolepis.}

N. acuta, Presl. Hab. : Natal.

\section{oleandra.}

O. articulata, Cav. Hab. : Natal.

\section{Polypodium.}

P. (Pheg.) aquilinum, Thon ars. Hab.: Tristan d'Acunha, growing in large tufts.

P. punctatum, Thunb. (P. rugulosum, Labill.). Hab. : Tristan d'Acunha.

P. (Goniopteris) proliferum, Presl. Hab. : S. Africa.

P. (Goniopt.) unitum, Hook. Hab.: Natal.

P. (Grammitis) Poppigianum, Mett. Hab. : Cape Colony. \$.

P. vulgare, Linn. Hab. : Cape of Good Hope and Natal.

P. (Goniophlebium) ensiforme, Thunb. Hab.: Cape Colony and Natal.

P. (Gonioph.) incanum, Swz. (P. Eckloni, Kze.). Hab.: Cape Colony ; Natal, on trees, abundant, 800-2,500 feet elevation.-J. Sanderson. Chiefly in the eastern district. Macalisberg, alt. 4-5,000 feet, and Glen Ayres, alt. 1,000 feet. 'Near Graham's Town, grow- 
Polypodium-continued.

ing on trees and shrubs in wooded ravines.'-C. Bunbury. Uitenhage.

P. (Niphobolus) Africanum, Mett. Hab.: Kaffraria, Natal.

P. (Phymatodes) Schraderi, Mett. Hab.: Cape Colony and Natal.

P. (Phym.) lineare, Thunb. (P. loriforme, Wall.). Hab.: Kaffraria, Natal.

P. (Phym.) lanceolatum, Linn. (P. lepidotum, Willd.; Pleopeltis linearis, Klfs.). Hab. : Cape Colony. 'Graham's Town, on old mossy stems of trees in the ravines.'-C. Bunbury. Tongaut, 5001,000 feet elevation.-J. Sanderson. Tristan d'Acunha.

P. (Phym.) lycopodioides, Linn. Hab.: Natal.

P. (Phym.) Mackenii, Buker. Hab.: Natal.

P. (Phym.) normale, Don. Hab.: Kaffraria and Natal.

P. (Phym.) irioides, Lam. Hab. : Natal.

P. (Phym.) phymatodes, Linn. Hab.: Cape Colony and Natal. Bush around Durban, sweet-scented, fronds made by the natives into scent balls. Under 100 feet elevation. J. Sanderson.

\section{rothochlaena.}

N. Rawsoni, Pappe. Hab. : hills between Spehlakel and Komaggas, Namaqua Land. \$o Hook, Second Cent. Ferns, t. 77.

N. inæqualis, Kunze. Hab.: Macalisberg, Natal.

N. Eckloniana, Kunze. Hab. : Cape of Good Hope, near Clanwilliam; Mountain of Uitenhage, Kaffraria; Orange River and Grika Land, Namaqua Land; Macalisberg, on mountain ranges, alt. 3,000 feet. $\$$.

N. Buchanani, Baker. Hab. : Natal. \$.

\section{Monogrammo.}

M. graminea, Schk. (M. linearis, $K l f s$. ). Hab. : Cape Colony.

\section{crmnogramme.}

G. (Leptogramme) Totta, Schlecht. (G. Lowei, Hook. \& Gr. Ic. Fil. t. 89). Hab. : Cape Colony.

G. cordata, Schlecht. Hab. : throughout the Cape Colony, plentiful ; Uitenhage Mountains, Macalisberg, Namaqua Land.

G. cheilanthoides, Kaulf. Hab. : Tristan d'Acunha.

G. leptophylla, Desv. Hab.: Cape of Good Hope, Devil's Mountain.

G. (Cerop.) rosea, Desv. (G. conspersa, Kzв.). Hab. : Natal.

G. (Selliguea) lanceolata, Hook. Hab. : Natal.

vittaria.

V. (Tæniopsis) stricta, Carm. Hab.: Tristan d'Acunha.

V. (Tæniop.) lineata, Swz Hab. : Natal, Caledon, Cape Colony.

\section{Acrostichum.}

A. conforme, Swz. Hab.: Cape of Good Hope, abundant. 'Graham's Town, in mossy crevices of the shady sides of rocks.' $-C$. Bunbury. Tristan d'Acunha.

A. hybridum, Bory. Hab. : Natal.

A. Aubertii, Desv. Hab. : Natal.

A. spathulatum, Bory. (A. piloselloides, Presl.). Hab. : Natal, Tristan d'Acunha.

A. succisæfolium, Thouars. Hab.: Tristan d'Acunha. \$. 
Acrostichum-continued.

A. tenuifolium, Baker (A. Meyerianum, Hook.). Hab. : Natal, Cape of Good Hope.

A. (Chrysodium) aureum, Linn. Hab. . Algoa Bay, Natal.

\section{osmunda.}

O. regalis, Linn. Hab. . Cape Colony, between the rivers Omsamwubo and Omsamcaba. Natal.

\section{Todea.}

T. barbara, Moore (T. africana, Willd.; T. rivularis, Sieb.). Hab. : Cape Colony : 'ravines of Table Mountain, and by water courses among the hills about Graham's Town.'-C. Bunbury. Natal.

\section{Schizaea.}

S. tenella, Kaulf. Hab. . Cape Colony and Natal. \$.

S. pectinata, Sm. Hab.: Cape Colony. 'Summit of Table Mountain; and on a dry sun-burnt rock in the Long Kloof.'-C. Bunbury. On the sands of the Cape Flats. W. H. Harvey. \$.

\section{Anemia.}

A. Dregeana, Kze. Hab.: Cape of Good Hope, between the rivers Omsamwubo and Omsamcaba; Natal. \$.

\section{Mohria.}

M. caffrorum, Desv. (M. thurifragra, Swz.). Hab.: Cape of Good Hope, about Cape Town. Natal.

\section{Marattia.}

M. fraxinea, Smith. Hab. . Cape Colony, between the rivers Omsamcaba and Omsamwubo; Natal.

\section{Ophioglossum.}

O. bulbosum, Michx. Hab.: Tristan d'Acunha.

O. rubellum, Welw. MSS. (var. Dregeanum, Milde). Hab.: Cape of Good Hope.

O. nudicaule, L. fil. Hab. : Cape of Good Hope.

O. vulgatum, L. Hab.: Cape Colony, in the deserts of Karroo and Olifants River. Banks of Bushman River. Zeyher.

O. reticulatum, Linn. Hab.: Cape Colony, Natal, Tristan d'Acunha.

O. (Rhizoglossum) Bergianum, Schlecht. Hab.: Cape Colony, rare. $\$$. 


\title{
V. N. AMERICA.
}

1. SUB-ARCTIC GREENLAND, CANADA, TO ROCKY MOUNTAINS.

2. UNITED STATES, BERMUDA.

3. CALIFORNIA, BRITISH COLUMBIA TO NEW MEXICO.

\author{
$\$$ denotes the only hitherto known localities.
}

1. SUB-ARCTIC GREENLAND, CANADA, AND NEW BRUNSWICK, TO THE NORTHERN BORDERS OF THE U. S., AND WESTERLY TO THE ROCKY MOUNTAINS.

\section{Onoclea.}

O. sensibilis, $L$. Hab. : Canada to the Saskatchawan.

O. Germanica, $W$. Hab. : Canada to the Saskatchawan.

\section{Woodsia.}

W. Ilvensis, $\boldsymbol{R}$. Br. Hab.: Canada, to Hudson's Bay, Bear Lake, and the Rocky Mountains; Montreal, Chatham, Wolfe's Cove, Quebec; Lachute; Rivière du Loup-en-bas, on rocky banks; Sorel. -D. R. Mc Cord. Labrador, Greenland.

W. hyperborea, $R$. $B r$. Hab. : Canada to the Saskatchawan.

W. glabella, $R$. $B r$. Hab.: Canada, Chatham, at the Upper Falls of the Rivière du Loup-en-bas; Great Bear Lake; Disco Island, Greenland; Labrador, British North America. tains.

W. scopulina, Eaton. Hab.: British America, Rocky Moun-

\section{Dlcksonia.}

D. punctiloba, Hook. Hab. : Canada, Quebec, Nova Scotia.

\section{Cystopteris.}

C. fragilis, Bernh. Hab.: Greenland, Labrador, Newfoundland, Canada to Slave Lake, and to the Rocky Mountains; Montreal, Shefford Mountains, near Waterloo, in one spot only; Chatham, very fine; Quebec. McCord.

C. montana, Link. Hab.: shady alpine woods, by streams in the Rocky Mountains. Hook. Flora Boreali Americana.

C. bulbifera, Bernh. Hab. : Canada, Montreal, Chatham; Water100, rare; Quebec, at the Falls of Lorette, north declivity of the river; Upper Falls of the Rivière du Loup-en-bas. McCord. 
Adlantum.

A. pedatum, $L$. Hab.: Canada; Lake Huron, common.

cryptogramme.

C. crispa, R. Br. (C. acrostichoides, $R, B r$.). Hab. : Upper Canada, to Sitka Island and Aretic regions.

\section{Pellaea.}

P. gracilis, Hook. Hab.: Canada, rocks, county of Prescott, on the shore of the River Ottawa; Chatham, Rivière du Loup-en-bas; near Brittania Mills, rare (McCord), to the Saskatchawan.

P. atropurpurea, Link. Hab.: Canada to Bear Lake; British North Anerica, Saskatchawan.

\section{Iomaria.}

L. spicant, Desv. Hab.: NW. America, Sitha Island, Prince William's Sound, Observatory Inlet.

\section{Asplenium.}

A. viride, Huds. Hab.: Greenland, Cape Farewell, Newfoundland, New Brunswick. J. H. Redfield.

A. Trichomanes, L. Hab.: Canada to the Saskatchawan; on rocks at Chatham, in large clumps. D. R. Mc Cord.

A. ebeneum, Ait. Hab.: Canada, rare.

A. angustifolium, Michx. Hab. : Canada, not common; Montreal, larger and smaller mountains, open woods, in company with Nephrodium Goldieanum. McCord.

A. marinum, $L$. Hab.: New Brunswick, Nova Sootia.

A. (Athyr.) thelypteroides, Michx. Hab.: Canada, Montreal, Chatham, Quebec, \&c.

A. (Athyr.) Filix-foemina, Bernh. Hab.: Labrador, Newfoundland, New Brunswick, throughout Canada to the Saskatchawan, and to the alpine woods of the Rocky Mountains, Sitka. Hook. Fl. Bor. Am.

\section{scolopendrium.}

S. (Campt.) rhizophyllum, Hook. Hab.: Canada, and British North America to the Saskatchawan.

\section{Aspidium.}

A. (Polyst.) acrostichoides, Swz. Hab. : from Canada to the Rocky Mountrins,

A. (Polyst.) aculeatum, Swz. Hab.: Canada, Lake Superior. Nephrodium.

N. (Last.) Thelypteris, Desv. Hab. : Canada, common; Montreal, Chatham, Sorel, Quebec, \&c., west to the Saskatchawan.

N. (Last.) Filix-mas, Rich. Hab. : Newfoundland, Canada, along to the Rocky Mountains.

N. (Last.) Goldieanum, Hook. Hab. : Canada, near Montreal.

N. (Last.) marginale, Michx. Hab.: Canada, common, and west to the Saskatchawan.

N. (Last.) cristatum, Michx. Hab.: Canada, Montreal, Chatham, Quebec, and west to the Saskatchawan.

N. (Last.) fragrans, Rich. Hab.: Canada to the Saskatchawan, and high northward to the Arctic Seas.

N. (Last.) spinulosum, Desv. Hab.: Newfoundland, Labrador, Canada, and British North America to the Saskatchawan, and westward to the Pacific. J. H. Redfield. 


\section{Polypodinm.}

P. (Pheg.) Phegopteris, $L$. Hab. : Greenland, Labrador, Newfoundland, Canada; and west to Rocky Mountains, and Russian America.

P. (Pheg.) hexagonopterum, Michx. Hab.: Canada, Waterloo, Chatham, Sorel, Quebec. Mc Cord.

P. (Pheg.) Dryopteris, $L$. Hab.: Greenland, Labrador to the Rocky Mountains, and to Bear Lake; Canada, to Hudson's Bay and Sitka.

P. (Pheg.) alpestre, Hoppe. Hab. : Sitka.

P. vulgare, $L$. Hab. . Canada, to the Saskatchawan and Slave River. Hook. Fl. Bor. Amer.

\section{Osmunda.}

O. Claytoniana, Linn. (O. interrupta, Michx.). Hab.: Canada and Newfoundland.

O. cinnamomea, Linn. Hab.: Newfoundland and Canada.

O. regalis, Linn. (O. spectabilis, Willd.). Hab.: Newfoundland, Labrador, Canada, to the Saskatchawan.

\section{Botrychium.}

B. simplex, Hitch. Hab.: British North America, Canada; between Cumberland House Fort and Hudson's Bay.

B. Lunaria, Swz. Hab. . Greenland, Newfoundland, Canada, to the Saskatchawan and the wet priries of the Rocky Mlountains.

B. ternatum, Swz. (B. lunarioides, Swz.). Hab.: Canada, rather rare; Montreal, Sorel, Quebec; Hudson's Bay territory.

B. virginianum, Swz. Hab. : Newfoundland, Canada from Lake Huron, British North America to Saskatchawan.

\section{UNITED STATES, TO THE ROCKY MOUNTATNS. BERMUDA.}

\section{Onoclea.}

O. sensibilis, $L$. Hab.: meadows and wet places, Florida, to Mississippi and northward. Eaton.

O. germanica, $\boldsymbol{W}$. Hab. . White Mountains, New Hampshire; Pennsylvania.

\section{woodsia.}

W. Ilvensis, $R$. Br. Hab. : exposed rocks, common, especially northward; and southward in the Alleghanies, in crevices of rocks, particularly along the banks of rivers.

W. obtusa, Hook. Hab.: rocky banks and cliffs, North Carolina; Tenessee, and northward.-Eaton. (var. W. Oregana, Eaton.) Crevices of rocks, south shore of Lake Superior, Oregon and Rocky Mountains. $\$$.

W. scopulina, Eaton. Hab. : Rocky Mountains.

\section{Dicksonia.}

D. punctiloba, Hook. Hab.: Portland, Maine, White Mountains, New Hampshire, moist rather shady places; very common, pleasantly odorous. This is the only species of Dicksonia inhabiting temperate climates.-Torrey. In the upper part of North Carolina, 'I'enessee, and northward. Eaton. 


\section{Trichomanes.}

T. Petersii, A. Gray. Hab.: near a waterfall in Hancock County, Alabama, not far from Sipsey River, found only on the face of an isolated sandstone rock, within the reach of the spray of a waterfall; also among mosses sent from Pensacola, Florida. Eaton; Hook. Cent. Ferns, t. $86 . \$$.

T. radicans, \$wz. (T. speciosum, Willd.). Hab. : Hancock County, Alabama, Cumberland Mountaius, Eastern Tenessee. Eaton.

\section{Cystopteris.}

C. fragilis, Bernh. Hab.: Rocky Mountains, shaded cliffs, common and very variable, moist rocks on the mountains of North Carolina, and northward. Eaton.

C. bulbifera, Bernh. Hub.: United States, southward to Virginia, and rocks on the muuntains of North Carolina; shady rocky places, frequent. "The bulblets fall to the ground and form new plants, which are about two years in coming to maturity.' Eaton.

C. montana, Link. Hab. : east side of the Rocky Mountains.

\section{Adlantum.}

A. Capillus-Veneris, $L$. Hab. : Florida, mostly pendent from limestone clifs, Florida, Alabama, and westward. Eaton.
A. Athiopicum, $L$. Hab. : Texas.

A. pedatum, L. Hab.: Virginia, moist woods, shady woods, North Carolina, and northwards. Eaton.

\section{Cheilanthes.}

Ch. vestita, Swz. Hab.: Delaware, Pennsylvania, southward to New Carolina; clefts of rocks, island of New York, and $\mathrm{New}$ Jersey to Illinois and southward, near Augusta, Georgia. Eaton.

Ch. lanuginosa, Nuttall. Hab. Illinois and Wisconsin, Iowa, Missouri, thence westward and southward to the Rocky Mountains. In dense tufts on dry rocks and cliffs.

Ch. gracillana, Eaton. Hab.: Missouri.

Ch. tomentosa, Link. Hab.: North Carolina and Tenessee, French Broad River, North Carolina and Tenessee, and 'southwestward.'-Eaton. Mountains of Virginia (?) and Kentucky.

\section{Cryptogramme.}

C. crispa, $R . B r$. (C. acrostichoides, $R . B r$. ). Hab.: Lake

\section{Pellaea.}

P. gracilis, Hook. Hab. : shaded calcareous rocks, Vermont, and Northern and Central New York to Wisconsin; rare.

P. atropurpurea, Link. Hab. : dry calcareous rocks, but of wide range to the Rocky Mountains, on the mountains of Alabama, and northward mostly on lime-rock.-Eaton. Ozark Mountains west of the Mississippi. J.H. Redfield.

P. Alabamensis, Baker (Cheilanthes Alabamensis, Kunze). Hab. : limestone cliffs on the 'Tenessee and French Broad River, Alabama.-Eaton. Georgia.

\section{Pteris.}

P. densa, Hook. Hab.: Washington.

Pt. longifolia, $L$. Hab. : Key West, Florida. Eaton.

Pt. cretica, $L$. Hab. : shady woods, Middle and East Florida, rocks on the Aprlacha River. 
Pteris-continued.

Pt. aquilina, L. Hab. : Kentucky, New Jersey, New Orleans, St. Louis; Missouri, common everywhere. Var. caudata occurs in South Florida, and along the Gulf coast. Eaton.

\section{Blechnum.}

B. serrulatum, Rich. Hab.: River Aisa-hatcha, Florida; the only known locality on the North American Continent.

woodwardla.

W. Virginica, Smith. Hab. : wet swamps, Vermont, New York; Maine to Virginia and southwards; shallow fords, Florida to Mississippi. Eaton. \$.

W. areolata, Moore (W. angustifolia, Smith). Hab.: bogs and shady banks, Massachussetts, near the coast to Virginia, and southward to Florida; apparently most abundant in the Southern States. \$.

\section{Asplenium.}

A. pinnatifidum, Nutt. Hab. : Pennsylvania, southward to Alabama, rare; cliffs on the Schuylkill and Wissahickon, near Philadelphia, and southward along the Alleghanies; also sparingly westward. Eaton, in Asa Gray Manl.; Hook. Cent. Ferns, t. $27 . \$$.

A. dentatum, $I$. Hab.: Carolina, Florida. Eaton.

A. Trichomanes, $L$. Hab. : United States to Pennsylvania. (A. melanocaulon, Willd.) rocks along the Alleghanies, and northward. Eaton.

A. ebeneum, Ait. Hab. : rocky open woods, rather common throughout the United States to the extreme south; some specimens from New Orleans, 2 feet 4 inches long; Florida, Ozark Mountains west of Mississippi. J. H. Redfield.

A. angustifolium, Mich. Hab. : United States, rich woods; W. New England to Michigan, Kentucky, along the mountains. Asa Gray.

A. ruta-muraria, $L$, Hab. : limestone cliffs, Vermont to Michigan, Virginia, and southward along the mountains; scarce. Eaton, in Asa Gray Manl.

A. montanum, Willd. Hab. : cliffs in the Alleghanies, Pennsylvania to Virginia, mountains of Carolina and Georgia and Alabama. Eaton.

A. rhizophyllum, Kunze. Hab.: shady limestone cliffs, Middle Florida; on the walls of a limestone cave at Scharlock's Spring, Jackson County, Florida. Eaton.

A. (Athyr.) thelypteroides, Michx. Hab.: rich woods, notrare; in the Middle United States and in the upper parts of Georgia. Eaton.

A. (Athyr.) Filix-foemina, Bernh. Hab. : moist woods, common, and very variable throughout the United States; low shady woods in Florida to the Mississippi, and northward. Eaton.

\section{Scolopendrium.}

S. vulgare, Sm. Hab.: shaded ravines, and under limestone cliffs, Chittenango Falls, and near Jamesville, \&c., Onondaga Co., New York.

S. (Campt.) thizophyllum, Hook. Hab.: shaded calcareous 
Scolopendrium-continued.

rocks, W. New England to Wisconsin, and southward, rare or local. - Asa Gray. New York, Vermont, Schuylkill, Kentucky, Pennsylrania. 'A remarkable plant, which by means of copious plantlets, produced from the long, slender, proliferous apices of the fronds, traverses a good deal of ground, and is known in the MSS. by the name of the "Walking Leaf." "Shaded rocks on the mountains of Georgia. Eaton.

\section{Aspidium.}

A. (Polyst.) acrostichoides, Swz. Hab. : the United States, and along the Alleghanies, and from Florida, in shady and rocky woods, westward to the Mississippi. Eaton.

A. (Polyst.) Lonchitis, Swz. Hab. : Lake Superior, Michigan, east side of the Rocky Mountains.

A. (Polyst.) aculeatum, Swz. Hab.: United States, apparently rare; mountains of New Hampshire, Vermont; probably nowhere south of New York.

\section{Nephrodium.}

N. (Lastrea) patens, Desv. Hab.: Florida, low shady woods, to S. Carolina, westward; New Orleans, Texas.

N. (Last.) Noveboracense, Desv. Hab.: swamps and thickets ; common in the United States, low grounds; N. Carolina, and northward. Eaton.

N. (Last.) Thelypteris, Desv. Hab.: United States, swamps and bogs; Florida, and northward Eaton.

N. (Last.) Filix-mas, Rich. Hab. : rocky woods, Keweenaw Peninsula, Lake Superior, and westward.

N. (Last.) Goldieanum, Hook. Hab. : rich and moist woods, from Connecticut to Kentucky, and northwards. Hook. \& Gr. Ic. Fil. t. 102.

N. (Last.) marginale, Michx. Hab.: United States, rocky hillsides in rich roods; common, especially northward. Eaton.

N. (Last.) Floridanum, Hook. Hab.: wet woods, Louisiana and Florida. Hook. Fi. Exot. t. 99 . \$.

N. (Last.) cristatum, Michx. Hab.: United States, swamps; common.

N. (Last.) fragrans, Rich. Hab. : shaded trap-rocks, Falls of the St. Croix, Wisconsin, Lake Superior.

N. (Last.) spinulosum, Desv. Hab. : shady woods in the upper districts of North Carolina, Tenessee and northward.-Eaton. (Var. dilatatum, Gray or Swz.) Summits of the Black Mountains, North Carolina. Eaton.

\section{Tephrolepis.}

N. exaltata, Schott. Hab.: South Florida, Eaton.

\section{Poly podium.}

P. (Pheg.) Phegopteris, L. (P. polypodioides, Fée.). Hab.: United States; damp woods, common northward.

P. (Pheg.) hexagonopterum, Michx. Hab. : shady woods, Florida to Mississippi, westward and northward. Eaton.

P. (Pheg.) Dryopteris, $L$. Hab. : Northern United States, rocky woods. 
Polypodiun-continued.

P. taxifolium, L. (P. Plumula, Willd.). Hab.: Tampa Bay, Florida. Eaton.

P. vulgare, Linn. Hab.: United States, mossy rocks and in shady woods in the upper districts of Alabama, and northward. Eaton.

P. (Gonioph.) incanum, Swo. Hab. : rocks and trunks of trees, Virginia and Ohio to Illinois, southward to Florida and Mississippi.

P. (Phlebodium) aureum, $L$. Hab, : South Florida.

P. (Campyloneuron) Phyllitidis, L. Hab.: South Florida.

\section{vittaria.}

V. (Tæniopsis) lineata, Swz. Hab.: South Carolina, on trees; Florida.

\section{Acrostichum.}

A. (Chrysodium) aureum, Linn. Hab. : coast of Sonth Florida. -Eaton. Bermudas.

\section{Osmunda.}

O. Claytoniana, Linn. (O. interrupta, Michx.) Hab.: United States, low grounds in the upper districts, and northward. Eaton.

O. cinnamomea, Linn. Hab.: low grounds, Florida, and northwards in United States, swamps and low copses, everywhere. Eaton.

O. regalis, Linn. (O. spectabilis, Willd.). Hab. : swamps and wet woods, common in the United States, Florida to Mississippi, and northward. Eaton.

\section{schlzea.}

S. pusilla, Pursh. Hab. : pine barrens of New Jersey. \$.

\section{Anemla.}

A. adiantifolia, Swz. Hab.: Florida, Key West.

\section{Iygodium.}

L. palmatum, Swa. Hab. : Massachussetts to Florida, low shady woods ; not common.

\section{Ophloglossum.}

O. bulbosum, Michx. Hab.: low grounds, Louisiana and Florida.

O. nudicaule, L. fil. Hab. : low sandy places, or occasionally in dry soil, Florida and Georgia. Eaton.

O. vulgatum, Linn. Hab. : temperate United States; in sphagnous meadows and pastures, Tenessee, and northward. Eaton.

\section{Botrychium.}

B. simplex, Hitch. Hab.: United States; Maine to New York, and northward, rare.

B. rutaceum, Swz. (B. lanceolatum, Angst.). Hab.: Northern United States, New Hampshire, \&c.

B. ternatum, Swz. (B. lunarioides, Swz.). Hab. : low shady woods and pastures, rarely in open pine barrens ; Florida, and northwards. Eaton.

B. virginianum, Sicz. Hab. : shady woods, Florida, and northward; common through the United States. 


\section{CALIFORNIA, BRITISH COLUMBIA, COVERING THE SLOPE FROM THE ROCKY MOUNTAINS TO THE PACIFIC AND NEW MEXICO.}

\section{woodsia.}

W. obtusa, Hook. (W. oregana, Eaton, $\beta$ Lyallii, W. Perrianana, Hook. \& Gr. Ic. Fil. t. 68). Hab. : near the sources of the Columbia River, Oregon and Rocky Mountains.

W. scopulina, Eaton. Hab. : Rocky Mountains.

\section{Cystopteris.}

C. fragilis, Bernh. Hab. : British Columbia to Sitka.

\section{Adiantum.}

A. Capillus-veneris, $L$. Hab.: Sierra Madre, New Mexico.

A. Athiopicum, L. Hab. : California, Sierra Madre, Rio Grande. J. $H$. Redfiell.

A. pedatum, $L$. Hab. . California, Columbia River, north-west coast. Hook. Flora Boreali Americana.

\section{Eypolepis.}

H. Californica, Hook. Hab. : California. \$.

\section{Chellanthes.}

Ch. (Adiantopsis) Seemanni, Hook. Hab. : Sierra Madre, New Mexico.

Ch. microphylla, Swz. Hab. : Sierra Madre, New Mexico. $\boldsymbol{J} . \boldsymbol{H}$. Redfield.

Ch. viscosa, Kaulf. Hab.: New Mexico, Sierra Madre.

Ch. Wrightii, Hook. Hab. : from Westem Texas to EI Paso and New Mexico. \$.

Ch. lanuginosa, Nuttall. Hab.: California and New Mexico.

Ch. gracillima, Eaton. Hab.: British Columbia, Oregon, California, New Mexico.

Ch. Fendleri, Hook. Hab.: Rocky Mountains, southward to California, and New Mexico. \$.

Ch. Lindheimeri, Hook. Hab. : between Western Texas and El Paso, New Mexico. $\$$.

Ch. Fatoni, Baker (Ch. tomentosa, Hook.). Hab.: between Western Texas and El Paso, New Mexico. $\$$.

Ch. tomentosa, Link. Hab. : sources of Rio Grande, New Mexico.

\section{Cryptogramme.}

C. crispa, R. Br. (C. acrostichoides, R. Br.). Hab.: Sitka, and the arctic regions southward to Oregon and California; on stony places in the Rocky Mountains, but rare; to the sources of the Columbia, and thence to the grand rapids of the Columbia and the mountains of MacGillivray's River. Hook. Flora Boreali Americana.

\section{Pellea.}

P. Breweri, Eaton. Hab.: California. \$.

P. mucronata, Eaton. Hab. : California, New Mexico, and valley of the Rio Grande. $\$$.

P. aspera, Baker. Hab. : New Mexico. \$.

P. ornithopus, Hook. Hab. : California. \$. 
Pellaa-continued.

P. densa, Hook. Hab.: California, Oregon.

P. andromedæefolia, Fée. Hab.: California, San Francisco, Rio Grande, New Mexico. J. H. Redfield.

P. pulchella, Fée. Hab. : between Western Texas and El Paso, New Mexico.

P. Bridgesii, Hook. Hab. : mountains in the interior of California, Sierra Nevada. \$s.

P. cordata, J. Sinith (P. flexuosa, Link). Hab. : New Mexico.

\section{Pteris.}

Pt. aquilina, L. (Var. lanuginosa, Bory.). Hab.: California, from Cape Mendocino to Puget Sound, and in the open wnods and undulating grounds in the interior of Columbia. Hook. Flora Boreali Americana.

\section{Iomarta.} Fuca.

L. spicant, Desv. Hab. : Nootka Sound and Straits of Juan de

\section{Woodwardia.}

W. radicans, Smith. Hab.: California, Sierra Madre; not known elsewhere in the Now World.

\section{Asplenium.}

A. viride, Huds. Hab. : moist shady rocks on the Rocky Mountains, Russian territory, Chatham Sound.

A. Trichomanes, $L$. Hab.: damp rocks of the Columbia and Puget's Sound; New Mexico.

A. septentrionale, L. Hab. : Rocky Mountains in New Mexico.

A. (Athyr.) Filix-formina, Bernh. Hab.: British Columbia, Oregon; common on the north-west coast, by the shady banks of streams.

\section{Aspidium.}

A. (Polyst.) munitum, Kaulf. Hab.: Western N. America; from Nootka to California, from Monterey in the south, through Oregon territory to Nootlia. The most common of all Ferns, between the parallels of $40^{\circ}$ and $49^{\circ} \mathrm{N}$. lat.; in moist ground of the dark shady pine forests. The roots are roasted on the embers, and constitute an article of food. The fronds are used as garlands by the Indians. Hook. Flo. Bor. Am.

A. (Polyst.) Lonchitis, Swz. Hab.: British North America; apparently rare; British Columbia, at the Cascade Mountains, $49^{\circ}$ N. lat., at 5-6,000 feet elevation above the sea.

A. (Polyst.) aculeatum, Swz. Hab.: near the sources of the Columbia, Sitka, Nootka.

A. (Polyst.) Californicum, Eaton. Hab.: California. \$8.

A. (Cyrt.) juglandifolium, Kze. Hab. New Mexico, Rio Grande. J. H. Redfeld.

\section{Irephrodium.}

N. (Last.) Filix-mas, Rich. Hab. : woods; Monterey in Califormia.

N. (Last.) rigidum, Desv. Hab. : California, New Mexico.

\section{Polypodium.}

P. (Pheg.) Phegopterie, L. Hab. : near the sources of the Columbia River. 
Polypodium-continued.

P. (Pheg.) Dryopteris, $L$. Hab.: British Columbia, on the Pacific; high mountains of the rapids of the Columbia River. Sitka. P. (Pheg.) alpestre, Hoppe. Hab. . Sitka, California, and Oregon.

P. Madrense, J. Sm. Hab. : Sierra Madre, NW. Mexico. \$.

P. vulgare, $L$. Hab. : British and North-west America, on rocks and decayed wood; common; near the confluence of the Columbia with the sea, north to Sitka, rare in California.

P. (Gomiophlebium) Californicum, Kaulf. Hab.: California; moist rocks, Columbia River. \$.

P. (Gonioph.) Scouleri, Hook. \& Grev. Hab.: Straits of Juan de Fuca, Vancouver's Island, Oregon, British Columbia. Hook. \& Gr. Ic. Fil. t. 56. $\$$

\section{Tothochlæena.}

N. sinuata, Kaulf. Hab.: Sierra Madre, New Mexico, Western Texas, and el Paso.

N. ferruginea, Hook. Hab.: New Mexico.

N. (Cincinalis) sulphurea, J. Sm. (N. candida, Hook.). Hab.: New Mexico, California. $\$$.

N. (Cinc.) dealbata, Kunze. Hab. : banks of the Missouri and Arkansas. \$\$.

N. (Cinc.) Fendleri, Kunze. Hab. : New Mexico. \$\$.

\section{Cymnogramme.}

G. pedata, Kaulf. Hab. : New Mexico. J. H. Redfeld.

G. (Ceropteris) triangularis, Kaulf. Hab.: Vancouver's Island and Oregon; California, near San Francisco. Hook. \& Gr. Ic. Fil. t. 153.

G. (Cerop.) tartarea, Desv. Hab. : New Mexico. J. H. Redfield.

G. (Selliguea) podophylla, Hook. Hab. : New Mexico; Rio Grande.

\section{Acrostichum.}

A. conforme, Swz. Hab.: Sierra Madre, New Mexico.

\section{Anemia.}

A. mexicana, Klotzsch. Hab.: New Mexico and Texas. Hook. Cent. Ferns, t. 88.

\section{Botrychiam.} Madre.

B. ternatum, Swz. (B. lunarioides, Swz.). Hab.: Nootka, Sierra 


\section{SOUTH AMERICA.}

1. MEXICO, PANAMA, WEST INDIAN ISLANDS.

2. VENEZUELA, N. GRANADA, EQUADOR, PERA, BOLIVIA, GALAPAGOS.

3. GUIANA, BRAZIL, PARAGUAY, URAGUAY.

4. CHILI, LA PLATA, PATAGONIA, FALKLAND ISLES, ETC.

\section{$\$$ denotes the only hitherto known localities.}

\section{MEXICO, TO PANAMA, AND WEST INDIAN ISLANDS.}

\section{Glelchenia.}

G. longissima, Bl. (G. Bancroftii, Hook.). Hab. : Mexico, Guatemala. W. Indies: Jamaica, rare, near the summit of the Blue Mountains.-Grisebach. Martinique, Guadaloupe.

G. revoluta, $H, B, R$. Hab. : summit of the Blue Mountains, Jamaica.

G. pubescens, $H . B . K$. Mertensia immersa, Kaulf.; Hook. \& Gr. Yc. Fil. t. 15 (M. farinosa, Kaulf.). Hab.: Mexico, Guatemala. W. Indies: Cuba; Jamaica, on the summit of the Blue Mountains; Martinique, St. Vincent, Dominica, St. Domingo, Guadaloupe, Porto Rico, Trinidad.

G. pectinata, Presl. (G. glaucescens, Willd.). Hab.: Mexico, Guatemala, Panama. W. Indies: Jamaica, St. Ann's, Blue Mountains, St. Vincent, Martinique, Guadaloupe, Trinidad, Dominica.

G. dichotoma, Willd. Hab. : Panama. W. Indies : Cuba, Jamaica, Martinique, Guadaloupe, Trinidad, Tobago.

\section{Cyathea.}

C. arborea, Sm. Hab. : Jamaica, St. Domingo, Martinique, St. Vincent, and probably the W. Indian Isles generally.

C. Serra, Trilld. Hab.: Mexico, Guatemala (?); Jamaica, in woods above Bath; in mountain woods, St. Mary's; Dominica, St. Vincent, Porto Rico, Cuba, Trinidad, Guadaloupe.

C. insignis, Eat. Hab. : Jamaica, on St. Catherine's Peak, elevation 5,000 feet; Cuba, mountain forests of Vera Cruz. $\$$.

C. Imrayana, Hook. Hab. : Couliaban Mountains, Dominica; a high mountain species, gathered on the Blue Mountain Peak in Jamaica, alt. 5,000 feet; St. Vincent, Guadaloupe.

C. balanocarpa, Eat. Hab. : Cuba. \$. 
Cyathea-continued.

C. gracilis, Griesb. Hab.: Jamaica, Fox Gap.

C. Schanschin, Mett. Hab.: Mexico, Zacualtipan, Guatemala.

C. Mexicava, Schlecht (C. denudans, Kze.). Hab. : river sides, forests of Xalapa, Mexico. $\$$.

\section{Femitelia.}

H. subincisa, Kze. Hab. . Guatemala, forest of Vera Paz; Trinidad.

H. grandifolia, Spr. (H. obtusa, Klfs.). Hab.: W. Indies: Jamaica, Trinidad, St. Vincent, Martinique, St. Kitts.

H. horrida, $B r$. Hab.: W. Indies; St. Domingo, Jamaica, Trinidad, Martinique, St. Vincent, Cuba, Guadaloupe, Dominica.

H. petiolata, Hook. Hab. : Isthmus of Panama, Isle of Gorgona, Mexico.

H. apiculata, Hook. Hab. : Mexico. $\$$.

H. calolepis, Hook. Hab. : Cuba. \$. feet.

II. Wilsoni, Hook. Hab. : Mansfield, near Bath; Jamaica, 1,000

\section{Alsophila.}

A. blechnoides, Hook. Hab. : Guatemala; Isle of Gorgona, west const of Panama. Trinidad.

A. Schiedeana, $P_{r}$. Hab. : Mexico.

A. armata, Pr. Hab. : Island of Gorgona; and Cocos Island, Central America ; Jamaica, in mountain woods ; St. George's. Grisebach.

A. aspera, $B r$. Hab.: W. Indies: Cuba ; Jamaica, in mountain woods, at 3,000 feet alt.; St. Vincent, Martinique, Montserrat, Guadaloupe, Dominica, Porto Rico, St. Kitt's. Hook. \& Gr. Ic. Fil. t. 213-215.

A. ferox, Pr. Hab.: Trinidad.

A. infesta, Kze. Hab.: W. Indies: Dominica, Jamaica, Martinique, Guadaloupe.

A. atrovirens, $P r$. (A. compta, Mart.). Hab.: Tabasco, Mexico.

A. elongata, Hook. Hab.: Central America.

A. Salvinii, Hook. Hab. : Chilissco, Guatemala. \$.

A. Godmani, Hook. Hab.: Coban, Guatemala. \$.

A. pruinata, Klfs. Hab.: Mexico; Jamaica, in mountain woods. Grasebach.

alt. $\$$.

A. Mexicana, Mart. IIab. . Mexico, on mountains 2,400 feet

Hypoderris.

H. Brownii, J. Sm. Hab.: St. Ann's Valley, Trinidad. Hook. Gard. Ferns, t. 24. \$.

\section{Woodsia.}

WV. mollis, J. Sn. Hab. : Mexico, Guatemala. \$.

W. Guatemalensis, Hook. Hab.: Mexico, Guatemala. \$.

\section{Dicksonia.}

D. (C'ibotium) Schiedei, Schlecht \&. Cham. Hab.: Hacienda de la Laguna, Nexico; Xalapa, Guatemala.

D. Sellowiana, Hook. Hab.: Guatemala.

D. coniifolia, Hook. Hab. : Jamaica, Guadaloupe, Nicaragua. 
Dicksonia-continued.

D. Plumieri, Hook. (Davallia "adiantoides, Swz.). Hab: St.

Domingo, Jamaica, Dominica, Trinidad, Guadaloupe, Cuba.

D. adiantoides, $H . B . K$. Hab.: W. Indies: St. Domingo; Jamaica, in woods; Cuba; Mexico.

D. cicutaria, Swz. Hab. : Vera Cruz and Xalapa, Mexico, Guatemala, Cocos Island, Guayaquil. West Indies : Jamaica, in the mountains.

D. apiifolia, Swz. Hab.: Jamaica, Cuba.

D. rubiginosa, Kaulf. Hab. : Vera Cruz, Mexico, Guatemala, Guayaquil. West Indies: Jamaica, Porto Rico.

\section{Eymenophyllum.}

H. asplenioides, Swuz. Hab. : trunks of trees, in "high mountains, Jamaica, Cuba ; Mexico. Hook. Cent. Ferns, t. 56.

H. abruptum, Hook. Hab. : Jamaica, Cuba; Guatemala.

H. axillare, Suv. Hab.: Jamaica, on trunks of trees in the highest mountains; St. Vincent, St. Domingo. Grisebach; Hook. $\&$ Gr, Ic. Fï. t. 124.

H. undulatum, Swz. Hab.: Mexico; Jamaica, in high mountains in ravines; Cuba. Grisebach; Hook. Cent. Ferns, t. 64.

H. myriocarpum, Hook. Hab. : Mexico.

H. polyanthos, Swz. Hab, : Mexico. West Indies, apparently general; Guadaloupe, Cuba; Jamaica, on trees and rocks in the mountains; St. Vincent, Dominica. Grisebach; Hook. \& Gr. t. 128.

H. hirsutum, Swz. Hab.: Jamaica, on trees in high mountains, Trinidad. Hook. \& Gr. Ic. Fil. t. 84.

H. ciliatum, Swoz. (H. Plumieri, Hook. \& Gr. Ic. Fil. t. 123). Hab. : Mexico. West Indies: Jamaica, on trees in the mountains; Dominica, St. Vincent, Trinidad, Cuba. Grisebach.

H. hirtellum, Swz. Hab. : Mexico ; Jamaica, wet banks ; Fox's Pass, St. George's; Guadaloupe, St. Vincent.

H. valvatum, Hook. \& Gr. Hab.: Nicaragua; Guadaloupe, Martinique. Hook. \& Gr. Ic. Fil. t. 219.

H. interuptum, Kze. Hab.: Guadaloupe.

H. sericeum, Swz. Hab. : Mexico ; Cuba ; Jamaica, on trees in high mountains; St. George's; Trinidad. Grisebach.

H. lineare, Swz. Hab. : West Indies: Jamaica, on moist banks, rare; Guadaloupe and Martinique. Grisebach.

H. elegantulum, $I . D . B$. Hab.: Jamaica. Grisebach.

H. Catherinæ, Hook. MSS. Hab.: St. Catherine's Peak, Jamaica, at an elevation of 5,000 feet; Guadaloupe.

H. Tunbridgense, Snith. Hab.: Guatemala, Jamaica. F

H. fucoides, Swz. (H. spinulosum, H. B. K.). Hab. : Mexico; Jamaica, on trees in high mountains; Martinique. Hook. Cent. Ferns, t. 63.

\section{Trichomanes.}

T. elegans, Rudge. Hab.: Gorgona, coast of Panama, and Pacific side of Central America, in shady woods. Guatemala; Trinidad, in marly ravines near Mount Tamana, Irais. Grisebach; Hook. Gard. Ferns, t. 2.

T. botryoides, Kaulf. (T. nanum, Bory.). Hab. : Panama.'

T. spicatum, Hedw. Hab. : Bay of Choco, Isthmus of Panama. $\mathrm{K} 2$ 


\section{Trichomanes-continued.}

West Indies, frequent; woods of Portland, Jamaica ; St. Vincent; Trinidad, in rocky ravines, at the foot of Mount Tocuche.-Girisebach. Guadaloupe. In very wet shaded places, often found incrusted with epiphytal Jungermannix. Hnok. Gard. Ferns, t. 60.

T. lineolatum, Hook. Hab. : Culsa and Jimaica.

T. membranaceum, $L$. Hab. : Tropical America, both the islands and continent, abundant on the trunks of trees. Jamaica, on moist rocks ; St. Kitts, Dominica, St. Vincent; Trinidad, Cuba to French Islands. Grisebach.

T. Godmani, Hook. MSS. Hab.: Guatemala ; Panama.

T. punctatum, Poiret. Hab. : Gorgona, Pacific side of Central America; Martinique, Guadaloupe, Jamaica; Trinidad, growing often intermingled with T. muscoides. Hook. \& Grev. Ic. Fil. t. 236.

T. reptans, Swz. Hab. : Jamaica, on trees; St. Vincent, Cuba, Barbadnes. Hook. \& Gr. Ic. Fil. t. 32.

T. apodum, Hook. \& Gr. Hab.: Tropical America, both islands and mainland; Jamaica, Barbadoes, Cuba; Mexico. Hook.\& Gr. Ic. Fil. t. 117.

T. muscoides, Swaz. Hab. : Jamaica, on trees ; St. Domingo, Dominica, St. Vincent.

T. pusillum, Swz. Hab.: Mexico. West Indies: Jamaica; Trinidad, on trees near the summit of Mount Tocuche, often growing intermingled with T. muscoides.-Grisebach. (T. quercifolium.) Hook. \& Gr. Ir. Fil. t. 115.

T. Krausii, Hook. \& Gr. Hab. : Mexico. West Indies: Dominica, St. Vincent, Trinidad, Berbice, Porto Rico, Guadaloupe; Jamaica, on trees. Hook. \& Gr. Ic. Fil. t. 149.

T. sinuosum, Rich. (T. incisum, Kaulf.). Hab.: MLexico ; Guadaloupe and other West Indian Islands, frequent; Jamaica, on treeferns; St. Vincent, Trinidad, Cuba. Hook. \& Gr. Ic. Fil. t. 13.

T. pinnatifidum, $V . D . B$. Hab. : Jamaica.

T. Bancroftii, Hook. \& Grev. Hab.: Jamaica, common in woods; Portland, St. Vincent. Grisebach; Hook. \& Gr. Ic. Fil. t. 204.

T. macilentum, $V . D . B$. Hab.: Trinidad.

T. superbum, V. D. B. Hab. : Trinidad.

T. Kaulfussii, Hook. \& Gr. (T. lucens, Hook. \& Gr. Ic. Fil. t. 10). Hab.: West Indies: Jamaica, St. Vincent, Dominica, St. Thomas, St. Kitts, Trinidad, Guadaloupe.

T. alatum, Swz. Hab.: St. Domingo, in high mountains of Jamaica; St. Vincent, Trinidad, Guadaloupe and Martinique, in moist mountain woods; Dominica. Hook. \& Gr. Ic. Fil. t. 11.

'T. brachypus, Tzuze. (T. Ankersii, Purker; Hook. \& Gr. Ic. Fit. t. 201). Hab.: West Indies, on trunks of trees; Trinidad, St. Vincent, Berbice, Guadaloupe.

T. pyxidiferum, $L$. Hab. : Mexico. West Indies: St. Vincent, Jamaica, in mountain woods; Guadaloupe. Grisebach.

T. radicans, Sicz. (T. speciosum, Willd.; Hymenoptyllum rupestre, Raddi). Hab.: Mexico, Vera Cruz; Talapa, Tabasco; Jamaica, on trees, and in woods above Port Stewart, Martinique.

T. crispum, $L$. Hab.: Mexico ; Jamaica, on decayed trees ; Martinique, Guadaloupe, St. Vincent, Montserrat, Cuba, Trinidad, heights of Las Cuevas. Grisebach. 
Trichomanes-continued.

T. crinitum, Swz. Hab.: Jamaica, on trees in the mountains; St. Vincent. Grisebach.

T. lucens, Swz. Hab. : Guatemala; Jamaica, on shady soil.

T. pinnatum, Swz. (T. floribundum, H. B. K.). Hab. : Mexico, Jamaica, Barbadoes, Dominica, Trinidad. Hook. Gard. Ferns, t. 8.

T. tenerum, Sprengel. (T, angustatum, Carm.; Hook. \& Gr. Ic. Fil. t. 166). Hab. : Mexico.

T. trichoideum, Swz. Hab.: Mexico, Vera Cruz, Xalapa; Jamaica, on fern stems, in moist woods at 5,000 feet alt. Grisebach; Hook. \& Gr. Ic. Fil. t. 199.

T. scandens, Linn. Hab.: Mexico, Cuba, and Jamaica in the Blue Mountains.

T. rigidum, Swuz. Hab. : Mexico; Jamaica, on tree-rnots; Martinique, Dominica, Barbadoes, and probably general in the W. Indian Islauds.

T. Leprieurii, Kunze. Hab.: Isle of Gorgona, Pacific side of Central America. West Indies: Dominica; Trinidad, in woods. Flook. Gard. Ferns, t. 11.

\section{Davallia.}

1). Imrayana, Hook. Hab.: Dominica, Guadaloupe.

D. Saccoloma, Spreng. Hab.: Isthmus of Panana, Guatemala; Jamaica, interior of Westmoreland Co., and Fox's Gap, St. George; Trinidad, Tocuche.

D. inæqualis, Kunze. Hab.: Guadaloupe; Jamaica, in woods.

D. Speluncæ, Baker (D. Jamaicensis, Huok.). Hab.: West Indies: Jamaica, in moist woods; Cuba.

D. clavata, Swz. Hab.: West Indies: Cuba; Jamaica, on wet rocks; Martinique, Bahamas.

D. uncinella, Kunze. Hab. : Cuba and Porto Rico.

D. aculeata, Suz. Hab.: West Indies: St. Domingo; Jamaica, in dry savannahs; Dominica. Cuba.

D. fumarioides, Swz. Hab.: West Indies : Jamaica, on dry hills;

I). Schlechtendahlii, Presl. Hab. : Mexico and Guatemala, slate district east of Oaxaca, 3-5,000 feet elevation.

\section{Cystopteris.}

C. fragilis, Bernh. Hab. . Peak of Orizaba, Mexico; Guatemala ; Jamaica. Grisebach.

\section{Iindsaya.}

L. Seemanni, J. Sm. ITab. ; Bay of Choco, Panama.

L. trapeziformis, Dry. (L. quadrungularis, Raddi). Hab.: Guatemala. West Indies: Dominica, Cuba, Jamaica, Trinidad, Guadaloupe; Panama.

L. Guianensis, Dry. Hab.: Jamaica, in the Bluefield Mountains; Trinidad.

L. stricta, Dry. Hab. : Mexico, Isle of Gorgona, west of Panama. West Indies: Trinidad; Jamaice, in high mountains. Grisebach. t. 87 .

L. sagittata, Lry. Hab.: Guadaloupe. Hook. \& Gr. Ic. Fil.

\section{Dictyoxiphium.}

D. Panamense, Hook. Hab. : Isthmus of Panama, Pacific coast Guatemala. 
Adiantum.

A. Kaulfussii, Funze, Hab.: Mexico. West Indies: Jamaica, St. Vincent, Cuba, Guadaloupe, Nartinique.

A. obliquum, Willd. Hab.: West Indies: Porto Rico, Guadaloupe, Jamaica, Trinidad, Cuba.

A. Galeottianum, Hook. Hab.: Mexico, province of Oaxaca, at an elevation of 3,000 feet.

A. intermedium, Sicz. (A. fovearum, Raddi; A. triangulatum, Kaulf.). Hab.: West Indies: Jamaica, Cuba, Guadaloupe, Trinidad, Dominica.

A. Shepherdi, Hook. Hab.: Mexico. \$.

A. nigrescens, Fée. Hab. : Guadaloupe. \$.

A. trapeziforme, $L$. Hab. : Cordillera of Tera Cruz, Mexico ; Guatemala, Central America, Pacific side. West Indies: Jamaica, Cuba, St. Tincent.

A. polyphyllum, Willd. (A. cardiochlæna, Kunze.). Hab. : Trinidad.

A. cristatum, L. (A. Kunzeanum, Klotzsch). Hab.: West Indies : Jamaica, most abundant; Trinidad, Cuba, St. Dowingo.

A. obtusum, Desv. Hab.: Jamaica, Trinidad, St. Vincent, Bay of Choco, Panama.

A. hirtum, Klotzsch. Hab.: Panama.

A. Cubense, Hook. Hab.: Jamaica and Cuba. \$.

A. crenatum, Willd. (A. Wilesianum, Hook.). Hab.: Tabasco, Mexico. West Indies: Jamaica.

A. tetraphyllum, Willd. (A. prionophyllum, H. B. K.). Hab. : Cordillera of Oaxaca, Mexico, Vera Cruz. West Indies : Trinidad, Jamaica, Dominica, Guadaloupo.

A. lucidum, Suz. Hab. : Panama, West Indies.

A. macrophyllum, Suz. Hab.: Mexico. West Indies: Jamaica, St. Vincent, Trinidad, Guadaloupe, Cuba. Hook. S. Gr. Ic. Fil. t. 132.

A. Seemanni, Hook. Hab.: San Lorenzo, Veraguas, Central America; Guatemala. \$.

A. deltoideum, Swo. Hab.: Jamaica, on calcareous rocks; St. Domingo, crevices of rocks near the sea-shore; Cuba. $\$$.

A. villosum, L. (A. falcatum, Swz.). Hab. : Panama. W'est Indies : Jamaica, in woods; Cuba, Trinidad, St. Tincent.

A. pulverulentum, $L$. Hab.: Mexico. West Indies, frequent; Jamaica, Cuba, St. Domingo, Martinique, Guadaloupe.

A. incisun, Presl. Hab.: Guayaquil and Mexico, apparently confined to the Pacific side of Mesico; Sclanga Buba, Gorgona.

A. micropbyllum, Kaulf. Hab.: West Indies: Jamaica, Cuba, rare. $\$$.

A. Capillus-veneris, $L$. Hab.: Mexico, Guatemala; Jamaica, Trinidad, Cuba, Dominica.

A. Athiopicum, L. Hab.: Mexico, Guatemala.

A. concinmum, $H . B . K$. Hab. : Nexico and Central America. West Indies: Jamaica, Cuba, St. Vincent.

A. tenerum, Sic: Hab.: Mexico, Acapulco, and Realgo; Veraguas. West Indies; probably general : Cuba, Jamaica, Guadaloupe, St. Tincent, Buhamas, Antigua.

A. glaucophyllum, Hook. Hab.: Mexico and Guatemala; Cor- 
Adiantum-continued.

dillera of Mexico, inhabiting the cold region at an eleration of 9-10,500 feet above the level of the sea; Pic d'Orizaba, 9,750 feet. Vernguas. Hook. Cent. Ferns, t. 59.

A. fragile, Swz. Hab.: calcareous rocks, Jamaica. Hook. Cent. Ferns, t. 65 .

A. Féei, Moore. Hab. : Mexico and Guatemala. (A. Alexuosum.) Hook. Second Cent. Ferns, t. 61. \$.

A. patens, Willd. Hab.: Mexico.

A. dolosum, Kunze. Hab.: Guatemala and Panama; Jamaica, in dry plains near Bath. Grisebach.

\section{Fispolepis.}

H. repens, Presl. Hab. : Jamaica, Martinique.

H. nigrescens, Hook. Hab. : Jamaica, Martinique.

H. hostilis, Presl. Hab. : Cocos Island, Pacific, Jamaica.

H. Purdieana, Hook. Hab. . Jamaica, Westmoreland. Grisebach.

H. parallelogramma, Hook. Hab.: Guatemala.

\section{Chellanthes.}

Oh. paupercula, Mett. Hab.: Province of St. Jago, Cuba. \$.

Ch. radiata, $R$. Br. (Hypolepis radiata, Hook.). Hab. : Central America and Mexico; Guatemala. West Indies: Jamaica, Dominica, Cuba, St. Domingo, Guadaloupe, Martinique.

Ch. pedata, A. Br. (Hypolepis, Hook.). Hab.: Jamaica. \$.

Ch. microphylla, Swz. Hab.: West Indian Islands, generally; Jamaica, abundant in mountain woods; St. Vincent, Cuba, Guadaloupe, Montserrat.

Ch. allosuroides, Metten. Hab. : Mexico. \$

Cb. gracillima, Eaton. Hab.: Guatemula.

Ch. myriophylla, Desv. Hab.: Toluca, and near Oaxaca, Mexico.

Ch. scariosa, Kaulf. Hab. : said to have been gathered in Mexico.

Ch. lentigera, Suz. Hab.: Mexico, ascending to 9-11,000 feet. Real del Monte, and Pic de Orizaba.

Ch. speciosissima, Alex. Braun. Hab. : Mexico, Peak of Orizaba, ascending to 12,000 feet; Guatemala. \$.

Ch. aurea, Baker. Hab.: Guatemala.

Ch. aurantiaca, Moore (Ch. ochracea, Hook.). Hab. Mexico, moist shady places; Morelia. \$. Hook. Cent. Ferns, t. 4.

Ch. farinosa, Kaulf. Hab. : Mexico, up to 8,000 feet. Guatemala.

\section{onychium.}

\section{Ilavea.}

O. strictum, Kunze. Hab. : St. Jago de Cuba, on Mont Leban. \$.

Ll. cordifolia, Lagasca. Hab.: Mexico; Oaxaca, 5-7,000 feet; Barranca de la Encarnacion, near Zimapan; Amatenango, Chiapas; Sierra San Pedro, Nolasco, Talea. $\$$.

\section{Pellza.}

P. Seemanni, Hook. Hab.: Mazatlan, Mexico. \$.

P. geraniæfolia, Fée. Hab. : Guatemala.

P. Slkinneri, Hook. Hab.: Guatemala. \$.

P. rigida, Hook. Hab.: Mexico, Guatemala.

P. (Allosorus) ternifolia, Fée. Hab.: Mexico.

P. (Allo.) intramarginalis, J. Sm. Hab.: mountains of Mexico, Xalapa, Oaxaca, Vera Cruz, Guatemala. \$. (Cheilanthes intramarginalis, Hook. Second Cent. Ferns, t. 72.) 
Pellaa-continued.

P. (Allo.) pulchella, Fée. Hab.: high mountain regions of Mexico, elevation 7-8,000 feet, growing in tufts from the fissures of rocks south of Sola; Oaxaca, rocks near Ciudad Real.

P. (Allo.) angustifolia, Baker (Cheilanthes cuneata, Link). Hab.: Mexico; Oaxaca, 7,000 feet elevation.

P. (Allo.) glauca, J. Sm. (P. hirsuta, Hook.). Hab.: Mexicn.

P. (Allo.) marginata, Baker (Cheilanthes, $H . B . K$.). Hab.: Andes of Oaxaca; Jamaica.

P. (Platyloma) cordata, J. Smith. Hab. : Mexico.

\section{Pteris.}

Pt. Iongifolia, $L$. Hab. : Mexico. West Indian Islands : Jamaica, Cuba, Bahamas, St. Domingo, Guadaloupe.

Pt. Cretica, $L$. Hab. : Nexico and Guatemala.

Pt. mutilata, Linn. Hab, : West Indian Isles: Jamaica, Cuba, St. Domingo.

Pt. paucinervata, Fée. Hab.: Mexico, near Mirador. \$.

Pt. quadriaurita, Retz. Hab.: Mexico; Jamaica, Blue Mountains; Cuba; Trinidad.

Pt. pungens, Trilld. Hab. : West Indian Isles: Martinique and Hispaniola, Trinidad, Porto Rico.

Pt. heterophylla, L. Hab. . West Indies: Jamaica, in shady, woods; St. Domingo, Cuba.

Pt. laciniata, II $7 l d$. Hab.: Mexico; Mirador, on mountains from 600-3,000 feet of elevation. West Indian Islands : Martinique, St. Vincent, Jamaica, Guadaloupe, Dominica, Cuba.

Pt. deflexa, Link (Pt. stridens, Ag.). Hab. : Jamaica, Caraccrs.

Pt. (Prsia) aquilina, Lim. Hab.: Jamaica, Trinidad, St. Domingo, Cuba.

Pt. (Pæsia) viscosa, Baker (Pt. scalaris, Moritz.). Hab.: high mountains of Jamaica.

Pt. (Heterophlehium) grandifolia, $L$. Hab. : Mexico, Ficaltepec ad Rio Nautla. West Indies: Jamaica, Cuba.

Pt. (Campteria) biaurita, L. Hab.: West Indies, Antigua, Guadaloupe.

Pt. (Doryopteris) palmata, Willd. (Pt. pedata, L. in Hook. Sp. Fil.). Hab.: Test Indies: Jamaica, St. Domingo, Cuba, Martinique, Trinidad. Fook. Gard. Fern, t. 2.2.

Pt. (Dory.) pedata, $L$. Hab.: West Indian Islands.

Pt. (Litobrochia) denticulata, Swz. Hab.: West Indies: Cuba, St. Domingo.

Pt. (Lito.) pulchra, Schlecht. Hab. : Mexico, Tlapacoyo; Oaxaca, elevation 5,000 feet; Sierra San Pedro Nolasco, Talea, Tapilula and San Bartolo, Chiapas.

Pt. (Lito.) ciliaris, Eaton. Hab.: Cuba.

Pt. (Lito.) aculeata, Sic $\approx$ Hab. : Mexico, Tumaco. West Indies: Jamaica ; St. Vincent, most abundant.

Pt. (Lito.) incisa, Thunbg. Hab. : West Indies: Jamaica, Guadaloupe.

Pt. (Lito.) podophylla, Swa. Hab. : Mexico; Jamaica, St. Vincent, Trinidad, Cuba.

Pt. (Lito.) Kunzeana, Agardh. Hab. : Martinique. 
Pleris-continued.

Pt. (Lito.) elata, Agardh. Hab.: Panama.

Pt. (Lito.) gigantea, Willd. Hab. . West Indies: Martinique, St. Vincent.

\section{Ceratopteris.}

C. thalictroides, Brong. Hab.: quiet waters in tropical and subtropical regions; Mexico, province of Oaxaca. West Indies: Jamaica, St. Vincent, Trinidad.

\section{Xomaria.}

L. attenuata, Willd. Hab.: Guatemala; Jamaica, Martinique, St. Vincent, Cuba; Dominica.

L. L'Herminieri, Bory. Hab. . West Indies: Guadaloupe.

L. divergens, Kunze. Hab.: Martinique, Guadaloupe, Dominica.

L. onocleoides, spreng. Hab. : on trees in the interior of Jamaica ; Martinique, St. Vincent; summit of Lomodel Gobo, Cuba, climbing trees, with a caudex from 6 to 20 feet long.

L. procera, spreng. Hab.: Mexico, which appears to be its northern limit; Totutla, in thick forests, elevation 3,500-4,500 feet. Cordillera of Vera Cruz, Guatemala; Jamaica, Blue Mountain Peak; Mount Souffrière; St. Vincent, high mountains; Dominica, Couliaban Mountains; Guadaloupe.

\section{Montserrat.}

L. Boryana, Willd. (L. Magellanica, Desv.). Hab. : West Indies,

\section{Blechnum.}

B. Lanceola, Swartz. Hab.: Panama.

B. asplenioides, Sroartz. Hab. : Mexico, Panama.

B. unilaterale, Willd. (B. polypodioides, Raddi). Hab.: Mexico.

West Indian Isles: Jamaica, St. Domingo.

B. longifolium, $H . B . K$. Hab. : Trinidad, St. Vincent.

B. occidentale, Linn. Hab.: Mexico, Panama, Guatemala.

West Indian Islands : Jamaica, Dominica, St. Vincent, Cuba.

B. serrulatum, Rich. Hab.: West Indies: Dominica, Trinidad; Isthmus of Panama.

B. volubile, Kaulf. Hab. : West Indies : Dominica, Guadaloupe, Trinidad, Jamaica.

\section{woodwardia.}

W. radicans, Smith. Hab.: Mexico ; pine forests, Chiapas; Guatemala.

\section{Aspleaium.}

A. serratum, Linn. Hab.: Guatemala; West Indies, abundant.

A. fragile, Presl. Hab.: Mexico.

A. dentatum, Linn. Hab: : Mexico and Guatemala, Vera Cruz. West Indies: Guadaloupe, Jamaica.

A. Trichomanes, Linn. Hab. : Mexico, rocks 3,000 feet elevation. Guatemala, Cordillera of Orizaba, elevation 10,000 feet. Jamaica.

A. arcuatum, Liebm. Hab.: Mexico; rocks in Vera Cruz, elevation 2,000 feet; very scarce, Rincon-faisan, Vera Cruz. \$

A. monanthemum, Linn. Hab. : Mexico, very abundant; Guatemala, Realejo.

A. ebeneum, Ait. Hab. : Mexico; West Indies.

A. alatum, H. B. K. Hab. : West Indies: Jamaica. St. Vincent. 
Asplenium-continued. A. salicifolium, Linn. Hab.: Panama. West Indies: Cuba,
Jamaica.

A. virens, Presl. Hab. : Gorgona Island and Guayaquil.

A. erectum, Bory. Hab.: Mexico, Guatemala. West Indies : Cuba, St. Vincent, Jamaica ; Trinidad.

A. obtusifolium, L. (A. riparium, Liebm.). Hab.: Mexico, damp rocks and waterfalls; Xalapa; Martinique, Guadaloupe; Dominica, in moist places.

A. firmum, Kunze. Hab. : Guatemala; Cuba, Jamaica.

A. cultrifolium, Linn. Hab.: Guatemala. West Indies: Jamaica, Cuba.

A, auriculatum, Swartz. Hab.: Mexico. West Indies: Jamaica, GuadaIoupe, Dominica, St. Vincent, Cuba.

A. anisophyllum, Kunze. Hab.: Cuba.

A. rhizophorum, Limn. Hab.: Nexico, Oaxaca. West Indies : Guadaloupe, Porto Rico, Cuba.

A. Serra, Langs \& Fisch. Hab.: Mexico. West Indies : Jamaica, Dominica, Cuba, Guadaloupe.

A. marinum, Linn. Hab.: St. Vincent; Jamaica, on the rocky coast near Bath.

A. auritum, Szvartz. Hab.: Mexico, Guatemala, Panama, and Isle of Gorgona. West Indies: Jamaica, Dominica, Cuba.

A. erosum, Linn. Hab. : West Indies: Jamaica, Portland Gap, Cuba. $\$$

A. obesum, Baker. Hab.: Guatemala. \$.

A. dimidiatum, Swartz. Hab. . West Indian Islands : Jamaica, Cuba.

A. formosum, Willd. Hab. : Mexico, Guatemala, Cuba.

A. lætum, Sw: Hab.. Mexico and Panama; abundant in the West Indian Islands, Jamaica, Trinidad, St. Vincent, Guadaloupe, Dominica, Cuba.

A. bissectum, Sivz. Hab.: West Indies: Jamaica, Cuba.

A. pumilum, Suz. Hab.: Mexico, Orizaba, San Blas. West Indies : Jamaica, Martinique, Cuba, St. Yincent, Guadaloupe.

A. cuneatum, Lam. Hab.. West Indies: Jamaica, Cuba, St. Vincent; Trinidad; Guadaloupe.

A. furcatum, Thunb. IIab. : Guatemala, Mexico, on Liquidamber trees of Xalapa. West Indies: Jamaica, on Catherine Peak, eleration 5,000 feet.

A. fragrans, Swz. Hab.: Mexico ; Oaxaca, 5,000 feet elevation; Mirador, Veraguas. West Indies : Jamaica, Blue Mountain Pealr, Portland; Cuba, Guadaloupe, Dominica.

A. adiantoides, Raddi. Hab.: Jamaica.

A. squamosum, Linn. Hab.: St. Domingo, rare.

A. bulbiferum, Forst. Iab. : Mexico; Oaxaca, elevation 5,000 feet; Guatemala.

A. Fadyeni, Hook. Hab. : Jamaica. Hook. Second Cent. Ferns, t. 27 . \$5.

A. rhizophyllum, Kunze. Hab. : Mexico. West Indies: Jamaica, Dominica, Porto Rico, Trinidad, Cuba.

A. cicutarium, Swoz. Hab. : Mexico, Boqueta, Veraguas, and Isle of Gorgona. West Indies: Jamaica, abundant; Cuba, Porto Rico, Martinique, rocky woods in Guadaloupe. Fée. 
Asplenium-continued.

A. (Athyrium) Skinneri, Baker. Hab.: Guatemala. \$.

A. (Athyr.) achillæfolium, Liebm. Hab.: Mexico, Mirador, Volcan de St. Martin, Vera Cruz; Guatemala. \$.

A. (Athyr.) Filix-fomina, Bernh. Hab.: Cuba.

A. (Athyr.) conchatum, Moore. Hab.: St. Domingo, Jamaica, Cuba. \$.

A. (Diplazium) plantagineum, Linn. Hab. : Mexico, 4,000 feet elevation; Guatemala. West Indies: Jamaica, Guadaloupe.

A. (Dipl.) Seemannii, Baker. Hab.: Darien. \$.

A. (Dipl.) Callipteris, Baker. Hab.: Cuba, Guadaloupe.

A. (Dipl.) grandifolium, Swz. Hab.: Jamaica, Trinidad, Martinique, Cuba.

A. (Dipl.) Alavescens, Mett. (A. juglandifolium, Lam.). Hab. : Jamaica, Cuba.

A. (Dipl.) celtidifolium, Kunze. Hab.: Jamaica.

A. (Dipl.) arboreum, Willd. (D. auriculatum, Kaulf.). Hab.: West Indies: St. Vincent; Jamaica, 'Cold-spring Gap;' Cubr.

A. (Dipl.) Shepherdi, Spreng. (A. striatum, Linn.). Hab. : Mexico, Vera Cruz, Tabasco. West Indies: Cuba, Dominica, St. Vincent, Barbadoes; Jamaica.

A. (Dipl.) semihastatum, Kze. Hab. : Cuba; Jamaica.

A. (Dipl.) crenulatum, Baker. Hab.: Mexico, Cuba.

A. (Dipl.) Lind bergii, Mett. Hab.: Mexico.

A. (Dipl.) costale, Swartz. Hab.: West Indies, Jamaica.

A. (Dipl.) Franconis, Mett. Hab.: Mexico, Oajaca, Guatemala; Jamaica.

A. (Dipl.) hians, Kunze. Hab.: Jamaica.

A. (Dipl.) radicans, Schk. Hab.: Mexico, Tabasco, Jamaica, St. Vincent, Guadaloupe, Martinique, Cuba.

A. (Dipl.) Wilsoni, Baker. Hab.: Jamaica. \$. $\$$.

A. (Anisogonium) ternatum, Hook. Hab.: Mexico, Oaxaca.

A. (Hemidictyum) marginatum, Linn. Hab. : West Indies; one of the finest and most common of Tropical American Ferns, on the borders of streams, and in damp, moist, shady woods.

\section{Scolcpendrium.}

S. vulgare, Sm. Hab. : Chiapas, Mexico.

S. (Schaffneria) nigripes, Hook. Hab. : Mexico, Guatemala. \$\$.

\section{Didymochlana.}

D. lunulata, Desv. Hab.: Guatemala. West Indian Islands: Trinidad, Cuba.

\section{Aspidium.}

A. (Polystichum.) glandulosum, Hook. \& Grev. Hab.: Cuba, east side of the island; Jamaica, above the old Botanic Garden, rare. When fresh gathered, very viscid. Hook. \& Gr. Ic. Fil. t. 140 . \$

A. (Polyst.) Plaschnichianum, Kunze. Hab.: Jamaica, wet shady places; St. George's Parish, rare. \$.

A. (Polyst.) rhizophyllum, Swz. Hab.: Jamaica and Cuba. Hook. \& Gr. Ic. Fil. t. 59 . \$.

A. (Polyst.) semicordatum, Shoz. Hab.: West Indian I3lands, probably universal; Jamaica, St. Vincent, Trinidad, Cuba. 
Aspidium-continued.

A. (Polyst.) mucronatum, Swoz. IIab. : West Indies: Jamaica, Port Royal Mountains, St. Domingo, Guadaloupe. \$.

A. (Polyst.) triangulum, Su. Hab. . West Indies: Jamaica (Cedar Vailey, St. George), Cuba. \$.

A. (Polyst.) viviparum, Fée. Hab.: West Indies: Cuba, Jamaica. $\$$.

A. (Polyst.) tridens, Hook. Hab.: Jamilica, rare; near Woburn I awn, Port Royal; Arntilly Gap, Blue Lountains, elevation 3,000 feet. $\$$.

A. (Polyst.) aculeatum, Swz. Hab.: Mexico, Guatemala. West Indian Islands: Jamaica, Trinidad.

A. (Polyst.) Capense, Willd. (A. coriaceum, Swo.). Hab.: West Indies: Jamaica, on rocks; Cuba.

A. (Polyst.) Seemanni, Hook. Hab. : Panama, Bay of Ardita. \$\$.

A. (Polyst.) ascendens, Hew. Hab.: Jamaica; mountain forests about 'old English plantation,' Manchester, climbing 20-30 feet up the trunks of trees; also from Woodside, St. Mary's, and from Moncaque; very rare. $\$$.

A. (Polyst.) melanostictum, Kzc. Hab.: Mexico, Xalapa. \$.

A. (Polyst.) melanochlanys, Fée. Hab.: Cuba; Mont Liban, near the town of Monte Verde. $\$$.

A. (Cyrtomium) abbreviatum, Schrad. Hah.: West Indies: Jamaica; Trinidad, in wet places; Aripo. Grisebach.

A. (Cyrt.) juglandifolium, Kze. Hab.: Mexico.

A. (Cyclodium) meniscioides, Willd. Hab.: Trinidad, in damp woods; Aripo. Hook. \& Gr. Ic. Fil. t. 12l. (Aspidium confertum.)

A. plantagineum, Griseb. Hab.: Panama. West Indies: Martinique, St. Vincent; Dominica, in mossy rocky places near water.

A. trifoliatum, Sws. Hah. : Panama, Guatemala, Mexico, Vera Cruz. West Indies: Jamaica, Cuba, Bahamas, Martinique, Dominica, Guadaloupe, St. Vincent; Barbadoes.

\section{Tephrodium.}

N. (Lastrea) pedatum, Hook. Hab. . West Indies: Cuba, Jamaica. \$.

N. (Last.) patens, Desv. Hab.: Mexico, Guatemala. W' West Indian Isles: Jamaica, Guadaloupe, Dominica, Cuba, Bahamas, St. Vincent.

N. (Last.) invisum, Baker (N. macrourum and Serra, Hook.). Ilab.: Mexico. West Indies: Cuba; Jamaica, common in woods; Dominica, Trinidad. Grisebach.

N. (Last.) falciculatum, Desw. Hab. : Mexico.

N. (Last.) velleum, Baker (N. aureo-vestitum, Hook.), Hab. . West Indies: Mont Leban, St. Jago de Cuba, Jamaica.

N. (Last.) Caripense, Hook. Hab.: Guatemala.

N. (Last.) trichophorum, Baker. Hab.: Guadaloupe.

N. (Last.) triste, Hook. Hab. : Mexico, Calipa.

N. (Last.) sanctum, Baker. Hab.. Guatemala. West Indies: Jamaica, on rocks; Cuba, Guadaloupe. \$.

N. (Last.) conterminum, Desv. Hab.: Mexico. West Indies : Jamaica, Blue Mlountains, to 7,000 feet alt.; Dominica, Guadaloupe, Martinique, Porto Rico, Cuba. 
Nephrodium-continued.

N. (Last.) Kaulfussii, Hook. Hab,: West Indies.

N. (Last.) concinnum, Baker. Hab. : Mexico.

N. (Last.) Sprengelii, Hook. Hab.: Guatemala. West Indies : Jamaica, Martinique, Porto Rico, Antilles, St. Vincent. \$.

N. (Last.) limbatum, Desv. Hab.: West Indies: Jamaica, St. Vincent, Guadaloupe, St. Kitt's. \$.

N. (Last.) velatum, Hook. Hab.: Cuba. \$.

N. (Last.) lonchodes, Hook. Hab. : Cuba, near Monte Verde. \$\$.

N. (Last.) deltoidenm, Desv. Hab.: West Indies, probably in most of the islands; Jamaica, in mountain woods; Guadaloupe, Cuba, Porto Rico. \$

N. (Last.) Filix-mas, Rich. Hab. : Mexico and Guatemala.

N. (Last.) Salvini, Baker. Hab. : Guatemala. \$.

N. (Last.) mexicanum, Hook. Hab. : Mexico, Panama, Jamaica.

N. (Last.) sphærocarpum, Hook. Hab.: Mexico.

N. (Last.) hirtum, Hook. Hab. : mountains of Vera Paz, Guatemala; Jamaica, on moist shady rocks.-Grisebach. Cuba.

N. (Last.) Karwinslkyanum, Baker. Hab.: Mexico and Guatemala. $\$$.

N. (Last.) pubescens, Desv. Hab.: West Indies: Jamaica, Cuba, Porto Rico, Guadaloupe. \$\$.

N. (Last.) subquinquefidum, Hook. (N. funestum, Hook.). Hab.: West Indies, Porto Rico, Guadaloupe, Trinidad.

N. (Last.) Grisebachii, Baker. Hab. : Cuba. \$\$.

N. (Last.) amplum, Baker (Polyp. Sloanei, Kze.). Hab.: Guatemala; Volcan de Fuego, alt. 7,000 feet; Cuba, St. Vincent, Dominica; abundant in Jamaica; Trinidad.

N. (Last.) villosum, Presl. Hab. : Jamaica, rare; in moist woods, at 3-4,000 feet alt. ; Cold-spring Plantation ; Fort Steward, Bluefield Mountains, Cuba.

N. (Last.) denticulatum, Hook. Hab.: Guatemala, Jamaica, Cuba.

N. (Last.) effusum, Baker. Hab.: Mexico, Jamaica, Cuba; St. Vincent.

N. Cumingianum, J. Sm. Hab.: Panama. \$.

N. Skinneri, Hook. Hab. : Guatemala, Hook. Cent. Ferns, t. 2 t.

N. Wrightii, Hook. Hab. : dense woods near Monte Verde, Eastern Cuba. \$.

N. scolopendrioides, Hook. Hab.: West Indies: Jamaica, in dry places on rocks; St. Domingo, Guadaloupe, Cuba.

N. incisum, Baker (N. stenopteris, Hook.). Hab. . West Indies: Cuba; Jamaica, in woods; Bahamas. Grisebach.

N. varians, Fée. Hab.: Trinidad. \$.

N. unitum, $R$. $\boldsymbol{B r}$. (N. propinquum, Br.). Hab.: Mexico; Jamaica, in morasses ; Guadaloupe.

N. Serra, Desv. Hab. : Jamaica, in wet places; St. Ann's, St. Mary's, and Cuba. \$s.

N. asplenioides, Baker (N. sclerophyllum, Prest.). Hab. : West Indies: Jamaica, St. Vincent, Trinidad, Cuba. \$.

N. molle, Desv. Hab.: Mexico. West Indian Islands, probably universal; Jamaica, St. Vincent, Cuba, Guadaloupe.

N. venustum, J. Sm. Hab.: Jamaica, Guadaloupe. \$. 
Nephrodium-continued.

N. brachyodon, Hook. Hab.: Panama, various parts of the coast. West Indies: St. Vincent, Dominica.

N. (Pleocnemia) dissidens, Hook. Hab.: Porto Rico. \$.

N. (Sagenia) subtriphyllum, Baker. Hab.: Panama.

N. (Sagen.) latifolium, Baker. Hab.: Panama.

N. (Sacen.) cicutarium, Baker. Hab. . Mexico, west coast of Panama; Guatemala. Probably abundant in all the West Indian Islands; Jamaica, in mountain woods; Trinidad, Cuba, Porto Rico.

N. (Sagen.) macrophyllum, Baker (aspid., \$w.). Hab. : Mexico and Panama; Martinique, St. Vincent, Jamaica, Cuba, Dominica, Trinidad; probably in all the West Indian Isles.

\section{Nephrolepis.}

N. cordifolia, Baker (N. tuberosa, Pr.). Hab.: West Indies: Jamaica, Mexico.

N. exaltata, Schott. Hab. : Mexico ; Cuba, Jamaica, St. Vincent, the Bahamas; Martinique, Guadaloupe.

N. acuta, Presl. Hab.: Guatemala, Panama, Cuba, Jamaica, Dominica.

oleandra.

O. nodosa, Presl. Hab.: West Indies, frequent: Cuba; Jamaica, on decayed trees; Martinique, St. Vincent, Trinidad, Dominica, Guadaloupe.

\section{radyenia.}

F. prolifera, Hook. Hab.: Cuba and Jamaica, high and shady woods. \$.

\section{Polypodium.}

P. (Phegonteris) cordatum, Hook. Hab.: Jamaica, on limestone rocks; Cuba.

P. (Pheg.) hastrfolium, Sw. Hab. . W. Indies: Jamaica, in shady woods and on rocks; Cuba. $\$$.

P. (Pheg.) Havo-punctatum, Laulf. Hab.: Mexico, Martinique, Guadaloupe, Dominica, Jamaica, St. Vincent.

P. (Pheg.) Germanianum, Baker. Hab. : Guadaloupe. \$.

P. (Pheg.) rusticum, Baker. Hab.: Guadaloupe. \$.

P. (Pheg.) refulgens, Klotwsch. IIah. : Panama.

P. (Pleg.) rude, Kunze. Hab. : Mexico.

P. (Pheg.) decussatum, $L$. Hab.: Panama, abundant in the West Indian Islands; Jamaica, in moist mountain woods, at 3,000 feet alt. Nontserrat, St. Vincent. Grisebach.

P. (Pheg.) caudatum, Kaulf. Hab. : Cuba, Jamaica.

P. (Pheg.) glanduliferum, Liebm. Hab.: Mexico; Oaxaca, alt. 2,500 feet. $\$$.

Jamaica.

P. (Pheg.) platyphyllum, Hook. Hab.: Guatemala, Cuba,

P. (Pheg.) rigidum, Hook. \& Grev. Hab. : Guatemala.

P. (Goniopteris) meniscioides, Liebm. Hab.: Itexico. \$.

P. (Goniopt.) crenatum, Swoz. Hal,. Mexico; frequent in the W'est Indian Islands, Cuba, Jamaica, St. Tincent, Trinidad. $\$$.

P. (Goniopt.) Gheisbreghtii, Linden. Hab. : Tabasco, S. Mexico,

P. (Goniopt.) fancium, Liebm. Mab.: Mexico. \$s.

P. (Goniopt.) reptans, Swz. Hab. : Trest Indies: Jamaica, Cubs.

P. (Goniopt.) tetragonum, Swz. Hab.: abundant in the West 
Polypodium-continued.

Indies: Jamaica, Dominica, St. Vincent, Trinidad, Martinique, Guadaloupe, Panama.

P. jungermannioides, Klotzsch. Hab.: Guatemala.

P. (Grammitis) gramineum, Swz. Hab.: Jamaica, on trees in the Blue Mountains; St. Vincent.

P. (Gram.) marginellum, Swz. Hab.: Jamaica, on trees, Blue Mountain Peak; Guadaloupe, Guatemala.

P. trifurcatum, L. (P. comptoniæfolium, Desv.). Hab.: West Indies: Martinique, Guadaloupe, Porto Rico. (P. scolopendrioides, Hook. \& Gr. Io. Fil. t. 42.)

P. serrulatum, Mett. (Xiphopteris Jamesoni, Hook.). Hab.: Mexico. West Indies: Jamaica, summit of the Blue Mountains; Dominica, St. Vincent, Trinidad. Hook. Second Cent. Ferns, t. 14.

P. inæquale, Fée. Hab.: Guadaloupe.

P. moniliforme, Lagasc. Hab.: Guatemala ; Mexico, 9-12,000 feet; Jamaica, Cuba. (Jamesonia adnata, Kze.)

P. exiguum, Griseb. Hab. : Jamaica. \$.

P. trichomanoides, Swz. Hab.: Cuba.

P. delicatulum, Mart. \& Gal. Hab. : Mexico, on oaks of the Sierra Yavergia, elevation 7,000 feet.

P. jubæforme, Kaulf. Hab. : Panama. West Indies: Porto Rico, Antilles, St. Vincent. \$.

P. pilosissimum, Mart. et Gal. Hab. : Mexico.

P. cultratum, Willd. Hab.: Guatemala, Cuba, Martinique.

P. decipiens, Hook. Hab.: Jamaica.

P. curvatum, Swz. Hab. . Jamaica, on trees near the mountain peaks; Guadaloupe.

P. pendulum, Swz. Hab. : on trees in the mountains, West Indies: Jamaica, Guadaloupe.

P. suspensum, L. Hab.: Mexico; Guatemala. West Indies: Jamaica, Martinique, St, Vincent, Cuba. $\$$.

P. griseum, Liebm. Hab. . Mexico, Dep. of Puebla; Guatemala.

P. rigescens, Bory. Hab. : Cuba. Hook. \& Gr. Ic. Fil. t. 216.

P. Skinneri, Hook. Hab. : Guatemala.

P. furfuraceum, Schlecht. Hab.: Mexico, Mirador. \$.

P. pulchrum, Mart. \& Gal. Hab.: Mexico, Xalapa, elevation 4,000 feet; Cuba.

P. taxifolium, $L$. (P. Plumula, $H . B . K$.). Hab.: Mexico. West Indies: Jamaica, St. Domingo.

P. pectinatum, L. Hab. : Mexico. West Indies: Jamaica, Dominica, Cuba.

P. ellipsoideum, Fée. Hab. : Mexico. \$.

P. Martensii, Mett. Hab. : Mexico; Orizaba, 9-10,000 feet; Xalapa and Real del Monte. \$.

P. tenuifolium, H. B. K. Hab.: West Indies: Jamaica, Cuba, St. Domingo.

P. vulgare, $L$. Hab. : north of Mexico.

P. heteromorphum, Hook. et Grev. Hab. . Mexico.

P. plebeium, Schlecht. Hab. : Mexico, on mountains, alt. 311,000 feet; Guatemala, Vera Paz.

P. Hartwegianum, Hook. Hab. : Mexico, on the mountain Sumata, elevation 9,500 feet. $\$$. 
Polyporlium-continued.

P. puberulum, Schlecht. Hab.: Mexico and Guatemala.

P. subpetiolatum, Hook. Hab.: Mexico, Cordillera of Oaxaca, Cuba. \$.

P. sororium, H. B. K. Hab. : Mexico, Cuba.

P. fraternum, C. \& S. Hab.: Mexico. \$\$.

P. legionarium (P. macrodon, Hook, non Reinvo.). Hab. : Cohar and Vera Paz; Guatemala.

P. fa]lax, Schlecht. Hab.: Mexico, Vera Cruz; Puebla, on mountains $4-7,000$ feet alt. $\$$.

P. funiculum, F́ce. Hab. : Cuba.

P. Friedrichsthalianum, Kze. Hab.: Guatemala, Vera Paz, Mexico, Chiapas,

P. (Goniophlebium) vaccinïfolium, Fisch \& Langd. Hab.: Jamaica.

P. (Conioph.) piloselloides, $L$. Hab.: West Indies.

1. (Gonioph.) patens, $J$. Sm. Hab. : Panama. $\$$.

P. (Gouioph.) plesiosorum, Kunze (P. loriceum, L. in part). Hab. : Mexico, Guatemala.

P. (Gonioph.) loriceum, L. (P. Catherinæ, J. Sm.). Hab. : Mexico. West Indies: Cuba, Jamaica, Dominica, St. Vincent, Martinique, Guadaloupe.

P. (Gonioph.) Surrucuchense, Hook. Hab.: Jamaica, Dominica.

P. (Gonioph.) plectolepis, Hook. Hab.: Totutla, Mexico, Grtatemala. \$\$.

P. (Gonioph.) chnoodes, Spreng. (P. dissimile, Hook.). Hab. : West Indies, frequent; Jamaica, Dominica, St. Vincent; Guadaloupe, in damp forests, on trees and rocks; Martinique.-Fée. Trinidad, on cocoa-trees.

P. (Gonioph.) attenuatum, $H . B . K$. Hab.: W. Indies.

P. (Gonioph.) neriifolium, Schk. Hab.: West Indies: Jamaica, Dominica, Trinidad.

P. (Gonioph.) Guatemalensis, Hook. Hab.: Guatemala. \$.

P. (Gonioph.) adnatum, Kunze.' Hab.: Guatemala.

P. (Goniopb.) menisciifolium, Langsd. \& Fisch. Hab. : Panama, on the trunk of an oil-palm; T'rinidad.

P. (Cronioph.) incanum, Sroz. Hab.: Mexico. West Indies: Jamaica, on trees; St. Vincent, Trinidad, Cuba.

P. (Cronioph.) lepidopteris, Kze. Hab. . Panama, Mexico; Vera Cruz, on oaks ; 3,500-5,000 feet.

P. (Gonioph.) squamatum, L. Hab.: Mexico, apparently frequent on mountains, alt. 7,000 feet.

P. (Phlebodium) aureun, L. Hab.: Mexico, Cuba, St. Vincent, Martinique; Jamaica, on trees.

P. (Campyloneuron) angustifolium, Swz. Hab.: Mexico: Jamaica, on dry banks and trees. - Grisebach. Cuba, almost universal in Tropical America.

P. (Camp.) lapathifolium, Lam. (P. fasciale, Willd.). Hab.: Guatemala ; Jamaica, Cuba.

P. (Camp.) repens, $L$. Hab. : Mexico, West Indies.

P. (Camp.) Phyllitidis, L. Hab. . Cuba, Jamaica, St. Tincent. 'Tropical America, chiefly, I believe, confined to the Atlantic side, and the Carribean Seas.' Hook. Sp. Fil. 
Polypodium-continued.

P. (Phymatodes) lanceolatum, Linn. (P. lepidotum, Willd.). Hab.: Panama ; Mexico, frequent; Jamaica, common on dry rocks. P. (Phym.) Swartzii, Baker (P. serpens, Swz.). Hab.: Martinique, Cuba.

P. (Phym.) lycopodioides, Linn. Hab.: Mexico. West Indies: Cuba, Jamaica, Dominica.

P. (Phym.) persicariæfolium, Schrad. Hab. : Guatemala. West Indies : T'rinidad.

P. (Phym.) crassifolium, Linn. Hab.: Mexico. West Indies: Jamaira, Dominica, St. Vincent, Trinidad, Cuba.

P. (Phym.) crispatum, Hook. Hab.: Isle de Cacagual, Southern Darien and Bay of Choco, Panama. \$. Indies.

P. (Phym.) angustum, Mett. Hab.: Mexico, Guatemala, West

\section{mothochlæena.}

N. sinuata, Krulf. Hab.: Mexico, from east to west, apparently abundant.

N. ferruginea, Hook. (N. rufa, Pr.). Hab.: Guatemala and Mexico, alt. 2-5,000 feet; Jamaica, on rocks and shingly soil, St. Andrew's parish, and elsewhere, frequent; alt. 3-4,000 feet. N. Aschenhorniana, Kze. Hab. : mountains of Mexico, 5-8,000
feet. $\$$.

N. squamosa, Baker (Cheilanthes, Hook.).Hab.: Mexico and Guatemala. Hook. \& Gr. t. 151. \$.

N. mollis, Kunze. Hab.: Andes of Guatemala.

N. (Cincinalis) trichomanoides, $R$. Br. Hab. . Jamaica, common, and Cuba. \$.

N. (Cinc.) affinis, Hook. Hab.: Mexico and Guatemala. \$.

N. (Cinc.) sulphurea, J. Sm. (N. candida, Hork.). Hab. : Mexico.

N. (Cinc.) flavens, Moore (Gymnogramme flavens, $K l f s$.). Hab.: Central America. $\$$

N. (Cinc.) nivea, Desv. Hab.: Mexico.

\section{Monogramme.}

M. rostrata, Hook. Hab. : island on the Lake of Omotepec, Nicaragua.

M. graminioides, Baker (M. furcata, Desv.). Hab.: Jamaica, on trees in the highest mountains; Cuba. Grisebach.

M. (Pleurogramme) seminuda, Baker (M. graminifolia, Hook.). Hab. : Guatemala. West Lndies: Dominica, St. Vincent, Trinidad, Guadiloupe.

M. (Pleur.) immersa, Fée. Hab.: Cuba.

\section{Gymnogramme.}

G. (Leptngramme) pilosa, Mrrt. \& Gal. Hab. . Mexico. \$.

G. (Lept.) asplenioides, Kaulf. Hab. : Panama.

G. (Lept.) diplazioides, Desv. Hab. : Mexico.

G. (Lept.) gracilis, Hewarl. Hab.: Jamaica, mountains of Port Royal, Westmoreland, Guadaloupe. \$.

G. (Lept.) pumila, Spreng. Hab. : Jamaica, Trinidad.

G. rufa, Desv. Hab.: Panama, Guatemala; Jamaica, in dry hot plains; Cuba. 


\section{Gymnogramme-continued.}

G. pedata, Kaulf. Hab. : Mexico, Guatemala.

G. leptophylla, Desv. Hab. : Andes of Mexico.

G. chærophylla, Desv. Hab. . Guatemala, Cuba and Jamaica. Hook. \& Grev. Ic. Fil. t. 45.

G. fléxuosa, Desv. Hab. : Nicaragua.

G. (Ceratopteris) trifoliata, Desv. Hab. : Guatemala, Cuba ; Jamaica, on river sides.

G. tartaren, Desv. (G. ornithopteris, Kl.). Hab. : Jamaica, St. Vincent, Cuba.

G. (Cerop.) calomelanos, Kaulf. Hab. : West Indies: Jamaica, Cuba, Dominica, Guadaloupe, St. Domingo.

G. (Cerop.) sulphurea, Desv. Hab. . West Indies: Cuba; Jamaica, abundant, on wet shady banks, at Antilly Gap, alt. 2,500 feet.

G. (Selliguea) Mexicana, Baker (G. Salvinii, Hook.). Hab. : Mexico and Guatemala; Vera Paz, alt. 3,500-5,000 feet. Hook. Second Cent. Ferns, t. 71.

G. (Sell.) elongata, Hook. Hab.: Cuba and throughout the West Indies.

G. (Sell.) podophylla, Hook. Hab. : Mexico, between Vera Cruz and Orizaba.

\section{Meniscium.}

M. angustifolium, Willd. Hab.: Panama. West Indies : Jamaica, on stones in river courses; Cuba. dad.

M. serratum, Cav. Hab. : Mexico, Panama West Indies: Trini-

M. reticulatum, Sw. Hab.: Mexico. West Indian Islands: Dominica, St. Vincent, Trinidad.

\section{Antrophyum.}

A. lineatum, Kaulf. Hab. : Jamaica, on trees; St. George's.Grisebach. Cuba; Irexico, Guatemala, Vera Paz.

A. lanceolatum, Krulf. Hab.: Mexico, Guatemala, Vera Paz. West Indies: Jamaica, on fallen trees ; Dominica, St. Vincent, Barbadoes, Cuba. Grisebach.

A. ensiforme, Hook. Hab. : Mexicn, alt. 10.000 feet; Guatemala, and on mountains of Vera Paz, alt. 3,500-5,000 feet. Hook. Second Cent. Ferns, t. 70.

A. subsessile, Kunze. Hab.: Guatemala, Cuba.

\section{Vittaria.}

T. (Tæniopsis) scabrida, Klotzsch. Hab. : Mexico.

V. (Tæniop.) lineata, Sw. Hab.: Nicaraqua, Mexico, Cuba ; Jamaica, common on trees; St. Vincent, Barbadoes.

V. (Tæuiop.) remota, Fée. Hab.: West Indies.

\section{Trenitis.}

T. angustifolia, R. Br. Hab.: West Indian Islands, abundant; Jamaica, on locks and trees; Cuba, St. Vincent, Trinidad.

T. lanceolata, $R$. Br. Hab. : Guatemala. West Indian Islands, frequent; Martinique; Jamaica, on trees; Cuba, Guadaloupe. \$.

T. furcata, Willd. Hab. : Panama. W. Indies: Cuba; Trinidad, on trees, frequent. 


\section{Eemlonitis.}

H. palmata, Linn. Hab. : Mexico, Guatemala; Jamaica, Cuba, Dominica, Trinidad, Martinique.

H. hederæfolia, J. Sm. Hab. : Mexico. \$.

H. pinnatitida, Baker. Hab.: Central America. \$.

H. pinnata, J. Sm. Hab. : Jamaica. \$.

H. (Anetium) citrifolia, Hook. Hab.: common thronghout the West Indies and tropical America, frequently growing pendulous from palm stems; Jamaica, St. Vincent, Trinidad, Porto Rico.

\section{Acrostichum.}

A. simplex, Sw. Hab. : Jamaica, on decayed trees; St. Vincent, Cuba, Guadaloupe.

A. conforme, Sw. Hab.: Panama; Jamaica, on decayed trees; Dominica, on Mount Couliaban.

A. Wrightii, Mett. Hab. . Cuba. \$.

A. flaccidum, Fée. Hab.: Panama.

A. Schlimense, Fée. (A. scaudens, Bory.). Hab. : Guadaloupe.

A. Herminieri, Bory. Hab.: Guadaloupe, Cuba.

A. Lingua, Raddi (A. latifolium, Sw. in part.). Hab.: West Indies : Cuba, Dominica, Jamaica, Guadaloupe.

A. luridum, Fée. Hab.: Trinidad, in woods; Arima. Grisebach. A. latifolium, Swo. Hab. : Mexico, Cuba; Jamaica, on trees; St. George"s, Dominica, St. Vincent. Grisebach.

A. hybridum, Bory. Hab. . Mexico.

A. decoratum, Kae. Hab.: West Indies, Guadaloupe.

A. Féei, Bory. Hab. : Guadaloupe; on Mount Couliaban, Dominicr. Hook. Second Cent. Ferns, t. 92.

A. Lindeni, Bory. Hab. : Mexico.

A. Aubertii, Desv. Hab. : Guatemala.

A. viscosum, Sav. Hab.: Mexico. West Indies: Jamaica, Dominica, St. Vincent, Cuba, Guadaloupe, \&c.

A. pilosum, H. B. K. Hab. : Mexico.

A. apodum, Kanlf. Hab.: West Indies : Montserrat, St. Vincent ; Jamaica, in woods near Bath; Cuba.

A. scolopendrifolium, Raddi. Hab.: Guatemala.

A. Boryanum, Fée. Hab.: West Indies: Guadaloupe, Martinique; Dominica, Couliaban Mountains; Trinidad.

A. ovatum, Hoak. Hab. : Mexico.

A. procurrens, Mett. Hab.: Cuba. \$.

A. spathulatum, Bory. (A. piloselloides, Pr.). Hab. . Cuba and West Indies generally.

A. lepidotum, Willd. (A. vestitum, Schlecht.). Hab.: Jamaica, on trees, Blue Mountains. Grisebach.

A. villosum, Sicz. Hab. . Mexico, Jamaica, Dominica, Cuba, Guadaloupe.

A. Matthewsii, Fée. Hab.: Mexico.

A. tectum, Willd. (A. rubiginosum, Fée.). Hab. : Mexico, Guatemala, Jamaica, Dominica.

A. muscosum, Sivz. (A. Langsdorfii, Hook. \& Gr.). Hab. . Mexico, Dominica, Jamuica, Blue Mountain Peak, St. Domingo.

A. auricomum, Kunze. Hab. : Mexico.

A. cuspidatum, Willd. Hab. : West Indies. 
Acrostichum-continued.

A. perelegans, Fée. Hab.: Couliaban Mountains, Dominica, St. Domingo, Martinique.

A. squamosum, Swz. Hab. : Mexico ; Jamaica, St. George's, near Springhill, frequent.

A. (Stenochlæna) sorbifolium, L. Hab.: West Indies: Cubs, Jamaica, Montserrat, St. Vincent, Dominica ; Trinidad, in woods, on trees.

A. (Polybotrya) aspidioines, Baker. Hab. : Cuba. \$.

A. (Polyb.) caudatum, Hook. Hab. : Mexico, Panama, Trinidad; Woodford, on trees.

A. (Polyb.) osmundaceum, Hook. Hab.: Jamaica, near Fox's Gap, twining up the trees; Cuba.

A. (Rhipidopteris) peltatum, Swz. Hab.: Mexico. West Indies : Jamaica, on trees and rocks, St. George's; Dominica, Cuba.

A. (Olfersia) cervinum, Swz. Hab.: Mexico, Cuba; Jamaica, in woods; Dominiea, St. Vincent; Trinidad, on rocks.

A. (Soromanes) Coenopteris, Kze. Hab. : Mexico.

A. (Gymnopteris) alienum, Swz. Hab.: Mexico, Guatemala, Cuba; Jamaica, in moist woods ; St. James, Manchioneal ; Trinidad. Grisebach.

A. (Gym.) nicotiangefolium, Swz. Hab.. Cuba; Jamaica, in moist woods, Manchester. Grisebach.

A. (Chrysodium) crinitum, Linn. Hab, : Mexico. West Indies, frequent; Jamaica, on rocks, in shady woods, St. George's; Dominica, St. Vincent, Cuba. $\$$.

A (Chrys.) prestantissimum, Bory. Hab.: West Indies: Guadaloupe, Dominica. \$.

A. (Chrys.) aureum, $L$. Hab. : Jamaica, in marshes and bogs.

\section{Osmunda.}

O. cinnamomea, Linn. Hab. : Mexico, Guatemala, West Indies.

\section{Schizeea.}

S. (Lophidium) dichotoma, Swz. Hab.: Cuba.

S. (Loph.) elegans, Swz. Hab. : Mexico. West Indies : Jamaica, very rare, in dry woods, Westmoreland, Bluefield Mountains, at 2,000 feet alt.; Trinidad. Grisebach; Hook." Gard. Ferns, t. 54 .

S. (Actinostachys) pennula, Swz. Hab.: West Indies: Guadaloupe, Trinidad.

\section{Anemia.}

A. filiformis, Presl. Hab.. Mexico. West Indies: Jamaica.

A. oblongifolia, Swz. (A. humilis, Swz.). Hab.: Mexico; Jamaica. bach.

A. mandioccana, Raddi. Hab.: Mexico, Jamaica, Cuba. Grise-

A. Breuteliana, Presl. Hab. : Mexico. West Indies: St. Kitts, St. Domingo, Trinidad.

A. Mexicana, Klotzsch. Hab. : Guatemala. Hook. Cent. Ferns, t. 88. (Var. paucifolia.) Hook. Secund Cent. Ferns, t. 65.

A. hirsuta, Sroz. Hab. : Nexico, Cuba ; Jamaica, common, Port Royal, St. Andrews, at 4,000 feet alt. Grisebach.

A. tomentosa, Swz. Hab. : Mexico; West Indies. 
Anemia-continued.

A. adiantifolia, Swa. Hab. . Mexico, Bahamas, Cuba; Jamaica, on rocks; Dominica, Guadaloupe.

A. cuneata, Kunze. Hab. : Uuba. \$.

A. (Anenidictyon) Phyllitidis, Swz. Hab.: Mexico, Cuba, Jamaica, St. Domingo.

A. (Coptuphyllum) aurita, Swz. Hab.: Jamaica, on calcareous rocks; rare, on the summit of Dolphin's Peak. Grisebach; Hook. Cent. Ferns, t. 3.

A. (Copt.) Wrightii, Baker. Hab. : Cuba. \$.

A. (Copt.) bipinnata, Moore (A. cicutaria, Kze.). Hab. . Bahamas and West Indies.

\section{Iygodium.}

L. digitatum, Eaton. (non Presl.). Hab. : Panama, Guatemala.

L. venustum, Swz. Hab. : Mexico. West Indies: Trinidad, St. Domingo.

L. volubile, Swz. Hab.: Cuba, Jamaica, Trinidad.

L. (Hydroglussum) heterodoxum, Kze. Hab.: Mexico and Guatemala.

\section{Marattia.}

M. (Gymnotbeca) laxa, Kunze. Hab.: Mexico. \$.

M. (Gymno.) alata, Smith. Hab.: Mexico, Guatemala. West Indies: Jamaica, in woods at the base of the Blue Mountains; St. Vincent. Grisebach.

M. (Eupodium) Kaulfussii, J. Sm. Hab. : West Indies.

\section{Dancea.}

D. alata, Smith. Hab.: West Indies: Jamaica, St. Vincent, Cuba, Dominica, Martinique.

D. stenophylla, Kze. Hub. : Guadaloupe; Jamaica, in moist woods. Girisebach. \$s.

D. elliptica, Sm. Hab. : West Indies: Jamaica, in moist woods : St. Vincen:, Trinidad, Guadiloupe. Grisebach.

D. nodosa, Sin. Hab. . Mexico, Cuba, St. Vincent, St. Domingo, Martiaique; Guadaloupe, in woods by streams. Fếe.

\section{Ophioglossum.}

O. nudicaule, L. fll. Hab.: St. Domingo.

o. reticulatum, Linn. Hab.: Cuba; Jamaica, enmmon in coffee plantations; Port Royal, Montserrat, Martinique, Guadaloupe.

O. (Cheiroglossa) palmatum, Linn. Hab. : Cuba, St. Domingo, Guadaloupe.

\section{Botrychium.}

B. virginianum, Swz. Hab. St. Domingo. Fée.

2. VENEZUELA, NEW GRANADA, EQUADOR, PERU, BOLIVIA, GALAPAGOS.

\section{Glelchenia.}

G. longissima, Bl. (G. Bancroftii, Hook.). Hab.: Venezuela, Caraccas, New Grunada.

G. revoluta, $H . \mathcal{B} . K$. Hab.: Andes of Quito; cold and elevated siluations on the Paramo of Saraguru, and on the mountain 
Gleichenia-continued.

called Pulla; at the roots of the Escallonia myrtilloides, at the height of 9-10,000 feet above the sea.-Humb̆oldt. Pichincha, Surucuchu, Peru.

G. simplex, Hook. Hab. : Andes of Quito, Cordillera of Peru ; New Granada. \$.

G. pubescens, $\boldsymbol{H} . \boldsymbol{B} . \boldsymbol{K}$. (Mertensia immersa, $K \boldsymbol{K}$.; Hook. et Grev. Ic. Fil. t. 15). Hab. : Tropical America, abundint. Peru, Tarapota; Equador, Chimborazo, New Granada, Venezuela.

G. glaucescens, Willd. (Mertensia pectinata, Presl.). Hab. ,

Venezuela, New Granada, Peru, Quito.

\section{Cyathea.}

C. Imrayana, Hook. Hab. . Caraccas.

C. cuspidata, Kze. Hab. : marshy woods of Maynas, Peru. \$.

C. divergens, Kze. Hab. : mountains of Pampaynco, Peru ; New Granada; Ecuador, alt. 11,000 feet. \$.

C. gracilis, Griseb. Hab. : Antioquia.

C. equestris, Kze. Hab. : mountains near Pampayaco, Cerro de Cristobal, Peru. \$.

C. Mettenii, Karst. Hab.: Andes of Bogota, alt. 14,200 feet; Tarapota, Eastern Peru. \$

C. squamipes, Karst. Hab. : mountain of Merida, Columbia, alt. 6-9,000 feet; Tarapota, E. Peru. \$.

C. incana, Karst. Hab. : Andes of Bogota, alt. 15,000 feet. $\$$.

C. frondosa, Karst. Hab. : Andes of Bogota, alt. 14,400 feet. \$.

C. Schanschin, Mart. Hab. : Peru.

C. Sprucei, Hook. Hab.: Ecuador, Montaña di Canelos, and Tunguragua, alt. 4-6,500 feet, and Chimborazo, alt. 3-4,000 feet. \$.

C. ebenina, Karst. Hab. : Columbia, alt. 15,000 feet. $\$$.

C. microphylla, Mett. Hab.: Andes of Peru and Ecuador. $\$$. Hook. Second Cent. Ferns, t. 99.

\section{Femitelia.}

H. Karsteniana, $K l$. Hab. : Caraccas. $\$$.

H. grandifolia, Spr. (H. obtusa, Kaulf.). Hab.: N. Granada.

H. subincisa, Kze. Hab. . Tropical Ámerica (Pacific side), Peru, Andes of Ecuador. zuela.

H. horrida, Br. Hab. : New Granada, Ecuador, Peru, Vene-

H. speciosa, Hook. Hab.: New Granada, Caripe, Caraccas. \$. H. Lindeni, Hook. Hab. : Caraccas. $\$$.

H. bella, Reichenb. f. Hab. : Cartuccas. \$.

H. Guianensis, Hook. Hab.: Caraccas, Eastern Peru, and Ecuador.

\section{Alsophila.}

A. blechnoides, Hook. Hab. : Veneznela, Peru.

A. phegopteroides, Honk. Hab. . Tarapota, E. Peru. \$.

A. paleolata, Mart. Hab.: Peru.

A. procera, Klfs. (A. Hookeriana, Klotzsch). Hab.: Venezuels, East Peru.

A. armata, Pr. Hab.: New Granada.

A. Gardneri, Hook. Hab.: Caraccas. 
Alsophila-continued.

A. aspera, Br. Hab. : New Granada, S. Darien.

A. Leucolepis, Mart. Hab.: Venezuela.

A. infesta, Kze. (A. phalerata, Mart.). Hab.: Venezuela, Now Granada; Peru.

A. atrovirens, $\operatorname{Pr}$. (A. compta, Mart.). Hab. : Caraccas.

A. elongata, Hook. Hab. : New Granada, Isle of Tumaco, Esmeraldas.

A. Poeppigii, Hook. Hab. : New Granada, Peru. \$.

A. villosa, Pr. (A. Humboldtii, $K l$.). Hab. : Venezuela, New Granada; near Caripe, in New Andalusia, and the table land of Guardia de San Augustin, and the village of Santa Cruz, Caraccas ; well figured by $\boldsymbol{H} . \boldsymbol{B}$. $K$. and by Martius.

A. pycnocarpa, Kze. Hab. : woods of Pampay, Peru. \$.

A. microphylla, $K l$. Hab. : Caraccas. \$.

A. ? sagittifolia, Hook. Hab.: Trinidad? (possibly the opposite coast of Venezuela). $\$$.

feet.

A. melanopus, Hook. Hab.: in woods, Chimborazo, alt. 3,000

A. Chimborazensis, Hook. Hab.: Chimborazo, alt. 3-4,000 feet. $\$$.

A. conjugata, Spruce. Hab.: Chimborazo, alt. 3-4,000 feet. \$.

A. Sprucei, Hook. Hab.: Chimborazo, alt. 2,500 feet. $\$$.

A. aterrima, Hook. Hab.: Tarapota, E. Peru. \$.

A. frigida, Karst. Hab. : Andes of Bogota, alt. 15,300 feet. \$.

woodsia.

W. mollis, J. Sm. Hab.: Andes of Peru and Quito.

W. Yeruviana, Hook. Hab. : shady places, Andes of Peru. \$.

\section{Dicksonia.}

D. Sellowiana, Honk. Hab.: Peru.

D. coniffolia, Hook. Hab. : Caraccas and New Granada.

D. Plumieri, Hook. Hab. . Caraccas.

D. adiantoides, $H . B . K$. Hab. : Caraccas, Venezuela, New Grazada, Quito.

Peru.

D. cicutaria, Swz. Hab. : Guayaquil ; Quebrada of Panahuanca,

D. apiifolia, Szz. Hab.: Andes of Ecuador and Peru. \$.

D. rubiginosa, Kaulf. Hab. : New Granada, Ecuador, Tarapota, Peru, Venezurla.

D. obtusifolia, Willd. (D. Pavoni, Hook.). Hab. . Andes of Peru. \$.

\section{Deparia.}

D. concinna (D. Matthewsii, Hook.). Hab. : Tarapota.

\section{Eymenophyllum.}

H. abruptum, Hook. Hab.: Venezuela.

H. ‘xillare, Suz. Hab.: Venezuela.

H. renitorme, Hook. Hab. : Andes of Ecuador and Peru. \$.

H. crispum, $\boldsymbol{H}$. $\boldsymbol{B} . \boldsymbol{K}$. Hab. : declivities of Munnt Silla de Caraccas, elevation 6.000 feet; Venezuela.

H. undulatum, Sar. Hab. : muuntains of Andinarca, Peru.

H. polyanthos, Swz. Hab.: widely diffused throughout the 
Hymenophyllum-continued.

tropics, and extending a little beyond them north and south; Chacapoyas, Peru.

H. Andinum, $V . D . B$. Hab. : Andes of Ecuador. \$.

H. caudiculatum, Mart. Hab. . Peru. $\$$.

H. Lindeni, Hook. Hab.: Andes of Venezuela and Ecuador.

H. microcarpum, Desv. Hab. : trunks of trees, Peru.

H. valvatum, Hook. et Grev. Hab. : Columbia, trunks of trees; forest of Esmeraldas, at an elevation of $3-4,000$ feet, and on the descent from Mollituro to Naransal, elevation of 6,000 feet. Hook. \& Gr. Ic. Fil. t. 219.

H. Sprucei, Baker. Hab. : Tarapota, E. Peru. \$\$\$

H. interruptum, Kunze. Hab.: woods, Pampayaco, pendulous from the trees; descent from Mollituro to Naransal, 6-9,000 feet.

H. lineare, Swz. (H. elegans, Spr.). Hab. : Andes of Ecuador and Peru. (H. trifidum, Hook. \& Grev. Ic. Fil. t. 196.)

H. elegantulum, $I, D . B$. Hab. : Andes of Ecuador and Columbia, ascending to 13,000 feet. $\$$.

H. Pistuensis, Hook. MSS. Hab.: Volcano of Pasto, Andes of N. Ecuador. \$.

H. Tunbridgense, Smith. Hab.: Venezuela and Peruvian Andes.

H. tenerrinum, F. B. D. Hab. : Peru. $\$$.

H. Jamesoni, Hook. Hab. : Andes of Columbia. \$.

H. fucoides, Suz. (H. cristatum, Hook. \& Gr. t. 148; H. Peruvianum, Hook. \& Gr. t. 208). Hab.: Andes of Quito, on the trunks of trees near the limits of perpetual snow, at 14,000 feet of elevation above the sea; Peru, Caraccas.

\section{Trichomanes.}

T. elegans, Rudge. Hab.: Peru.

T. spicatum, Hedw. Hab.: Ecuador. Hook. Gard. Ferns, t. 60.

T. Krausii, Hook. \& Gr. Hab.: Andes of Ecuador and Peru.

T. Bancroftii, Hook. \& Gr. Hab. : Peru. Hook. \& Gr. Ic. Fil. t. 204 .

T. alatum, Suz. (T. attenuatum, Hook.). Hab.: Venezuela.

T. brachypus, Kunze (T. Ankersii, Parker; Hook. \& Gr. Ic. Fil. t. 201). Hab. : Peru.

T. radicans, Sioz. (Hrmenophyllum rupestre, Raddi). Hab.: forest of Fsmeraldas, El Equador.

T. pyxidiferum, $L$. Hab. . Peru.

T. crispum, L. Hab. : Peru.

T. crinitum, $L$. Hab. : Andes of Ecuador.

T. lucens, Swz. Hab. . Peru.

T. Lambertianum, Hook. Hab. : woods at Pillao, Peru. \$.

T. pinnatum, Swz. (T. floribundum, H. B. K.). Hab. : Peru. Hook. \& Gr. Ic. Fil. t. 9.

T. tentrum, Sprengel (T. angustatum, Carm.). Hab. : Esmeraldas, Ecuador and Pichincha, Peru. Hook. \& Gr. Ic. Fil. t. 166.

T. trichoideum, Swz. Hab : between Quito and Esmeraldas, growing abundantly on the trunk of a tree at an elevatinn of 6,000 feet above the level of the sea. Hook. \& Gr. Ic. Fil. t. 199. 
Trichomanes-continued.

T. rigidum, Swz. Hab. : Peru.

\section{Davallia.}

D. inæqualis, Kunze. Hab. : Peru.

1. concinna, Schrad. (D. Schimperi, Hook.; D. Lindeni, Hook. ;

D. thecifera, $H . B . K$.). Hab. . Andes of Columbia, Caraccas.

\section{Cystopteris.}

C. fragilis, Bernh. Hab. : Andes of Peru ; Quito.

\section{Iindsaya.}

L. trapeziformis, Dry. (L. quadrangularis, Raddi ; L. horizontalis, Hook. ; L. arcuata, Kze. ; L. caudata, Hook. ; L. Leprieurii, Hook.). Hab. : woods, Pampayaco, Peru; Venezuela, New Granada.

L. Guianensis, Dry. Hab. : Venezuela.

L. stricta, Iry. Hab. . throughout tropical America, especially on the western side; Columbia.

L. Michleriana, Eaton. Hab.: Cataract of Truando, New Granada. $\$$.

\section{Dictyoxiphium.}

1. Panamense, Hook. Hab. : New Granada.

\section{Adiantum.}

A. lunulatum, Burm. (A. dolabriforme, Hook.; A. deflectens, Mart.). Hab.: Venezuela.

A. Kaulfussii, Kunze. Hab.: New Granada; Venezuela ; Chatham Island, near Galapagos.

A. obliquum, li illd. Hab. : Caraccas, Columbia.

A. Peruvianum, Klotzsch. Hab. : Peru. \$\$.

A. internedium, Swartz (A. foveanum, Raddi; A. triangulatum, Kaulf.). Hab.: Guayaquil, Peru ; Esmeraldas.

A. sinuosum, Gard. Hab.: Cerro of Santana, Guayaquil; El Equador.

A. trapeziforme, L. (A. pentadactylon, Langs, et Fisch.). Hab. : Caraccas.

A. polyphyllum, Willd. (A. cardiochlæna, Kunze; A. Matthewsianum, Hook.; A. macrocladum, Klotzsch). Hab. : Columbia and Chacapoyas, Peru; Caraccas, Cumanacoa, New Andalusia. Humboldt. $\$$.

A. glaucescens, Klotzsch. Hab.: Ecuador.

A. Henslovianum, Hook. fil. (A. sessilifolium, Hook.). Hab. : Columbia, Chacapoyas. Peru; James and Charles Islands, Galapagos Group. C'. Darwin. \$\$.

A. obtusum, Desv. Hab.: Myobamba, Peru, New Granada. Hook. \& Gir. Ic. Fil. t. 188.

A. cristatum, L. (A. Kunzeanum, Klotzsch). Hab.: Venezuela, Caraccas.

A. pectinatum, Kunze. Hab. : Eastern Peru.

A. tetraphyllum, Willd. (A. prionophyllum, H. B. $K$. ; A. fructuosum, Spr.). Hab. : Esmeraldas, l'umaco.

A. Puyllitidis, J. Smith. Hab.: Eistern Peru.

A. macrophyllum, Swz. Hab.: New Granada, Venezuela, Caripe, New Andalusia. Hook. \& Gr. Ic. Fil. t. I32.

A. villosum, $L$. (A. falcatum, Suz.). Hab.: New Granada. 
Adiantum-continued.

A. pulverulentuin, $L$. Hab. : New Granada.

A. incisum, Presl. Hab.: Columbia, Galapagos Islands.

A. Capillus-veneris, $L$. Hab. : Venezuela.

A. Athiopicum, L. Hab.: Caraccas, Quito, Peru, Mendoza.

A. concinnum, $H . B . K$. Hab. : Quebrada del Cuchivano, near Cumanacoa; Guayaquil, Chacapoyas, Peru; Galapagos Group, Andes of Quito.

A. colpodes, Moore. Hab.: Ecuador and Peru.

A. dipitatum, Presl. (A. speciosum, Hook.). Hab. : Peru ; Bolivia, about the village of Sasaranga, El Equador.

A. patens, Willd. Hab.: Galapages Isles.

A. dolosum, Kunze. Hab.: Granada.

\section{Irypolepis.}

H. repens, Presl. Hab.: Galapagos, Peru, Columbia.

H. hostilis, Presl. Hab.: Hualaga, Upper Peru.

H. Purdieana, Hook. Hab.: Paramo of Ruiz, N. Granada. \$.

H. parallelogramma, Hook. Hab.: woody mountains of Pampayaco; Peru, bamboo-grounds, rare; Tovar, Columbia.

\section{Chellanthes.}

Ch. radiata, B. Br. (Hypolepis radiata, Hook.). Hab.: Peru, Columbia.

Ch. dichotoma, Swz. Hab. : Mount St. Antonio, Quito.

Ch. micropteris, Swoz. Hab.: Ecuador, Peru.

Ch. Matthewsii, Kunze. Hab.: Andes of Bolivia and Peru; crevices of rocks and dry places, Huamantaga.

Peru. \$.

Ch. pilosa, Goldm. (Ch. MacLeanii, Hook.). Hab.: Andes of

Ch. microphylla, Desv. Hab. : Andes of Quito, Loja, Equador ; San Rafael, Huanuco, elevation 6,000 fert; Peru, Chacapoyas and Purnchuca, on dry sandy banks; Bolivia.

Ch. viscosa, Kaulf. Hab.: Venezuela.

Ch siariosa, Kaulf. Hab.: Andes of Peru, fissures of rocks between San Rafael and Huanuco; limestone rocks, ascending the Cordillera from Tarma. \$\$.

Ch lendigera. Suz. Hab.: Andes of New Granada and Equador. Ch. farinosa, Kaulf. Hab. : New Granada.

\section{Pollaea.}

P. geraniæfolia, Fée. Hab. : Santa Marta, Columbia, Galapagos.

P. rigida, Hook. Hab.: Peru.

P. (Allosorus) ambigua, Baker (Cheil., Mett.). Hab. : New Granada. $\$$.

P. (Allo.) ternifolia, Fée. Hab. : Andes of Peru; Quito, on old walls.

P. (Allo.) pulchella, Fée. Hab.: lofty Andes of Peru.

P. (Allo.) maruinata, Baker (Cheilanthes, $\boldsymbol{H}$. B. $\boldsymbol{K}$.). Hab. : rocky places, Peripe; Andes of Quito, 7-8,000 feet elevation, and on old walls near Quito and Peru.

P. (Platyloma) cordata, J. Smith. Hab.: Columbia and Andes of Peru.

Pteris.

Pt. longifolia, L. Hab.: Venezuela. 
Pteris-continued.

Pt. quadriaurita, Retz. Hab.: Quebrada of Huanacabra, Peru; New Granada, Venezuela.

Pt. coriacea, Desv. (Pt. Jamesoni, Hook.). Hab. : Andes of Quito and Peru.

Pt. aquilina, L. Hab.: Galapagos, Peru.

Pt. viscosa, Baker (Pt. scalcris, Moritz.). Hab. : Peru.

Pt. (Doryopteris) palmata, Willd. (Pt. pedata, Hook. Sp. Fil.). Hab.: Culumbia, southward to Peru; Galapagos. Hook. Gard. Ferns, t. 22.

Pt. (Litobrochia) Henkeana, Presl. Hab. : Peru, Pampayaco, Ocaña, New Granada.

Pt. (Lito.) vestita, Baker. Hab. : Peru. \$.

Pt. (Lito.) aculeata, Swz. Hlab.: New Granada; Ocaña, 5,000 feet elevation; Ecuador, Venezuela.

Pt. (Lito.) incisa, Thunbg. Hab. : Columbia, Venezuela.

Pt. (Lito.) podophylla, Swz, Hab.: Venezuela, Caraccas, Bogota; Ocaña, alt. 4-5, (00 feet. Hoo\%. Gard. Ferns, t. 55.

Pt. (Lito.) Kunzeana, Agardh. Hab.: Ecuador, Peru.

Pt. (Lito.) elata, Ayardh. Hab. : Ecuador.

Pt. (Lito.) gigantea, Willd. (Pt. crassipes, Ag.). Hab. : Columbia, southward to Peru.

Pt. (Amphiblestra) latifolia, H. B. $\boldsymbol{K}$. Hab.: Venezuela; Quebrado del Cuchirano, near Cumançoa, and mountains of Caripe, elevation 3,000 feet. $\$$.

\section{Iomaria.}

L. attenuata, Willd. Hab.: Columbia.

L. L'Herminieri, Bory. Hab. . Columbia, southward to Chili; Caraccas, Tovar, Santa Mlarta. Peru.

L. divergens, Kunze (L. Plumieri, Desv.). Hab. : Columbia, feet.

L. onocleoides, Spreng. Hab. : Pasto; Ecuador, elevation 10,000

L. procern, Spreng. (L. Gilliesii, Hook. \& Gr. Ic. Fil. t. 207). Hab.: Venezuela, Caraccas, Peru, Hook. Gart. Ferns, t. 63.

L. caudata, Baker. Hab.: Andes of Ecuador. \$.

L. Boryana, Willd. (L. Magellanica, Desv.). Hab.: New Granada, Ocrũa, Merida, Venezuela, Peru. Hook. Gard. Ferns, t. 52.

L. acuta, Desv. (L. cuspidata, Kze.). Hab.: New Granada, Pampayaco ; Peru, parasitic nn Cyathaacea; on Mount Campana, near 'Tarapota, Eastern Peru. \$.

L. semicordata, Baker (L. biserrata, M. \& L.). Hab. : Pasto, Peru, Columbia, Torar, Merida, Ucaña.

\section{Blechnum.}

B. I,anceola, Swz. Hab. : Tarapota, Eastern Peru, on the Campana Mountrin ; Boqueta, Veraguas.

B. asplenioides, Swz. Hab. . Pert, New Granada.

B. unilatarale, Willd. (B. polypodioides, Raddi). Hab. : Tarapota, E. Peru; Venezuela, New Granada.

B. Brasiliense, Desv. (B. Corcovadense, Raddi). Hab.: Tarapota. Eastern Peru.

B. Iongifulium, $\boldsymbol{H} . \boldsymbol{B} . \boldsymbol{K}$. Hab. : Tarapota; Venezuela, abundant. New Granada, St. Marta, Sierra Nevada, Cumana, Ocaña. 
Blechnum-continued.

B. Fendleri, Hook. Hab. : Tovar, Venezuela.

B. occidentale, Linn. Hab. : Tarapota, Galapagos, Andes of Quito, Venezuela.

B. serrulatum, Rich. Hab. : Guayaquil.

B. volubile, Kaulf. Hab. . Tarapota, E. Peru ; Columbia.

\section{Asplenium.}

A. serratum, Linn. Hab. : Peru, Galapagos Isles.

A. subhastatum, Hook. Hab. : Peru, Caraccas. \$. Hook. Cent. Ferns, 29.

A. projectum, Kunze. Hab. : warm rocks, Pampayaco; Peru. \$.

A. fragile, Presl. Hab.: Andes of Peru; Chimborazo, on rocks at an elevation of 14,000 feet; Columhia. Hook. Cent. Ferns, t. 32 .

A. Gilliesianum, Hook. Hab.: Andes of Peru and Bolivia; fissures of rocks at Ceno Grande, Uspallata, Andes of Mendoza. \$.

A. Quitense, Hook. Hab. : Andes of Ecuador, on decayed trees; Forest of Archeduna. Andes of Quito. $\$$.

A. Trichomanes, L. Hab.: Andes of Peru, mountains on the river Mayo, near Tarapota; Quito, Caraccas, Merida.

A. extensum, Fée. Hib.: New Granada; moist grottoes in forests of Ocaña, elevation 7,000 feet; Andes of Peru. $\$$.

A. monanthenum, Linn. Hab. . Columbia, Bogota, Andes of Quito and Peru.

A. ebeneum, Ait. Hab.: Valley of Chillo, on the walls of the Haçienda de Arcadia, near Quito; New Granada, Rio Hacha, Sierra Nevada, 10,000 fert elevation.

A. alatum, H. B. K. Hab.: New Granada, Venezuela; Forest of Archedona, Ecuador, Peru; Tarapota.

A. salicifolium, Linn. Hab. : New Granada, Venezuela, Peru.

A. virens, Prest. Hab.: Gorgona Island and Guayaquil.

A. oligophyllum, Kaulf. Hab. . Venezuela, Ocaña, New Granada.

A. erectum, Bory. Hab. . Venezuela, New Granada, Ecuador, Esmeraldas, Pichincha, Tarapota, E. Peru.

A. obtusifolium, L. (A. jiparium, Liebm.). Hab. : moist places, and even in water; New Granada, Venezuela; western declivity of Pichincha.

A. firmum, Kunze. Hab.: Merida, Venezuela; Columbia, Peru.

A. auriculatum, Swz. Hab.: Peru, Tarapota, Archedona, Quito.

A. anisophyllum, Kunze. Hab. : Galapagos.

A. rhiznphorum, Linn. Hab.: Venezuela, Tovar, Caraccas, N. Granada, Peru, Tarapota.

A. hastatum, Klotzsch. Hab.: Ecuador, New Granada, Venezuela, Tovar; woods of Guachapala, and Andes of Quito, elevation 6,000 feet. \$.

A. vomeriforme, Hook. Hab.: Peru. \$.

A. Serra, Langs. \& Fisch. Hab.: Venezuela, Ocaña, Caraccas, Ecuador ; Peru.

A. auritum, Sivz. Hab.: Ecuador, Andes of Quito, 6,000 feet elevation; Chatham Island, Galapagos, New Granada.

A. dimidiatum, Swz. Hab.: Venezuela, N. Granada; Ocaña, 7,000 feet; Peru, province of Chacapoyas. 
Asplenium-continued.

A. formosum, Willd. Hab. : Chatham Island, Galapagos; Peru.

A. pulchellum, Raddi. Hab.: Peru, high mountains, Sierra Nevada de Santa Marta.

A. lætum, Swartz. Hab.: Venezuela; Chatham Island, Galapagos.

A. bissectum, Swz. Hab. : Ecuador.

A. Hallii, Hook. Hab. : Forest of Esmeraldas, Ecuador, Sao Gabriel; valley of the Amazon, on young trees and shrubs. Hook. Second Cent. Ferns, t. 30.

A. furcatum, Thunb. Hab.: Ecuador, walls of Quito; Peru, Isle of Gorgona, Bogotá, Galapagos.

A. scandicinum, Kaulf. Hab.: Ecuador.

A. fragrans, Szoz. Hab.: New Granada, Ecuador; Andes of Quito, nerr Papallacla, New Andalusia, Peru, Tarapota.

A. psendo-nitidum, Raddi. Hab. . Venezuela, Ecuador.

A. Jamesoni, Hook. Hab.: Guayaquil, Andes of Ecuador. \$.

A. equamosum, Linn. Hab.: Venezuela, Tovar, Ecuador, Cordillera of Bogotá.

A. tripbyllum, Presl. Hab. : Cordillera of Peru, Cerro Pasco and Huamantanga; Valley of Canta, Quitinian Andes, elevation 15,000 feet; Chimborazo and Antisana, Ecuador, 13,000 and 14,000 feet elevation. $\$$.

A. repens, Hook. Hab.: Andes of Ecuador, growing on trees and shrubs in the forests of Archedona, near Quito. Hook. Second Cent. Ferns, t. 31. \$.

A. delicatulum, Presl. Hab.: Andes of Quito, on trunks of trees, Cuchaos; Venezuela; N. Granada. Hook. Cent. Forns, t. 18.

A. divaricatum, Kunze. Hab.: Andes of Peru.

A. rhizophyllım, Kunze. Hab.: N. Granada, Sierra Nevada, Venezuela, Tarapota, E. Peru ; Andes of Quito, forest of Archedona, Galapagos Isles.

A. rutacenm, Mett. Hab.: N. Granada, Venezuela, Tovar, Ocaña and Sierra Nevada, elevation 6,000 feet; Equador; Andes of Quito, in trunks of trees, forests of Archedona; Galapagos Islands. $\$$.

A. cicutarium, Saz Hab.: Ecuador; Tarapota, Forest of Archedona, near Quito, Galapagos.

A. (Darea) ferulaceum, Moore. Hab. : Now Granada, Andes of Ecuador, Quito. \$\$.

A. (Athyr.) Filix-foemina, Bernh. Hab. . Caraccas and Venezuela.

A. (A thyr.) aspidioides, Mett. Hab.: Ecuador.

A. (Diplazium) plantagineum, $L$. Hab.: Tovar, in Venezuela ; New Granada, Ocaña, elevation, 5,000 feet.

A. (Dipl.) Lechleri, Mett. Hab.: Peru, Mount Guayrapurima, near Tarapota. $\$$.

A. (Dipl.) Callipteris, Baker. Hab.: Tarapota.

A. (Dipl.) grandifolium, Swz. Hab.: Ocaña, elevation 7,000 feet; woods of the Andes of Quito and Bay of Utria, west coast of Darien.

A. (Dipl.) flavescens, Mett. (A. juglandifolium, Lam.). Hab. : Veneztula, Peru.

A. (Dipl.) rhoifolium, Mett. Hab. : N. Granada. \$. 
Asplenium-continued.

A. (Dipl.) celtidifolium, Kunze. Hab.: Venezuela, N. Granads, Ocaña, Ecuador.

A. (Dipl.) arboreum, Willd. (D. auricnlatum, Kanlf.). Hab. : N. Granada, Ocaña, elevation 8-10,000 feet; Caraccas, Venezuela, near Caripe.

A. (Dipl.) Shepherdi, Spreng. (A. striatum, Linn.). Hab. . Venezuela, Tocar, Tarapota, Peru.

A. (Dipl.) Sprucei, Baker. Hab. : Andes of Fcuador. \$.

A. (Dipl.) crenulatum, Baker. Hab.: Ecuador.

A. (Dipl.) costale, Swz. Hab.: N. Granada, Ecuador, Forest of Archedona, Andes of Quito, Tarapota, St. Gavan, Peru.

A. (Jipl.) Franconis, Mett. Hab.: Ecuador.

A. (Dipl.) nervosum, Mett. Hab.: N. Gianada. \$".

A. (Dipl.) venulosum, Baker. Hab.: Andes of Ecuador. \$.

A. (Dipl.) pulicosum, Hook. Hab. : Ecuador. \$.

A. (Dipl.) Klotzschii, Mett. Hab. : Columbia and Venezuela. \$.

A. (Dipl.) Hexuosum, Presl. Hab. : valleys of the Cordillera of

Peru. \$.

A. (Dipl.) vastum, Mett. Hab. : New Granada. $\$$.

A. (Dipl.) hians, Kunze. Hab.: New Granada, Merida, Ecuador.

A. (Dipl.) radicans, Schk. Hab.: New Granada, Caraccas; Tarapota, E. Peru.

A. (Dipl.) gracilescens, Mett. Hab. : Venezuela. $\$$.

A. (Dipl.) Sandwichianum, Mett. (A. fisco-pubescens, Hook.). Hab.: New Granada, Ocaña, in forests, 4-5,000 feet elevation; Mount Campaña, near Tarapota, Perı.

A. (Dipl.) divisissimum, Baker. Hab. : foot of Mount Chimborazo. \$.

A. (Anisogonium) Kunzei, Mett. Hab.: Pampayaco, Peru ; Mount Guayrapurima, near Tarnpota, Archedona, Ecuador. $\$$.

A. (Aniso.) Chimborazense, Sipruce MSS. Hab. . foot of Chimborazo, elevation 3-4,000 feet. $\$$.

A. (Aniso.) riva]e, Spruce MSS. Hab. : foot of Chimborazo, elevation $3-4,000$ feet. $\$$.

A. (A niso.) stenocarpum, Mett. Hab. : N. Granada. \$.

A. (Hemidictrium) Purdieanum, Hook. Hab.: Peru, Venezuela. Hook Cent. Ferns, t. 37.

A. (Hemi.) marginatum, Linn. Hah.: Tarapota, E. Peru ; Venezuela. One of the finest and most common of tropical American Ferns, on the borders of streams, and in damp, moist, shady woods.

Didymochizena. Peru.

D. lunulata, Desv. Hab. : New Granada, Andes of Ecuador and

\section{Aspidium.}

A. (Polystichum) semicordatum, Suz. Hab.: New Granada, Venezuela, Esmeraldas, Ecuador, Tarapnta, Galapagos Islands.

A. (Polyst.) aculeatum, Suz. Hab.: Peru, Tarapota, Chacapoyas, Eeuador, Quito, Tunguraqua.

A. (Cyrtomium) abbreviatum, Schrad. Hab. : Pernambuco, N. Granada; Rio Hacha, elevation 9,000 feet. 
Aspidium-continued.

A. plantagiveum, Griseb. Hab.: Tarapota, E. Peru, Venezuela, mossy rocky places near water.

Peru.

\section{mephrodium.}

N. (Lastrea) Braunianum, Hook. Hab. : on trees and stones in shady woods of the eastern Cordillera of Bogotá, near Villavicenzio. $\$$.

N. (I,ast.) pusillum, Baker. Hab. : New Granada. \$.

N. (Last.) semihastatum, Hook. Hab.: Peru, dense woods at Pampayaco. \$.

N. (Last.) macrotis, Hook. Hab. : Tarapota, E. Peru. \$.

N. (Last.) insigne, Baker. Hab. : New Grannda. \$.

N. (Last.) chrysolobum, Fée. Hab.: Columbia.

N. (Last.) macrourum, Baker (N. schizotis, Hook.). Hab. . Caraccas, near Tarapota, Eastern Peru.

N. (Iast.) patens, Desv. Hab. : N. Granada, Ecuador, Peru.

N. (Last.) abruptum, Baker. Hab. . Peru. \$.

N. (Last.) invisum, Baker (N. macrourım, Hook.; N. Serra,

Desv.). Hab. : Venezuela, N. Granada, Bogotá; Ecuador, Chimborazo, elevation 4,000 feet; Peru.

N. (Last.) falciculatum, Desv. Hab. : Peru.

N. (Last.) Caripense, Houk. Hab. : Caripe, Venezuela. Tovar.

N. (Last.) triste, Hook. Hab. : N. Granada, Andes of Ecuador, Tarapota, E. Peru.

N. (Last.) tetragonum, Hook. Hab. . N. Granada, Peru.

N. (Last.) Leprieurii, Hook. Hab.: Andes of NE. Peru.

N. (Last.) conterminum, Desv. Hab.: Venezuela, Ecuador, Tunguragua, Tarapota.

N. (Last.) Sprengelii, Hook. Hab.: Columbia.

N. (Last.) resino-foetidum, Hook. Hab. : Andes of Ecuador, foot of Chimborazo, altitude 3-4,000 feet, in moist woods. 8 .

N. (Last.) Sprucei, Baker. Hab. : Andes of Licuador. \$.

N. (Last.) diplnzioides, Hook. Hab. : Columbia, Tovar. $\$$.

N. (Last.) microsorum, Hook. Hab. : font of Chimborazo. \$.

N. (Last.) Filix-mas, Rich. Hab.: Ecuador and Peru.

N. (Last.) mexicanum, Hook. Hab. : Andes of Ecuador, Venezuela, N. Granada.

N. (I Last.) catocarpum, Hook. Hab.: Venezuela. \$.

N. (I ast.) furcatum, Hook. Hab. : Columbia, Tarapota, E. Peru, foot of Chimborazo, alt. 3,000 feet.

N. (Last.) villosum, Presl. Hab. : N. Granada, Ecuador, foot of Chimborazo.

N. (Last) Trianz, Baker. Hab. . N. Granada. \$.

N. (Last.) acutum, Hook. Hab. : Trapota, E. Peru.

N. (Last.) denticulatum, Hook. Hab.: N. Granada. (Aspid. Klotzschii. Hook. Second Cent. Ferns, t. 23.)

N. (Last.) effusum, Baker (N. amplissimum, Hook.). Hab. : Caraccas, Venezuela, Peru.

N. Skinneri, Hook. Hab. : Bombinasa, Andes of Ecuador.

N. incisum, Baker (N. Stenopteris, Hook.). Hab. : N. Granada.

N. unitum, $R$. Br. (N. propinquum, Br.). Hab. : Peru. 
Nephrodium-continued.

N. molle, Desv. Hab. : Ecuador, Esmeraldas, Tarapota; N. Granada, Venezuela.

N. brachyndon, Hook. Hab.: Pampayaco, Peru; coast of Ecuador; frot of Chimborazo; Galapagos.

N. Fendleri, Honk. Hab. : Torar, Venezuela.

N. (Sagrenia) subtriphyllum, Baker. Hab. : coast of Ecuador.

N. (Sagen.) latifolium, Baker. Hab : Ecuador.

N. (Sagen) elatum, Bakrr. Hab.: foot of Chimborazo. \$.

N. (Saren.) cicutarium, Baker. Hab.: foot of Chimborazo, elevation 3,000 fret.

N. (Sagen.) macrophyllum, Baker (Aspid., Sw.). Hab.: N. Granada, Caraccas; Ecuador, foot of Chimborazo, 3,000 fret elevation; Esmeraldas, Quitian Andes, banks of the Napo; Tarapota, Galapagos.

\section{wephrolepis.}

N. cordifolia, Baker (N. tuberosa, Pr.). Hab.: N. Granada, Ecuadnr. Pichincha and Ficuador.

N. exaltata, Schott. Hab. : Peru, Ecuador, foot of Chimborazo; pendulous from the branches of trees, sterile fronds sometimes twelve feet long.

N. acuta, Presl. Hab. : Peru.

\section{Oleandra.}

O. neriiformis, Cav. Hab.: N. Granada, Peru.

\section{Polypodium.}

P. (Pheg.) flavo-punctatum, Kaulf. Hab. . Caraccas, Venezuela, Ocaña, Tarapota, E. Peru.

P. (Pheg.) alleopterum, Kunze. Hab.: Columbia. \$\$.

P. (Pbeg.) Tijuccanum, Raddi. Hab. . San Gabriel and Tarapota, Eastern Peru.

P. (Pher.) deflexum, Baker. Hab.: N. Granada. \$.

P. (Pheg.) refulgens, Klotzsch. Hab. . Columbia.

P. (Pheg.) rude, Kze. Hab.: N. Granada, Venezuela, Ecuador, Peru, Galapagos.

P. (Pher.) decussatum, $L$. Hab.: Venezuela, Ecuador, Peru.

P. (Pheg.) platylobun, Baker (P. rotundatum, Hook.). Hab.: Tarapota, E. Perlu. \$s.

P. (Pheg.) fibrillosum, Baker. Hab.: Antes of Peru. \$.

P. (Pheg.) macrophyllum, Hook. Hab.: Tarapota. \$.

P. (Pheg.) pteroideum, Klotzsch. Hab.: Ecuador, N. Granada. \$s.

P. (Pheg.) biseriale, Baker. Hab. : Andes of Peru and Ecuador. \$.

P. (Pher.) platyphvllum, Hook. Hah.: Caraccas, Venezuela, Andes of Er-uador; Chimbornzo, alt. 3,000 feet; Tunguragua, alt. 7,000 feet; Tarapota.

P. (Pheg.) rigidum, Hook. \& Grev. Hab.: Andes of Peru and Ecnador, alt. 1z-16,000 feet.

P. (Pheg) punctatum, Thunb. Hab.: Ecuador, Pern.

P. (Pheg.) prasinum, Baker (P. punctatum, Spruce). Hab.: Tarapota, E. Peru, and foot of Chimborazo; Ecuador, in woods, alt. 3,000 feet. 
Polypodium-continued.

P. (Cyrtomiphlebium) dulium, Hook. Hab. : Andes of Ecuador and Peru; Bogota.

P. (Goniopteris) diversifolium, Suz. Hab.: Caraccas, Ecuador.

P. (Goniopt.) crenatum, Swz. Hab. Ecuador, Guayaquil.

P. (Goniopt.) stramineum, Baker (P. salicifolium, Hook.). Hab.: Tovar, Venezuela. \$.

P. (Goniopt.) tetragonum, Swz. Hab.: Peru.

P. (Dictyopteris) draconopterum, Hook. Hab.: N. Granada, Ecuador; Chimborazo, alt. 3,000 feet; Tarapota. \$.

P. Sprucei, Hook. Hab.: near Tarapota. $\$$.

P. jungermannioides, Klotzsch. Hab.: Peru.

P. chrysolepis, Hook. Hab.: Andes of Quito, creeping among mosses. \$ $\$$

P. (Grammitis) marginellum, Swz. Hab.: Venezuela, Tarapota, Eastern Peru.

P. Andinum, Hook. Hab.: Andes of Quito, near the River Hondache, and Cerro de Abitagua, on trees; Mount Picóte, near Mayobamba, Peru. Hook. Second Cent. Ferns, t. $6 . \$$.

P. trichosorum, Hook. Hab.: on trees, Forest of Archedona, Quitinian Andes. $\$$.

P. trifurcatum, $L$. (P. comptoniæfolium, Desv.). Hab. : on trees, Ecuador; Forest of Archedona, and at Abitagua. Hook. \&Gr.Ic. Fit. t. 42. (P. scolopendriodes.)

P. serrulatum, Mett. Hab.: Peru. (Xiphopteris Jamesoni. Hook. Second Cent. Ferns, t. 14.)

P. subdimidiatum, Baker. Hab.: Venezuela, Andes of Ecuador. \$.

P. moniliforme, Lagase. Hab. : Ecuador and Peru, at elevations even of 10,000 feet.

P. trichomanoides, Swz. Hab.: Ecuador.

P. flabelliforme, Lam. Hab. . Columbia, Ecuador. On trees, Ocaña ; Quitinian Andes, Peru. $\$$.

P. subtile, Kunze. Hab.: Venezuela, Andes of Ecuador, Peru.

P. delicatulum, Mart. et Gal. Hab.: Tunguragua; Ecuador, on mossy stones.

P. Peruvianum, Desv. Hab.: Venezuela; Peru, near Pasco, Merida. \$.

P. pilosissimum, Mart. et Gal. Hab. : N. Granada, Surucucho, Ecuador; Ocaña, elevation 8,000 feet. Peru.

P. cultratum, Willd. Hab.: Venezuela, Andes of Ecuador,

P. Adenophorus, Hook. et Arn. Hab.: Peru.

P. decipiens, Hook. Hab.: Columbia, Forest of Archedona; Ecuador, trunks of trees; Sachapata, Peru.

P. subsessile, Baker (P. pteropus, Hook.). Hab.: Venezuela, mossy trunks of trees; Andes of Quito, alt. 3-6,000 feet; and in Mount Abitagua.

P. farinosum, Hook. Hab.: Andes of Ecuador, rare; trunk of an old tree on the eastern descent of the Cordillera of Quito, where the forests commence. Hook. ('ent. Ferns, t. 47. \$.

P. curvatum, Sws. Hab.: New Granada; Ocaña, elevation 
Polypodium-continued.

8,000 feet; Ecuador, mountains near Cuença, elevation 12,000 feet, on rocks; Agapata, Peru.

P. pendulum, Sicz. Hab.: Peru, on the Andes.

P. suspensum, Linn. Hab. : Ecuador; Andes of Quito, alt. 8,000 feet; Columbia, frequent.

P. melanopus, Hook. \& Gr. Hab. : Andes of Ecuador, hanging vertically at Surucucho, near Cuença, from the trunks of trees, elevation 9,000 feet above the level of the sea. $\$ \$$

P. pilipes, Hook. Hab.: Chacapoyas, Province of Myobamba, eastern declivity of the Andes of Peru. \$.

P. macrocarpum, Presl. Hab.: Andes of Peru, Andes above Ticicaca, Bolivia. Hook. Cent. Ferns, t. 34.

P. radicale, Moritz. Hab. : Venezuela.

P. pulchrum, Mart. \& Gal. Hab.: N. Granada.

P. taxifolium, L. (P. Plumula, II. B. K.). Hab.: Peru, Andes of Ecuador.

P. pectinatum, $L$. Hab.: N. Granada, Peru.

P. Meridense, Klotzsch. Hab.: Columbia. \$.

P. Abitaguæ, Hook. Hab.: Andes of Ecuador. \$

P. Moritzianum, Link. Hab. : Venezuela, Tovar. \$.

P. tenuifolium, H. B. K. Hab.: N. Granada, Ecuador.

P. heteromorphum, Hook. et Grev. Hab.: Andes of Ecuador, alt. 12-15,000 feet, growing in large patches over the face of the dripping rocks.

P. semiadnatum, Hook. Hab. : on trumlss of trees near Pasto, Andes of Quito. Hook. Second Cent. Ferns, t. $48 . \$$.

P. dependens, Baker. Hab.: Andes of Ecuador. \$\$.

P. alternifolium, Hook. Hab. : Ecuador, occurring abundantly on the trunks and branches of trees, at elevations between 3,000 and 10,000 feet, near Esmeraldas, and between Cuença and Guayaquil. $\$$.

P. sericeo-lanatum, Hook. Hab.: N. Granada ; Ocaña, alt. 1011,000 feet. Ecuador; Pichincha, alt. 12,000 feet, and woods near Baños, mountains of Guayrapata. \$.

P. firmum, Klotesch. Hab. . N. Granada.

P. lanigerum, Eaton. Hab.: Venezuela. \$.

P. sporadolepis, Kunze. Hab.: N. Granada, Ecuador. \$.

P. plebeium, Sehlecht. Hab. : Antles of Quito, Peru.

P. bianriculatum, Hook. Hab. : Peru.

P. mpriophyllum, Mett. (P. tenuisectum, Hook. in part.). Hab. : trunks of trees near Talanaca, Peru. Hook. Second Cent. Ferns, t. $21 . \$$.

P. achillæfolium, Kaulf. Hab. : Ecuador.

P. longisetosum, Hook. Hab : Andes of Quito. \$.

P. gracile, Hook. Hab.: Iluallnay, near Pasco; Andes of Peru. Hook. \& Gr. Ic. Fil. t. 22.2. \&.

P. athyrioides, Hook. Hab. : Pangoa, Peru. \$.

P. murorum, Hook. Hab. . Andes of Ecuador, on trees at Guayrapata. $\$$.

P. microphyllinum, Mett. Hab.: Peru. \$.

P. onustum, Hook. Hab.: Andes of Ecuador, N. Granada, Venezuela. \$. 
Polypodium-continued.

P. (Goniophlebium) vacciniifolium. Fisch. \& Langsd. Hab.: Venezuela.

P. (Gonioph.) glaucophyllum, Kze. Hab.: N. Granada; Andes of Ecuador; Valley of Lloa, foot of Tunguragua and of Chimborazo.

P. (Gonioph.) Matthewsii, Mett. Hab.: Chacapoyas, Peru; Ecuador, near Pasto, and forests of the Eastern Andes. $\$$.

P. (Gonioph.) pubescens. Hook. \& Gr. Hab. : Andes of Peru. Hook. \& Gr. 1c. Fil. t. 18:. \$.

P. (Gronioph.) plesiosorum, Kunze (P. loriceum, L. in part.). Hab. : Venezuela.

P. (Gonioph.) lasiopus, Klotzsch. Hab.: Venezuela. \$.

P. (Gonioph.) loriceum, L. Hab. : Ecuador, Peru.

P. (Gonioph.) eleutherophlebium, Mett. Hab.: Venezuela. \$. $\$$ \$.

P. (Gonioph.) Chacapoyense, Hook. Hab.: Chacapoyas, Peru.

P. (Gonioph.) Surrucuchense, Hoo\%. Hab. : Ecuador, Venezuela, Surrucucha ; Tunguragua, on trees.

P. (Gonioph.) chnoodes, Spreng. (P. dissimile, Hook.). Hab.: Venezuela.

P. (Gonioph.) attenuatum, $H . B . K$. Hab. : Caripe, Venezuela, Ecuador.

P. (Gronioph.) neriifolium, Schk. Hab.: Caraccas, Venezuela, Ecuador, Peru.

P. (Gonioph.) cordatum, Kze. Hab.: Peru. $\$$

P. (Gonioph.) adnatum, Hook. Hab.: N. Granada, Ecuador, Galapagos.

P. (Gonioph.) fraxinifolium, Jacq. Hab. . Venezuela, N. Granada, Ecuador, Peru.

P. (Gonioph.) incanum, Swz. Hab.: throughout Tropical America.

P. (Gonioph.) lepidopteris, Kze. Hab.: Galapagos, Tarapota, E. Peru.

P. (Gonioph.) squamatum, L. Hab.: Venezuela, Andes of Ecuador, Chacapoyas, Peru.

P. (Phlebodium) nigripes, Hook. Hab.: Tovar, Venezuela. \$.

P. (Phleb.) aureum, L. Hab.: Ecuador, Galapagos, Venezuela.

P. (Phleb.) decumanum, Willd. Hab. : N. Granada, Tarapota, E. Peru.

P. (Campyloneuron) angustifolium, Swz. Hab.: N. Granada, Ecuador, Peru.

P. (Camp.) lucidum, Beyr. Hab.: Venezuela.

P. (Camp.) sphenodes, Kze. Hab.: N. Granada, Merida, Ecuador; descent of Molleturo, alt. 7,000 feet. $\$$.

P. (Camp.) coarctatum, Kunze. Hab. : Peru. \$.

P. (Camp.) lapathifolium, Lam. (P. fasciale, Willd.). Hab.: N. Granada, Venezuela, Ecuador.

P. (Camp.) repens, $L$. Hab.: Chimborazo; Tarapota.

P. (Camp.) Phyllitides, L. Hab. : Ecuador, foot of Chimborazo, alt. 3,000 feet.

P. (Camp.) decurrens, Raddi. Hab.: Venezuela, Tarapota, E. Peru. 
Polypodium-continued.

P. (Camp.) Fendleri, Eaton. Hab.: N. Granada, Venezuela. \$\$.

P. (Niphobolus) Americanum, Honk. Hab.: Andes of Ecuador, about the suburbs of Cuença, on walls and hedgebanks, in great abundance. $\$$

P. (Phymatodes) percussum, Cav. Hab. : N. Granada, Ecuador, Galapagos.

P. (Phym.) lanceolatum, Linn. (P. lepidotum, Willd.). Hab.: N. Granada, Ecuador, Peru, Tarapota.

P. (Phym.) stigmaticum, Presl. Hab.: Columbia. \$.

P. (Phym.) lycopodioides, Linn. Hab. : Ecuador, Peru.

P. (Phym.) fusco-punctatum, Hook. Hab. . Ecuador, ascent of Chimborazo, alt. 3,000 feet.

P. (Phym.) persicariæfolium, Schrad. Hab.: Ecuador; Guayaquil, on the trunks of Theobroma Cacao, 'pale green colour, as all ferns growing on Cacao trees are.'

P. (Phym.) crassifolium, Linn. Hab.: Peru, Ecuador.

P. (Plym.) bifrons, Hook. Hab. : Ecuador, on a tree by the river side near Archedona. \$\$.

P. (Pbym.) leucosporum, Klotzsch. Hab.: Venezuela.

\section{Jamesonia.}

J. imbricata, Hook. \& Grev. Hab. : N. Granada, Andes of Ecuador, Peru. Hook. \& Gr. Ic. Fil. t. 178.

\section{Nothochlæena.}

N. sinuata, Kaulf. Hab.: Ecuador, valley of the Andes, alt. 7,000 feet; Tucuman, on earthen walls; Baños, Andes of Peru.

N. ferruginea, Hook. Hab. : Peru, Ecuador, N. Granada, Santa Martha.

N. Brackeridgii, Baker. Hab. : Peru. \$.

N. squamosa, Baker (Cheilanthes, Hook.). Hab.: Peru.

N. (Cincinalis) sulphurea, $J$. Sm. (N. candida, Hook.). Hab. :

Peru; Huanaco, hot valleys of Ecuador; Galapagos.

N. (Cinc.) flavens, Mloore (Gymnogramme flavens, Klfs.). Hab. . Ecuador; Andes of Quindiu, alt. 4,000 feet; Loxa.

N. (Cinc.) nivea, Desv. Hab. : Ecuador, Peru, Huano, Loxa.

\section{Gymnogramme.}

G. (Leptogramme) asplenioides, Kaulf. Hab.. New Granada, Venezuela, Tarapota.

G. (Lept.) diplazoides, Desv. (G. rupestris, $\boldsymbol{K}_{\tilde{z} e .}$.). Hab. : Venezuela, N. Granada, Caraccas, Andes of Ecuador, Tarapota, Eastern Peru.

G. (Lept.) grandis, Baker. Hab. : N. Granada, Ecuador. \$.

G. (Lept.) gracilis, Heward. Hab.: Venezuela.

G. reniformis, Mart. Hab. : near Tarapota, E. Peru, on Mount Guayapurima, very rare. Hook. Second Cent. Ferns, t. 9.

G. rufa, Desv. Hab. : N. Granada, Venezuela, Tarapota.

G. tomentosa, Desv. Hab. : Peru.

G. angustifrons, Buker (G. elongata, Hook.). Hab.: Andes of Ecuador, at Surrucucho, near Cuença, alt 9,000 feet; Loxa, Peru; Andinarca, near Agapata. \$.

G. Lirsutula, Mett. Hab. : New Granada. \$

G. Karstenii, Mett. Hab.: New Granada. \$. 
Gymnogramme-continued.

G. incisa, Mart. et Lind. Hab. : Maraginta ; New Granada, alt. 8,000 feet. $\$$.

G. Mohriæformis, Mett. Hab.: St. Gavan, Peru. \$.

G. Ottonis, Klotzsch. Hab. : Venezuela, mountain regions in arid places. $\$$

G. Lindigii, Mett. Hab. : New Granada. \$.

G. ferruginea, Kunze. Hab. : hot dry banks, Peru.

G. Hookeri, J. Sm. Hab.: Andes of New Granada and Bolivia. $\$$.

G. aureo-nitens, Hook. Hab.: Peru. \$.

G. Matthewsii, Hook. Hab.: Peru. \$.

G. Warcewiczii, Mett. Hab.: New Granada. \$.

G. flabellata, Hook. Hab. : Ecuador ; Surrucucho, near Cuença, alt. 9,000 feet, on the ground amongst herbage. $\$$.

G. leptophylla, Desv. Hab. : Andes of Ecuador.

G. hirta, Desv. (G. laserpitiifolia, Kze). Hab.: Columbia, 'Tovar. $\$$.

G. flexuosa, Desv. Hab.: Venezuela; Sierra Nevada, alt. 7,000 feet; Ecuador, woods near Cuença, alt. 8-10,000 feet, growing on the ground; Peru.

G. Pearcii, Moore. Hab.: Peru. \$\$.

G. (Ceropteris) trifoliata, Desv. Hab. : Ecuador, Peru.

G. (Cerop.) triangularis, Kaulf. Hab. : hot valleys of Ecuador.

G. (Cerop.) calomelanos, Kaulf. Hab. : Peru.

G. (Cerop.) pulchella, Linden. Hab.: Venezuela. \$.

G. (Cerop.) sulphurea, Desv. Hab.: Venezuela.

G. (Selliguea) elongata, Hook. Hab.: Venezuela, Western Andes of Quito.

\section{Meniscinm.}

M. giganteum, Mett. Hab. . shady moist woods, St. Gavan, Peru. \$.

M. angustifolium, Willd. Hab. : Caraccas, Venezuela, T'Tarapota ; Eastern Peru.

M. serratum, Cav. Hab. : N. Granada. pota.

M. reticulatum, Swz. Hab.: Venezuela, Ecuador, Peru, Tara-

\section{Antrophyum.}

A. lineatum, Kaulf. Hab.: N. Granada, Andes of Quito.

A. lanceolatum, Kaulf. Hab.: N. Granada.

A. subsessile, Kunze. Hab.: N. Granada, Peru, Tarapota; Ecuador, Andes of Quito.

\section{vittaria.}

V. (Tæniopsis) stipitata, Kunze. Hab. : Columbia, Peru, Tarapota. $\$$.

V. (Tæniop.) lineata, Swz. Hab.: Peru, Ecuador, N. Granada.

V. (Tæniop.) remota, Fée. Hab. : New Granada.

\section{Trenitis.}

T, angustifolir, $R . B r$. Hab.: Now Granada, Ecuador, Galapagos.

T. furcata, W'illd. Hab.. Venezuela, Ocaña; Ecuador, near Guayequil, on Theobroma Cacao. 


\section{Hemionitis.}

H. palmata, Linn. Hab.: N. Granada, Tarapota, Peru.

\section{Acrostichum.}

A. petiolosum, Desv. Hab. : Ecuador and Peru. \$\$.

A. Tambillense, Hook. Hab. : Ecuador, sides of ravines; Tambillo, near Quito.

A. leptophyllum. Hab. : Venezuela.

A. conforme, Sus. Hab.: Venezuela, Andes of Peru, Ecuador.

A. flaccidum, Fée. Hab.: N. Granada.

A. Schlimense, Fée. (A. scandens, Bory.). Hab.: N. Granada, alt. 7,000 feet; Ecuador. $\$$.

A. stenopteris, Klotzsch. Hab. : Columbia, Venezuela. \$.

A. Lingua, Raddi (A. latifolium, Sw. in part.). Hab.: Venezuela, New Granada, Peru.

A. latifolium, Sw:. Hab. : Peru, Tarapota.

A. stramineum, Mett. Hab.: N. Granada. \$.

A. melanopus, Kze. Hab.: N. Granada. \$.

A. decoratum, Kze. Hab.: Peru ; in dease forests, Pampayaco.

A. Féei, Bory. Hab. Ecuador, Quito, Chimborazo.

A. cardiophyllum, Hook. Hab. . Andes of Quito, alt. 14,000 feet. \$. $\$$ \$.

A. squamipes, Hook. Hab.: N. Granada, Chacapoyas, Peru.

A. Lindeni, Bory. Hab. : Caraccas; Venezuela, alt. 7,500 feet; Ecuador; Cotopaxi, alt. 13,000 feet, and caves on the eastern Cordillera.

A. squarrosum, Klotzsch. Hab.: Columbia. \$.

A. Lloense, Hook. Hab. . Venezuela, N. Granada, Valley of Lloa, Ecuador, and temperate forests of the Andes; Peru.

A. heteromorphum, Klotzsch. Hab.: N. Granada, Ecuador, western side of Pichincha. $\$$.

A. Huacsaro, Ruiz. (A. Calaguala, Klotzsch). Hab.: Merida, Columbia, Peru. \$\$.

A. Gardnerianum, Fée. Hab.: Venezuela.

A. alpestre, Gardn. Hab.: N. Granada.

A. viscosum, Swz. Hab.: New Granada, Venezuela, Ecuador, Peru.

A. ciliatum, Presl. (A. Preslianum, Fée.). Hab. . Caraccas, Merida, Ecuador, Peru, Tarapota. \$. feet.

A. pilosum, H. B.K. Hab. : Columbia ; N. Andalusia, alt. 6,600

A. scolopendrifolium, Raddi. Hab.: Venezuela, New Granada, Ecuador, forests on the west side of Pichincha; Andes of Peru.

A. ovatum, Hook. Hab. . N. Granada; Ocaña, alt. 10-11,000 feet; Ecuador ; Cayambe, alt. 14,000 feet, and Cordillera of Pillaro, alt. 15,000 feet.

A. Matthewsii, Fée. Hab.: Peru, on trees, and in erevices of rocks; Andes of Ecuador.

A. acrocarpon, Mart. IIab. : New Granada; Ecuador, on Mount MLulmul, alt. 8-9,000 feet.

A. lepidotum, Willd. Hab.: N. Grauada, on Mount Azuay, alt. 13,000 feet; Venezuela, Truxilio and Merida; Ecuador, Pichincha, Peru. \$\$. 
Acrostichum-continued.

A. tectum, Willd. (A. rubiginosum, Fée.). Hab.: Columbia, Santa Marta, Ocaña; Peru.

A. Bellermanianum, Klotzsch. Hab.: N. Granada, Venezuela, Ecuador. \$.

A. muscosum, Swz. Hab.: Quitinian Andes.

A. auricomum, Kunze. Hab.: Peru.

A. cuspidatum, Willd. Hab. . Venezuela, Caraccas, Ecuador, Peru, Tarapota.

A. squamosum, Swz. Hab.: Venezuela, Ecuador, Valley of Lloa.

A. Sprucei, Baker. Hab. : foot of Mount Chimborazo. \$.

A. (Stenochlæna) sorbifolium, Linn. Hab. . Peru, Columbia.

A. (Polybotrya) plumbicaule, Baker. Hab.: Tarapota, Peru. \$.

A. (Polyb.) fractiseriale, Baker. Hab.: Monte Campana, North Peru. \$.

A. (Polyb.) pubens, Baker. Hab.: Peru. Peru.

A. (Polyb.) caudatum, Hook. Hab. . New Granada, Ecuador,

A. (Polyb.) osmundaceum, Hook. Hab. : New Granada, Ecuador.

A. (Polyb.) canaliculatum, Hook. Hab. : Venezuela. \$S.

A. (Polyb.) Lechlerianum, Hook. Hab. : Peru; St. Gavan, shady places. Near Tarapota. \$5. Hook. Second Cent. Ferns, t. 97.

A. (Rhipidopteris) flabellatum, $H . B . K$. Hab. . New Granada, Ecuador, Peru. \$.

A. (Rhip.) peltatum, Swz. Hab.: N, Granada, Ecuador, Peru.

A. (Rhip.) foeniculaceum, Hook. Hab.: Ecuador, forest of Esmeraldas, alt. 2,500 feet, on trunks of trees, foot of Chimborazo, alt. 3,000 feet, on stones. $\$$.

A. (Olfersia) cervinum, Swz. Hab. : Peru.

A. (Gymnopteris) oligarchicum, Baker. Hab. : North Peru. \$.

A. (Gym.) alienum, swa. Hab.: North Peru.

A. (Chrysodium) aureo-nitens, Hook. Hab.: Chatham Island, Galapagos. \$s. Hook. Cent. Ferns, t. 33.

A. (Chrys.) pachyphyllum, Kze. Hab. . Peru. \$\$.

A. (Chrys.) pandurifolium, Hook. Hab.: Andes of Peru, Mount Guayrapurima, near Tarapota; Chimborazo, alt. 3,000 feet. \$. Hook. Second Cent. Ferns, t. 87.

A. (Chrys.) Raddianum, Kze. Hab. : North Peru.

A. (Chrys.) serratifolium, Mert. Hab.: Venezuela, North Peru.

A. (Chrys.) Lindigii, Baker. Hab.: New Granada. \$.

A. (Chrys.) aureum, Linn. Hab.: Peru, Galapagos.

\section{osmunda.}

O. cinnamomea, Linn. Hab.: N. Granada.

schizza.

S. dichotoma, Swz. Hab.: Peru; Venezuela.

\section{Anemia.}

A. hirsuta, Swz. Hab. : Peru.

A. tomentosa, Swz. Hab.: Peru and Monte Video.

A. (Anemidictyon) Phyllitidis, Swz. Hab. : Peru.

\section{Iygodium.}

L. digitatum, Eaton (non Presl.). Hab. New Granada.

L. venustum, Swz. Hab. . Peru. 
Marattia.

M. (Eupodium) Kaulfussii, J. Sm. Hab.: Ecuador.

M. alata, Smith. Hab.: Venezuela. Grisebach.

\section{Danaea.}

D. trichomanoides, Spruce MSS. Hab. : Tarapota. \$.

D. humilis, Moore. Hab. : Ecuador. \$.

D, alata, Smith. Hab.: Venezuela. Grisebach.

D. Augustii, Karst. Hab.: Venezuela. \$\$.

D. Moritziana, Presl. Hab. : Columbia. $\$$.

D. elliptica, Sm. Hab.: Venezuela. Grisebach.

\section{Ophloglossum.}

O. reticulatum, Linn. Hab. : Peru.

\section{Botrychium.}

B. Lunaria, Swz. Hab. : New Granada.

\section{GUIANA, BRAZIL, PARAGUAY, URUGUAY.}

\section{Cleichenia.}

G. revoluta, $H . B . K$. Hab. : Brazil.

G. pubescens, $H$. $B$. $K$. (Nertensia immersa, $K l f$.). Hab. : Guiana, Brazil, 'Gongo Soco, St. Catherine's, and Porto Alegre.' C. Bunbury; Hook. \& Gr. Ic. Fil. t. 15

G. glaucescens, Willd. (Mertensia pectinata, Presl.). Hab. : British Guiana, Surinam, French ( Guiana, N. Brazil, Amazon; South Brazil, Diamond district; Bahia, Para; Rio de Janeiro, "very common on the hills, especially in the more open and rocky parts, where it forms dense entangled thickets (like Pteris aquilina) six feet high, and more. Island of St. Catherine.' C. Bumbury.

G. dichotoma, Willd. Hab.: Brazil, Diamond district; Bahia, Porto Alegre, Para.

\section{Cyathea.}

C. arborea, Sm. Hab.: Ilhios, Brazil.

C. Serra, Willd. Hab. : Brazil.

C. vestita, Mart. Hab. . Brazil, woods of the province of St. Sebastian and St. Paul ; Serra dos Piloes, Serra de Araripe. \$. C. hirtula, Mart. Hab. : Serro do Mar, province of Bahia, Brazil ; Guiana. \$.

C. Schanschin, Mart. Hab. . throughout a great portion of Brazil, especially in the provinces of St. Paul and Minas, Organ Mountains. 'Plentiful in moist shady lavines at Gongo Soco, and on the edges of streams among the wooded mountains. A beautiful tree-fern: I never could find any fruit.' C. Bunbury.

C. Gardneri, Hook. Hab.: near Arrial des Merces, Brazil, and Morro Velho.

C. Beyrichiana, Pr. Hab. : Rio Janeiro. \$.

\section{Femitelia.}

H. grandifolia, Spr. (H. obtusa, Klfs.). Hab. : Guiana, abundant. H. speciosa, Hook. Hab. : Para, Brazil.

H. platylepis, Hook. Hab.: near San Carlos, Rio Negro, tri- 
Hemitelia-continued.

butary of the Amazon, Brazil. \$. Hook. Second Cent. Ferns, t. 100.

H. Moricandiana, Kze. Hab. : British Guiana, Babia. \$.

H. Guianensis, Hook. Hab.: Para, Brazil; Guiana, and the whole valley of the Amazon.

\section{Alsophila.}

A. blechnoides, Hook. Hab. : woods in Guiana, Para, and Rio Negro, Brazil.

A. Tænitis, Hook. (Trichopteris, Pr.). Hab.: Brazil, frequent about Rio and on the Corcovado, St. Sebestian, Minas Geraes, and elsewhere. 'At Gongo Soco, in the same situations with the Cyathea, but much less abundant; also in ravines in the Itacolumi Mountain, Ouro Preto.' C. Bunbury. \$.

A. elegans, Mart. Hab. : woods of St. Paul and Minas Geraes, apparently rare. $\$$.

A. marginalis, $K l$. Hab. : British Guiana. \$.

A. Miersii, Hook. Hab. : Organ Mountains, Tejuco. \$.

A. procera, Klfs. (A. Hookeriana, Klotzsch.). Hab.: Brazil, Provinces of St. Paul, Minas, and Para; Island of St. Catherine; Organ Mountains and Tejuco.

A. Schiedeana, Pr. Hab.: B. Guiana, Amazon.

A. armata, Pr. Hab.: Tejuco, near Rio de Janeiro; 'Gongo Soco, in moist gullies in the forest.' C. Bunbury.

A. Gardneri, Hook. Hab. . woods, San Gaetano, Brazil.

A. ferox, Pr. Hab.: Guiana and Amazon; Porto Alegre, Brazil. \$\$

A. leucolepis, Mart. Hab. : Brazil, Province of Minas Geraes.

Para. atrovirens, Pr. Hab.: Rio Negro, Brazil, Province of St. Paul.

A. infesta, $K_{z e}$. (A. phalerata, Mart.). Hab.: Guiana, Brazil,

A. villosa, Pr. (A. Humboldtii, $K l$.). Hab. : Brazil, near Moro Velho and Serra do Frio.

A. plagiopteris, Mart. Hab. : S. Brazil, province of St. Paul. \$.

A. hirta, Klfs. Hab. . Brazil. \$.

A. nigra, Mtart. Hab. : Rio Negro, Brazil. \$.

A. radens, $K l f s$. Hab.: Brazil. \&.

A. pruinata, Klfs. Hab.: Porto Alegre, Brazil.

\section{Dicksonia.}

D. Sellowiana, Hook. Hab. Organ Mountains, Brazil ; Porto Alegre.

1). adiantoides, H. B. K. Hab.: Gongo Soco, Rio de Janeiro, Brazil.

J) cicutaria, Swartz. Hab.: Rio Janeiro.

D. rubiginosa, Kuulf. Hab. : Brazil, Rio Janeiro, Tejuca, Bahia.

\section{Eymenophyllum.}

H. asplenioides, Sw. Hab. Brazil.

H. abruptum, Hook. Hab. : Brazil.

H. polyanthos, Swartz. Hab.: Guiana, Surinam, Brazil.

H. caudiculatum, Mart. Hab.: Organ Mountrins, Brazil. 'Gongo Soco, in the darkest shade of the forest.' C. Bunbury. 
Hymenophyllum-continued.

H. hirsutum, Sw. Hab. : Organ Mountains, Brazil. Hook.\& Gr. Ic. Fil. t. 84.

H. ciliatum, Sw. Hab.: Surinam, Brazil, ' on old mossy trees, Gongo Soco, and on wet shady rocks on the Organ Mountains.'C. Bunbury. (H. Plumieri, Hook. \& Gr. Ic. Fil. t. 123.)

H. microcarpum, Desv. (H. Organense, Hook.). Hab.: moist shady rocks, Organ Mountains, Brazil.

H. sericeum, Sio. Hab. : Brazil, 'on wet shady rocks on the Organ Mountains.' C. Bunbury.

H. Tunbridgense, S'mith. Hab. . Organ Mountains, Brazil.

H. fucoides, Svartz. Hab.: summit of the Organ Mountains, Brazil. Hook. Cent. Ferns, t. 63.

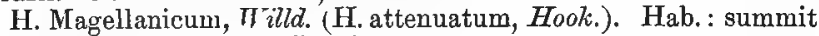
of the Organ Mountains, Brazil.

\section{Trichomanes.}

T. elegans, Rudge. Hab. : shady woods in Guiana; Valley of the Amazon. Hook. Gard. Ferns, t. 2.

T. botryoides, Kaulf. (T. nanum, Bory.). Hab. : Guiana.

T. spicatum, Hedw. Hab.: Guiana. Hook. Gard. Ferns, t. 60.

T. heterophyllum, H. B. K. (T. Spruceana, Hook. Cent. Ferns, t. 81). Hab. . San Gabriel-catingas; Rio Negro, a tributary of the Amazon.

T. punctatum, Poiret. Hab.: Guiana. Hook. \& Grev. Ic. Fil. t. 236 .

T. reptans, Swartz. Hab.: 'old mossy trunks of trees in the forest on the Corcovado, Rio de Janeiro.' C. Bunbury.

T. pusillum, Swartz. Hab.: Brazil. (T. quercifolium, Hook.\& Gr. Ic. Fil. t 115.)

T. Krausii, Hook. \& Gr. Hab.: Guiana. Hook, \& Gr. Ic. Fib. t. 149 .

T. sinuosum, Rich. (T. incisum, Kaulf.). Hab.: 'on trunks of arborescent Ferns (Cyathea Schanschin?) in the forest at Gongo Soco.' C. Benbury; Hook. \& Gr. Ic. Fil. t. Is.

T. Bancroftii, Hook. \& Gr. Hab.: British Guiana, Surinam, Brazil, Baña de Rio Negro and S. Gabriel. Hook. Gard. Ferns, t. 56 .

T. macilentum, V.D.B. Hab. : northern provinces of Brazil.

T. superbum, V.D.B. Hab.: British Guiana.

T. Kaulfussii, Hook. \& Gr. Hab.: Guiana and North Brazil. Hook. \& Gr. Ic. Fil. t. 10.

T. bicorne, Hook. Hab. : Barra do Rio Negro, on logs or roots of trees, in dense moist forests, Amazon, and at St. Gabriel, Rio Negro ; frequent on decayed logs on the Catingas. Hook. Cent. of Ferns, t. 82. \$.

T. brachypus, Kunze. Hab. British Guiana and South Brazil. (T. Ankersii, Parker ; Hook. \& Gr. Ic. Fil. t. 201.)

T. pyxidiferum, L. Hab. : Brazil.

T. radicans, Swartz (T. speciosum, Titld. ; Hymenophyllum rupestre, Raddi). Hab.: Brazil ; 'Gongo Soco, on old mossy trunks in the deepest shade of the forest ; Island of St. Sebastian.' C. Bunbury.

T. auriculatum, Blume. Hab. : Guiana. 
Trichomanes-continued.

'T. pinnatum, Swartz (T. floribundum, H. B. K.). Hab.: Guiana, Surinam, Brazil. Hook. Gard. Ferns, t. 8.

T. tenerum, Sprengel. (T. angustatum, Curm.). Hab.: Brazil, on Fern-tree stems with T. incisum. (T. sinuosum) Gongo Soco. C. Bunbury; Hook. \& Gr. Ic. Fil. t. 166.

T. rigidum, Swartz. Hab. . Brazil, 'under a dripping rock in a very shady spot, Gongo Soco ; Island of St. Sebastian.' C. Bunbury.

T. Leprieurii, Kunze. Hab.: Guiana and Brazil. Hook. Gard. Ferns, t. 11.

T. Sprucei, Baker. Hab.: North of Brazil. \$\$.

T. gemmatum, J. Smith (T. cellulosum, Sturn ; Hook. Second Cent. Ferns, t. 63). Hab.: Roraima, British Guiana, and Kunnuku Mountains. Valley of the Amazon, Bãna of Rio Negro and S. Carlos; North Brazil.

\section{Davallia.}

D. Imrayana, Hook. Hab.: Guiana.

D. Parkeri, Hook. Hab.: British Guiana. \$.

D. Saccoloma, Spreng. Hab.. Guiana; 'Brazil, Gongo Soco, in the thick shade of the forest.' C. Bunbury.

D. inæqualis, Kunze. Hab.: Brazil, Corcovado, and damp woods in the Organ Mountains.

D. Speluncæ, Baker (D. polypodioides, Hook.). Hab. : Brazil.

D. concinna, Schrad. (D. Schimperi, Hook.; D. thecifera, $H . B$. K. ; D. Lindeni, Hook.). Hab. : Brazil ; Organ Mountains, on the stem of an old tree.

D. bifida, Kaulf. Hab.: Brazil; Minas Geraes, Organ Mountains, St. Catherine. \$ook. \& Gr. Ic. Fil.t. 238.

\section{Iindsaya.}

L. falciformis, Hook. Hab.: Brit. Guiana. \$.

L. botrychioides, St. Hil. Hab. . Brazil. \$.

L. dubia, Spreng. Hab.: Guiank, and valley of the Amazon.

L. filiformis, Hook. Hab.: British Guiana. \$.

L. Catherinæ, Hook. Hab.: St. Catherine's, Brazil.

L. virescens, Swartz (L. Gardneri, Hook.). 'Hab.: Organ Mountains, Brazil, on a dry and shady bank; Island of St. Sebastian.' C. Bunbury.

L. trapeziformis, Dry. (L. quadrangularis, Raddi; L. horizontalis, Hook.; and L. Leprieurii, Hook.). Hab.: British Guiana, Rio Janeiro, Island of St. Catherine. 'Dry woods, Organ Mountains; Gongo Soco, in moist shady ravines.' C. Bunbury.

L. Guianensis, Dry. Hab. : Guiana, Kio Janeiro.

L. stricta, Dry. Hab.: Minas Geraes, Brazil. 'Gongo Soco and Cocaes, on broken rocky banks in the second-gronoth woods (capociras).' C. Bunbury.

L. pendula, Klotzsch. Hab.: British Guiana. \$\$.

L. Sprucei, Hook. MSS. Hab.: San Carlos, on the Rio Negro, North Brazil.

L. (Isoloma) reniformis, Dry. Hab. : Guiana and the Amazon Valley. $\$$

L. (Iso.) sagittata, Dry. Hab. : French Guiana. Hook. \& Gr. It. Fil. t. 87. 
Lindsaya-continued. $\$$.

L. (Scbizoloma) macrophylla, Kaulf. Hab. : Guiana and Brazil.

\section{Adiantum.}

A. Iunulatum, Burm. (A. dolabriforme, Hook.; A. deflectens, Mart.). Hab. : Minas Geraes, Organ Mountains, Brazil. 'Rio de Janeiro, in the woods beside the aqueduct.' C. Bunbury.

A. Kaulfussii, Kunze. Hab.: Guiana, Surinam, Amazon River.

A. calcareum, Gard. Hab.: clefts of calcareous rocks near Natividade, Province of Goyaz, Brazil. $\$$.

A. obliquum, Willd. Hab.: Guiana. 'Rio de Janeiro, in a very shady spot, in the deep narrow channel of a dry water-course, overhung by thick vegetation, in the woods beside the aqueduct, the elevation probably below 1,000 feet.' $C$. Bunbury.

A. subcordatum, Swartz. Hab. : Guiana and Brazil. \$.

A. intermedium, Swartz (A. fovearum, Raddi; A. triangulatum, Kaulf.). Hab. : Tropical America, frequent, especially in Brazil. Ilhios, Amazon, near Para. Guiana.

A. sinuosum, Gard. Hab.: Brazil, dry rocky places, near the summit of the Sierra de Natividade, province of Goyaz.

A. trapeziforme, L. (A. pentodactylon, Langsd. \& Fisch). Hab. : - Islands of St. Sebastian and St. Catherine. In the woods on the Serra do Mar, on the road from Rio de Janeiro to the Gold mines; also at Gongo Soco.' C'. Bunbury.

A. glaucescens, Klotzsch. Hab.: Guiana, and near Para, Brazil.

A. obtusum, Desv. Hab.: Guiana, Brazil. 'In thickets in the sandy plain between Rio de Janeiro and the Serra do Mar.' C. Bunbury.

A. hirtum, Klotzsch. Hab.. British Guiana, Surinam, Brazil, Ilhios, Para.

A. pectinatum, Kunze. Hab. : Brazil.

A. tetraphyllum, Willd. (A. prionophyllum, H. B. K.). Hab. : Surinam and Brazil ; Caripe.

A. lucidum, Srartz. IIab. : Brazil, Caripe, near Para.

A. Phyllitidis, J. Smith. Hab.: Guiana.

A. macrophyllum, Secertz. Hab. : Brazil. Hook. \& Gr. Ic. Fil. t. 132 ; Hook. Fil. Exot. t. 55.

A. rillosum, Linn. (A. falcatum, Sw.). Hab.: Guiana, Brazil.

A. pulverulentum, L. Hab. : Guiana, Brazil. 'TRio de Janeiro, in the thick dark woods on the Corcovado. Island of St. Sebastian.' C. Bunbury.

A. Capillus-veneris, $L$. Hab. : Amazon Valley.

A. Athiopicum, Lim. Hab. : Brazil.

A. cuneatum, Langs. \& Fisch. Hab. : Brazil, St. Catherine and Porto Alegre. Rio de Janeiro, 'abundant on the aqueduct.' $C$. Bunbury. And on Organ Mountaiı; Uruguay. \$.

A. digitatum, Presl. Hab.: Brazil.

A. tetragonum, Schrad. Hab.: Brazil. \$. Woods of Bahia, between Almada and Ferradas.

A. Hewardia, Kunze, Hab.: French Guiana. \$.

A. dolosum, Kuñe. Hab. . Guiana, Brazil.

A. olivaceum, Baker. Hab. : British Guiana. \$. 
Adiantum-continued.

A. Leprieurii, Hook. Hab. . Guiana; moist declivities of the mountain Matouri. $\$$.

\section{Eypolepis.}

H. repens, Presl. Hab. : Organ Mountains, Brazil, Ilhios.

H. nigrescens, Hook. Hab.: banks of the Amazon.

\section{Cheilanthes.}

Ch. monticola, Gardn. Hab.: Brazil, province of Goyaz; side of a narrow ravine on the summit of the Serra de Natividade. $\$$.

Ch. regularis, Mett. Hab.: Brazil. \$\$.

Ch. radiata, $\boldsymbol{R}$. Br. (Hypolepis radiata, Hook.). Hab.: ' Rio de Janeiro, in woods on the Corcovado.' C. Bunbury.

Ch. dichotoma, Swartz. Hab. : Brazil, Uruguay.

Ch. incisa, Kunze. Hab. : sonth of Brazil.

Ch. chlorophylla, Sw. (Hypolepis spectabilis, Link). Hab.: Brazil, common about Rio, Organ Mountains, Rio Grande. 'Estiva, on the road from Rio to Minas, in a rather dry wood; St. Catherine's and Porto Alegre.' C. Bunbury.

Ch. flexuosa, Kunze. Hab. : Brazil, Cape Goyan; Minas Geraes, in very sterile places.

\section{Cassebeera.}

C. triphylla, Kaulf. Hab.: S. Brazil; Monte Video; Porto Alegre.

C. pinnata, Kaulf. Hab.: S. Brazil, province of St. Catherine, Minas Geraes; stony shady places, province of St. Paul, Serra de Natividade.

C. gleichenioides, Gaud. Hab.: bushy rocky places, Diamond district, Brazil.

\section{Pellaea.}

P. columbina, Hook. (P. lomariacea, Hook. Sp.). Hab. : British Guiana; Brazil; old walls, Infecionado, Diamond district ; summit of the Organ Mountains.

P. geraniæfolia, Fée. Hab.: Brazil, 'Rio de Janeiro, on rocky banks at a rather low level, in the granite quarries near the city.' C. Bunbury; Hook. Cent. Ferns, t. 15.

\section{Pteris.}

Pt. litobrochioides, Klotzsch. Hab. . British Guiana and Amazon Valley.

Pt. quadriaurita, Retz. Hab.: 'Gongo Soco, Minas Geraes, Brazil ; in thick shady woods.' C. Bunbury.

Pt. pungens, Willd. Hab. : French Guiana.

Pt. heterophylla, Linn. Hab. : Brazil, Rio.

Pt. gracilis, Fée. Hab. : Brazil; 'Rio, in a very shady spot in the deep narrow channel of a dry water-course, overhung by thick vegetation in the woods beside the aqueduct, the elevation probably below 1,000 feet.' C. Bunbury.

Pt. deflexa, Link. Hab. : 'shady places about Rio, Gongo Soco; Minas Geraes, in thick shady woods." C. Bunbury.

Pt. aquilina, Linn. Hab.: Guiana; Brazil, abundant. Santa Rosa and Minas Geraes. 'This var. of Aquilina (Pt. arachnoides, Kaulf.) is very common in the provinces of Rio de Janeiro and Minas Geraes, especially in the capociras, or woods of second growth, 
Pteris-continued.

which grow up where the primeval forests have been burnt. St. Catherine's and Rio Grande.' C. Bunbury.

Pt. lomariacea, Kze. Hab.: British (Huiana; old walls, Infecionado, Diamond district, Brazil; summit of the Organ Mountains.

Pt. biaurita, $L$. Hab. : Brazil.

Pt. lonchophora, Metten. Hab. : Rio Janeiro.

Pt. sagittifolia, Raddi. Hab.: Brazil ; woods in the Mandiocea Mountains; rocks in shady woods in the Organ Mountains; Rio. Hook. Fil. Exot. t. 39.

Pt. ornithopus, Metten. Hab.: South Brazil.

Pt. palmata, Willd. (Pt. pedata, L. ; Pt. collina, Raddi). Hab. : Organ Nountains, Brazil.

Pt. pedata, Linn. Hab.: Islands of St. Sebastian and St. Catherine; and Porto Alegre, South Brazil. '(Pteris varians, var. of Pt. pedata) Ouro Preto, rocky banks on the mountains, at an elevation between 3,000 and 4,000 feet.' C. Bunbury.

Pt. splendens, Kaulf. Hab. : woods, Brazil; Organ Mountains and Arrial dos Minas.

Pt. denticulata, Swartz (Pt. Brasiliensis, Raddi). Hab. : Brazil, extremely common; Porto Alegre, Rio Grande, Ihios, San Gaetano, Minas Geraes. 'Rio de Janeiro, in thick woods on the Corcovado.' C. Bunbury.

Pt. decurrens, Presl. Hah. : 'Rio de Janeiro, in thick woods on the Corcovado.' C. Bunbury.

Pt. leptophylla, Sw. Hab.: Brazil, abundant about Rio. Hook. Gard. Ferns, t. 23.

Pt. incisa, Thunb. Hab. : Brazil.

Pt. Luschnathiana, Baker. Hab.: Brazil.

Pt. Kunzeana, Agardh. Hab. : French Guiana.

\section{Ceratopteris.}

C. thalictroides, Brong. Hab. : tropical and sub-tropical regions, growing in quiet or slightly current waters; French and British Guiana; Brazil, Bahia.

\section{Iomaria.}

I. attenuata, Willd. Hab. . Brazil, Organ Mountains.

L. divergens, Kunze (L. Plumieri, Desv.). Hab. : Brazil, shady wood by a small stream, Organ Mountains; B. Guiana.

L. alpina, Spreng. Hab. : S. Brazil.

L. procera, Spreng. Hab.: Organ Mountains, Brazil, Porto Alegre, Rio Grande do Sul.

L. Boryana, Trilld. (L. Magellanica, Desv.). Mab.: Brazil ; common near Tejuco, moist bushy places near San Pedro; boggy places near the summit of the Organ Mountains; Rio Grande do Sul, Uruguay. Hook. Gard. Ferns, t. 52.

L. volubilis, Hook. Hab. : Barra do Rio Negro, tributary to the Amazon, in thickets by streams, climbing to the height of twenty feet. Appun, British Guiana.

\section{Blechnum.}

B. Lanceola, Swartz. Hab. . Bogota, about Rio. Hook. Cent. Ferns, t. 70. 
Blechnum-continued.

B. asplenioides, Swartz. Hab.: Brazil, Rio Janeiro, Goyaz, Minas Geraes. 'Gongo Soco, on moist clayey banks.' C. Bunbury.

B. unilaterale, Willd. (B. polypodioides, Raddi). Hab. : Guiana and Brazil.

B. nitidum, Presl. Hab. : South Brazil.

B. Brasiliense, Desv. (B. Corcovadense, Raddi). Hab. : 'Brazil, abundant in the vicinity of Rio; St. Catherine's and Porto Alegre.' C. Bunbury.

B. longifolium, $H . B . K$. (B. gracile, Kaulf.). Hab. : "Gongo Soco.' C. Bunbury.

B. occidentale, Linn. Hab. : South Brazil, Rio Grande do Sul, St. Catherine's and Porto Alegre. 'Ouro Preto, Gongo Soco, \&c., common on damp walls and rocks.' C. Bunbury.

\section{Video.}

B. hastatum, Kaulf. Hab.: Rio Grande, Uruguay, Monte

B. serrulatum, Rich. Hab.: South Brazil, Porto Alegre, Ilha dos Marinheiros, Rio Grande do Sul. 'Botafogo, near Rio de Janeiro, among tall grass on low damp sandy ground, very near the level of the sea.'-C. Bunbury. Abundant in Guiana.

t. 15 .

B. volubile, Kaulf. Hab. : Guiana, Brazil. Hook. Gard. Ferns,

\section{Asplenium.}

A. angustum, Swartz (A. loriforme, Hook.). Hab. : Guiana and Brazil, near Para. Hook. Cent. Ferns, t. 26.

A. serratum, Linn. Hab.: 'Gongo"Soco, on a decaying tree in the woods.'-C. Bunbury. British Guinna. Schomburgh: Hook. Fil. Exot. t. 70 .

t. 11.

A. trilobum, Cav. Hab.: S. Brazil. Hook. Second Cent. Ferns,

A. monanthemum, Linn. Hab.: S. Brazil.

A. alatum, $H . B . K$. Hab. . Organ Mountains, Brazil.

A. salicifolium, Linn. Hab. : Rio Janeiro.

A. oligophyllum, Kaulf. Hab. : Brazil ; Gongo Soco, in Minas Geraes; Organ Mountains.

A. erectum, Bory. (A. Brasiliense, Rad.). Hab. : Rio de Janeiro and Porto Alegre (var. harpeodes, a large form with acuminate pinnæ). 'In very shady damp and dark spots in the forests; Gongo Soco.'-C. Bunbury. Guiana.

A. auriculatum, Swartz. Hab. : Guiana; Brazil, frequent.

A. anisophyllum, Kunze. Hab.: Brazil.

A. rhizophorum, Linn. (A. rachirhizon, Raddi). Hab. : Island of St. Sebastian, Gongo Soco, Rio.

A. Serra, Langs. \& Fisch. Hab. : Brazil, Guiana, Porto Alegre.

A. marinum, Linn. Hab.: Rio Grande do Sul. 'Banks of the Uruguay.' C. Bunbury.

A. auritum, Swartz. Hab. : Brazil, Rio, Gongo Soco, on decaying trees in the forest. C. Bunbury.

A. formosum, $\Pi^{i}$ ild. Hab. : Brazil, British Guiana. Hook. Fil. Exot. t. 16.

A. pulchellum, Raddi. Hab. : Brazil, Rio Janeiro.

A. lætum, Swartz. Hab. . valley of the Amazon, Porto Alegre, Guiana. 
Asplenium - continued.

A. mucronatum, Presl. Hab. : South Brazil, on the rough trunks of trees; Organ Mountains, Rio. Hook. Cent. Ferns, t. 17.

A. cuneatum, Lam. Hab. : British Guiana, Brazil.

A. scandicinum, Kaulf. Hab. : South Brazil.

A. fragrans, Swartz. Hab. : Brazil, St. Catherine's.

A. angustatum, Presl. Hab.: Brazil.

A. adiantoides, Raddi. Hab.: Brazil, Minas Geraes, St. Catherine's; on the trunks and branches of trees at Rio.

A. pseudo-nitidum, Raddi. Hab. : Brazil, Monte Frade, Organ Mountains; Rio, elevation 4,000 feet.

A. delicatulum, Presl. Hab.: Brazil.

A. (Atbyrium) decurtatum, Link. Iab.: Brazil. \$.

A. (Diplazium) plantagineum, Linn. Hab. . Brazil.

A. (Dipl.) grandifolium, Swz. Hab.: Ilhios, Brazil.

A. (Dipl.) celtidifolium, Kunze. Hab.: Brazil.

A. (Dipl.) arboreum, Willd. (D. auriculatum, Kaulf.). Hab. : Brazil.

A. (Dipl.) Shepherdi, Spreng. (A. striatum, Linn.). Hab.: Brazil, Rio, St. Sebastian.

A. (Dipl.) crenulatum, Baker. Hab. : Brazil.

A. (Dipl.) Lindbergii, Mett. Hab.: Brazil.

\section{scolopendrium.}

S. (Antigramme) Brasiliense, Kunze. Hab. : Rio de Janeiro.

S. (Anti.) plantagineum, Schrad. (S. Douglasii, Hook.). Hab.: Brazil, about Rio, Organ Mountains. Hook. \& Gr. Ic. Fil. t. 150.

\section{Didymochlosa.}

D. lunulata, Desv. (D. sinuosa). 'Hab. : Gongo Soco, in the thick forests.'-C. Bunbury. Porto Alegre; Brazil, in mountain woods, frequent. Hook. Gard. Ferns, t. 17.

\section{Aspidium.}

A. (Polystichum) aculeatum, Sw. Hab. : Brazil ; Gongo Soco, in the thick forests. C. Bunbury.

A. (Polyst.) Capense, Willd. (A. (Polyst.) coriaceum, Sw.). Hab.: British Guiana, Cayenne, Brazil, Minas Geraes. 'Isle of St. Catherine and Tlha dos Marinheiros Rio Grande. Darker coloured than the Cape plant, the leaflets narrower and more pointed, their serratures also more acute.' C. Bunbury.

A. (Cyrtomium) abbreviatum, Schrad. Hab. : British Guiana; Brazil ; Bahia, Ilhios, Rio, Corcovado.

A. (Cyclodium) meniscioides, Willd. Hab. . Brazil and Guiana.

A. plantagineum, Griseb. Hab. : French Guiana; Brazil, Amazon, Serra de Sā̄ Gabríel.

\section{Nephrodium.}

N. (Lastrea) subobliquatum, Baker. Hab.: Guiana and the Amazon Valley.

N. (Last.) chrysolobum, Fée. Hab. : Brazil.

N. (Last.) patens, Desv. Hab. : Brazil, 'on the aqueduct, Rio de Janeiro.' C. Bunbury.

N. (Last.) falciculatum, Desv. Hab. . Brazil; 'in very moist and shady spots on the Corcovado, Rio de Janeiro.' C. Bunbury. 
Nephrodium-continued.

N. (Last.) vestitum, Baker (N. Raddianum, Hook.). Hab.: Brazil, about Rio.

N. (Last.) Ctenitis, Baker. Hab. : Brazil. \$.

N. (Last.) Caripense, Hook. Hab. : Brazil.

N. (Last.) tetragonum, Hook. Hab. : Brazil.

N. (Last.) Leprieurii, Hook. Hab. : marshy woods of Central French Guiana.

N. (Last.) subfuscum, Baker. Hab. Cayenne. \$.

N. (Last.) conterminum, Desv. Hab. : Isle of St. Catherine and Rio Grande, Brazil. C. Bunbury.

N. (Last.) Kaulfussii, Hook. Hab. : Brazil.

N. (Last.) palustre, Baker. Hab. : Brazil. \$.

N. (Last.) Filix-mas, Rich. Hab. : Brazil, near the summit of the Organ Mountains.

N. (Last.) Mexicanum, Hook. Hab.: Brazil.

N. (Last.) subquinquefidum, Hook. (N. funestum, Hook.). Hab. : Guiana, Brazil. Hook. Cent. Ferns, t. 21.

N. (Last.) furcatum, Hook. Hab.: Organ Mountains, Brazil.

N. (Last.) acutum, Hook. Hab.: Brazil.

N. (Last.) denticulatum, Hook. (Aspid. Klotzschii, Hook. Cent. Ferns, t. 23). Hab.. British Guiana, Brazil ; Gongo Soco, under dripping shady rocks, in company with Trichomanes rigidum. $C$. Bunbury.

N. (Last.) effusum, Baker. Hab. : Brazil.

N. unitum, $R$. Br. (non Sieb.; N. propinquum, R. Br.). Hab.: Guiana, Brazil.

N. refractum, Hook. Hab. : Brazil. \$.

N. molle, Desv. Hab.: Cayenne, Brazil, Porto Alegre.

N. (Sagenia) cicutarium, Baker. Hab. : Brazil.

N. (Sagen.) macrophyllum, Baker (Aspid., Sw.). Hab. : St. Catherine's, S. Brazil.

\section{yphrolepis.}

N. cordifolia, Baker (N. tuberosa, Pr.; N. pectinata, Schott.). Hab.: Guiana, Brazil; 'St. Sebastian; moist rocky banks, Gongo Soco.' C. Bunbury.

N. exaltata, Schott. Hab.: Guiana, Brazil.

N. acuta, Presl. Hab.: Guiana.

\section{oleandra.}

O. nerifformis, Cav. Hab. : Guiana and Brazil.

O. nodosa, Presl. Hab.: Guiana.

\section{Polypodium.}

P. (Pheg.) Sancta Gabrieli, Hook. Hab.: San Gabriel, valley of the Amazon.

P. (Pheg.) Tijuccanum, Raddi. Hab.: Guiana and Brazil.

P. (Pheg.) pauciflorum, Hook. Hab. Surinam. \$.

P. (Pheg.) refulgens, Klotzsch. Hab. : Guiana.

P. (Pheg.) decussatum, Linn. Hab.: Brazil.

P. (Pheg.) caudatum, Kaulf. Hab.: Brazil.

P. (Pheg.) platyphyllum, Hook. Hab. : South Brazil.

P. (Pleg.) connexum, Kaulf. Hab.: Brazil. \$. 
Polypodium-continued.

P. (Pheg.) splendidum, Kaulf. (Polyp. formosum, Raddi). Hab. : Brazil, abundant.

P. (Goniopteris) diversifolium, $S w z$. (P. fraxinifolium, Jacq.). Hab.: South Brazil, about Rio, very abundant; Island of St. Sebastian.

P. (Goniopt.) crenatum, Siv. Hab.: Brazil ; Bahia. Hook. Fil. Exot. t. 84 .

Y. (Goniopt.) reptans, Swz. Hab.: Brazil.

P. (Goniopt.) tetragonum, Swz. Hab.: Guiana and Brazil.

P. (Grammitis) gramineum, Swz. Hab.: Guiana.

P. (Gram.) marginellum, Swz. Hab.: British Guiana.

P. furcatum, Mett. Hab.: trunks of trees, British Guiana; French Guiana, Janarate River; Cachocira, a tributary of the Amazon.

P. subdimidiatum, Baker. Hab.: British Guiana.

P. Organense, Mett. Hab. : Organ Mountains, Brazil. \$.

P. discolor, Hook. Hab.: British Guiana.

P. trichomanoides, Swz. Hab.: Guiana and Brazil.

P. jubæforme, Kaulf. Hab.: British Guiana, Surinam.

P. pilosissimum, Mart. et Gal. Hab.: Organ MLountains, Brazil.

P. cultratum, Willd. Hab. : Brazil.

P. pendulum, Sw. Hlab.: British Guiana, Brazil ; Aracas, on trees.

P. suspensum, Linn. Hab.: Brazil, Rio.

P. sublanosum, Hook. Hab.: Organ Mountains, Brazil.

P. rigescens, Bory. Hab.: Brazil. Hook. \& Gr. Ic. Fil. t. 216.

P. radicale, Moritz. Hab.: S. Brazil.

P. pulchrum, Mart. \& Gal. Hab.: Brazil.

P. taxifolium, Linn. (P. Plumula, H. B. K.). Hab. : S. Brazil, 'Ris de Janeiro, on mossy trunks of trees in the woods.' Porto Alegre, Rio Grande. C. Bunbury.

P. recurvatum, Kanlf. Hab. : Brazil. \$\$.

P. pectinatum, Linn. Hab.: Guiana, Brazil ; Island of St. Catherine. Hook. Gard. Ferns, t. 10.

P. Meridense, Klotzsch. Hab. : Brazil.

P. reclinatum, Brack. (P. semiadnatuin, Hook.). Hab.: Organ Mountains, Brazil.

P. firmum, Klotzsch. Hab.: Guiana.

P. achilleæfolium, Kaulf. Hab. . Brazil, on mossy stems of large trees; Orran Mountains.

P. (Goniophlebium) vaccinifolium, Fisch. \& Langsd. Hab.: Brazil ; Paraguny; Rio de Janeiro, on old trees and even in gardens; Purto Alegre. C. Bunbury.

P. (Goninph.) piloselloides, Linn. (P. aurisetum, Radd $)$. Hab.: Brazil ; ' Rio de Janeiro, on damp mossy rocks and trunks of trees, in thick woods on the Corcovado.' C. Bunbury.

P. (Gonioph.) Cathurinx, Fïch. \& Langsd. Hab. : Brazil. \$.

P. (Gonioph.) loriceum, Linn. Ilab.: Brazil. 'Upon trees in the Organ Mountains; Porto Alegre; Isle of St. Catherine.' $C$. Bunbury.

P. (Gonioph.) attenuatum, $H . B . K$. Hab.: Guiana and Brazil.

P. (Gonioph.) neriifolium, Schk. Hab.: 'Rio de Janeiro and 
Polypodium-continued.

Porto Alegre.' C. Bunbury.

P. (Gonioph.) adnatum, Hook. Hab.: British Guiana.

P. (Gonioph.) fraxinifolium, Jacq. Hab.: 'Rio de Janeiro, in thick shady woods on the Corcovado Mountain; Island of St. Sebastian.' C. Bunbury.

P.' (Goninph.) menisciifolium, Langsd. \& Fisch. Hab. : Brazil ; $\mathrm{S}$. Gabriel, on the Amazon; rocks in the falls (exhales a strong odour of Anthoxanthum in drying).

P. (Gonioph.) incanum, Sw. Hab.: Uruguay, 'Rio de Janeiro, common on mossy trees ; Island of St. Sebastian and Porto Alegre.' C. Bunbury.

P. (Gonioph.) lepidopteris, Kze. (P. sepultum, Kaulf.). Hab. : 'St. Catherine's and Porto Alegre, S. Brazil.'-C. Bunbury. British Guiana.

I'. (Phlebodium) aureum, Linn. Hab.: British Guiana, Surinam, ('var. sporadocarpum, Spr.) Gongo Soco ; Minas Geraes, on rocks.' C. Bunbury.

P. (Pbleb.) decumanum, Willd. Hab.: Guiana, Cayenne, Brazil. P. (Campyloneuron) angustifolium, Sw. Hab.: Guiana, Brazil, Rio Crande, Porto Alegre.

P. (Camp.) lucidum, Beyr. (P. nitidum, Kaulf.; Hook. Fil. Exot. t. 12). Hab.: Brazil, about Rio; Organ Mountains.

P. (Camp.) lapathifolium, Lam. (P. fasciale, Willd.). Hab.: Brazil, banks of the Marañon.

P. (Camp.) Phyllitidis, Linn. Hab.: Porto Alegre, Brazil. ' Organ Mountains and Gongo Soco, on mossy trunks of trees in the forests,' C. Bunjury.

P. (Camp.) decurrens, Raddi. Hab.: Brazil.

P. (Phymatodes) geninatum, Schrad. Hab. . Brazil. \$.

P. (Phym.) Schomburgkianum, Kze. Hab.: British Guiana; Brazil; Para, on trees; Rio Negro and S. Gabriel.

P. (Phym.) percussum, Cal. Hab.: Guiana, Brazil ; 'Organ Mountains, on mossy rocks and trees.' C. Bunbury; Hook. Fil. Exot. t. 59.

P. (Phym.) lanceolatum, Linn. (P. lepidotum, Willd.). Hab. . Guiana, Brazil.

P. (Phym.) Swartzii, Baker (P. serpens, Swz.). Hab. : Cayenne.

P. (Phym.) lycopodioides, Linn. Hab.: Guiana; S. Brazil, Porto Alegre.

P. (Phym.) Lindberoii, Mett. Hab.: Brazil. \$

P. (Phym.) persicariæfolium, Schrad. Hab.: Brazil ; Guiana, Orinoco.

P. (Phym.) crassifolium, Linn. Hab.: Brazil ("var. glaucum); Islands of St. Sebastian and St. Catherine and Porto Alegre.' (. Bunbury.

P. (Phym.) angustum, Mett. Hab. S. Brazil. 'Rio de Janeiro, on stumps of trees not far abore the sea level; Island of St. Sebastian and Porto Alegre.' C. Bunbury.

\section{Nothochlæna.}

N. Pohliana, Kmze. Hab.: Brazil, Serra de Natividade. \$.

N. eriophora, Fée. Ilab.. S. Brazil; shady clefts near the city of Oeiras. Hook. C'ent. Ferns, t. 9l. 


\section{Monogramme.}

M. graminioides, Baker ( II. furcatn, Desy.). Hab.: Brazil.

M. (Pleurogramme) seminuda, Baker (M. graminifolia, Hook.).

IIab. S. Brazil, Guiana.

M. (Pleur.) immersa, Fée. Hab.: Guiana.

\section{Gymnogramme.}

(т. (Leptogramme) asplenioides, Kaulf. Hab.: Brazil, frequent;

' Rio de Janeiro, on wet rocks on the Corcovado.' C. Bunbury.

C. (Lept.) villosa, Link. Hab. : Brazil. \$\$.

G. (Lept.) poly podioides, simeng. Hab. : Brazil, about Rio. \$.

G. (Lept.) diplazoides, Desv. (G. rupestris, Kze.). Hab. : South Brazil.

G. reniformis, Mart. Hab.: dense woods, Brazil : Mount Cupati, near the river Japura. Hook. Second Cent. Ferns, t. 9.

C. pumila, spreng. Hab. : moist woods, Guiana, Surinam, Para, and Amazon Talley. Hook. Second Cent. Ferns, t. 8.

G. rufa, Desv. Hab. : Brazil, rocky shores of the Amazon.

G. tomentosa, Desu. Hab.: Brazil, 'about Rio de Janeiro, in shady woods.' ('. Bumbury; Hook. Fil. Erot. t. 1:).

G. Schomburgkiana, Kze. Hab.: British Guiana. \$. Hook. Cent. Ferns, t. 92.

G. myriophylla, Swz. Hab. : Brazil, "Ouro Preto (or Villa Rica), Minas Geraes, on wet rocks.' C. Bunbury. \$8.

G. chrophylla, Desu. Hab.. Paraguay; Brazil. Hook. \& Gr. Ic. Fil. t. 45 .

G. (Ceropteris) trifoliata, Desv. Hab.: S. Brazil, 'Catas Altan, in Minas Geraes, -in marshy ground, not shaded, in the valley of a small stream.' C. B'mbury.

G. (Cerop.) calomelanos, Kaulf. Hab. : 'Rio de Janeirn, Gongo Snco, Sc., very common; among rocks, often in places exposed to the sum; Island of St. Catherine and Porto Alegre, Rio Griude.' C. Bumbury; Hook. Crard. Frns, t. 50.

\section{Meniscium.}

G. (Selliguea) elougata, Hook. Hab.. Surinam and Brazil.

M. serratum, Cav. Hah.: Guiana, Brazil; Surinam.

M. reticulatum, Sus. Hab. : Brazil.

\section{Antrophyum.}

A. lineatun, Kanlf. Hab. : Brazil.

A. Cayennense, Fualf. Hab.: Guiana and the Amazon Valley, San Gabriel.

\section{Vittaria.}

V. (Treniopsis) scabrida, Klotzsch. Hab. : Brazil.

V. (Treniop.) Gardneriana, Fée. Hab. : Organ Mountains, Brazil.

T. (Treniop.) lineata, Swz. IIab.: Guiana; Porto Alegre.

\section{Tanitis.}

T. angustifolia, R. Br. Hab.: Guiana, Brazil.

T. furcata, Willd. Hab.: Guiana, abundant; Amazon Valley.

\section{Fremionitis.}

II. (Anetium) citrifolia, Hook. Hab. common throughout Trnpical Anurica, frequently growing pendulous from palm stems; Amazons and Para. 
Acrostichum.

A. simplex, Swz. Hab.: Guiana, Brazil, Bara de Rio Negro.

A. nigrescens, Hook. Hab.: Brit. Guiana, Roraima. \$.

A. leptophyllum, Fée. Hab.: Brazil.

A. Haccidum, Fee. Hab. : Guiana, Brazil, San Gabriel.

A. Burchellii, Buker. Hab. . Brazil. $\$$.

A. Herninieri, Bory, Ilab. : Guiana, Brazil.

A. luridum, Fée. (A. Schomburgkii, Hook. in part.). Hab.: Guiana.

1. latifolium, Swz. Hab.: Brazil.

A. hybridum, Bory. Hab. : Brizil.

A. decoratum, Kze. Hab.: Guiana.

A. Lindeni, Bory. Hab. : Brazil.

A. Gardnerianum, Fée. Hab.: stems and branches of trees, Oruran Mountains, Brazil.

A. alpestre, Grardn. Hab. : Organ Mountains, Brazil.

A. lineare, Fée. Hab. : Brazil. $\$$.

A. viscosum, Swz. Hab.. Brazil, San Gabriel; Porto Alegre, South Brazil.

A. apodum, Kaulf. Hab.: Brazil, Para, San Gabriel. Hook. \& Gr. Ic. Fil. t. 99.

A. scolopendrifolium, Raddi. Hab. : Surinam, Brazil. Brazil.

A. ovatum, Hook. Hab.: summit of the Organ Mountains,

A. acrocarpon, Mrrt. Hab. : Brazil, Minas Geraes.

A. strictum, Raddi. Hab.: Brazil.

A. muacosum, Sw. (A. Langsdorfii, Hook. \&. Gr.). Hab. : Brazil, ' Gongo Soco, Minas Geraes, among bushes, on rocks of iron-stone, on the steep fuce of a mountain probably more than 3,000 feet above the sea.' C. Bunbury.

A. auricomum, Kunze. Hab.: S. Gabriel, Amazon.

A. (Stenochlæna) sorbifolium, Linn. Hab. . S. Biazil, Guiana.

A. (Polybotrya) pubens, Baker. Hab.: Brazil.

A. (Polyb.) acuminatum, Hook, Hab.: Brazil. \$.

A. (Polyb.) caudatum, Hook. Hab.: Guiana, Amazon, Brazil.

A. (Polyb.) osmundaceum, Hook. Hab.: Brazil.

A. (Rhipidopteris) peltatum, Swz. Hab.: Griana, Brazil.

A. (Olfersia) cervinum, Swz. (Olfersia Corcovadensis, Raddi). Hab. . South Brazil ; Porto Alegre; Corcovado Mountains, in shady woods; Organ Mountains. Hook. Fil. E.rot. t. 43.

A. (Soromanes) Cænopteris, Kze. Hab.: S. Brazil.

A. (Gymnopteris) alienum, Swz. (G. semipinnatifida, Fée.). Hab. : Amazon Talley; French Guiana; rocky rivulet in the Serra de Sauto Ciabriel. Hook. Cent. Ferns, t. 71, 72.

A. (Gymn.) nicotianæfolium, Swz. Hab.: A mazon, Valley; British Guiana, near Para, Brazil. Hook. Gard. Ferns, t. 26.

A. (Chrysodium) Raddianum, $K z e$. Hab. : Brazil.

A. (Chrys.) serratifolium, Mert. Hab. : Brazil, frequent; Islands of St. Catherine, St. Sebastian.

\section{osmunda.}

A. (Chrys.) aureum, Linn. Hab. . S. Brazil.

O. cinnamomea, Linn. Hab. : S. Brazil.

O. regalis, Linn. Hah. : 'Ilha dos Marinheiros, Rio Grande, and banks of the Uruguay (var. O. gracilis, Link). In marshy ground, 
Osmunda-continued.

by the side of a pool, in open hill-pastures (campos); Gnngo Soco, and in similar situations on the Organ Mountains, at eleration of about 3,000 feet; banks of the Uruguay, where some of the specimens are hardly distinguisbable from the Forth American form, called O. spectabilis.' C. Bunbwy.

\section{Schizzea.}

S. incurrata, Schk. Hab.: Guiana and North Brazil.

S. (Jophidium) fluminensis, Miers. Hab. : Guiana and Brazil.

S. (Loph.) Sprucei, Hook, MSS. Hab. : banks of the Rio Negro.

S. (Loph.) elegans, Sw. Hab. : Brazil. Hook, Gard. Ferns, t. 54.

S. (Actinostachys) pennula, Suz. Hab.: Rio Janeiro; Guiana.

\section{Anemia.}

A. Gardneri, Hook. Hab. . South Brazil.

A. filiformis, Presl. Hab. : Brazil.

A. oblongifolia, Swo. Hab.: Brazil.

A. rotundifolia, Schrad. (A. radicans, $\beta$. Raddi). Hab.: South Brazil. $\$$.

A. caudata, Kaulf. (A. radicans, Raddi). Hab.: Brazil. \$.

A. mandioccana, Raddi. Hab. . Brazil, 'in the forest on the Corcorado, Rio de Janeiro.' C. Bunbury; Hook. Gurd. Fe'sls, t. 36. A. collina, Raddi. Hab. : Brazil, hills in the viciuity of Rio Janeiro; Rahia; Serra do Mar. Mook. Fil. Exot, t. 1.

A. glareosa, Gardn. Hab. : S. Brazil.

1. Breuteliana, Presl. (A. mandioccana, Hook. Sp. Fil.). Hab.: Birazil.

A. Langsdorffiana, Presl. Hab. : S. Brazil.

A. hirsuta, Sux. Hab. : S. Brazil.

A. tomeutosa, Suz. (A. flexuosa, Sw., Raddi; A. fulra, Suz.). Hab.: 'Rio de Janeiro, common among granitic rocks, beside the aqueduct, often in very sunny places; Horto Alegre.'-C. Bunlury. British Guiana. Hook. Fil. Exot. t. 30, and t. 26.

A. trichoriza, Gardn. Hab.: S. Brazil.

A. rutæfolia, Mart. Hab.. S. Brazil.

A. adiantifolia, Sw: Hab.: Bahia.

A. (Anemidictyon) Tweediana, Hook. Hab.: Tucuman and Uruguay, and South Brazil. Hook. Cent. Fums, t. 6.

A. (Anenid.) Schraderiana, Mett. Hab. : S. Brazil.

A. (Anemid.) Phyllitidis, Swo. Hab.: S. Brazil, 'in mountain woods near Valença, Minas Geraes; St. Catherine's and Ports Alegre (A. fraxinifolia, Raddi). In the woods on the Corcovado, Rio de Janeiro.' ('. Bunbury.

A. (Coptophyllum) dichotoma, Gardn. Hab.: Brazil.

A. (Copt.) willefolia, Gardn. Hab.: Villa de Arrayas, Goyaz; Brazil.

\section{Trochopteris.}

\section{Iygodium.}

T elegans, Gardn. Hab.: Serra de Natividade, Goyaz, Brazil.

L. venustum, Swz. Hab.: Brazil.

I. volubile, Sicz. (L. hastatum, Mart.). Hab. : Brazil, ' in thickets in the Laranjeiras valley, near the foot of the Corcovado; Rio de Janeiro, in Inw and rather dry woods (of second growth) on the lower parts of the hills.' C. Bunbury. 
Marattia.

M. (Gymnotheca) cicutæfolia, Kaulf. Hab.: Brazil ; Gongo Soco, in damp shady wooded ravines.' C. Bunbury.

M. (Eupodium) Kaulfussii, $J$, Sm, Hab. : Rio Janeiro. Hook. Second Cent. Ferns, t. 95.

\section{Dangea.}

D. simplicifolia, Rudge. Hab. : Guiana and N. Brazil.

D. trifoliata, Reich. Hab. : Guiana.

I). Leprieurii, Kze. Hab. : Guiana and Amazon Valley.

I. elliptica, Sm. Hab.: S. Brazil; 'Gongo Soco, in the thick forest.' C. Bunbury.

D. nodosa, Sm. Hab. : Brazil.

\section{Ophioglossum.}

O. nudicaule, L. fil. Hab.: Brazil.

O. reticulatum, Linn. Hab.: S. Brazil.

O. (Cheiroglossa) palmatum, Linn. Hab.: S. Brazil.

\section{CHILI, LA PLATA, PATAGONIA, FALKLAND ISLES.}

Gleichenia.

G. pedalis, Klfs. Hab. : Chili, Valdivia and Chiloe; Juan Fernandez.

G. cryptocarpa, Hook. Hab. : plains near Los Andes, Province of Valdivia, and Chiloe; Falkland Islands.

G. quadripartita, Hook. (G. acutifolia, Hook. Sp.). Hab. : Straits of Magellan, Port Famine.

G. glaucescens, Willd, (G. pectinata, Presl.). Hab. : Chili.

Thy rsopteris.

\section{Alsophila.}

T. elegans, Kze. Hab. : Juan Fernandez. \$.

A. villosa, Pr. (A. Humboldtii, Kl.). Hab. : S. Chili. divia.

A. pruinata, Kaulf. Hab. : Chili, frequent; Concepçion, Val-

\section{Woodsia.}

W. incisa, Gill. Hab. : Argentine Republic, Mendoza, San Luis, Cordova, Villa Vicenzia.

\section{Dicksonia.}

D. Berteroana, Hool. Hab.: thickly wooded places on the elevated mountains of Juan Fernandez.

\section{Eymenophylum.}

H. cruentum, Cav. Hab. : Chili, Valdivia woods; Chiloe, on trunles of trees. $\$$.

H. rarum, R. $\not B r$. Hab. : Chiloe, Tierra del Fuego.

H. polyanthos, Swz. Hab.: S. Chili, Juan Fernandez.

H. caudiculatum, Mart. Hab.: Chiloe.

H. fuciforme, Swo. Hab.: Chiloe; shady wonds of Valdivia ; trunks of trees in mountain woods of Juan Fernandez.

H. Chiloense, Hook. Hab.: S. Chili, and Island of Chiloe; Valdivia, on trunks of trees in woods.

H. Franklinianum, Colenso (H. subtilissimum, Kze.). Hab.: Chili, Chiloe, and Juan Fernandez. 
Hymenophyllum-continued.

H. Tunbridgense, Smith. Hab.: Chili, Staten Land, Cape Horn, Falkland Isles.

H. Serra, Presl. Hab. : Chili and Chiloe.

H. secundum, Hook. \& Gr. Hab.: Staten Land and Hermite Island, Cape Horn. Hook. \& Gr. Ic. Fil. t. 133. \$.

H. pectinatum, Cav. Hab.: San Carlos de Chiloe, woods in Valdivia; Chonos Archipelago. \$.

H. Magellanicum, Willd. (H. attenuatum, Hook.). Hab. : Chili, Chiloe.

H. Bridgesii, Hook. Hab.: Chili and Chiloe; Valdivia, on trunlss of trees. $\$$.

H. tortuosum, Hook. \& Gr. Hab.: Chili, Valdivia; Staten Land, Tierra del Fuego; Patagonia. Hook. \& Gr. Ic. Fil. t. 129. \$. H. dichotonum, Cav. Hab. : Chiloe, Juan Fernandez.

\section{Trichomanes.}

T. cæspitosum, Hook. Hab.: Staten Land, Falkland Islands; rocks and trunks of trees, Hermite Island, Cape Horn.-J. $D$. Hooker. Chiloe. \$.

T. exsectum, Kunze. Hab.: S. Chili, Chiloe, Valdivia, Juen Fernandez. $\$ \$$.

\section{Adiantum.}

A. tenerum, Swartz. Hab.: Juan Fernandez.

A. excisum, Kunze. Hab. : Chili, Valparaiso. \$.

\section{Cassebeera.}

C. triphylla, Kaulf. Hab.: Buenos Ayres.

\section{Pellæa.}

P. andromedæfolia, Fée. Hab.: Chilian Andes.

P ternifolia, Fée. Hab. : Andes of Chili, Sierra de Achira and El Moro.

P. glauca, J. Sm. (P. hirsuta, Hook.). Hab. : Andes of Chili, at La Guardia, and at Antuco, in South Chili ; near Valparaiso, in marshes.

\section{Pteris.}

Pt. marattiæfolia, Hook. Hab.: Chili and Chiloe. \$.

Pt. Chilensis, Desv. Hab.: Chili, Valparaiso, and Concepçion; Juan Fernandez. \$.

Pt. comans, Forst. (Pt. Berteroana, Ag.). Hab.: shady woods, Juan Fernandez.

Pt. incisa, Thunb. Hab.: Juan Fernandez; Chili.

\section{Iomaria.}

L. L'Herminieri, Bory. Hab. . Chili.

L. blechnoides, Bory. Hab.: Chili, Concepçion, Talcaluano, Province of Valdivia; Juan Fernandez. \$\$.

L. aspera, Klotzsch. Hab. : Chili and Cbiloe. \$.

L. alpina, Spreng. Hab.: Valdivia, as far north as Concepçion, in Chili; Cordillera of Colchagua, and Juan Fernandez. Plentiful in Temperate South America; abundant in the Straits of Magellan, as well as on Hermite Island, Cape Horn, and along the west coast of Patagonia; Staten Land; Falkland Islands. Fil. Exot. t. 32.

L. procera, Spreng. Hab.: Mendoza, Chili, Juan Fernandez. 
Lomaria-continued.

L. Boryana, Willl. (L. Magellanica, Desv.). Hab.: Straits of Magellan and Tierra del Fuego, and Falkland Islands, very abundant; Hermite Island, Cape Horn.

L. Germainii, Hook. Hab. : Chili. \$.

\section{Blechnum.}

B. occidentale, Linn. Hab.: Chili.

B. arcuatum, C. Gay. Ilab.: Chiloe, South Chili, Valdivia, from the coast to the elevation of 2,000 feet on the Andes. $\$$.

B. hastatum, Kaulf. Hab. : Chili, Concepçion to Valdivia; Juan Fernandez; Buenos Ayres.

\section{Asplenium.}

A. trilobum, Cav. Hab.: S. Chili and Chiloe.

A. monanthemum, Linn. Hab.: Chili, on trunks of trees, Laguna de Ranco.

A. macrosorum, Bertero. Hab.: on trees in thick woods, Juan Fernandez. $\$$.

A. obtusatum, Forst. Hab. : Chili, Talcuahano, Valdivia.

A. Magellanicum, Kaulf. Hab. : Juan Fernandez and Isle of Massa Fuera; Cape Horn, Port Famine, Hermite Island. Hook. \& Gr. Ic. Fil. t. 180 . \$\$.

A. divaricatum, Kunze. Hab. : South Chili.

A. Gibertianum, Hook, Hab.: Assumption, State of Paraguay, 650 miles above Buenos Ayres. Hook. Second Cent. Ferns, t. 22. \$8.

\section{Aspidium.}

A. (Polyst.) aculeatum, $S w$. (Aspid. Moritzianum, $K l$.). Hab.: Valdivia, Concepçion, Chili, Chiloe, Juan Fernandez; Falkland Islands ; Tierra del Fuego.

A. (Polyst.) mohrioides, Bory. Hab.: South Chili, Antuco, summit of the Pico de Pilque, Cordillera de Chillon of Zalcareque, Province of Colchagua and of Aculco; Falkland Islands; Patagonia and Straits of Magellan; Orange Harbour, Tierra del Fuego. $\$$.

A. (Polyst.) Capense, Willd. (A. (Polyst.) coriaceum, Sw.). Hab. : Sierra de Tordil, Argentine Republic; Chili, frequent, especially in the south; Valdivia, Patagonia, Port St. Elena, Port Desire.

A. (Polyst.) flexum, Kinnze (A. Berteroanum, Colla). Hab. : Juan Fernandez, caudex creeping over stones and the roots of trees in woods. \$5.

A. (Polyst.) multifidum, Mett. Hab.: Chili. \$.

\section{Nepbrodium.}

N. (Lastrea) patens, Desv. Hab.: Chili.

N. (Last.) concinnum, Baker. Hab.: Chili.

\section{Nephrolepis.}

N. altescandens, Baker (Polyp., Colla). Hab.: Juan Fernandez.

\section{Polypodium.}

P. (Pheg.) punctatum, Thunb. (P. rugulosum, Labill.). Hab.: Chili, Chiloe, Valdivia, Juan Fernandez.

P. jungermannioides, Klotzsch. Hab. : Chili.

P. (Grammitis) australe, Mett. Hab. : Lima and extreme south of South America, Straits of Magellan, Fort Famine; Hermite 
Polypodium-continued.

Islind, trunks of trees close to the sea; ascends to 1,500 feet; Falkland Islands.

P. serrulatum, Mett. (Xiphopteris Jamesoni, Hook. Second Cent. Ferns, t. 14). Hab.: Juan Fernandez.

P. trichomanoides, Sicz. Hab.: Juan Fernandez.

P. macrocarpum, Presl. Hab.: Bolivia and Chili. \$

P. rigescens, Bory. Hab. . Chili. Hook. \& Gr. Ic. Fil. t. 216.

P. (Goniophlebium) trilobum, Cav. Hab.: Chili. \$.

P. (Gonioph.) translucens, Kinze. Hab.: Juan Fernandez. \$.

P. (Gonioph.) loricenm, L. Hab.: Chili.

P. (Gonioph.) incanum, Sw. Hab.: Atacama, in Chili.

P. (Phymatodes) lancenlatum, Linn. (P. lepidotum, Willd.). Hab.: Juan Fernandez and Araucana in Chili.

\section{Nothochlzena.}

N. sinuata, Kaulf. Hab.: Chili.

N, ferruginea, Hook. Hab. : Chili.

N. mollis, Kunze. Hab. : Chili, Valparaiso, Coquimbo.

N. hypoleuca, Kunze. Hab. : Chili, Sierra de Portezuela, southern extremity of the Cordora range, and Tucuman, on earthen walls. $\$$.

N. tenera, Gillies. Hab. : Mendoza, eastern foot of the Chilian Andes, and baths of Villa Vicenzia; Bolivia, and on earth walls about Tucuman. \$.

N. (Cincinalis) sulphurea, J. Sm. (N. candida, Hook.). Hab. : Chilian Andes.

N. (Cine.) Chilensis, Hook. Hab. : Juan Fernandez. \$.

\section{Gymnogramme.}

G. Pozoi, Kanze (G. papaverifolia, Kunze). Hab. : Chili, Leona Mountains, San Iago, Cordillera, Valparaiso.

G. chærophylla, Desu. Hab.: Buenos Ayres.

G. (Selliguea) elongata, Flook. Hab. : Juan Fernandez.

\section{Acrostichum.}

\section{Schizæa.}

A. conforme, Sw. Hab. : Chili.

S. australis, Gaud. Hab. : Falkland Islands.

S. fistulosa, Labill. (S. Valdiviana, Phil.). Hab. : Cbili.

\section{Ophioglossum.}

O. bulbosum, Michx. Hab.: Chili.

\section{Botrychium.}

B. Lunaria. Hab. : Patagonia. 


\section{T A B L E S}

SHOWING

TIIE GEOGRAPHICAL DISTRIBUTION OF FERNS.

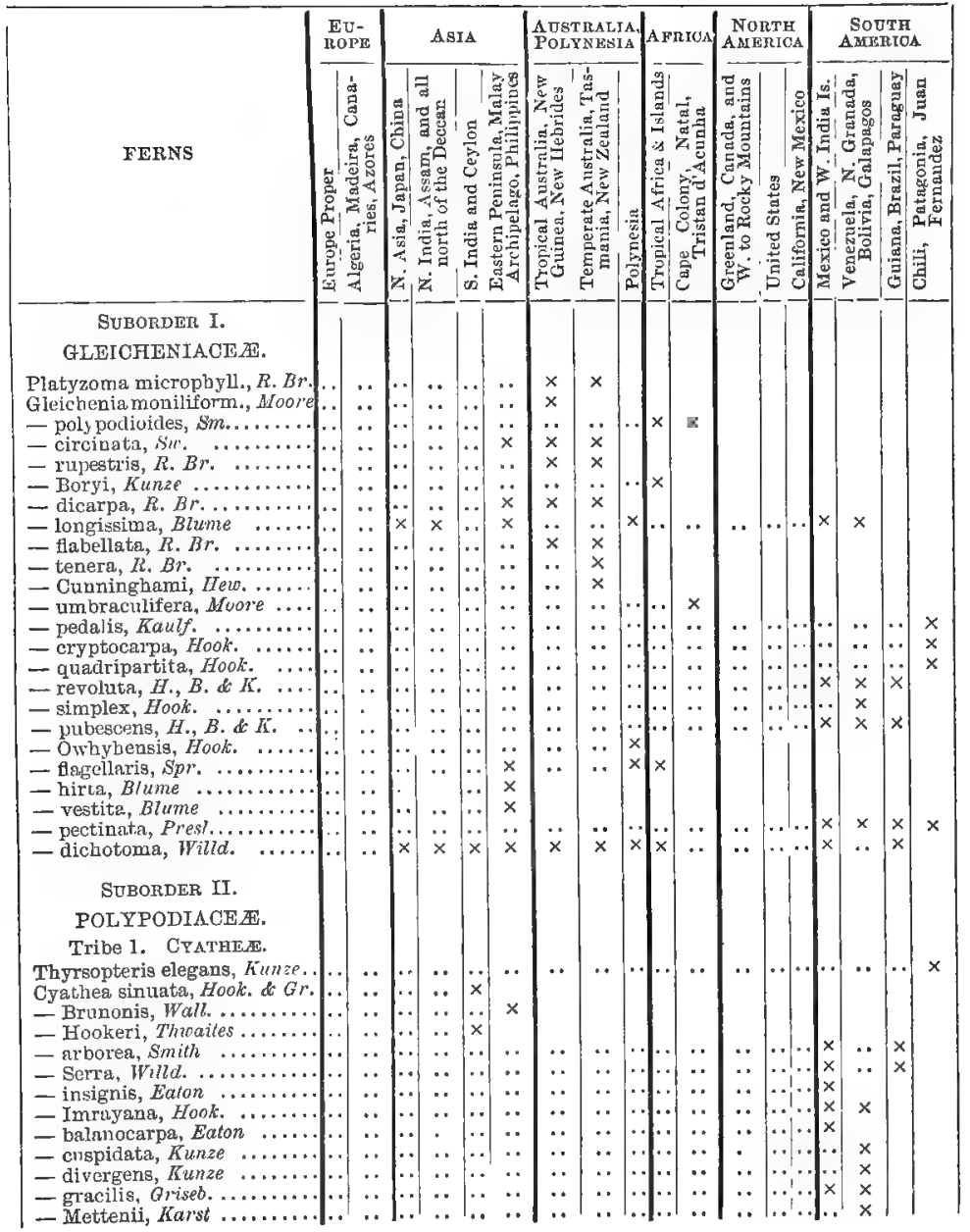




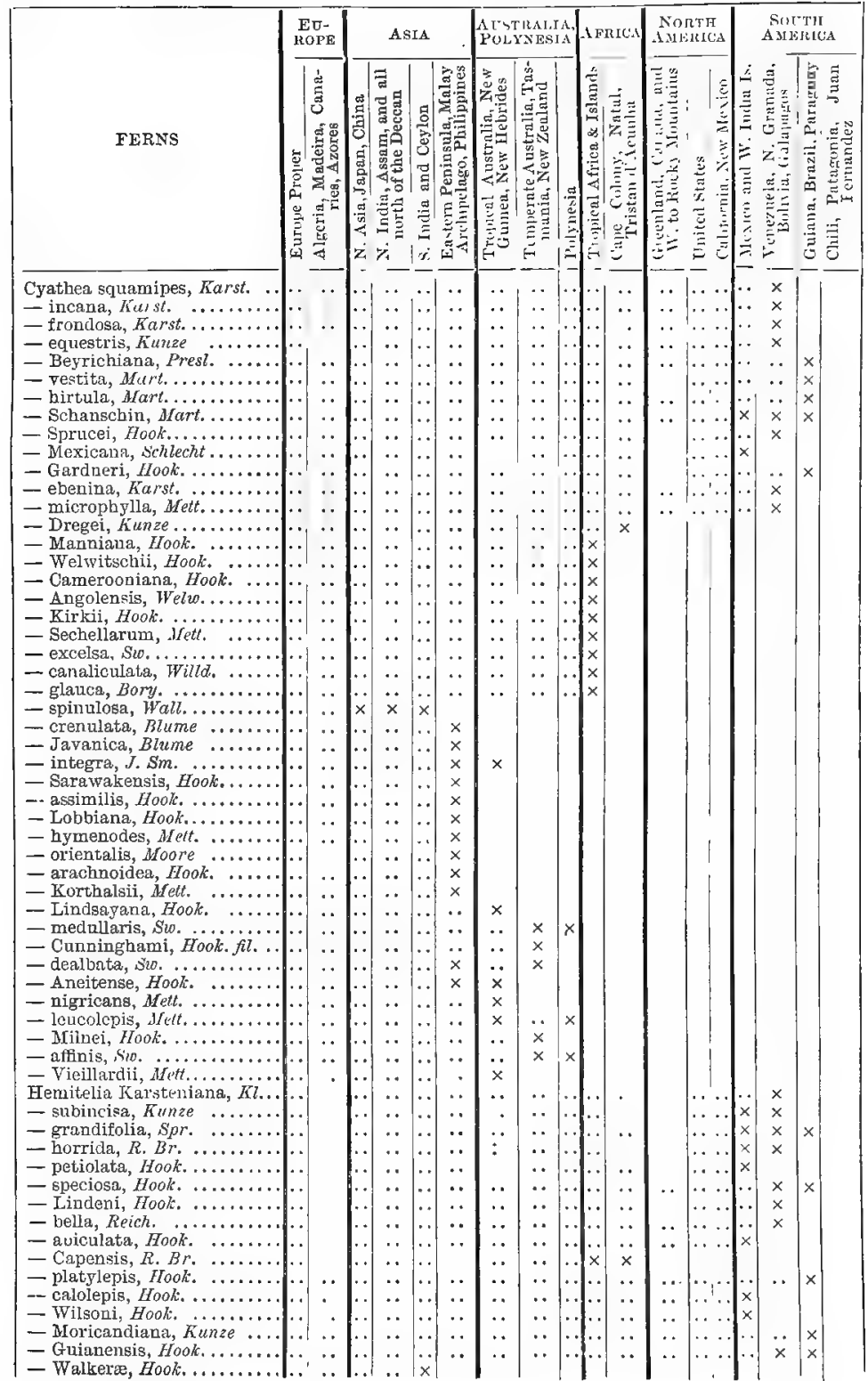




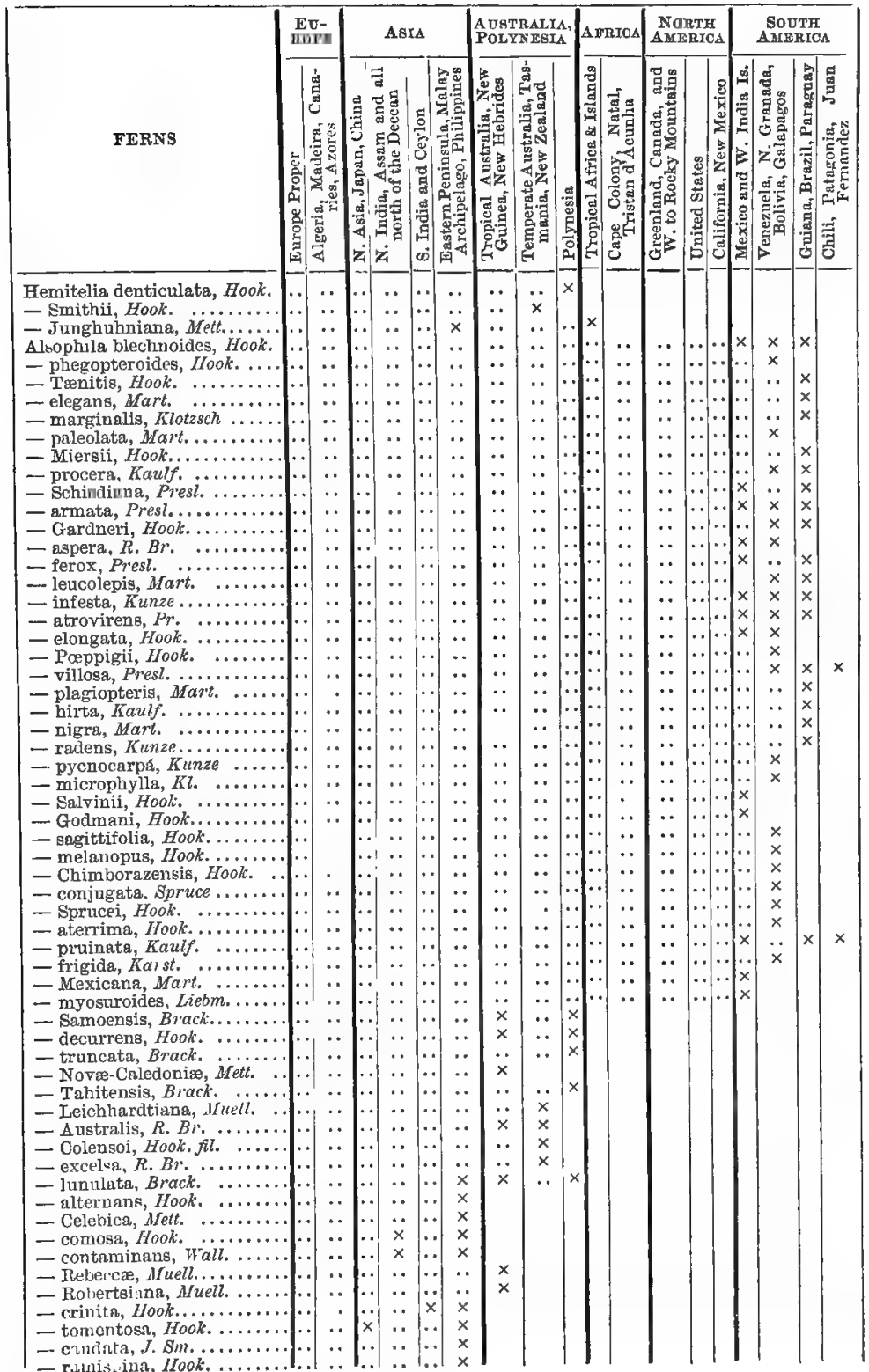




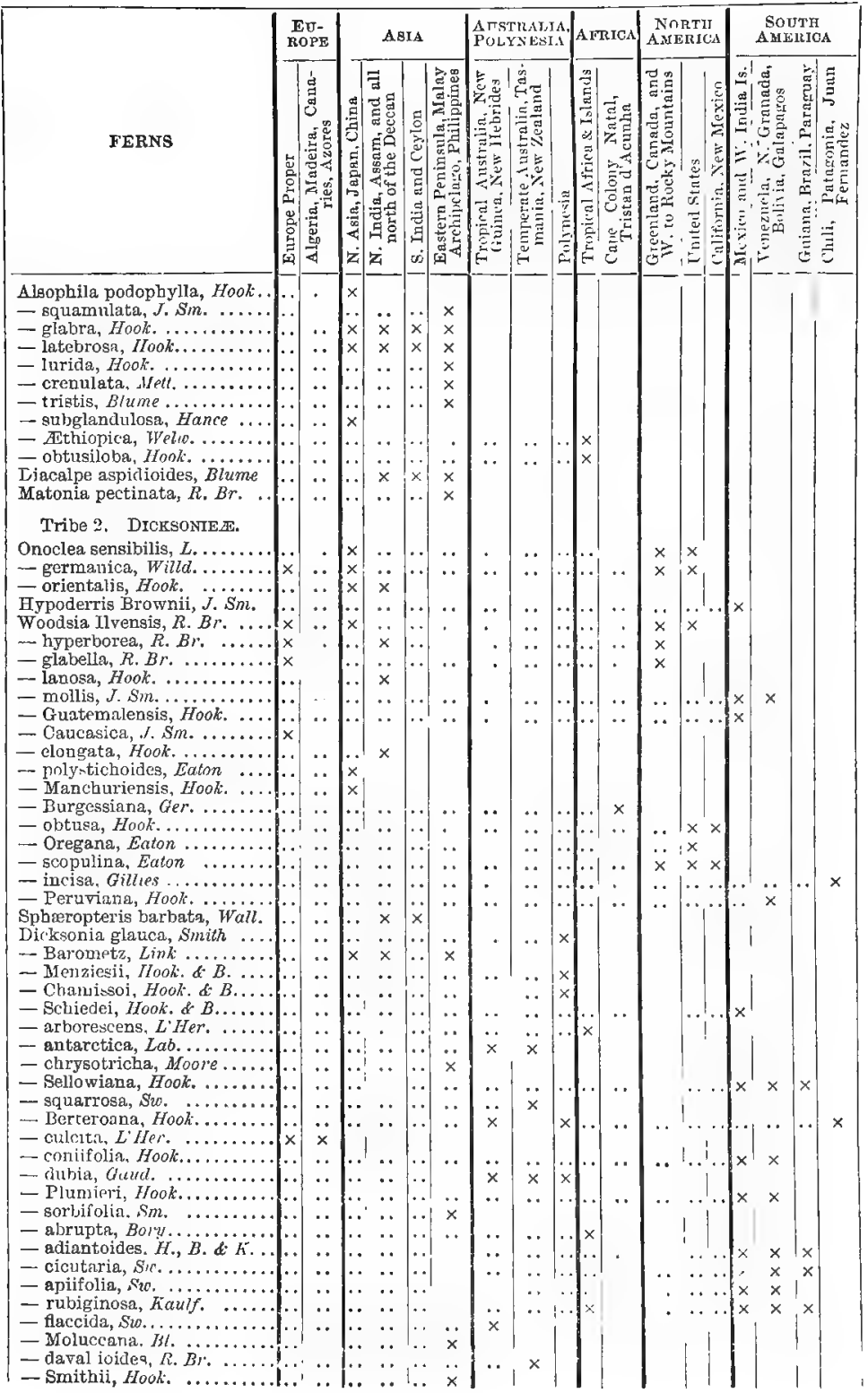




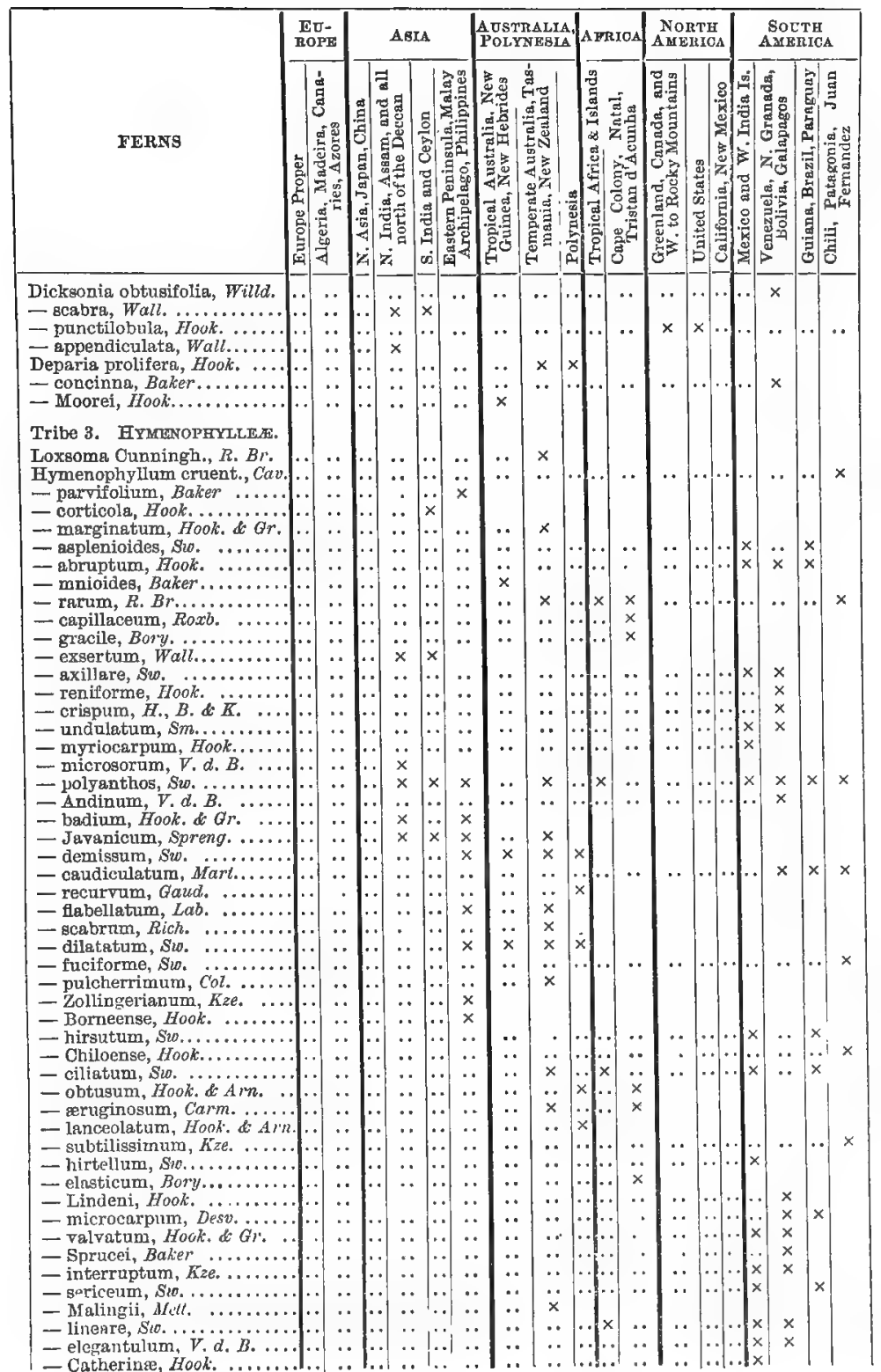




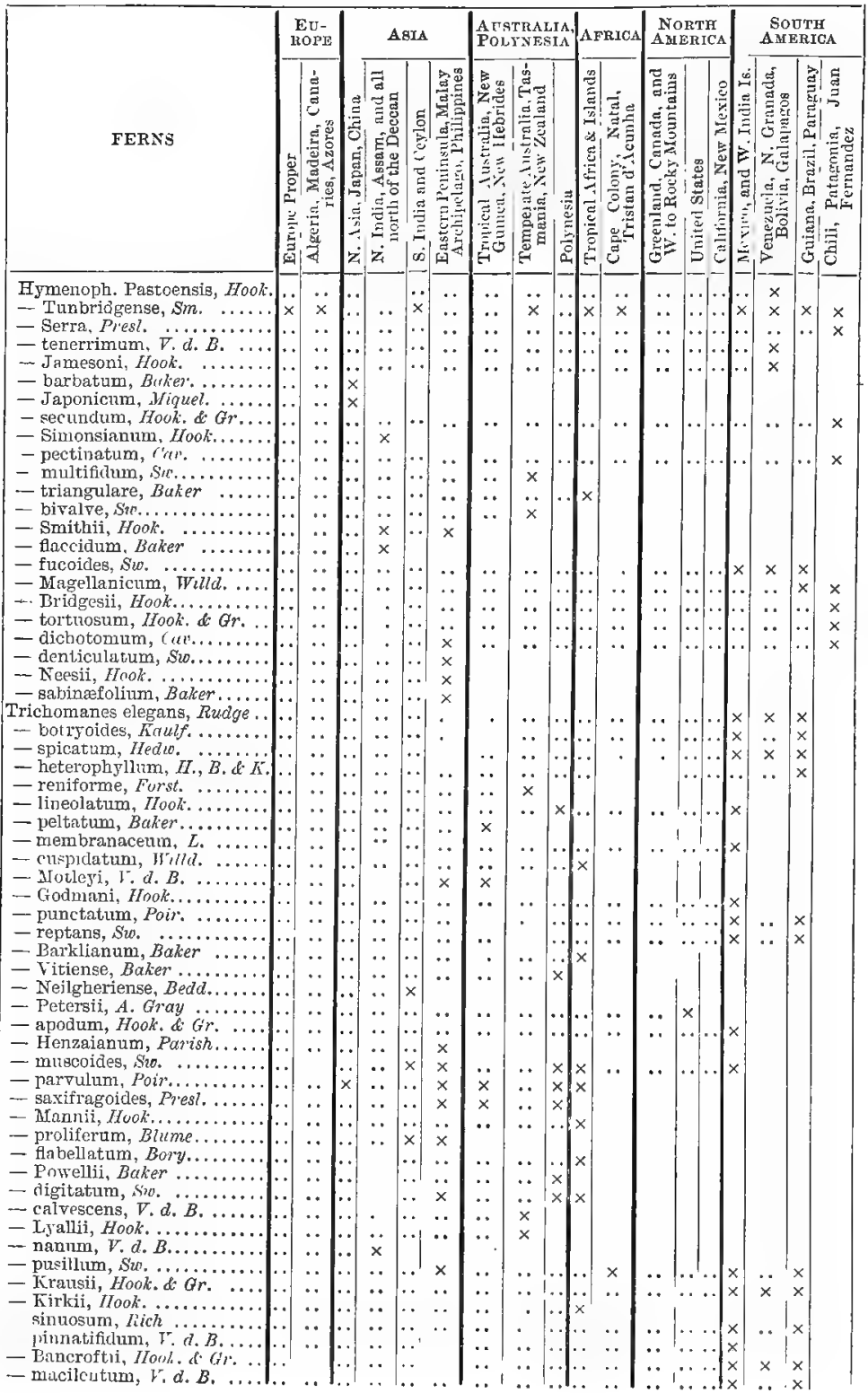




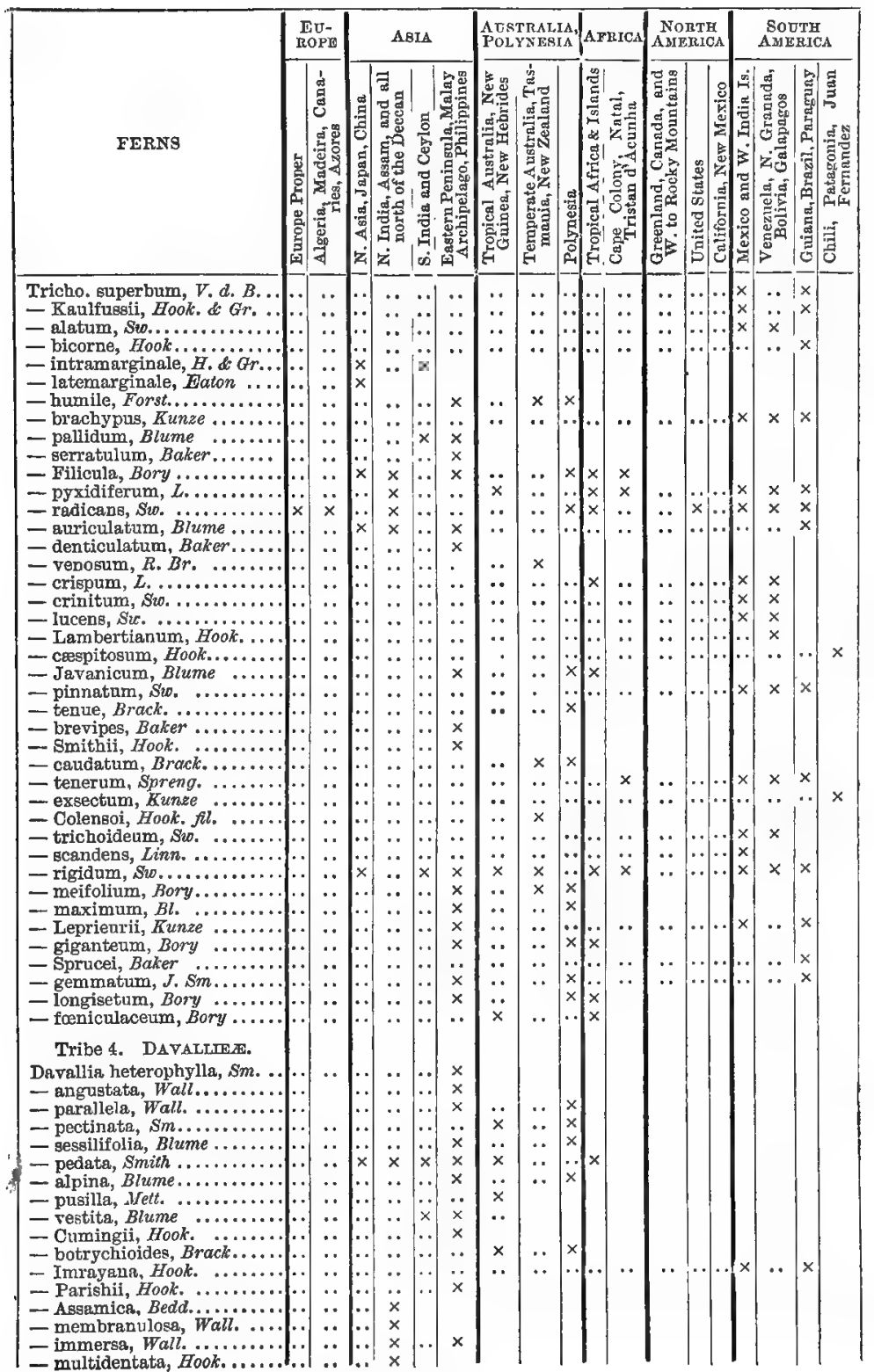




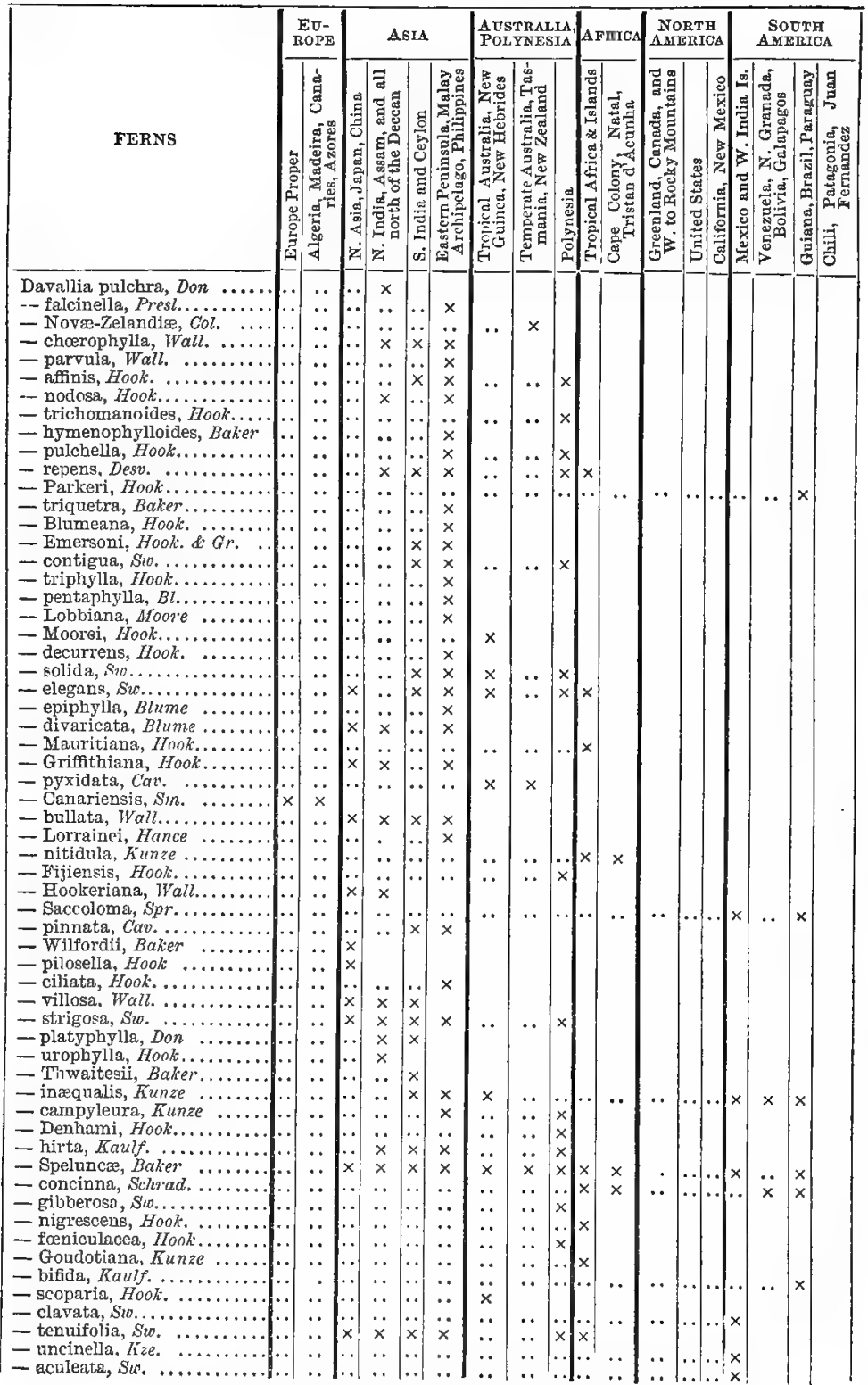




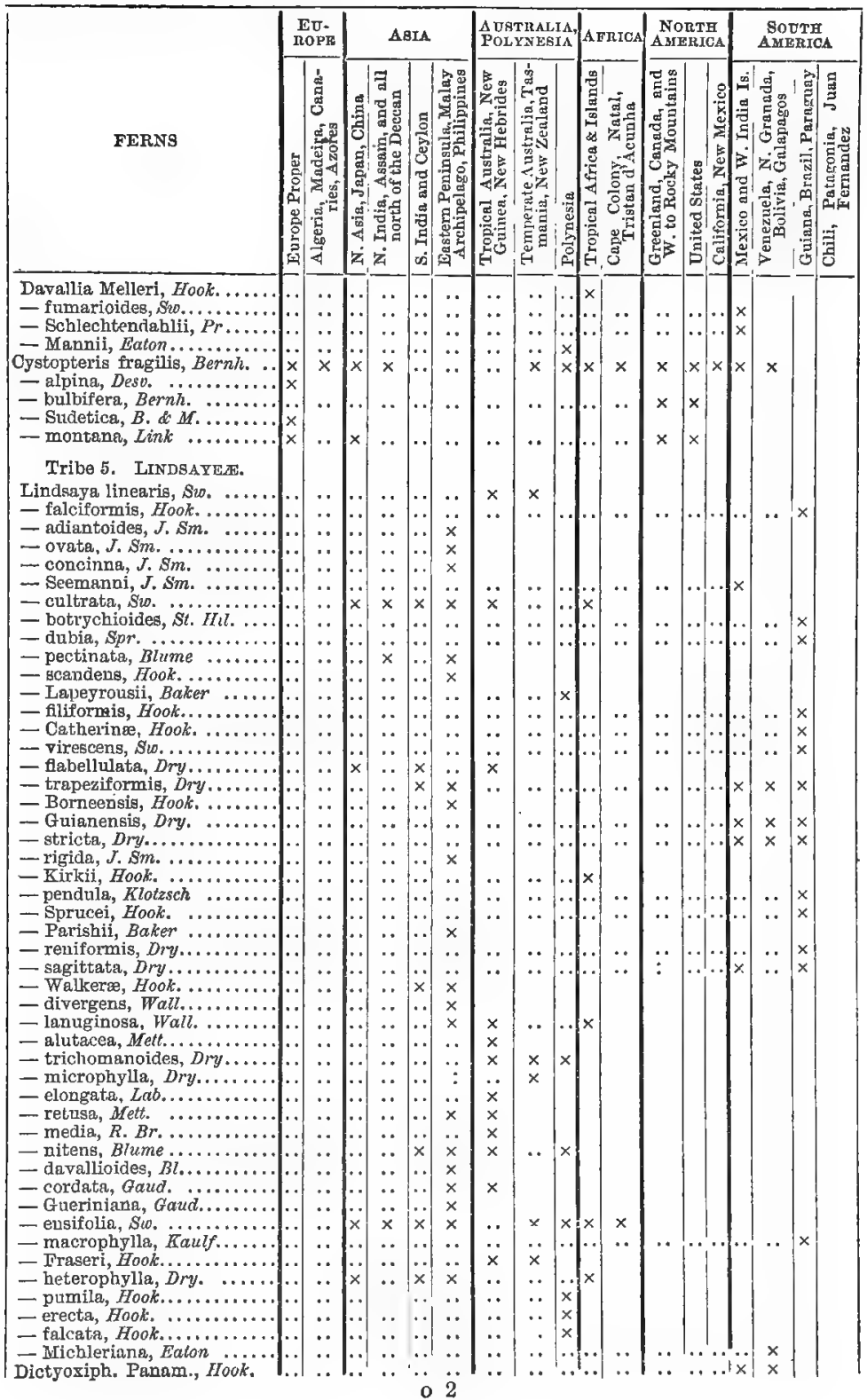




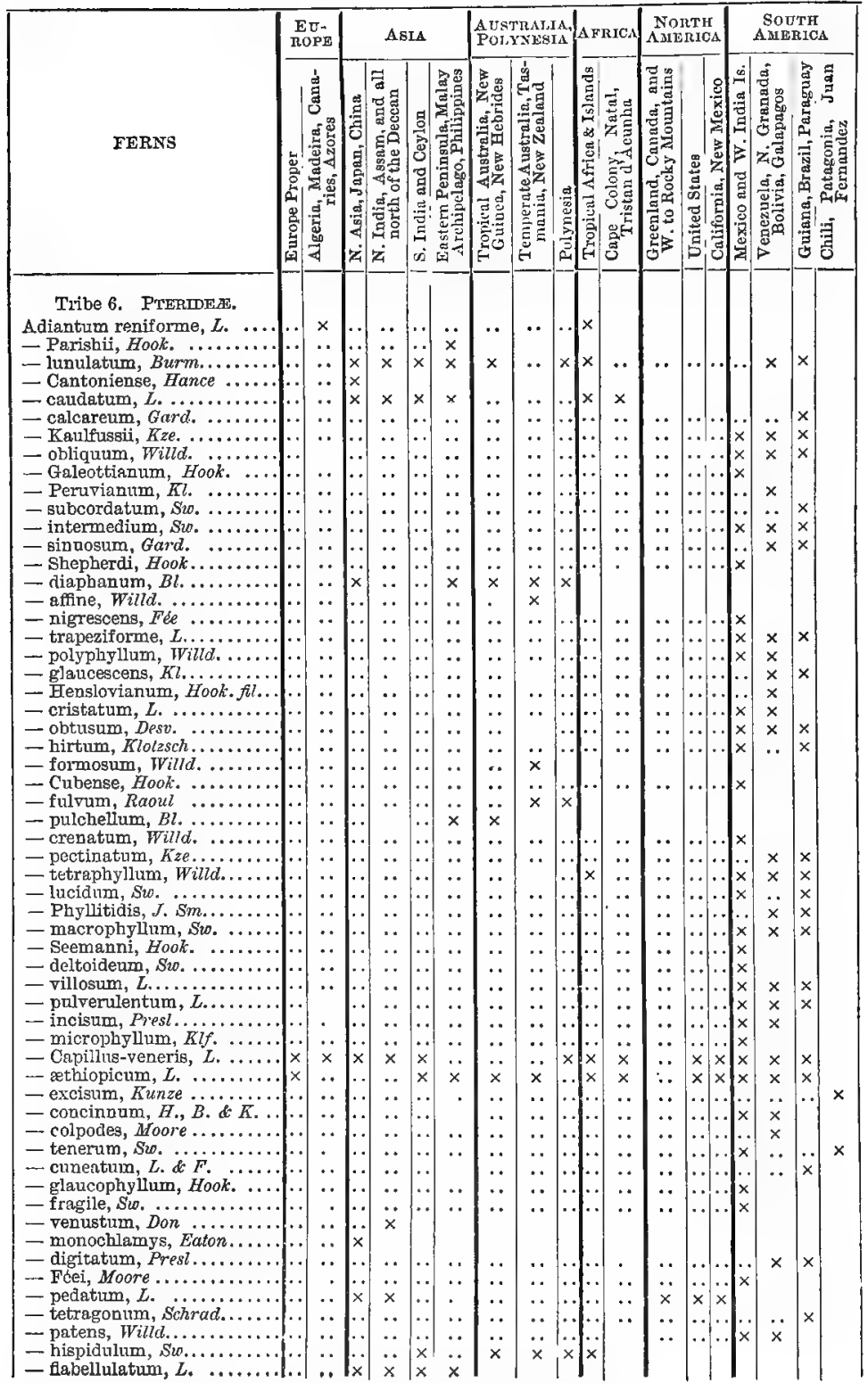




\begin{tabular}{|c|c|c|c|c|c|c|c|c|c|c|c|c|c|c|c|c|c|c|}
\hline \multirow[b]{2}{*}{ FERN3 } & \multicolumn{2}{|c|}{$\underset{\text { EOPE }}{\text { EU- }}$} & \multicolumn{4}{|c|}{ AsIA } & \multicolumn{3}{|c|}{ 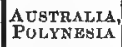 } & \multicolumn{2}{|c|}{ AFIOA } & \multicolumn{3}{|c|}{$\begin{array}{l}\text { Nor'r } \\
\text { AMERIOA }\end{array}$} & \multicolumn{4}{|c|}{$\begin{array}{l}\text { SOUTH } \\
\text { AMLRICA }\end{array}$} \\
\hline & 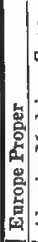 & 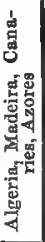 & 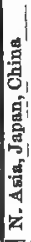 & 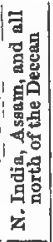 & 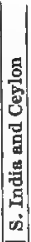 & 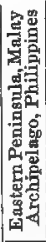 & 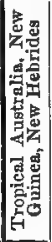 & 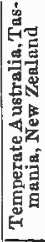 & 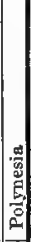 & 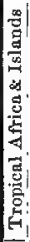 & 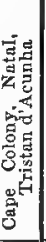 & 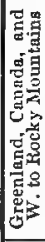 & 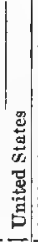 & 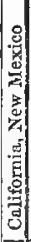 & 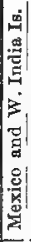 & 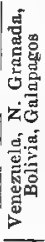 & 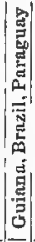 & 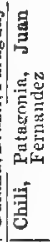 \\
\hline Adiantum Hewardia, Kunze & 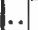 & $\because$ & & . & $\because$ & .. & 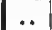 & 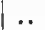 & $\cdots$ & $\cdots$ & . & $\because$ &.. & . . & $\ldots$ & .. & $x$ & \\
\hline - dolosum, Kunze ......... & $\therefore$ & $\because$ & 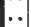 & $\ldots$ & $\ldots$ & . & $\cdots$ & . & .. & $\cdots$ & .. & .. & $\ldots$ & .. & $x$ & $x$ & $x$ & \\
\hline - olivaceum, Baker ........ & $\because$ & $\because$ & $\therefore$ & .. & $\therefore$ & $\ldots$ & $\cdots$ & .. & $\therefore$ & $\therefore$ & . & $\therefore$ &.. & $\because$ & {$\left[\begin{array}{l}n \\
\cdots\end{array}\right.$} & $\ldots$ & $x$ & \\
\hline - Leprieurii, Hook. . ... & $\therefore$ & .. & .. & $\therefore$ & $\therefore$ & $\cdots$ & $\therefore$ & . & . & $\because$ & . & -. & .. & $\cdots$ & $\cdots$ & . & $x$ & \\
\hline Ochropteris pallens, $J, S m$. . & $\ldots$ & .. & .. & . & .. & . & $\cdots$ & .. &.. & $x$ & & & & & & & & \\
\hline Lonchitis pubescens, Willd... & $\cdots$ & $\cdots$ & $\because$ & . &.. & .. & $\cdots$ & $\cdots$ & $\cdots$ & $x$ & $x$ & & & & & & & \\
\hline - occidentalis, Baker ...... & $\cdots$ & $\cdots$ & $\because$ & $\cdots$ & $\cdots$ & $\ddot{\sim}$ & $\because$ & $\ddot{q}$ & $\cdots$ & & & & & & & & & \\
\hline & $\because$ & $\because$ & $\because$ & 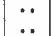 & $\because$. & $\begin{array}{l}x \\
\therefore\end{array}$ & $\begin{array}{l}x \\
\cdots\end{array}$ & $\begin{array}{l}x \\
x\end{array}$ & & & & & & & & & & \\
\hline - anthriscifolia, Presi....... & $\therefore$ & 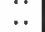 & $\therefore$ & $\because$ & $\because$ & $\therefore$ & $\because$ & . & $\cdots$ & $x$ & $x$ & & & & & & & \\
\hline - repens, Prest. ........... & $\ldots$ & $\cdots$ & $\ldots$ & . & $\ldots$ & .. & $\cdots$ & .. & .. & $\therefore$ & . & $\cdots$ & .. & .. & $x \mid$ & $x$ & $x$ & \\
\hline - hostilis, Presl........... & $\therefore$ & $\cdots$ & $\cdots$ & $\cdots$ & .. & .. & $\cdots$ & . & . & . & $\cdots$ & .. & .. & $\cdots$ & $x \mid$ & $\mathbf{x}$ & & \\
\hline - nigrescens, Hook....... & $\cdots$ & $\cdots$ & $\cdots$ & $\because$ & $\cdots$ & .. & $\cdots$ & $\cdots$ & $\because$ & $\cdots$ & $\cdots$ & -. & .. & $\ldots$ & $x$ & $\cdots$ & $\mathbf{x}$ & \\
\hline - Purdieana, Hook. ....... & $\cdots$ & $\cdots$ & $\cdots$ & . & $\cdots$ & $\cdots$ & $\cdots$ & . & $\ldots$ & $\cdots$ & $\cdots$ & .. & .. & .. & $x$ & $x$ & & \\
\hline - parallelogramma, Hook. .. & $\ldots$ & $\cdots$ & .. & : &.. & .. & $\cdots$ & $\because$ &.. & $\cdots$ & $\cdots$ & $\cdots$ & $\cdots$ & $\cdots$ & $\times$ & $x$ & & \\
\hline - Millefolium, Hook........ & $\cdots$ & *. & 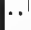 & . & $\cdots$ & . & $\cdots$ & $x$ & & & & & & & & & & \\
\hline $\begin{array}{l}\text { - Bergiana, Hook........... } \\
\text { - Californica, Hook. . . . . . }\end{array}$ & $\because$ & $\because$ & $\because$ & , & $\because$ & .. & $\ddot{0}$ & 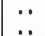 & $\because$ & $\begin{array}{l}x \\
\cdots\end{array}$ & $\begin{array}{l}x \\
\ldots\end{array}$ & .. & .. & $x$ & & & & \\
\hline Cheilanthes monticola, $\ddot{\text { Gar }} \dot{ }$. & $\because$ & $\because$ & $\ldots 1$ & $\ldots$ & $\because$ & $\because$ & $\because$ & .. & $\ldots$ & $\because$ & $\ddot{0}$ & $\ddot{*}$ & $\because$ & 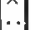 & $\cdots$ & $\cdots$ & $x$ & \\
\hline - Lindigii, Mett........... & .. & .. & $\therefore$ & $\ldots$ & $\therefore$ & $\therefore$ & .. & .. & .. & $\therefore$ & .. & $\ldots$ & $\ldots$ & $\therefore$ & $\cdots$ & $x$ & & \\
\hline - pteroides, Sw.......... & $\therefore$ & .. & $\therefore$ & $\ldots$ & $\ldots$ & $x$ & .. & $\therefore$ & $\cdots$ & $x$ & $x$ & & & & & & & \\
\hline - regularis, Mett. ......... & $\cdots$ & $\cdots$ & $\therefore$ & . & $\cdots$ & . & $\because$ & $\therefore$ & $\cdots$ & $\cdots$ & .. & $\cdots$ & ... & .. & $\because$ & . & $x$ & \\
\hline - paupercula, Mett. ....... & $\cdots$ & $\therefore$ & $\therefore$ & . & $\cdots$ & . & $\because$ & -. & $\ldots$ &.+1 & . & -. &.. & $\cdots$ & $x$ & & & \\
\hline 一 radiata, $R, B r . \ldots \ldots \ldots$ & $\cdots$ & $\cdots$ & $\cdots$ & . & $\ldots$ & . & . & .. & . & $\cdots$ & $\cdots$ & .. &.. & . & $\times$ & $x$ & $\times$ & \\
\hline - Capensis, Sw. . . . . . . . . & $\cdots$ & $\cdots$ & ... & . & $\cdots$ & . & .. & . & $\cdots$ & $\because$ & $x$ & & & & & & & \\
\hline - Kirkii, Hook. ........... & $\cdots$ & $\cdots$ & $\cdots$ & $\cdots$ & $\cdots$ & $\cdots$ & $\cdots$ & $\cdots$ & $\cdots$ & $x$ & & & & & & & & \\
\hline - pedata, $R . B r . \cdots \cdots \cdots$ & $\cdots$ & $\cdots$ & $\because$ & $\cdots$ & $\cdots$ & $\because$ & $\because$ & $\because$ & $\because$ & $\because$ & $\cdots$ & $\because$ & $\cdots$ & $\because$ & $\times$ & & & \\
\hline $\begin{array}{l}\text { - propinqua, Mett. .... } \\
\text { - dichotoma, Sio. ..... }\end{array}$ & $\because$ & $\because$ & $\cdots$ & $\because$ & $\cdots$ & $\because$ & .. & $\because$ & $\because$ & $\because$ & $\because$ & 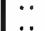 & $\because$ & $\because$ & $\because$ & $\begin{array}{l}x \\
x\end{array}$ & $x$ & \\
\hline & $\because$ & $\because$ & 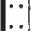 & $\because$ & $\because$. & $\because$ & .. & $\because$ & $\because$ & $\because$ & $\ddot{n}$ & $\ddot{\circ}$ & $\because$ & $\ddot{x} \mid$ & & & $\lambda$ & \\
\hline - Sohimperi, Hook. & $\cdots$ & $\because$ & .. & $\ldots$ & $\cdots$ & .. & .. & $\ldots$ & .. & $\times$ & & & & & & & & \\
\hline 一 incisa, Kunze ....... & .. & $\cdots$ & .. & $\ldots$ & $\ldots$ & . & $\cdots$ & . & .. & $\cdots$ & - & & $\cdots$ & $\ldots$ & $\cdots$ & .. & $x$ & \\
\hline — chlorophyla, $S w . \ldots \ldots \ldots$ & $\cdots$ & $\cdots$ & .. & $\ldots$ & .. & .. & $\cdots$ & $\cdots$ &.. & $\cdots$ & . & • & $\cdots$ & .. & $\cdots$ & $\because$ & $x$ & \\
\hline - micropteris, Swo......... & $\cdots$ & $\ldots$ & $\therefore$ & . & .. & -. & 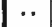 & . & $\cdots$ & $\cdots$ & $\cdots$ & $\cdots$ & $\cdots$ & $\cdots$ & 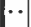 & $x$ & & \\
\hline - Matthewsif, Kunze „.... & $\ddot{x}$ & $\ddot{x}$ & $\ddot{x}$ & $\ddot{x}$ & .. & -・ & $\because$ & $\because$ & .. & . & $\cdots$ & $\cdots$ & .. & $\cdots$ & $\cdots$ & $x$ & & \\
\hline - fragrans, $W . \& B \ldots \ldots \ldots$ & $x$ & $x$ & $x$ & $x$ & & & & & & & & & & & & & & \\
\hline $\begin{array}{l}\text { - arabica, Dec. ........ } \\
\text { - vestita, Sw......... }\end{array}$ & $\because$ & 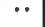 & $\because$ & 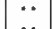 & $\cdots$ & $\because$ & - & $\because$ & $\because$ & {$\left[\begin{array}{l}x \\
\cdots\end{array}\right.$} & & • & $x$ & & & & & \\
\hline - pilosa, Goldm......... & $\because$ & $\because$ & $\because$ & 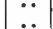 & $\because$ & $\because$ & $\ddot{*}$ & $\ddot{*}$ & $\because$ & $\because$ & $\because$ & $\ddot{0}$ & .. & .. & $\because$ & $x$ & & \\
\hline - microphylla, swo. ... & ... & $\ldots$ & $\therefore$ & $\cdots$ & I.. & .. & $\cdots$ & $\cdots$ & $\ldots$ & & . & .. &.. & $x$ & $x$ & $x$ & & \\
\hline - Mysurensis, Wall. .. & $\ldots$ & $\ldots$ & $x$ & . & $x$ & & & & & & & & & & & & & \\
\hline - fragilis, Hook....... & .. & $\cdots$ & . . & $\cdots$ & $\ldots$ & $x$ & & & & & & & & & & & & \\
\hline - hirt $\Omega$, Sw.......... & $\ldots$ & .. & ... & . & .. & $\cdots$ & - & . & .. & $x$ & $x$ & & & & & & & \\
\hline - viscosa, Kaulf. ..... & .. & $\cdots$ & .. & . & ... & .. & $\cdots$ & $\cdots$ & .. & $\cdot$ & $\cdots$ & .. & .. & $x$ & $\bullet$ & $x$ & & \\
\hline 一 Hispanica, Mett..... & $x$ & & & & & & & & & & & & & & & & & \\
\hline - pulchella, Bory .... & $\cdots$ & $x$ & & & & & & & & & & & & & & & & \\
\hline 一 varians, Hook........ & $\cdots$ & $\cdots$ & $x$ & $x$ & $x$ & $x$ & & & & & & & & & & & & \\
\hline - subvillosa, Hook... . & $\because$ & .. & $\cdots$ & $x$ & & & & & & & & & & & & & & \\
\hline - Dalhousia, Hook. ... & $\cdots$ & $\cdots$ & $\cdots$ & $x$ & $x$ & & & & & & & & & & & & & \\
\hline - allosuroides, Ifet. . & $\cdots$ & $\cdots$ & $\cdots$ & $\cdots$ & .. & $\cdots$ & $\ddot{v}$ & $\ddot{x}$ & $\cdot$ & $\cdots$ & $\because$ & $\cdots$ & $\cdots$ & $\cdots$ & $x$ & & & \\
\hline - Sieberi, Kunze..... & $\cdots$ & $\cdot$ & $\because$ & $\cdots$ & 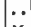 & 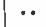 & $x$ & $x$ & & & & & & & & & & \\
\hline - bullosa, Funze ... & $\because$ & . & $\cdots$ & 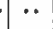 & $x$ & & & & & $x$ & $x$ & & & & & & & \\
\hline - multifida, Sto...... & & . & $\because$ & 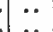 & $\cdots$ & $x$ & $\because$ & 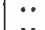 & $\because$ & & .. & . & -1 & $x$ & & & & \\
\hline $\begin{array}{l}\text { - Wrightii, Hook.... } \\
\text { - tenuifolia, Sw..... }\end{array}$ & . & $\ddot{*}$ & $\ddot{x}$ & $\ddot{x}$ & $\ddot{x}$ & $\ddot{x}$ & -. & $\ddot{x}$ & & & & & & & & & & \\
\hline - flexuosa, Kunze ........ & $\cdots$ & .. & . & $\cdots$ & .. & $\cdots$ & . & $\cdots$ & * & 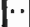 & $\ddot{q}$ & $\cdots$ & $\cdots$ & $\cdot$ & $\because$ & $\because$ & $x$ & \\
\hline - induta, Kunze .. & & .. & . & $\cdots$ & .. & $\cdots$ & . & $\cdots$ & & & $x$ & & & & & & & \\
\hline - lanuginosa, Nutt.... & $\cdots$ & '. & $\because$ & $\because$ & $\cdots$ & $\because$ & $\cdots$ & $\cdots$ & & & $\cdots$ & $\cdots$ & $\times$ & $\times$ & & & & \\
\hline $\begin{array}{l}\text { - Szovitzii, } F \text {. \& } M \ldots \ldots \\
\text { - gracillima, Eaton } \ldots . .\end{array}$ & $x$ & & $x$ & $\begin{array}{l}x \\
\ldots\end{array}$ & & & & & & & & & & & & & & \\
\hline
\end{tabular}




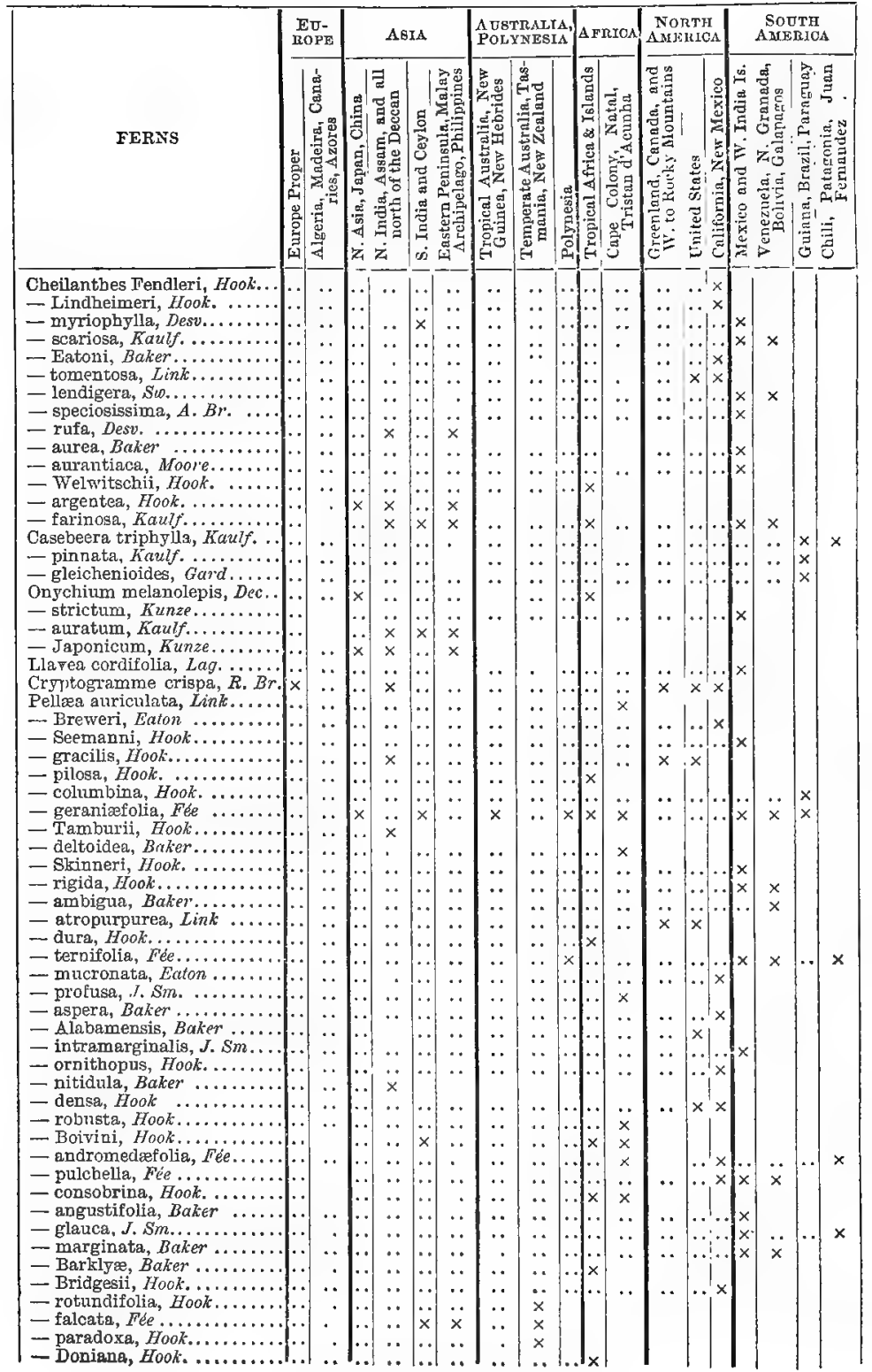




\begin{tabular}{|c|c|c|c|c|c|c|c|c|c|c|c|c|c|c|c|c|c|c|}
\hline \multirow[b]{2}{*}{ FERNS } & \multicolumn{2}{|c|}{$\underset{\text { ROPE }}{\text { EU- }}$} & \multicolumn{4}{|c|}{ AsIA } & \multicolumn{3}{|c|}{$\begin{array}{l}\text { A USTTR } \triangle L I A, \\
\text { POLYNESA }\end{array}$} & \multicolumn{2}{|c|}{ ATRTOA } & \multicolumn{3}{|c|}{$\begin{array}{l}\text { NonTII } \\
\text { AMERICA }\end{array}$} & \multicolumn{4}{|c|}{$\begin{array}{c}\text { SotTh } \\
\text { AMERICA }\end{array}$} \\
\hline & 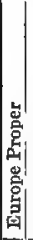 & 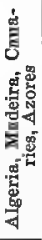 & 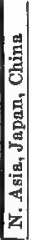 & 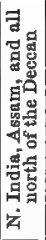 & & 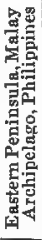 & 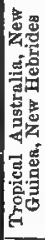 & 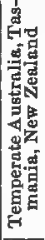 & . 영 & 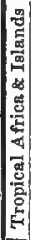 & 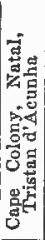 & 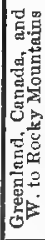 & 兽 & 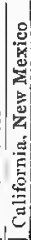 & 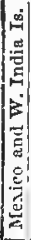 & 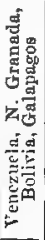 & 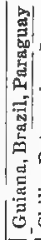 & 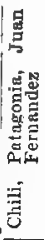 \\
\hline Pellæa hastata, Link & & $\cdots$ & $\cdots$ & $\cdots$ & $\cdots$ & . & • & & . & $x$ & $\mathbb{x}$ & & & & & & & \\
\hline - calomelanos, Link . & $\therefore$ & . & $\therefore$ & $\ddot{x}$ & $\because$ & $\because$ & .. & $\therefore$ & . & $\hat{x}$ & a & & & & & & & \\
\hline - cordeta, J. Sm..... & $\therefore$ & $\therefore$ & $\therefore$ & $\because$ & $\cdots$ & $\therefore$ & $\therefore$ & .. & $\cdots$ & $\cdots$ & $\cdots$ & $\cdots$ & $\cdots$ & $x$ & $x$ & $x$ & & \\
\hline keana, Baker .. & $\therefore$ & $\therefore$ & .. & $\because$ & $\cdots$ & $\therefore$ & $\therefore$ & $\ldots$ & . & $\because$ & $x$ & . & & & & & & \\
\hline iculata, Baker ...... &.. & .. & $\therefore$ & .. & $\because$ & . & . & .. & . & $\times$ & & & & & & & & \\
\hline Pteris longifolia, $L ., \ldots \ldots$ & $x$ & $x$ & $x$ & $x$ & $x$ & $x$ & $x$ & $x$ & $x$ & $x$ & $x$ & $\because$ & $x$ & & $x$ & $x$ & & \\
\hline $\begin{array}{l}\text { Caud, Blume........ } \\
J . S m . \ldots \ldots \ldots \ldots\end{array}$ & $\because$ & $\because$ & $\because$ & $\because$ & $\cdots$ & $x$ & & & & & & & & & & & & \\
\hline - Cretica, $L, \ldots \ldots \ldots$ & $\ddot{x}$ & $\because$ & $\ddot{x}$ & $\ddot{x}$ & $\ddot{x}$ & $\begin{array}{l}x \\
x\end{array}$ & & & & & & & & & & & & \\
\hline cida, Presl..... & . & $\because$ & 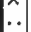 & $\begin{array}{l}x \\
x\end{array}$ & $\left(\begin{array}{l}x \\
x\end{array}\right.$ & $\begin{array}{l}x \\
x\end{array}$ & $\because$ & $\because$ & $\mid \begin{array}{l}x \\
\cdots\end{array}$ & $\begin{array}{l}x \\
x\end{array}$ & $x$ & $\cdots$ & $x$ & & $x \mid$ & & & \\
\hline - Hookeriana, $A g$. .. & .. & $\ldots$ & .. & $\ldots$ & $x$ & & & & & & & & & & & & & \\
\hline - dactylina, Hook̆. . & $\cdots$ & .. & .. & $x$ & & & & & & & & & & & & & & \\
\hline - umbrosa, R. Br.......... & .. & .. & .. & . & $\cdots$ & ". & $\cdots$ & $x$ & & & & & & & & & & \\
\hline - serrulata, L. fil.......... & $\ldots$ & 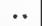 & $x$ & & & & & & & & & & & & & & & \\
\hline - crenate, Sho. ...... & $\cdots$ & $\therefore$ & $x$ & $x$ & $x$ & * & $x$ & $\cdots$ & $x$ & & & & & & & & & \\
\hline , J.Sm,,$\ldots \ldots \ldots$ & . & $\cdots$ & $\therefore$ & $\because$ & $\cdots$ & $x$ & & & & & & & & & & & & \\
\hline $\begin{array}{l}\text { morpha, Fée....... } \\
\text { thii, Hook.......... }\end{array}$ & $\therefore$ & -. & $\therefore$ & $\cdots$ & $\cdots$ & $x$ & & & & & & & & & & & & \\
\hline $\begin{array}{l}\text { - Griflithii, Hook..... } \\
\text { - Madagascarioa, } \mathrm{Ag} \text {. }\end{array}$ & $\cdot$. & $\cdots$ & 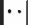 & $x$ & & & & & & & & & & & & & & \\
\hline $\begin{array}{l}\text { - Madagascarica, } A g \text {. } \\
\text { - mutilata, } L . \ldots . . . \text {. }\end{array}$ & $\cdots$ & $\cdot \cdot$ & $\because$ & $\cdots$ & $\cdots$ & . & $\because$ & $\cdots$ & $\cdot \cdot$ & $x$ & & & & & & & & \\
\hline - semipinnata, $\not L \ldots \ldots$ & $\cdot$ & $\cdots$ & $\ddot{x}$ & $\ddot{x}$ & $\because \ddot{x}$ & $\because$ & $\cdots$ & $\cdots$ & $\cdots$ & $\cdots$ & $\cdots$ & - & $\because$ & $\cdots$ & $\times$ & & & \\
\hline - paucinervata, $F \ddot{e} e$. & $\because$ & $\because$ & $x$ & $x$ & $x$ & $x$ & & & & & & & & & & & & \\
\hline - litobrochioides, $K l$. & $\because$ & $\because$ & $\because$ & $\because$ & $\because$ & $\because$ & $\because$ & $\because$ & $\because$ & $\because$ & $\because$ & $\because$ & $\because$ & . & $x$ & & & \\
\hline tiæfolja, Hook. & .. & .. & $\ldots$ & $\ldots$ & $\cdots$ & $\therefore$ & $\therefore$ & $\therefore$ & . & $\because$ & $\cdots$ & $\ldots$ & $\ldots$ & & $\because$ & $\because$ & $x$ & \\
\hline - Dalhousiæ, Hook. .... & .. & $\therefore$ & .. & $\cdots$ & $\therefore$ & $x$ & & & & & & & & & $\cdots$ & $\cdots$ & $\cdots$ & $x$ \\
\hline - irregularis, Kaulf. ... & .. & .. & .. & .. & $\ldots$ & . & $\cdots$ & $\cdots$ & $x$ & & & & & & & & & \\
\hline ta, Retz. & .. & .. & $x$ & $x$ & $x$ & $x$ & $x$ & .. & .. & $\times$ & $x$ & $\cdots$ &.. & .. & $x$ & $x$ & $x$ & \\
\hline pinnula, Wall. & ... & . & $x$ & $x$ & $x$ & $x$ & & & & & & & & & & & & \\
\hline sa, Gaud. ... & . & $\cdots$ & $\ldots$ & $x$ & $\ldots$ & $x$ & *. & $\cdots$ & $\times$ & & & & & & & & & \\
\hline - pungens, Willd. . & $\cdots$ & $\cdots$ & $\therefore$ & $\cdots$ & $\ldots$ & $\cdots$ & 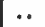 & $\cdots$ & . & $\cdots$ & $\because$ & $\cdots$ & 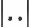 & $\cdots$ & $x$ & *. & $x$ & \\
\hline - scabra, Bory. & . . & $\cdots$ & ... & ." & $\cdots$ & $\cdots$ & $\cdots$ & . & .. & $x$ & & & & & & & & \\
\hline a, Roxb. & .. & $\cdots$ & ... & $\cdots$ & $\ldots$ & . & $\cdots$ & $\cdots$ & . & $x$ & & & & & & & & \\
\hline aledonise, Hook & . & .. & .. & - & .. & $\cdots$ & $x$ & & & & & & & & & & & \\
\hline phylla, $L . \ldots$. & .. & .. & .. & .. & $\cdots$ & .. & $\cdots$ & $\cdots$ & . & $\cdots$ & $\cdots$ & - &.. & $\cdots$ & $x$ & . & $x$ & \\
\hline - gracilis, Fee..... & . & . & $\ldots$ & $\cdot$ & $\cdots$ & . & $\cdots$ & $\cdots$ & . & $\therefore$ & $\cdots$ & .. & $\therefore$ & $\therefore$ & $\therefore$ & 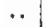 & $x$ & \\
\hline - laciniata, Willd,...... &.. & $\cdots$ & .. & $\cdots$ & $\cdots$ & $\cdots$ & $\cdots$ & .. & .. & $\because$ & . & $\cdots$ & $\ldots$ & .. & $x$ & & & \\
\hline - arguta, Ait......... & $x$ & $x$ & $\cdots$ & .. & $\cdots$ & $\cdots$ & • & $\cdots$ & - & $x$ & & & & & & & & \\
\hline - flabellata, Thunb. . & & .. & $\cdots$ & .. & $\cdots$ & $\cdots$ & $\cdots$ & $\cdots$ & .. & $x$ & $x$ & & & & & & & \\
\hline - tremula, $R . B r .$. & 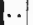 & * & $\cdots$ & $\cdots$ & $\cdots$ & $\cdots$ & & $x$ & & & & & & & & & & \\
\hline sis, Desv. & ... & .. & $\cdots$ & $\cdots$ & .. & $\cdots$ & $\cdots$ & $\because$ & $\cdots$ & $\ldots$ & $\cdots$ & $\cdots$ & $\ldots$ & . & * & . & $\cdots$ & $x$ \\
\hline ens, Ag.... & ..) & $\cdots$ & .. & $x$ & $x$ & & & & & & & & & & & & & \\
\hline 一 deflexs, Link .... & $\cdots$ & $\cdots$ & . & $\cdots$ & $\cdots$ & $\cdots$ & - & $\cdots$ & . & $\cdots$ & $\cdots$ & $\cdots$ & $\cdots$ & $\cdots$ & $x$ & $\because$ & $x$ & \\
\hline - coriacea, Dest. & 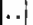 & .. & & - & $\cdots$ & $\cdots$ & - & $\cdots$ & . & . & .. & $\cdots$ &.. & $\cdots$ & $\therefore$ & $x$ & & \\
\hline - brevisora, Baker. . & .. & . &. & $\because$ & .. & . & $\because$ & $\cdots$ & . . & $x$ & & & & & & & & \\
\hline - aquilina, $L . \ldots$ & $\times$ & $x$ & $x$ & $x$ & $x$ & $x$ & $x$ & $x$ & $x$ & $x$ & $x$ & $\cdots$ & $x$ & $x$ & $x$ & $x$ & $x$ & \\
\hline a, Baker... & . & $\cdots$ & $\ldots$ & $\cdots$ & $\cdots$ & $\cdots$ & $\cdots$ & 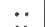 & .. & $\cdots$ & $\cdots$ & $\cdots$ & $\cdots$ & $\cdots$ & $x$ & $x$ & & \\
\hline — scaberula, Rich. & & .. & & - & $\ldots$ & .. & & $\mathrm{x}$ & & & & & & & & & & \\
\hline - rugulosa, Lab. & & . & .. & $\cdots$ & $\ldots$ & $\ldots$ & $x$ & *. & $x$ & & & & & & & & & \\
\hline - lomariacea, Kunze & . & $\cdots$ & $\cdots$ & . & $\ldots$ & . & $\cdots$ & $\cdots$ & . & $\cdots$ & - & $\cdots$ & . & & . & $\cdots$ & $x$ & \\
\hline - grandifolia, $L$. . & & $\cdots$ &.. & : & $\cdots$ & $\cdots$ & $\cdots$ & $\ldots$ & .. & $\cdots$ & $\cdots$ & $\cdots$ & $\ldots$ & & $x$ & & & \\
\hline 一 biaurita, $L, \ldots \ldots$ & .. & $\cdots$ & $x$ & $x$ & $x$ & $x$ & .. & .. & . & $x$ & $\cdots$ & .. & $\cdots$ & .. & $x$ & .. & $x$ & \\
\hline tens, Hook. & & ・ & .. & .. & $x$ & $x$ & $\cdots$ & .. & $x$ & & & & & & & & & \\
\hline - triplicata, $A g .$. & .. & $\cdots$ & .. & $\because$ & $\cdots$ & - & $\cdots$ & $\cdots$ & . & $x$ & & & & & & & & \\
\hline - Melleri, Baker... & & $\cdots$ & $\cdots$ & .. & $\ldots$ & $\cdots$ & .. & $\cdots$ & . & $x$ & & & & & & & & \\
\hline - Wallichiana, $\boldsymbol{A} g$. & & . & : & $x$ & $\cdots$ & $x$ & & & & & & & & & & & & \\
\hline - lonchophora, Mett.. & & .. & $\cdots$ & $\cdots$ & .. & .. & *. & $\cdots$ & .. & .. & .. & .. & .. & - & $\cdots$ & .. & $x$ & \\
\hline ittifolia, Raddi. & & . & & . & $\cdots$ & * & .. & $\cdots$ & .. & 1 & -. & $\cdots$ & $\cdots$ & $\cdots$ & $\because \cdot$ & .. & $x$ & \\
\hline ens, Wall. & & . & $\ldots$ & $\ldots$ &. & $x$ & & & & & & & & & & & & \\
\hline hopus, Mell. . & & $\cdots$ & . & . & $\because$ & .. & - & $\cdots$ & . & & $\cdots$ & -. & $\cdots$ & & $\because$ & $\ddot{x}$ & $x$ & \\
\hline$-p$ & & & & & & & & & & & & & & & & $x$ & & \\
\hline
\end{tabular}




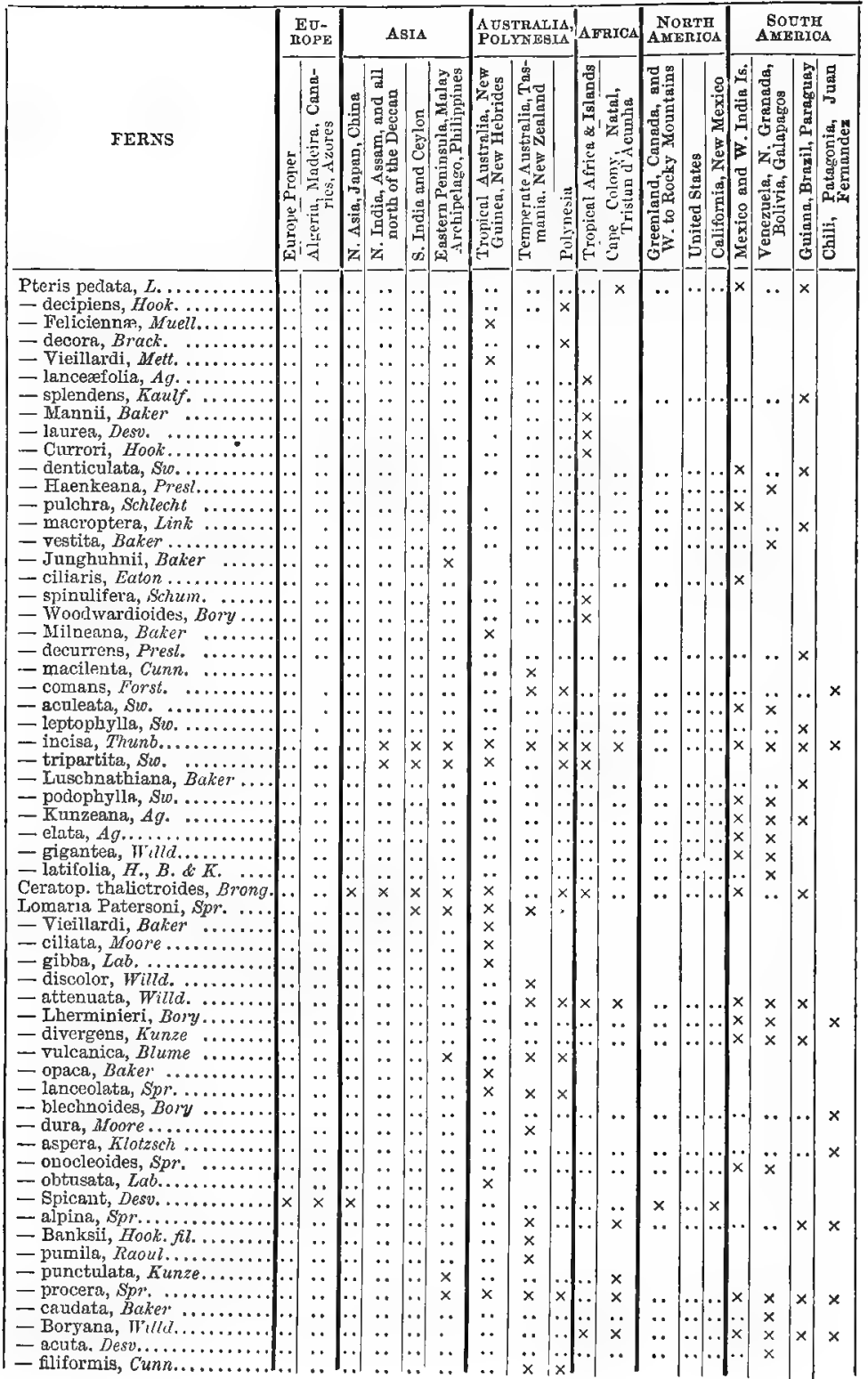




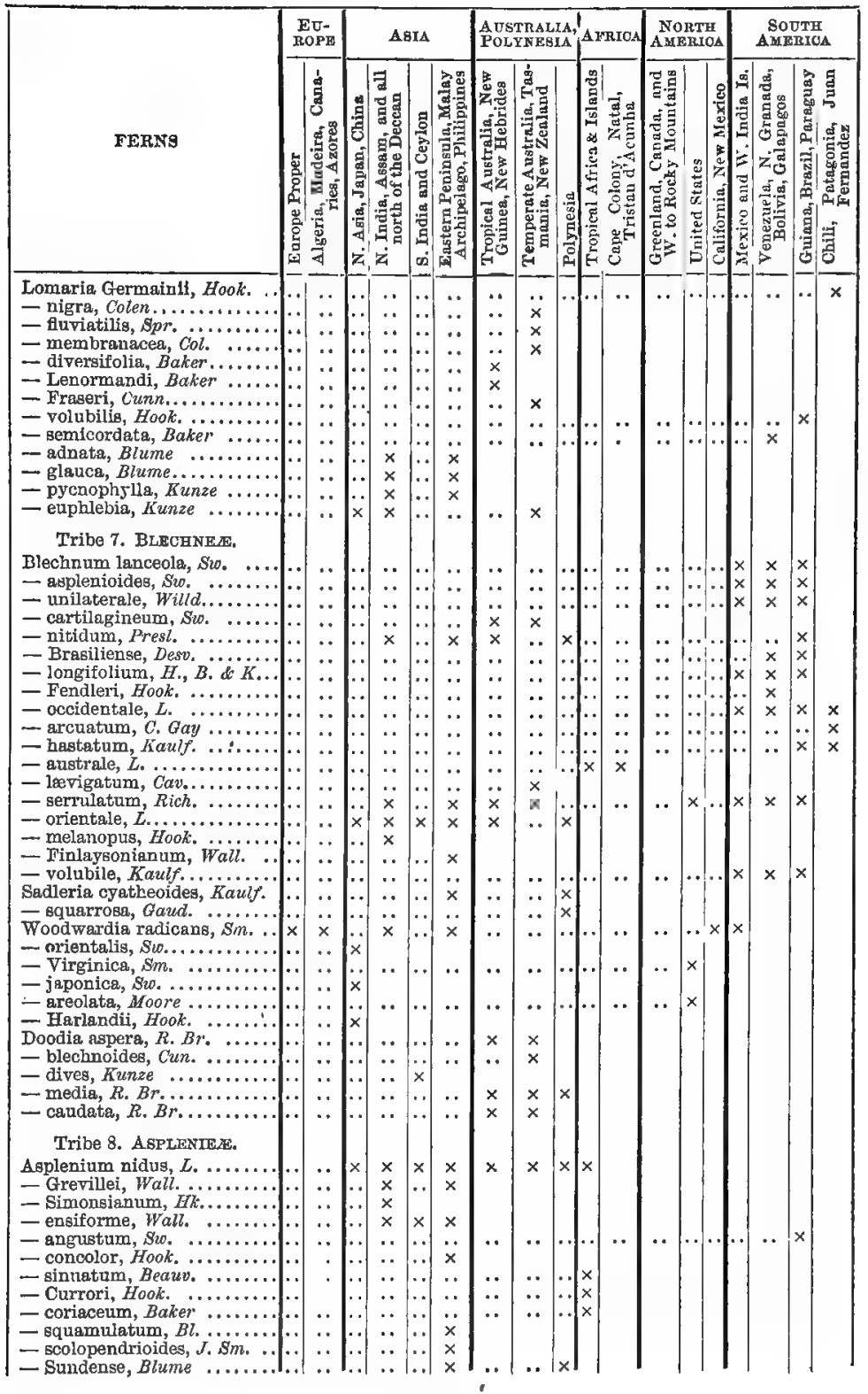




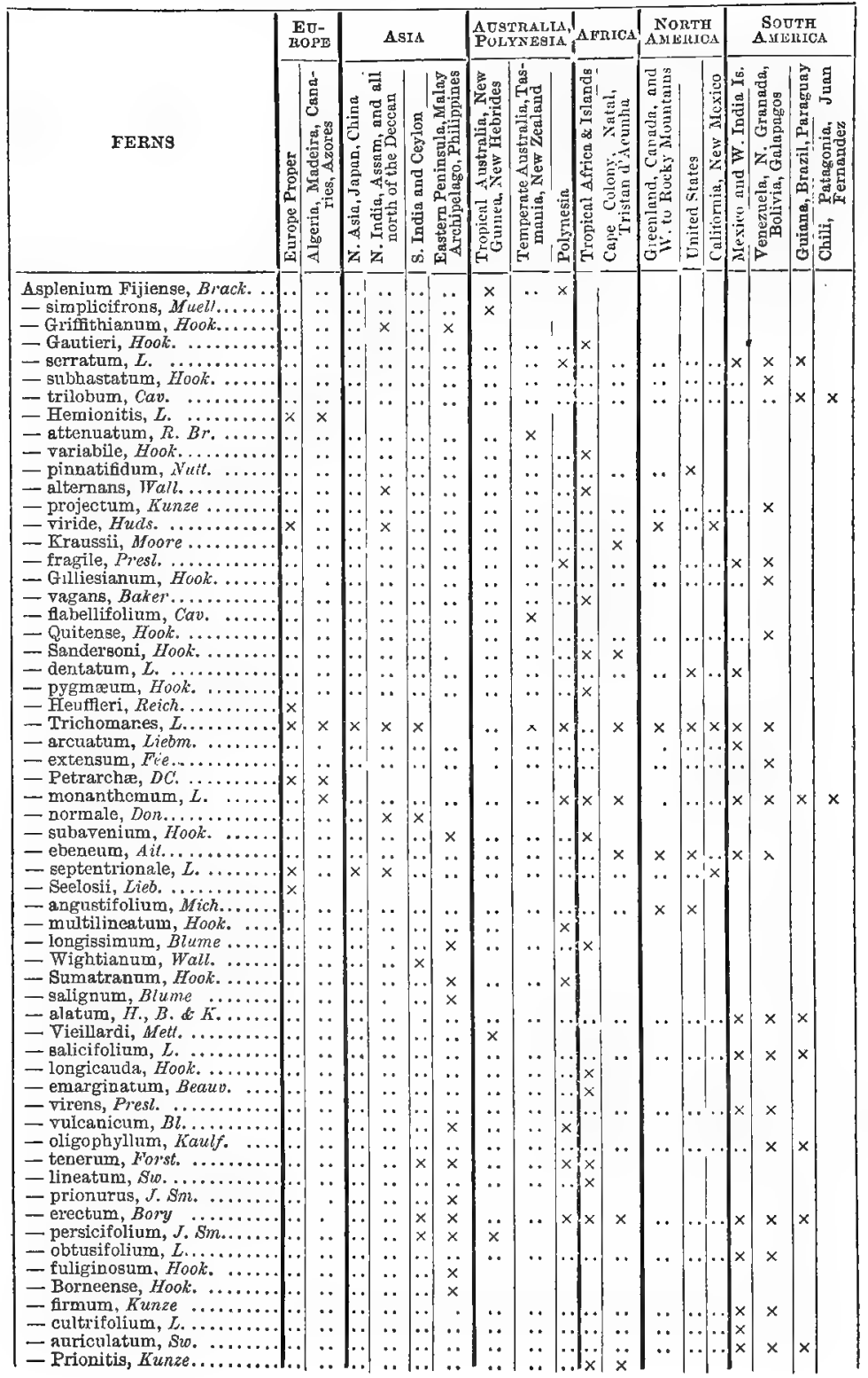




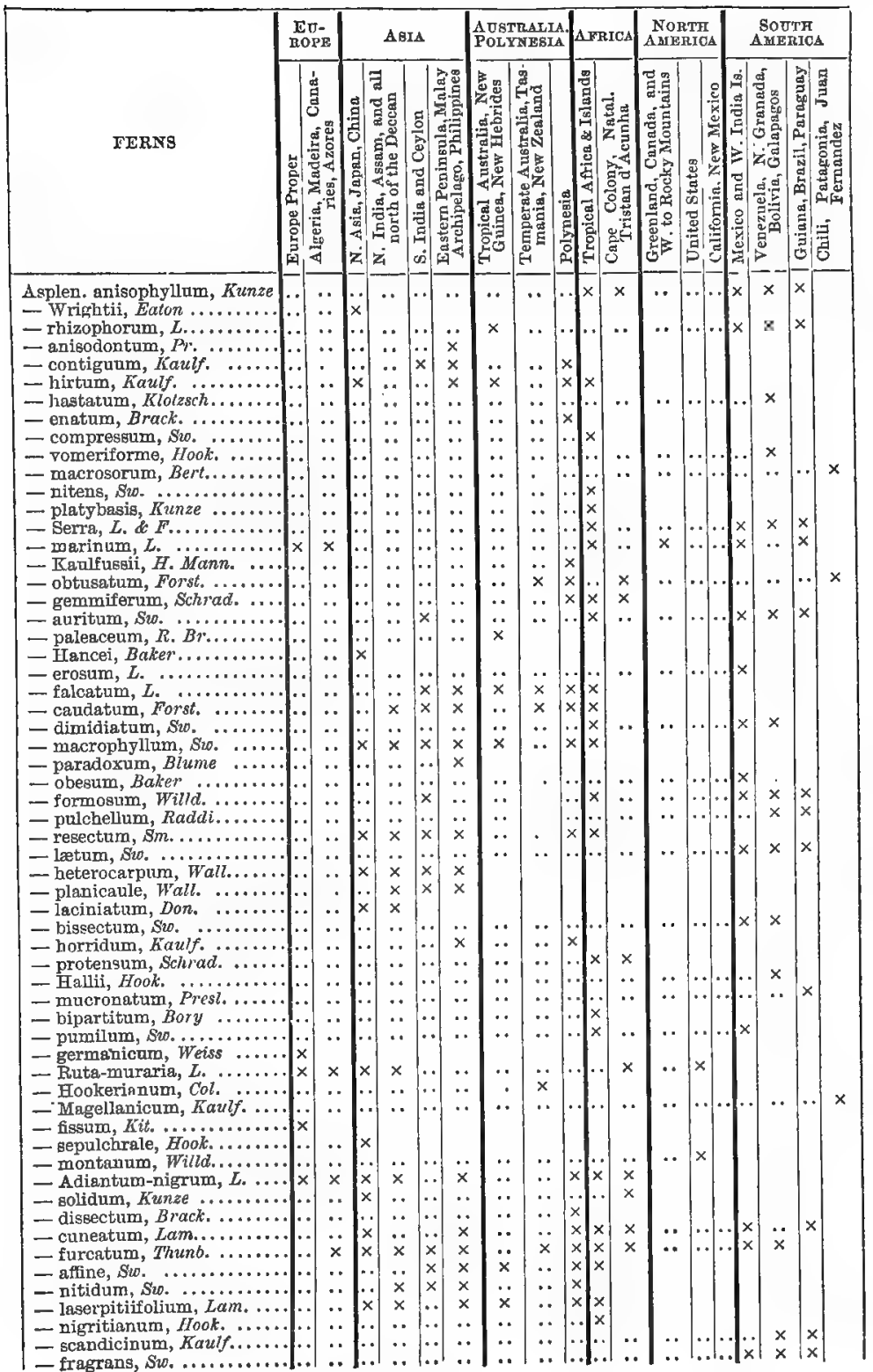




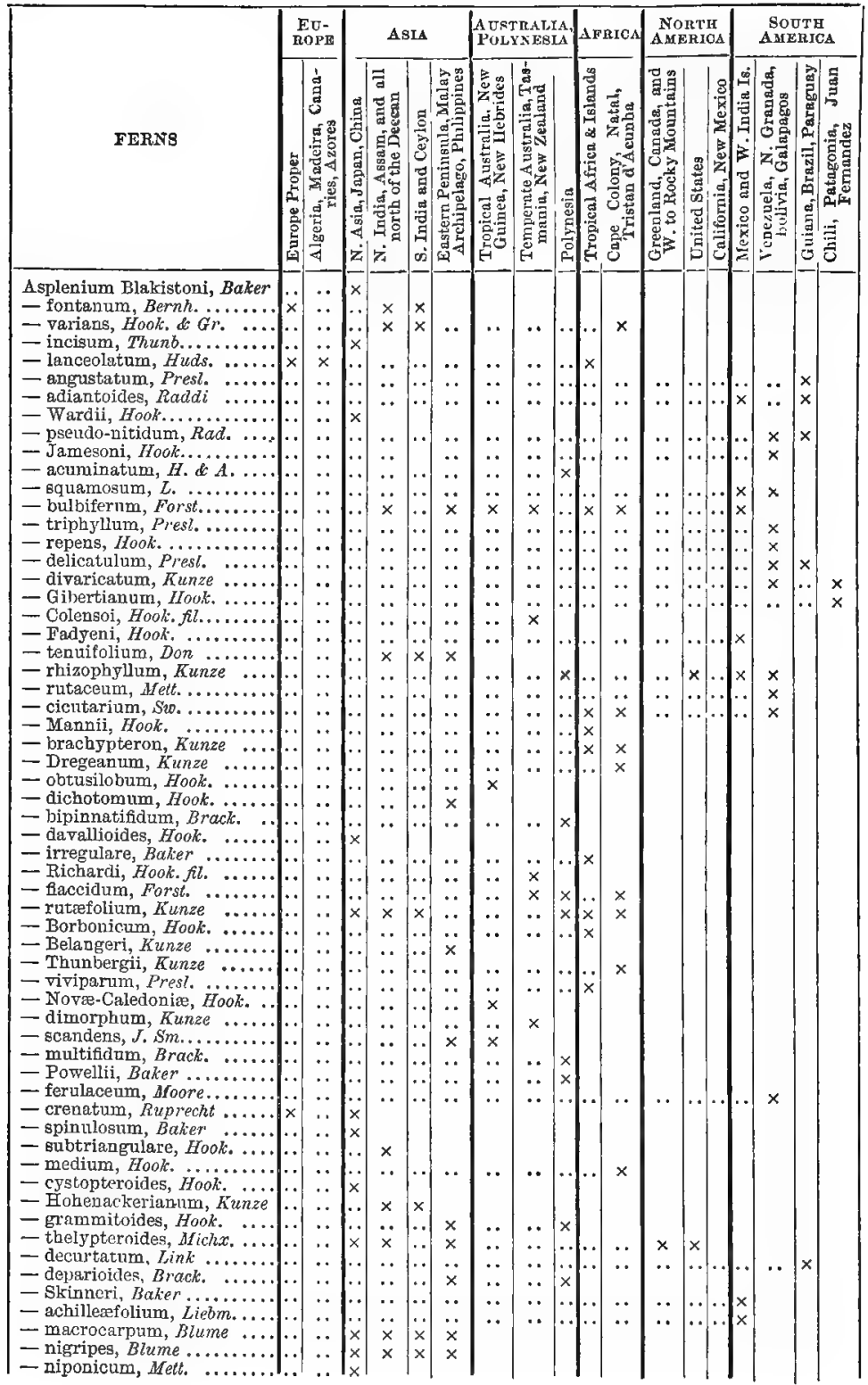




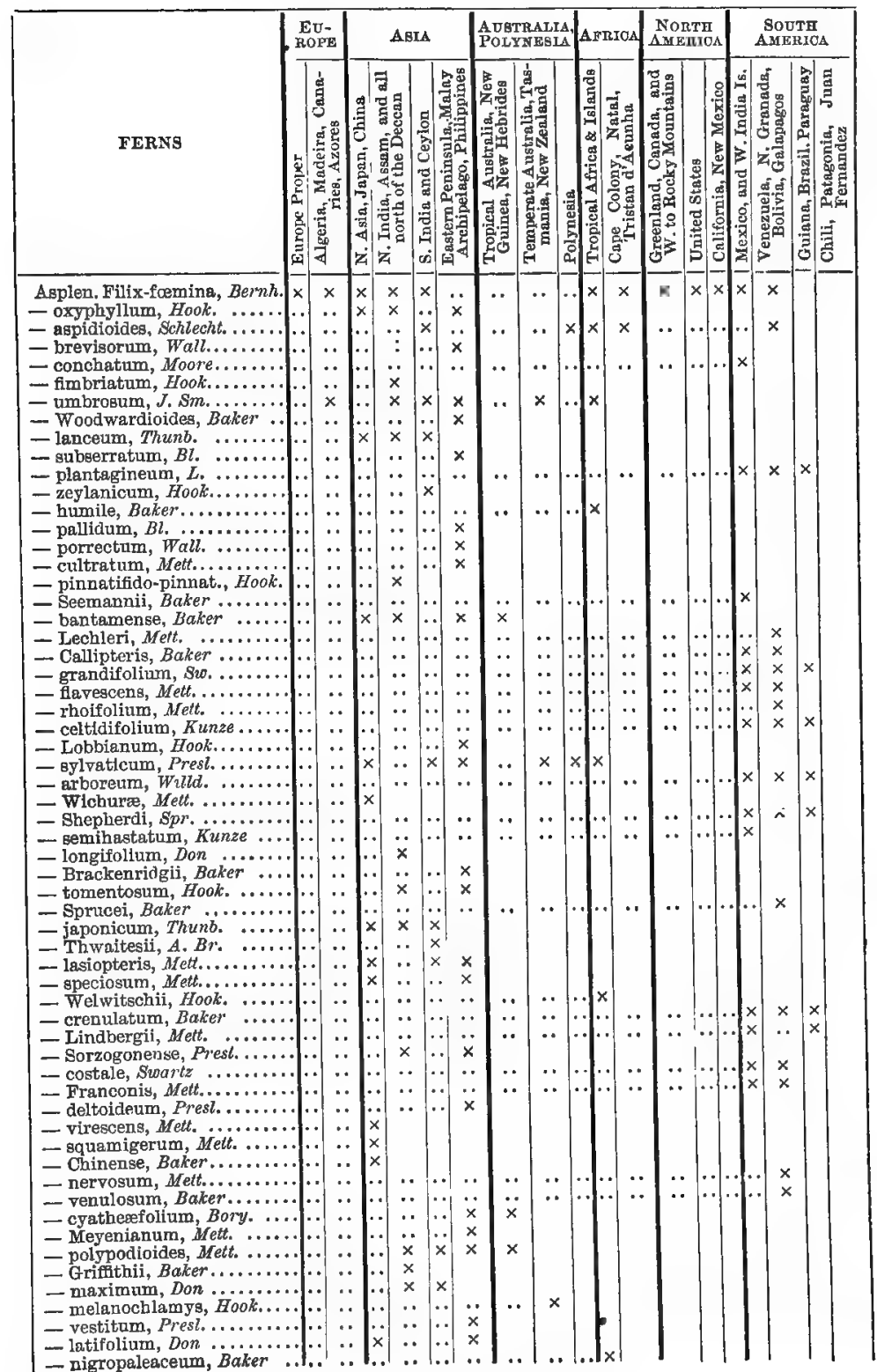




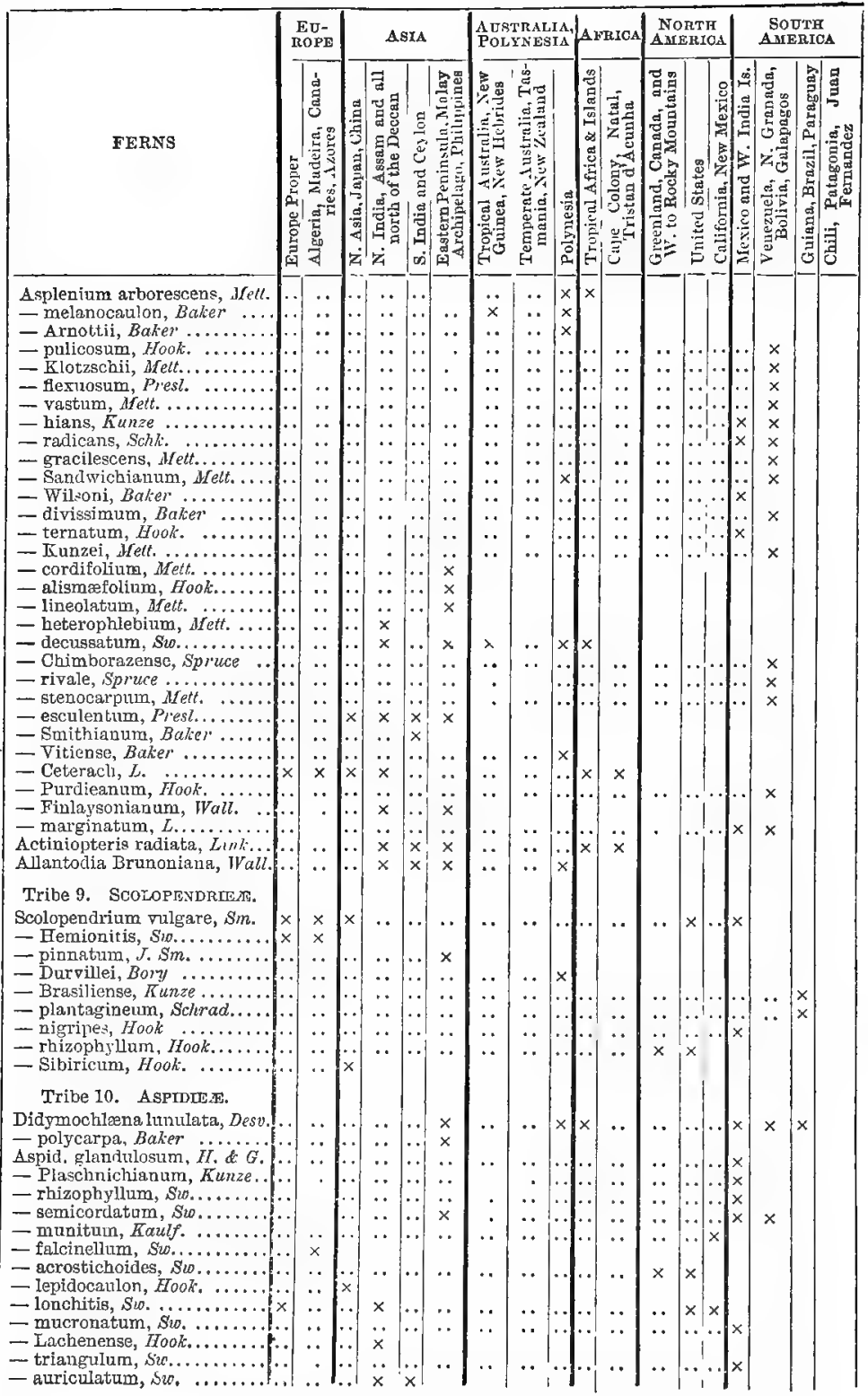




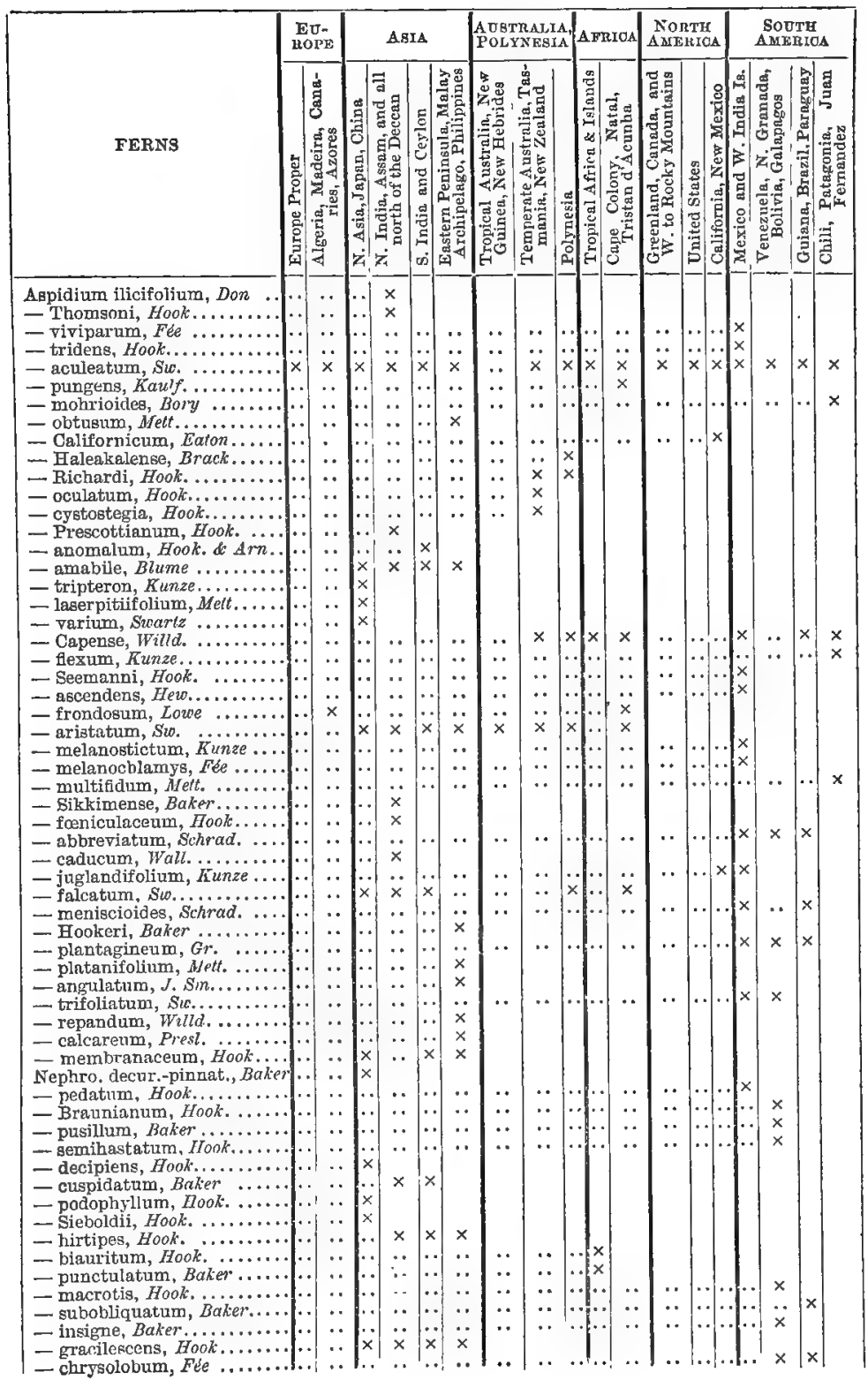




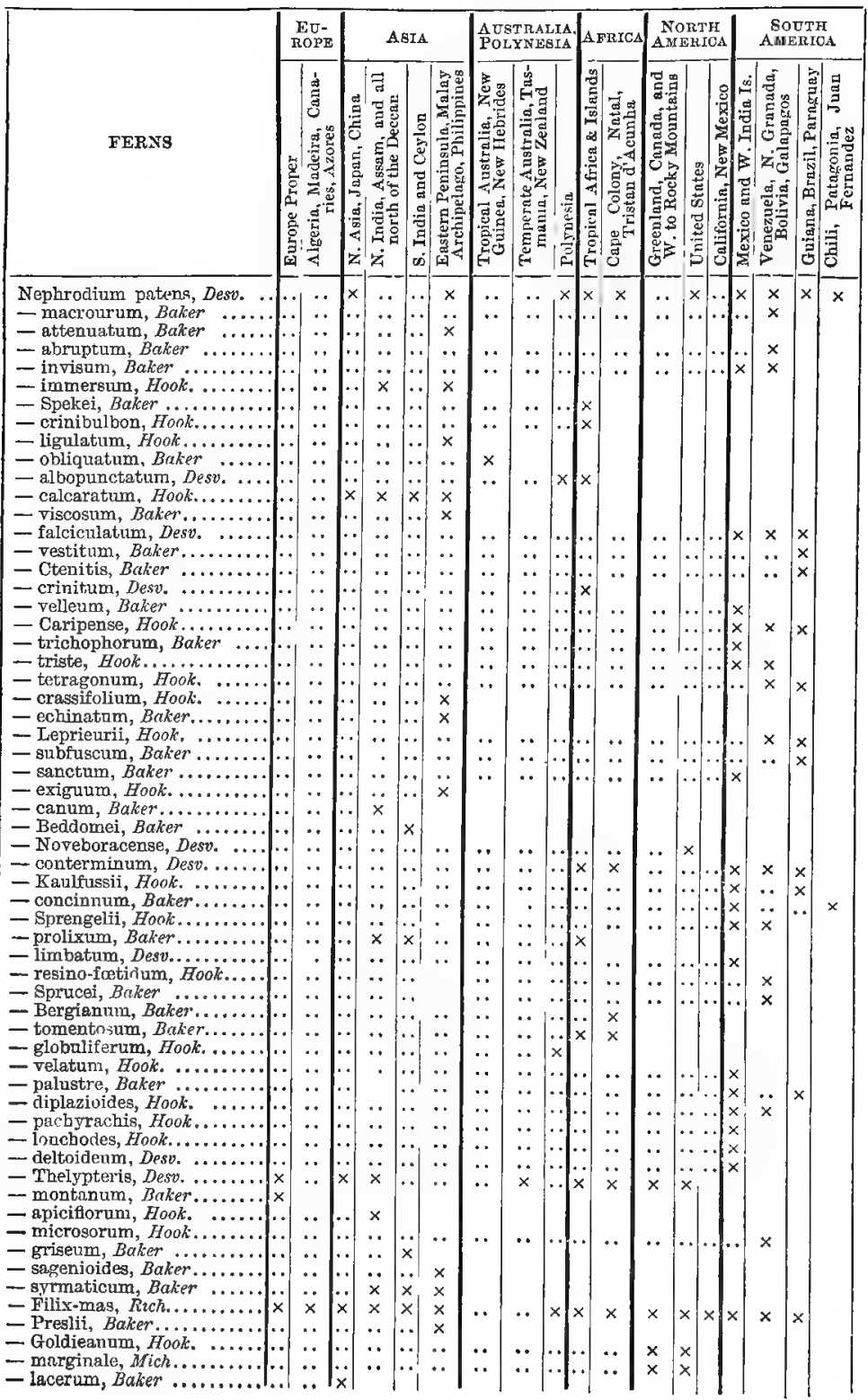




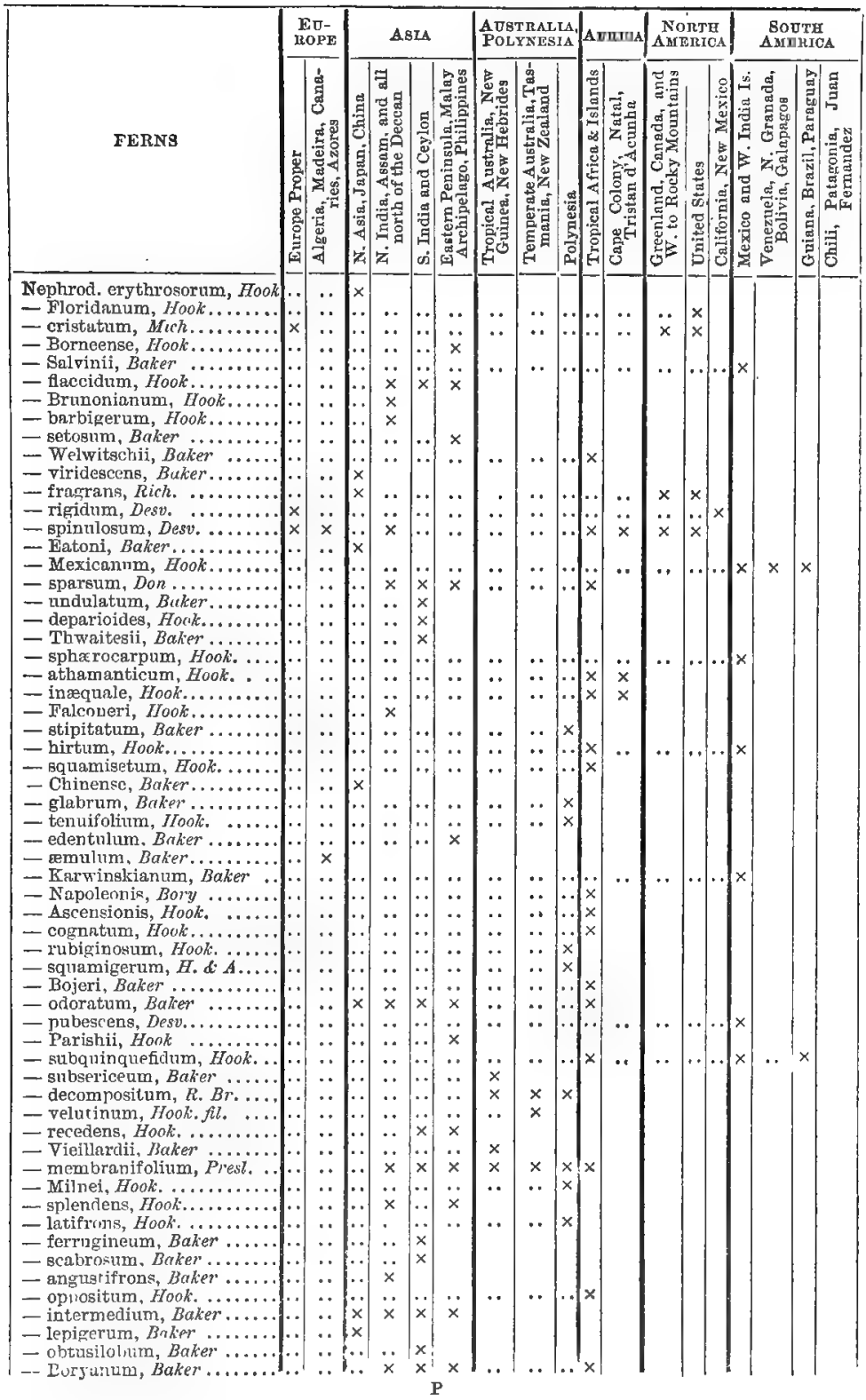




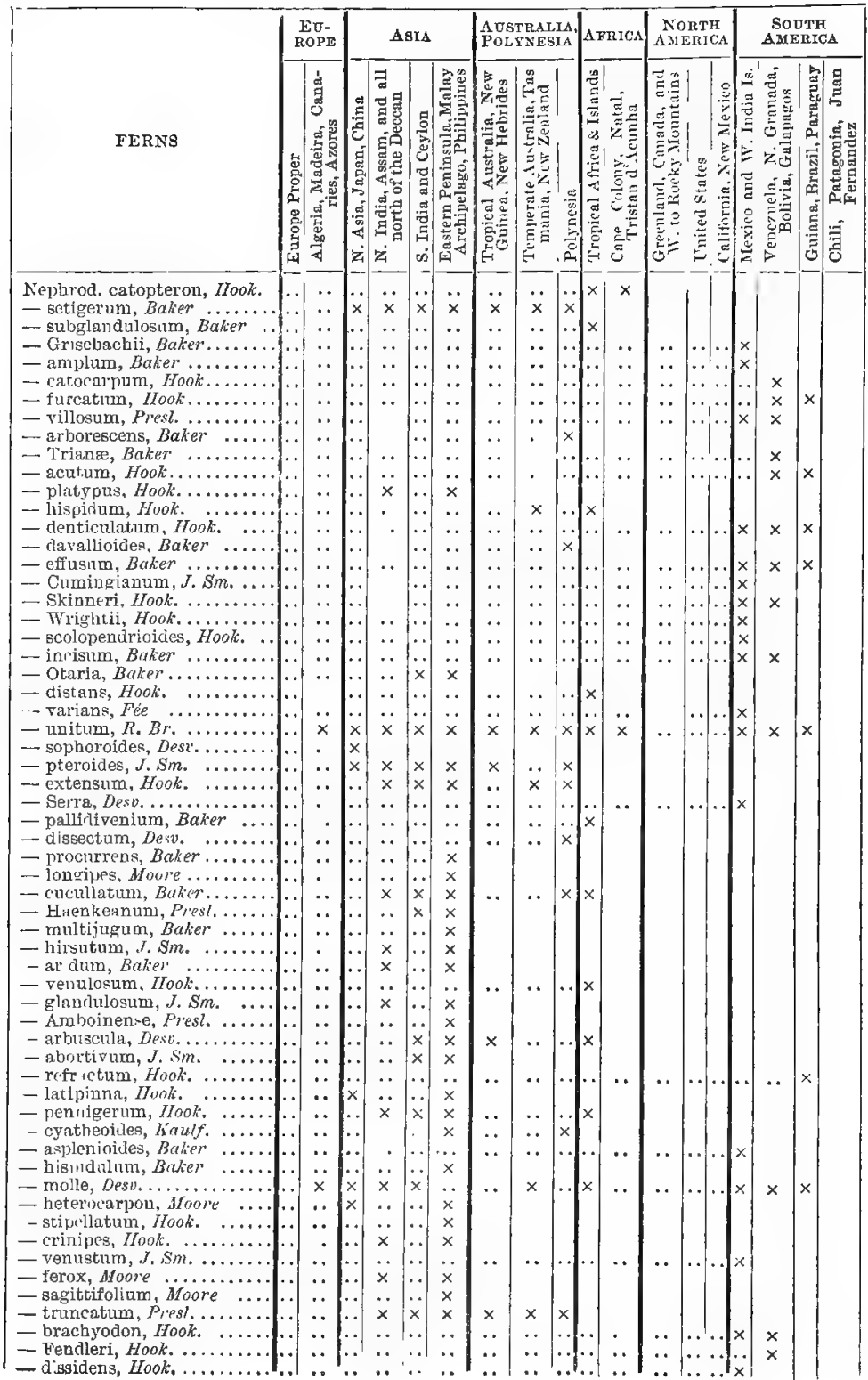




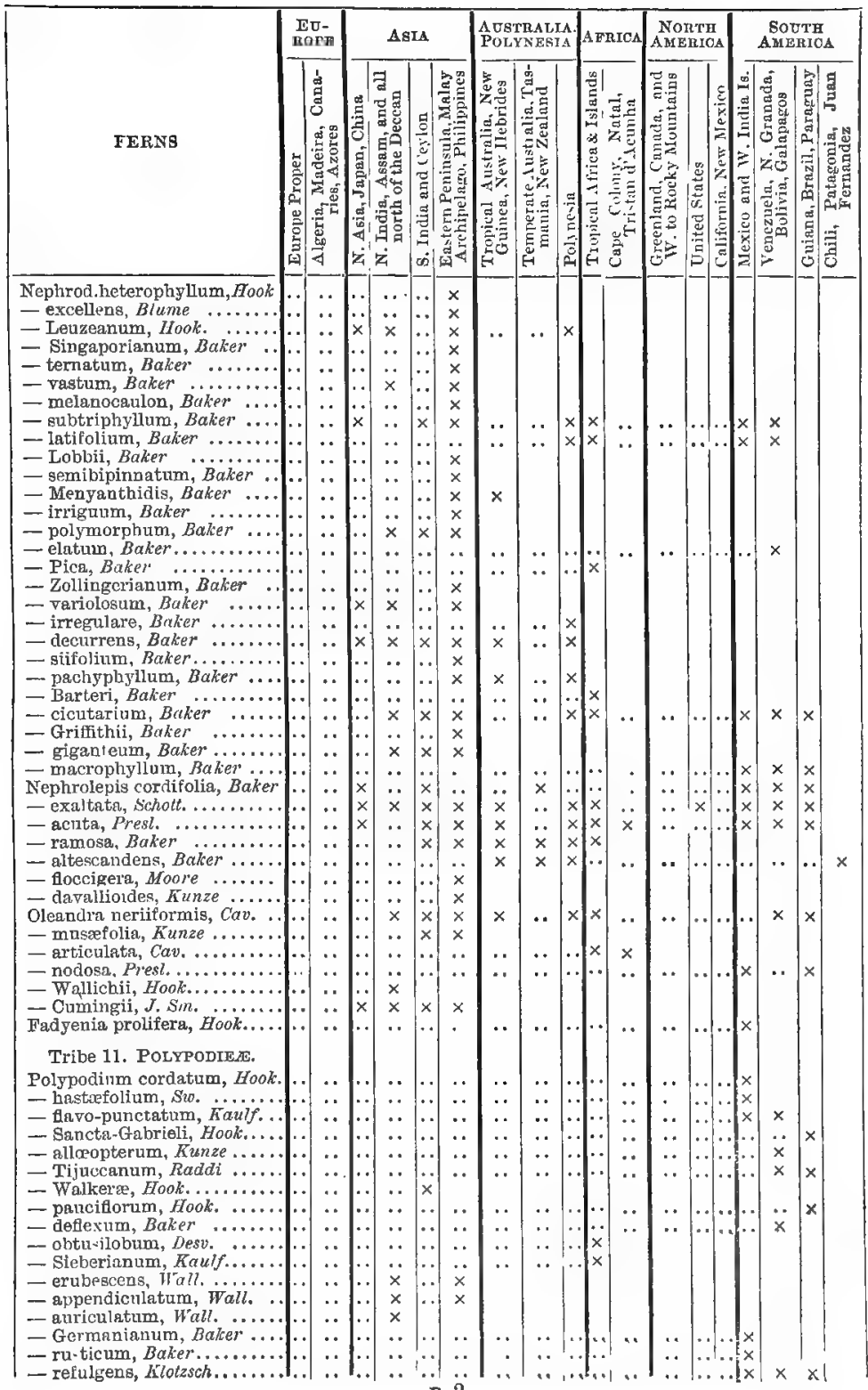




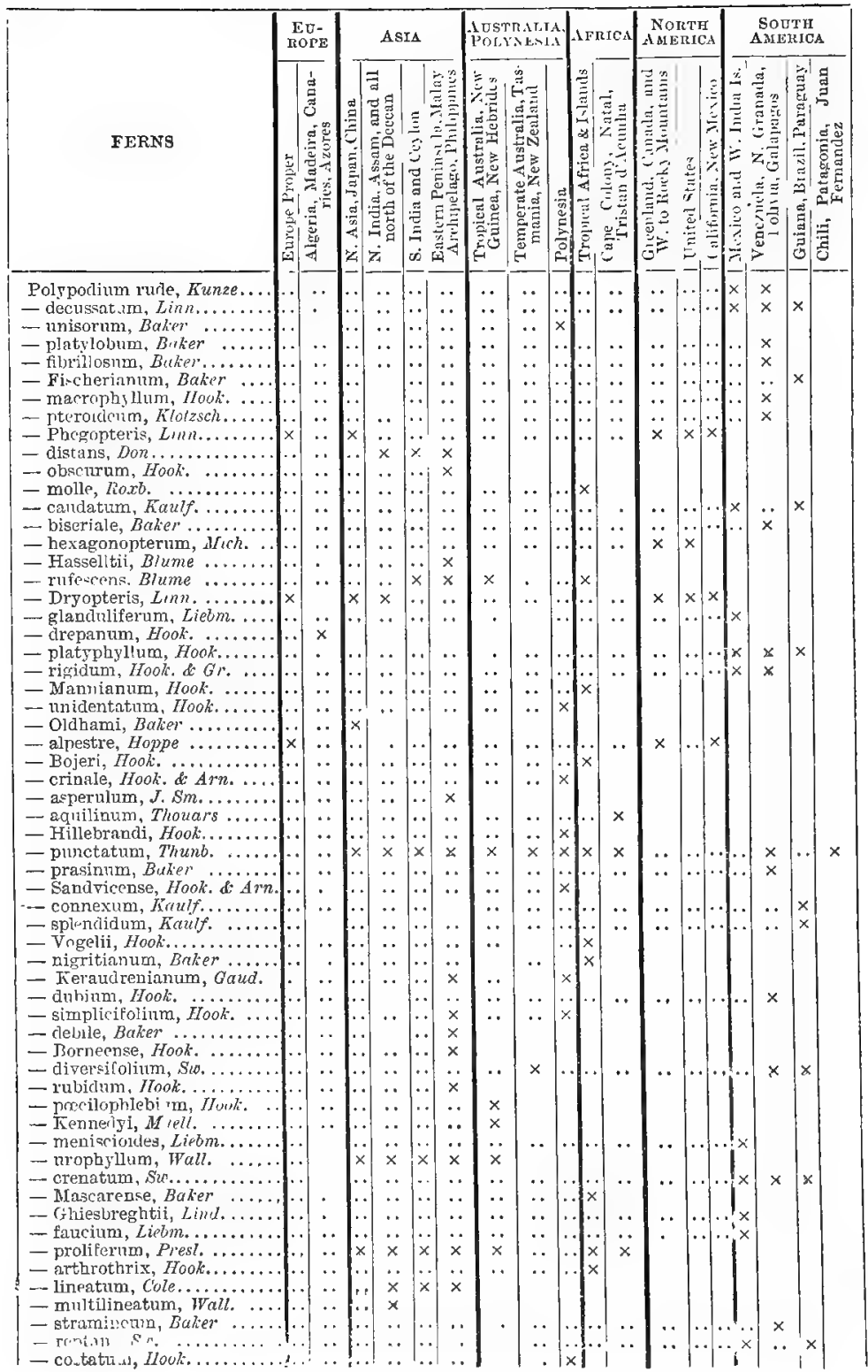




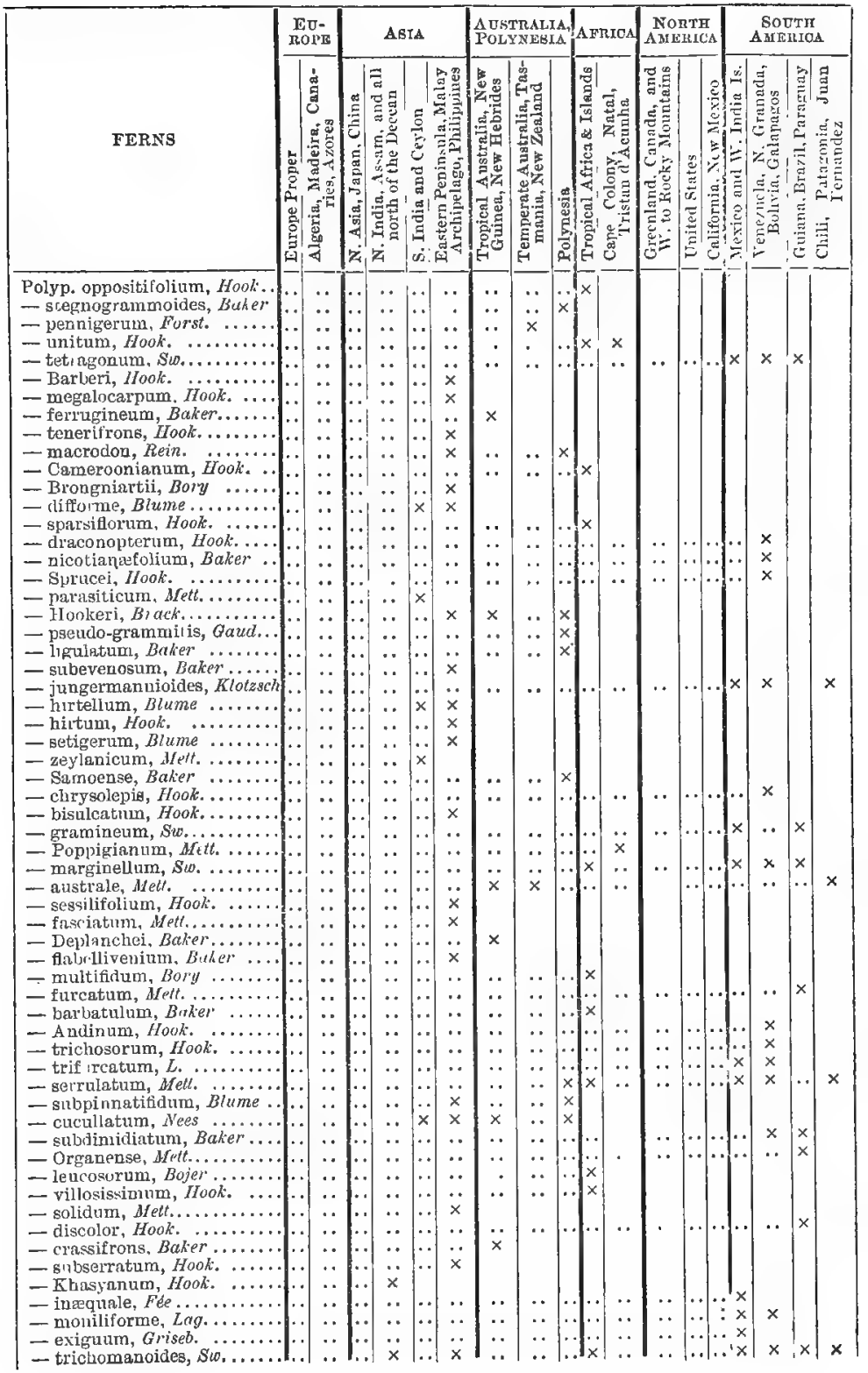




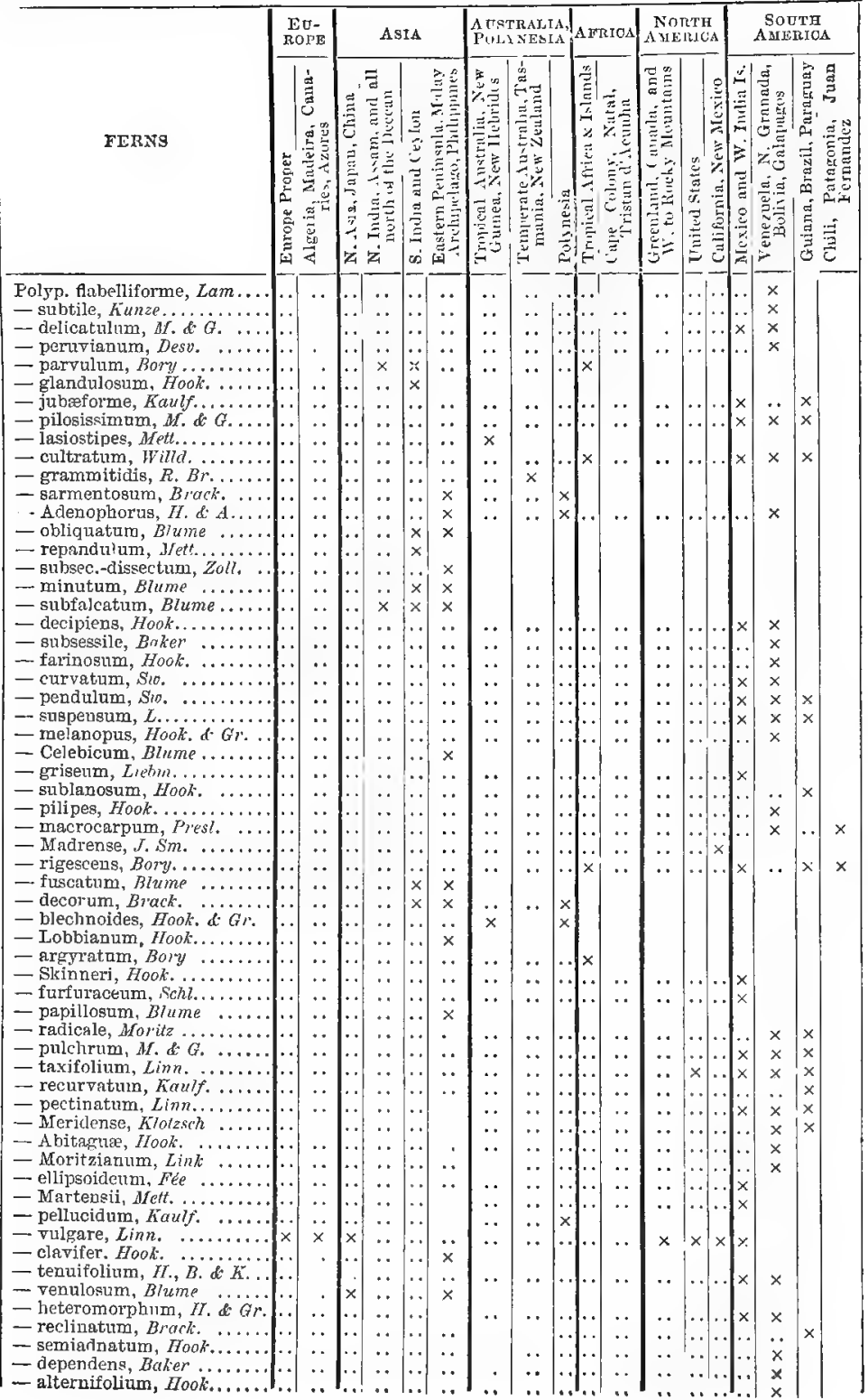




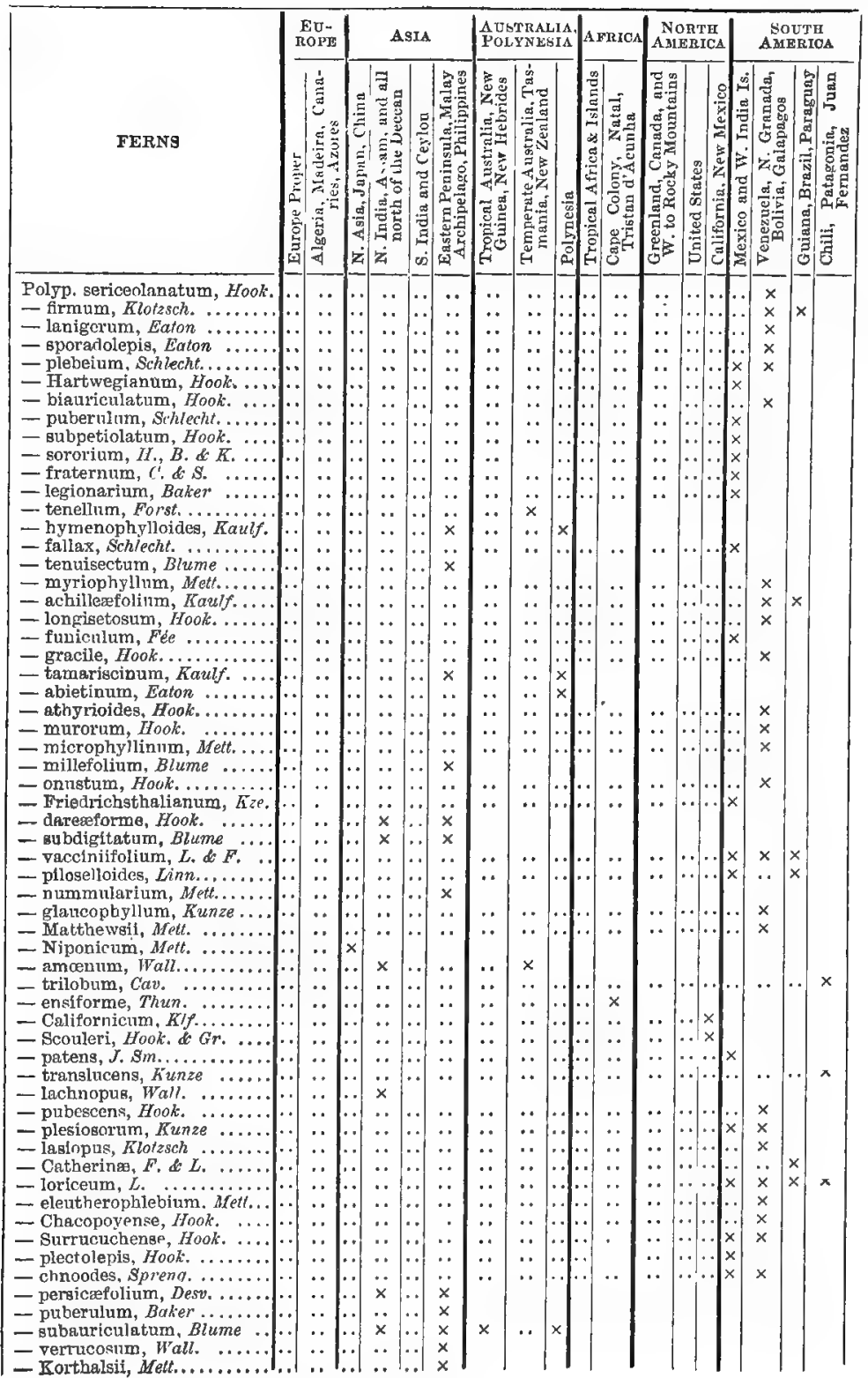




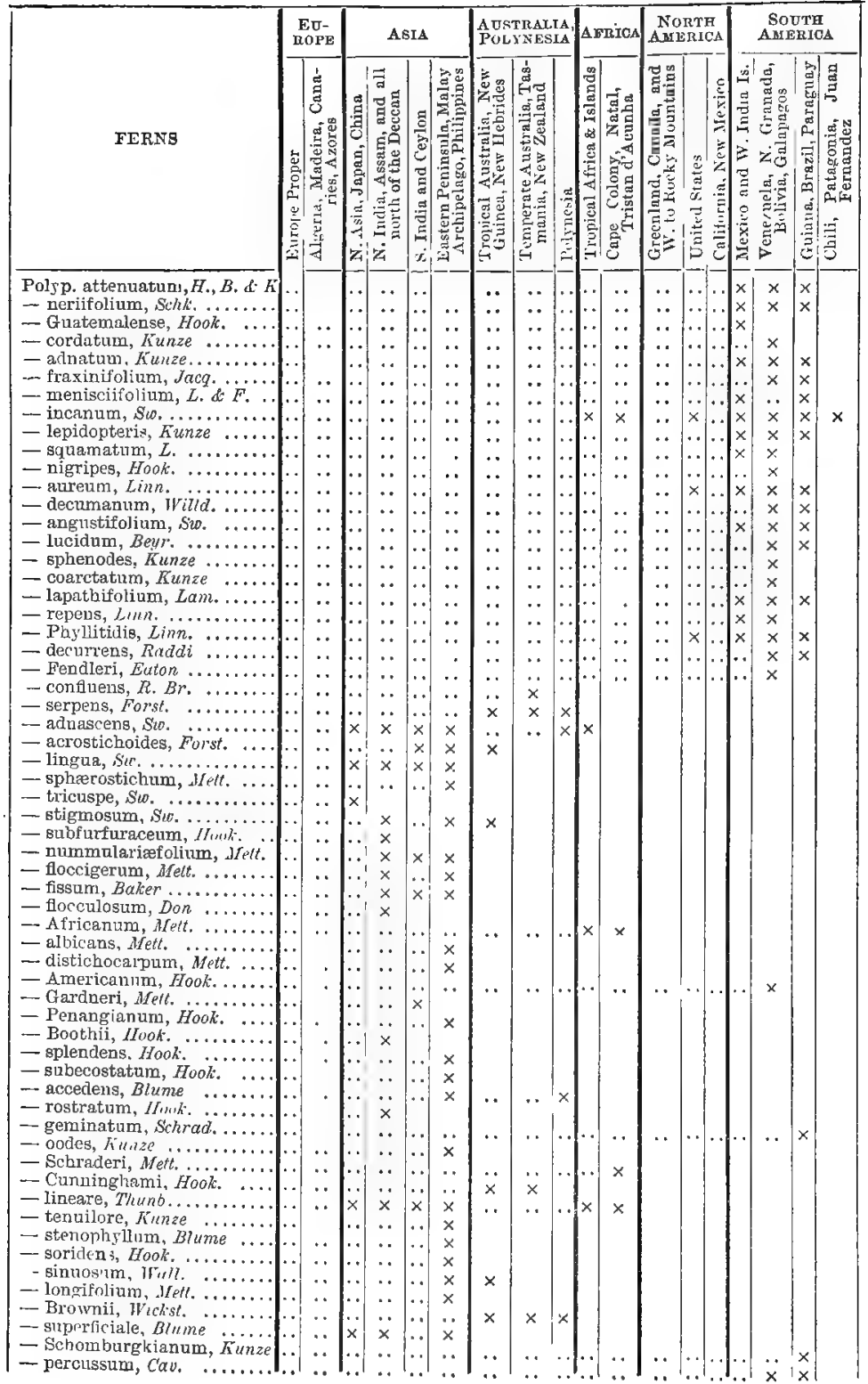




\begin{tabular}{|c|c|c|c|c|c|c|c|c|c|c|c|c|c|c|c|c|c|}
\hline \multirow[b]{2}{*}{ FERNS } & \multicolumn{2}{|c|}{$\underset{\text { ROPD }}{\text { ED- }}$} & \multicolumn{4}{|c|}{$A B I A$} & \multicolumn{3}{|c|}{$\begin{array}{l}\text { AUgTRaLLa } \\
\text { POLYNESIA }\end{array}$} & \multicolumn{2}{|c|}{ AEUICA } & \multicolumn{2}{|c|}{$\begin{array}{c}\text { NowTH } \\
\text { AMERICA }\end{array}$} & & \multicolumn{3}{|c|}{$\begin{array}{l}\text { SoUTH } \\
\text { AMERICA }\end{array}$} \\
\hline & 焉 & 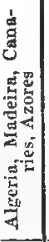 & & 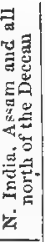 & $\mid$ & 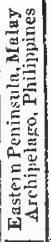 & 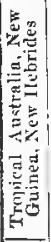 & 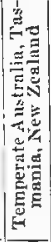 & \begin{tabular}{|c|}
$\mid$ \\
0 \\
0 \\
0 \\
0 \\
0 \\
0
\end{tabular} & 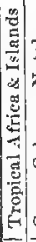 & 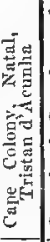 & 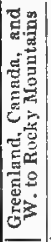 & \begin{tabular}{|c|c|} 
\\
\end{tabular} & 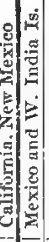 & 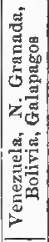 & 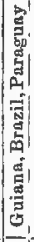 & 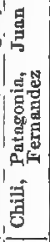 \\
\hline Polyp. lanceolatum, Linn... & $\cdots$ & $\ldots$ & .. & $\cdots$ & $x$ & . & . & $\cdots$ & $|x|$ & $x$ & $x$ & $\cdots$ & $\ldots$ & $\cdots \times$ & $x$ & $x$ & $\boldsymbol{\lambda}$ \\
\hline - linearifolium, Hook. ...... & $\therefore$ & $\cdots$ & $x$ &. & & 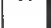 & . & 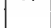 & & & 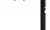 & 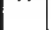 & & 7 & 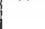 & & \\
\hline - glabrum, Mett. ......... & .. & $\cdots$ & $\cdots$ & $\because$ & $\because$ & $\cdots$ & $x$ & $x$ & & & & & & & & & \\
\hline — nngustatium, Swo, $\ldots \ldots \ldots$ & $\ldots$ & .. & ... & $x$ & $x$ & $x$ & $x$ & $x$ & $x$ & & & & & & & & \\
\hline $\begin{array}{l}\text { - Samarense, Mett, ....... } \\
\text { — Swartzii, Baker. . }\end{array}$ & .. & $\cdots$ & $\cdots$ & $\cdots$ & $\cdots$ & $x$ & & & & & & & & & & & \\
\hline 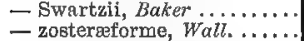 & .. & $\cdots$ & $\because$ & . & $\cdots$ & $\cdots$ & $\cdots$ & $\therefore$ & $\cdots$ & $\cdots$ & $\cdots$ & $\cdots$ & $\cdots$ & $\cdot x \mid$ & $\cdots$ & $x$ & \\
\hline $\begin{array}{l}\text { - zosteraforme, Wall. ....... } \\
\text { - stigmaticum, Presl. ...... }\end{array}$ & $\cdots$ & .. & $\therefore$ & $\cdots$ & $\cdots$ & $x$ & & & & & & & & & & & \\
\hline $\begin{array}{l}\text { - atigmaticum, Presl. . . . . } \\
\text { - lycopodioides, } L . \ldots \ldots\end{array}$ & $\cdots$ & .. & $\cdots$ & $*$ & $\cdots$ & 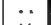 & $\cdots$ & $\because$ & $\cdots$ & 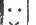 & $\ddot{q}$ & .. & $\cdots$ & $\cdots$ & $x$ & & \\
\hline 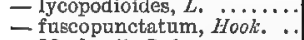 & $\cdots$ & $\cdots$ & 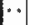 & $\cdots$ & $\cdots$ & $x$ & $\cdots$ & $\because$ & $x$ & $x$ & $x$ & $\cdots$ & $\cdots \cdot \cdots$ & $\cdots \times$ & $x$ & $x$ & \\
\hline - Iuscopunctálum, Hook. . & $\because$ & $\because 4$ & $\because$ & $\because$ & $\because$ & $\cdots$ & $\because$ & $\because$ & $\because$ & $\because$ & $\ddot{x}$ & $\because$ & $\because \cdot$ & $\cdots$ & $x$ & & \\
\hline - Iindbergii, Melt. $\quad \ldots \ldots \ldots$ & $\because$ & $\ddot{m}$ & $\because \cdot$ & $\ddot{*}$ & $\ddot{\cdots}$ & $\because$ & $\ddot{*}$ & $\because$ & $\cdots$ & $\therefore$ & . & $\cdots$ & $\ldots$. & $\cdots$ & $\cdots$ & $x$ & \\
\hline - persicaxiafolium, Schrad. & $\therefore$ & . & .. & $\cdots$ & .. & $\therefore$ & $\cdots$ & . & $\cdots$ & $\cdots$ & $\ddot{*}$ & $\cdots$ & $\ldots$ & $\cdot x$ & $\mathbf{x}$ & $\mathbf{x}$ & \\
\hline - zormale, Don ............ & $\cdots$ & . & $x$ & $x$ & $\cdots$ & $x$ & $\cdots$ & $\cdots$ & $\cdots$ & $\cdots$ & $x$ & & & & & & \\
\hline $\begin{array}{l}\text { - myriocarpum, Meth. ...... } \\
\text { - linguæforme, Mett. } . . . . .\end{array}$ & $\cdots$ & $\cdots$ & $\cdots$ & $\cdots$ & $\cdots$ & $x$ & & & & & & & & & & & \\
\hline $\begin{array}{l}\text { - linguaforme, Mett. } \ldots \ldots \ldots \\
\text { - lancevla, Melt. . . . . . . }\end{array}$ & $\cdots$ & $\cdots$ & $\cdots$ & $\cdots$ & $\cdots$ & $\times$ & $x$ & & & & & & & & & & \\
\hline & $\cdots$ & $\cdots$ & $\cdots$ & $\because$ & .. & $\because$ & $x$ & & & & & & & & & & \\
\hline - rhyncophyllum, Hook..... & $\because$ & $\because$ & $\cdots$ & $\begin{array}{l}x \\
x\end{array}$ & 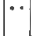 & $x$ & & & & & & & & & & & \\
\hline - Melleri, Baker ......... & $\ldots$ & $\therefore$ & $\therefore$ & $\cdots$ & $\cdots$ & - & $\cdots$ & $\cdots$ & $\cdots$ & $x$ & & & & & & & \\
\hline — triquetrum, Blume ...... & $\cdots$ & . & $\cdots$ & $\cdots$ & . & $x$ & & & & & & & & & & & \\
\hline - rupestre, Blume..... . . . & $\cdots$ & $\cdots$ & $\cdots$ & $\ddot{x}$ & $\cdots$ & $x$ & & & & & & & & & & & \\
\hline - ovatam, Wall. .......... & $\cdot$ & *. & $\cdots$ & $x$ & & & & & & & & & & & & & \\
\hline $\begin{array}{l}\text { - platyphyllum, Sw. } \ldots \ldots \ldots \\
\text { - crassifolium, } L . \ldots \ldots \ldots \ldots \ldots\end{array}$ & $\cdots$ & $\cdots$ & $\cdots$ & $\because$ & $\cdots$ & $x$ & & & & & & & & & & & \\
\hline $\begin{array}{l}\text { - crassifolium, } L, \ldots \ldots \ldots \ldots \\
\text { - membranaceum, } \text { Don } \ldots \ldots\end{array}$ & $\cdots$ & $\cdots$ & $\cdots$ & $\ddot{x}$ & $\ddot{x} \mid$ & $\cdots$ & - & $\cdots$ & $\cdots$ & $\because$ & $\cdots$ & $\cdots$ & $\cdots$ & $\times$ & $x$ & $x$ & \\
\hline - heterocarpum, Blume .... & $\because \cdots$ & $\because$ & $\cdots$ & $\begin{array}{l}x \\
x\end{array}$ & $x$ & $x$ & & & & & & & & & & & \\
\hline - irioides, Lam. .......... & $\ldots$ & . & $x \mid$ & $x$ & $x$ & $x$ & $x$ & $\because$ & $x$ & $x$ & $x$ & & & & & & \\
\hline - musefolium, Blume $\ldots$... & $\cdots$ & $\cdots$ & $\cdots$ & 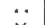 & $\because$ & $x$ & & & & & & & & & & & \\
\hline $\begin{array}{l}\text { — hemionitideum, Wall. . . . } \\
\text { — ensatum, Thunb. ........ }\end{array}$ & .. & - & $\ddot{x}$ & $x$ & $x$ & & & & & & & & & & & & \\
\hline $\begin{array}{l}\text { - ensatum, Thunb, } \ldots \ldots \ldots . \\
\text { - crispatum, Book. } \ldots \ldots \ldots\end{array}$ & $\cdots$ & $\cdots$ & $\mathrm{x}$ & & & & & & & & & & & & & & \\
\hline $\begin{array}{l}\text { - crispatum, Hook. } \ldots \ldots \ldots \\
\text { - Labrusca, Hook. }\end{array}$ & $\cdots$ & & $\cdots$ & $\cdots$ & $\cdots$ & $\because$ & $\cdots$ & $\because$ & $\cdots$ & $\cdots$ & $\cdots$ & $\cdots$ & • & $x$ & & & \\
\hline $\begin{array}{l}\text { - Labrusca, Hook. } \quad \ldots \ldots \ldots \\
\text { - bifrons, Hook. ........ }\end{array}$ & $\cdots$ & ? & $\cdots$ & - & $\cdots$ & $x$ & & & & & & & & & & & \\
\hline 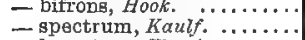 & a & $\cdots$ & $\cdots$ & $\cdots$ & $\cdots$ & $\ddot{x}$ & $\because$ & $\because$ & $\ddot{x}$ & $\cdots$ & $\cdots$ & $\because$ & $\cdots$ & $\because$ & $x$ & & \\
\hline - Spectrum, Kaubf. ....... & $\because$ & $\because$ & $\ddot{x}$ & $\cdots$ & $\cdots$ & $x$ & $\cdots$ & $\because$ & $\lambda$ & & & & & & & & \\
\hline - pteropus, Biume ....... & ... & ․ & $x$ & $x$ & $x$ & $x$ & & & & $\vdots$ & & & & & & & \\
\hline - Dipteris, Blume ..... & $\cdots$ & $\cdots$ & $\cdots$ & 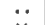 & $\cdots$ & $x$ & $x$ & $\cdots$ & $x$ & & & & & & & & \\
\hline $\begin{array}{l}\text { - Wallichii, R. Br. .... } \\
\text { - Lobbiunum, Hook. .. }\end{array}$ & $\cdots$ & $\because$ & $\cdots$ & $x$ & & & & & & & & & & & & & \\
\hline $\begin{array}{l}\text { - Lobbiunum, Hook. ..... } \\
\text { - leucosporum, Klotzsch } \ldots .\end{array}$ & $\cdots$ & $\because$ & $\because$ & $\cdots$ & $\cdots$ & $x$ & & & & & & & & & $x$ & & \\
\hline & $\because$ & $\ddot{*}$ & $\because$ & $\ddot{x}$ & $\ddot{x}$ & $\cdots$ & $\cdots$ & 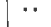 & $\cdots$ & $\cdots$ & $\cdots$ & $\cdots$ & - & & n & & \\
\hline $\begin{array}{l}\text { - trificum, Don ............ } \\
\text { — malacodot, Hook......... }\end{array}$ & $\because$ & $\because$ & $\because$ & $\begin{array}{l}x \\
x\end{array}$ & $x$ & & & & & & & & & & & & \\
\hline - malacodon, Hook......... & . . & $\cdots$ & ... & $\cdots$ & $\cdots$ & $x$ & & & & & & & & & & & \\
\hline - angustum. Mett. $\ldots \ldots$ & $\cdots$ & $\because$ & .. & . & $\cdots$ & 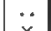 & $\cdots$ & . & $\cdots$ & $\cdots$ & • & $\because$ & $\cdots$ & $\cdots$ & “. & $x$ & \\
\hline - insigne, Blume ...... &.. & $\because$ & $\cdots$ & . & $\cdots$ & $x$ & & & & & & & & & & & \\
\hline - pustulatum, Forst. ...... & $\cdots$ & $\cdots$ & $\because$ & $\cdots$ & $\cdots$ & $\because$ & $x$ & $x$ & & & & & & & & & \\
\hline - Billardieri, $R_{.} B r . \quad \ldots \ldots$ & $\cdots$ & .. & $\because$ & $\cdots$ & 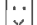 & $\ddot{v}$ & $\ddot{x}$ & $x$ & & & $x$ & & & & & & \\
\hline 一 phymatodes, $L . \ldots \ldots \ldots \ldots$ & $\cdots$ & $\cdots$ & $x$ & $\because$ & $x$ & $x$ & $x$ & & $x$ & $x$ & $x$ & & & & & & \\
\hline - nigrescens, Blume ........ & $\cdots$ & $\cdots$ & $\cdots$ & $x$ & $x$ & $x$ & $\cdots$ & - & $x$ & & & & & & & & \\
\hline $\begin{array}{l}\text { - affine, Blume ............. } \\
\text { - Powellii, Baker ........... }\end{array}$ & $\cdots$ & $\because$ & $\because$ & $\cdots$ & $\cdots$ & $x$ & & & $x$ & & & & & & & & \\
\hline $\begin{array}{l}\text { - Powellii, Baker ........... } \\
\text { - dilatatım, Wall. ....... }\end{array}$ & $\because$ & 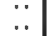 & $\because$ & $\ddot{x}$ & $\because$ & $\ddot{x}$ & $\because$ & $\because$ & $x$ & & & & & & & & \\
\hline - dilatatum, Wall. ....... &.. & $\therefore$ & $\because$ & $\begin{array}{l}x \\
\cdots\end{array}$ & $\cdots$ & $\begin{array}{l}x \\
x\end{array}$ & .. & & & & & & & & & & \\
\hline $\begin{array}{l}\text { - laciniatum, Blume } \ldots \ldots \\
\text { - alatum, Hook. .......... }\end{array}$ &.. & . & $\because$ & $\because$ & $\ldots$ & . & $\cdots$ & & $x$ & & & & & & & & \\
\hline - glancum, Kunze........... & $\cdots$ & $\cdots$ & . & .. & $\cdots$ & $x$ & & & & & & & & & & & \\
\hline - lomarioides, Kunze ..... & $\cdots$ & $\cdots$ & $\cdots$ & $\because$ & $\cdots$ & $x$ & & & & & & & & & & & \\
\hline - ebenipes, Jlook. .......... & $\because$ & $\cdots$ & $\ddot{x}$ & $x$ & & & & & & & & & & & & & \\
\hline - longis simum. Blume .... & $\because$ & $\ddot{m}$ & $\mid x$ & $x$ & $x$ & $\begin{array}{l}x \\
x\end{array}$ & & & & & & & & & & & \\
\hline
\end{tabular}




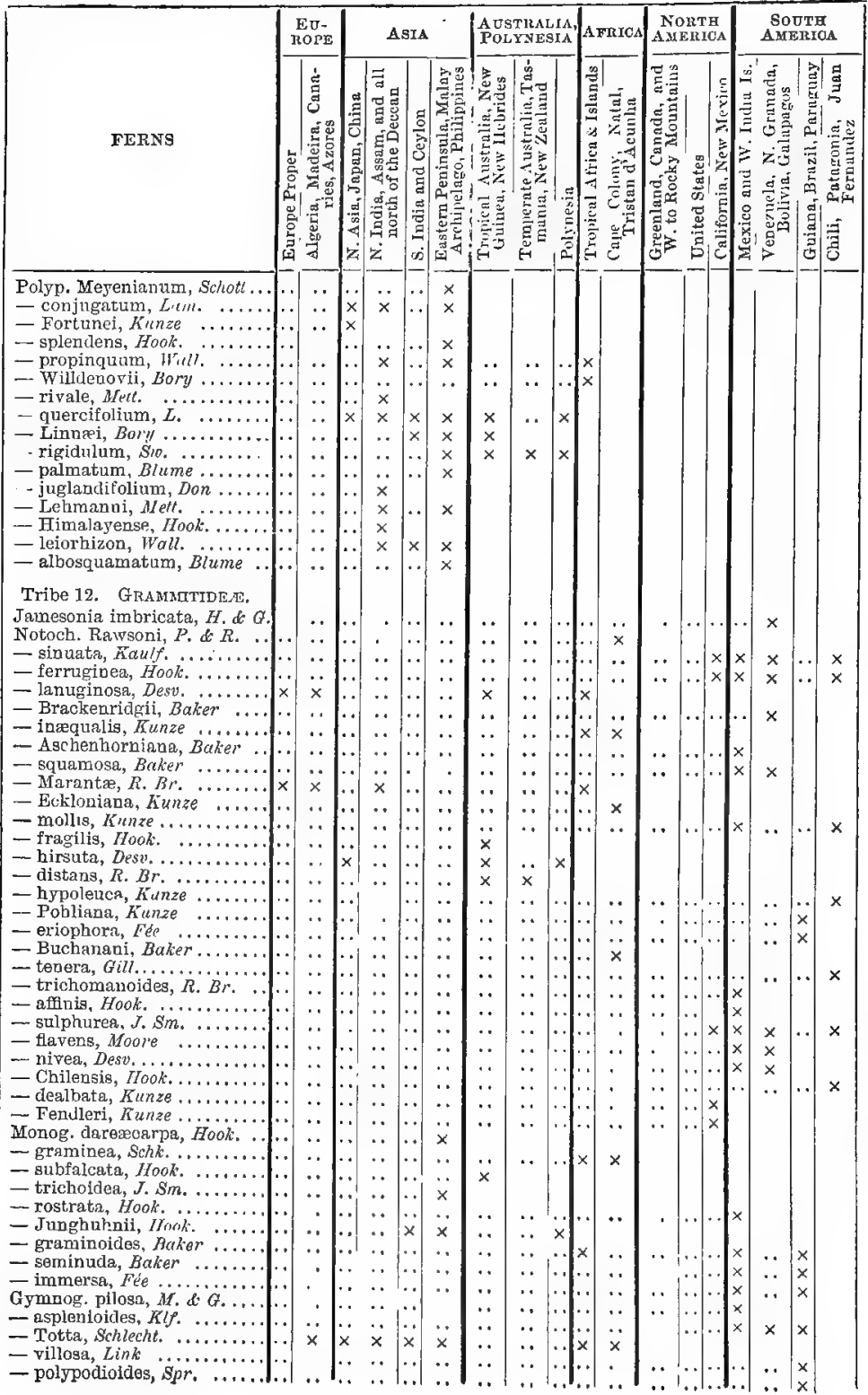




\begin{tabular}{|c|c|c|c|c|c|c|c|c|c|c|c|c|c|c|c|c|c|}
\hline \multirow[b]{2}{*}{ FERNS } & \multicolumn{2}{|c|}{$\underset{\text { 12OPB }}{\mathrm{EU}}$} & \multicolumn{4}{|c|}{ AsIA } & \multicolumn{3}{|c|}{$\mid \begin{array}{l}\text { AUGTRATIA, } \\
\text { POLYNESIA }\end{array}$} & \multicolumn{2}{|c|}{ AFRICA } & \multicolumn{2}{|c|}{$\begin{array}{c}\text { NORTH } \\
\text { AMEMICA }\end{array}$} & \multicolumn{4}{|c|}{$\begin{array}{c}\text { SOUTH } \\
\text { AMERIOA }\end{array}$} \\
\hline & 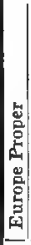 & 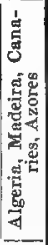 & 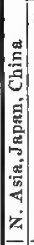 & 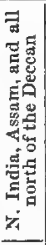 & 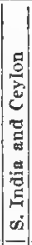 & 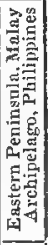 & 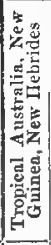 & 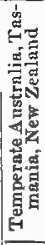 & 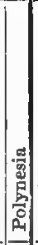 & 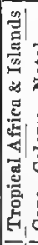 & 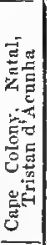 & 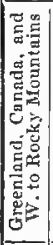 & 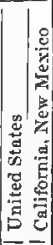 & 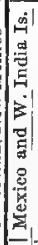 & 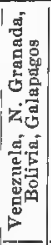 & 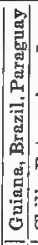 & 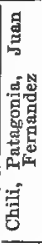 \\
\hline Gymnog. diplazioides, Desv. & $\ldots$ & .. &.. & .. & $\cdots$ & & & $\cdots$ & & & & & & $x$ & $x$ & $x$ & \\
\hline - grandis, Baker & $\ldots$ & $\cdots$ & 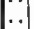 & 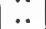 & $\because$ & . & $\because$ & 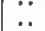 & $\because$ & $\because$ & $\ddot{0}$ & $\ddot{*}$ & & 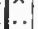 & $\hat{x}$ & $\alpha$ & \\
\hline - gracilis, Heward" & $\because$ & $\because$ & $\therefore$ & $\ddot{0}$ & $\because$ & $\ddot{*}$ & 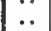 & $\because$ & $\cdots$ & & $\cdots$ & 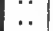 & & $\ddot{x}$ & $\hat{x}$ & & \\
\hline - Aurita, Hook. . . . & $\therefore$ & .. & $\therefore$ & $x$ & & $\cdots$ & & & & & & & & & & & \\
\hline - decurrenti-alata, Hook. . & $\cdots$ & .. & $x$ & & & & & & & & & & & & & & \\
\hline - opaca, Spreng. ......... & $\cdots$ & $\cdots$ & $\therefore$ & $x$ & .. & $x$ & & & & & & & & & & & \\
\hline - ambigua, Hook. ......... & .. & $\cdots$ & ... & $\cdots$ & .. & $x$ & & & & & & & & & & & \\
\hline - aspidioides, Hook. . . . . . . & $\cdots$ & $\cdots$ & . & $x$ & $x$ & $x$ & & & & & & & & & & & \\
\hline & $\because$ & $\because$ & . & $\cdots$ & $\cdots$ & $\cdots$ & $\ddot{x}$ & $\cdots$ & $\cdots$ & $\cdots$ & $\because$ & $\cdots$ & & . & $x$ & $x$ & \\
\hline $\begin{array}{l}\text { - marginata, Metl. } \ldots \ldots \ldots \\
\text { - pumila, Spreng. ......... }\end{array}$ & $\because$ & $\because$ & $\because$ & $\because$ & $\because$ & $\because$ & $x$ & & & & & & & & & & \\
\hline - Muelieri, Hook. . . . . . . . . & $\ldots$ & 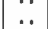 & $\because$ & $\because$ & $\because$ & $\because$ & $\ddot{x}$ & . & $\cdots$ & $\cdots$ & $\cdots$ & $\cdots$ & & $x$ & $\cdots$ & $\mathrm{x}$ & \\
\hline - vestita, Hook. ........ & $\ldots$ & .. & $x$ & $x$ & & & & & & & & & & & & & \\
\hline - rufa, Desv. .... & .. & $\cdots$ & .. & *. & $\cdots$ & $\cdots$ & $\cdots$ & $\cdots$ & .. & $\ldots$ & $\cdots$ & . & .. & $x$ & $x$ & $x$ & \\
\hline - cordata, Schlecht. . ....... & $\cdots$ & $\cdots$ & $\therefore$ & . & $\ldots$ & $\cdots$ & .. & $\cdots$ & . & $x$ & $x$ & & & & & & \\
\hline - Pozoi, Kunze .......... & $x$ & .. & . & $\cdots$ & $\cdots$ & - & $\cdots$ & $x$ & .. & 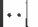 & $\cdots$ & $\cdots$ & $\cdots$ & $\cdots$ & *. & . . & $x$ \\
\hline - Andersoni, Beddome...... & $\cdots$ & . & $\cdots$ & $\times$ & & & & & & & & & & & & & \\
\hline - tomentosa, Desv. ....... & $\cdots$ & $\cdots$ & $\cdots$ & $\cdots$ & $\cdots$ & $\because$ & $\cdots$ & . & $\cdots$ & $\cdots$ & $\cdots$ & $\cdots$ & & - & $x$ & $x$ & \\
\hline - angustifrons, Baker ..... & $\cdots$ & $\cdots$ & $\cdot$. & $\cdots$ & $\cdots$ & $\cdots$ & .. & .. & .. & $\cdots$ & $\cdots$ & *. & & $\cdots$ & $x$ & & \\
\hline - hirsutula, Mett. $\ldots \ldots \ldots$. & $\cdots$ & $\cdots$ & $\cdots$ & $\cdots$ & $\cdots$ & $\cdots$ & $\because$ & $\cdots$ & $\cdots$ & $\cdots$ & $\cdots$ & $\cdots$ & & 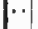 & $\times$ & & \\
\hline - Karstenüi, Melt. . . . . . . & $\because$ & $\because$ & $\because$ & $\because$ & $\cdots$ & $\because$ & $\cdots$ & $\because$ & $\because$ & $\because$ & $\cdots$ & $\cdots$ & & & $\underset{x}{x}$ & & \\
\hline 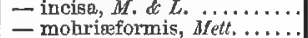 & $\because$ & $\ddot{m}$ & $\left|\begin{array}{l}\cdots \\
. .\end{array}\right|$ & $\because$ & $\because$ & $\ddot{.}$ & $\ddot{m}$ & $\because$ & $\because$ & $\because$ & $\ddot{*}$ & $\ddot{\ddot{*}}$ & & . . & $\begin{array}{l}x \\
x\end{array}$ & & \\
\hline $\begin{array}{l}\text { - mohrigeformis, Afett........ } \\
\text { - Ottonis, Klotzsch } \ldots . . . .\end{array}$ & 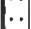 & .. & . & $\therefore$ & $\because$ & $\because$ & $\cdots$ & $\therefore$ & .. & $\cdots$ & $\ldots$ & $\because$ & . & $\cdots$ & $x$ & & \\
\hline - Lindigii, Mett. .... & .. & . & ... & $\cdots$ & .. & .. & $\cdots$ & .. & .. & $\ldots$ & $\therefore$ & .. & ..' & . & $x$ & & \\
\hline - decipiens, Mett. . . . & $\ldots$ & . & . & . & .. & .. & $x$ & & & & & & & & & & \\
\hline - ferruginea, Kunze... & .. & . & ... & . & $\ldots$ & $\cdots$ & . & .. &.. & .. & $\cdots$ & .. & .. . . & $\cdots$ & $x$ & & \\
\hline - Haughtoni, Hook. .. & $\ldots$ & .. & $\ldots$ & 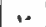 & ... & $\ldots$ & .. & . & $\ldots$ & $x$ & & & & & & & \\
\hline - Hookeri, J. Sm....... & .. & *. & $\ldots$ & $\therefore$ & $\ldots$ & $\cdots$ & .. & $\cdots$ & $\cdots$ & $\cdots$ & $\cdots$ & $\cdots$ & $\ldots$ & . & $x$ & & \\
\hline - Javanicn, Blume .... & $\cdots$ & .. & $x$ & $x$ & $x$ & $x$ & .. & $\cdots$ & $x$ & $\times$ & & & & & & & \\
\hline - Schomburgkiana, Kunze. & 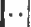 & $\cdots$ & .. & $\cdots$ & $\cdots$ & $\cdots$ & .. & . & .. & $\cdots$ & $\cdots$ & 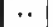 & $\ldots$ & . & $*$ & $x$ & \\
\hline - anreonitens, Hook. . & .. & $\ldots$ & ... & .. & .. & $\cdots$ & .. & .. & .. & $\cdots$ & . & $\cdots$ & . $1 .$. & . & $x$ & & \\
\hline - Matthewsii, Hook. .... & $\ldots$ & -. & . . & $\cdots$ & .. & -. & - & .. & $\cdots$ & $\cdots$ & $\cdots$. & . & $\cdots$ & $\cdots$ & $x$ & & \\
\hline - Warcewiczii, Mett. .... & $\cdots$ & $\because$ & $\cdots$ & $\cdots$ & $\cdots$ & $\cdots$ & $\cdots$ & $\ldots$ & . & $\cdots$ & $\cdots$ & .. & $\cdots 1 \cdots$ & - & $x$ & & \\
\hline - cheilanthoides, $K$ lff, .. & $\cdots$ & $\cdots$ & . & $\cdots$ & $\cdots$ & $\cdots$ & $\cdots$ & $\cdots$ & .. & $x$ & $x$ & & & & & & \\
\hline - flabellata, Hook. , . . . . . & $\cdots$ & . & $\therefore$ & $\cdots$ & $\cdots$ & $\cdots$ & $\cdots$ & $\cdots$ &. & $\cdots$ & $\cdots$ & $\cdots$ & $\ldots \ldots$ & $\cdots$ & $\lambda$ & & \\
\hline - myriophylla, Sw. ... & 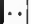 & .. & ... & .. & $\cdots$ & $\cdots$ & .. & .. & .. & $\cdots$ & .. & . & $\cdots \cdots$ & $\cdots$ & * & $x$ & \\
\hline - pedata, Kaulf. ...... & $\cdots$ & . & 1.. & .. & $\cdots$ & * & .. & .. & $\therefore$ & $\because$ & . & $\cdots$ & $\ldots x$ & $x$ & & & \\
\hline - leptophylla, Desv. . & $x$ & $x$ & $x$ & . & $x$ & $\cdots$ & $\cdots$ & $x$ & ... & $x$ & $x$ & . & $\ldots$ & $x$ & $x$ & & \\
\hline - Ascensionis, Houk. . & .. & $\therefore$ & .. & . & .. & . & *. & .. & $\cdots$ & $x$ & & & & & & & \\
\hline - choerophylla, Desv. .. & $\cdots$ & $\cdots$ & .. & 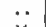 & $\cdots$ & $\cdots$ & -. & . & $\cdots$ & $\because$ & . & $\cdots$ & $\cdots \cdots$ & $x$ & $\cdots$ & $x$ & $x$ \\
\hline - microphylla, Hook. . . & 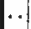 & $\cdots$ & $\cdots$ & $\mathrm{x}$ & & & & & & & & & & & & & \\
\hline - hirta, Desv. ......... & $\cdots$ & - & .. & . & $\cdots$ & $\cdots$ & $\cdots$ & $\cdots$ & ... & $\cdots$ & $\cdots$ & . & $\therefore$ & × & $x$ & & \\
\hline - flexuosa, Desv. ...... & $\cdots$ & - & $\cdots$ & $\cdots$ & $\cdots$ & $\cdots$ & $\cdots$ & & $\cdots$ & $\cdots$ & $\cdots$ & $\cdots$ & $\cdots \cdots$ & $x$ & $x$ & & \\
\hline - Pearcei, Moove ...... & $\cdots$ & & . & $\cdots$ & $\cdots$ & $\cdots$ & .. & - & $\cdots$ & $\therefore$ & . & $\cdots$ & $\ldots$ & $\because$ & $x$ & & \\
\hline - trifoliata, Desv. ..... & $\cdots$ & & $\cdots$ & $\cdots$ & $\cdots$ & $\cdots$ & $\cdots$ & $\cdots$ & $\cdots$ & $\cdots$ & $\cdots$ & $\cdots$ & $\cdots$ & $x$ & $x$ & $x$ & \\
\hline - triangularis, Kaulf. . & $\cdots$ & & $\cdots$ & . & $\cdots$ & $\cdots$ & $\cdots$ & $\cdots$ & $\cdots$ & $\cdots$ & . & $\cdots$ & $\cdots \times$ & $\ddot{x}$ & $x$ & & \\
\hline - tartares, Desv. $\quad \because \ldots$ & {$\left[\begin{array}{l}1 \\
\end{array}\right.$} & .. & $\cdots$ & $\cdots$ & $\cdots$ & $\cdots$ & $\cdots$ & $\cdots$ & $\cdots$ & $\because$ & . & $\cdots$ & $\cdots x$ & $x$ & & & \\
\hline - calomelanos, Kaulf. . & $\cdots$ & . & $\cdots$ & $\cdots$ & . & $\cdots$ & - & . & $x$ & $x$ & . & & $\cdots$ & $x$ & $x$ & $x$ & \\
\hline - pulchella, Lind. ..... & 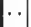 & , & $\cdots$ & $\cdots$ & .. & $\cdots$ & $\cdots$ & $\cdots$ & $\cdots$ & $\cdots$ & . & . & $\cdots$ & $\because$ & $x$ & & \\
\hline - sulphurea, Desv, ... & & $\cdots$ & $\cdots$ & $\cdots$ & $\cdots$ & $\cdots$ & $\cdots$ & & $\cdots$ & $\cdots$ & $\because$ & $\cdots$ & $\cdots \cdot$ & $x$ & $x$ & & \\
\hline - rosea, Desu. .......... & & $\cdots$ & . & $\cdots$ & $\cdots$ & 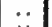 & $\cdots$ & $\because$ & $\cdots$ & $\cdots$ & $x$ & & & & & & \\
\hline - Borneensis, Hook, .... & & $\cdots$ & $\cdots$ & $\cdots$ & $\cdots$ & $x$ & & & & & & & & & & & \\
\hline - cartilagidens, Baker. & $\cdots$ & $\cdots$ & $\cdots$ & $\cdots$ & $\because$ & $x$ & & & & & & & & & & & \\
\hline - obtusifolia, Hook. ... & $\cdots$ & $\cdots$ & $\cdots$ & $\cdots$ & $\because$ & $x$ & & & & & & & & & & & \\
\hline - Lobbiana, Hook. . . & & -. & . & $\because$ & & $\begin{array}{l}x \\
x\end{array}$ & & & & & & & & & & & \\
\hline $\begin{array}{l}\text { - vittifiormis, Hook, }, \cdots \\
\text { - Walliohil, Hook. ,... }\end{array}$ & $1 .$. & $\because$ & $\because$ & $\because$ & $\because$ & $\begin{array}{l}x \\
x\end{array}$ & & & & & & & & & & & \\
\hline - alismafolia, Hook. ' ' ' & $\therefore$ & $\cdots$ & & . & & $x$ & & & & & & & & & & & \\
\hline - quinata, Hook. & & & & & & & & & & & & & & & & & \\
\hline
\end{tabular}




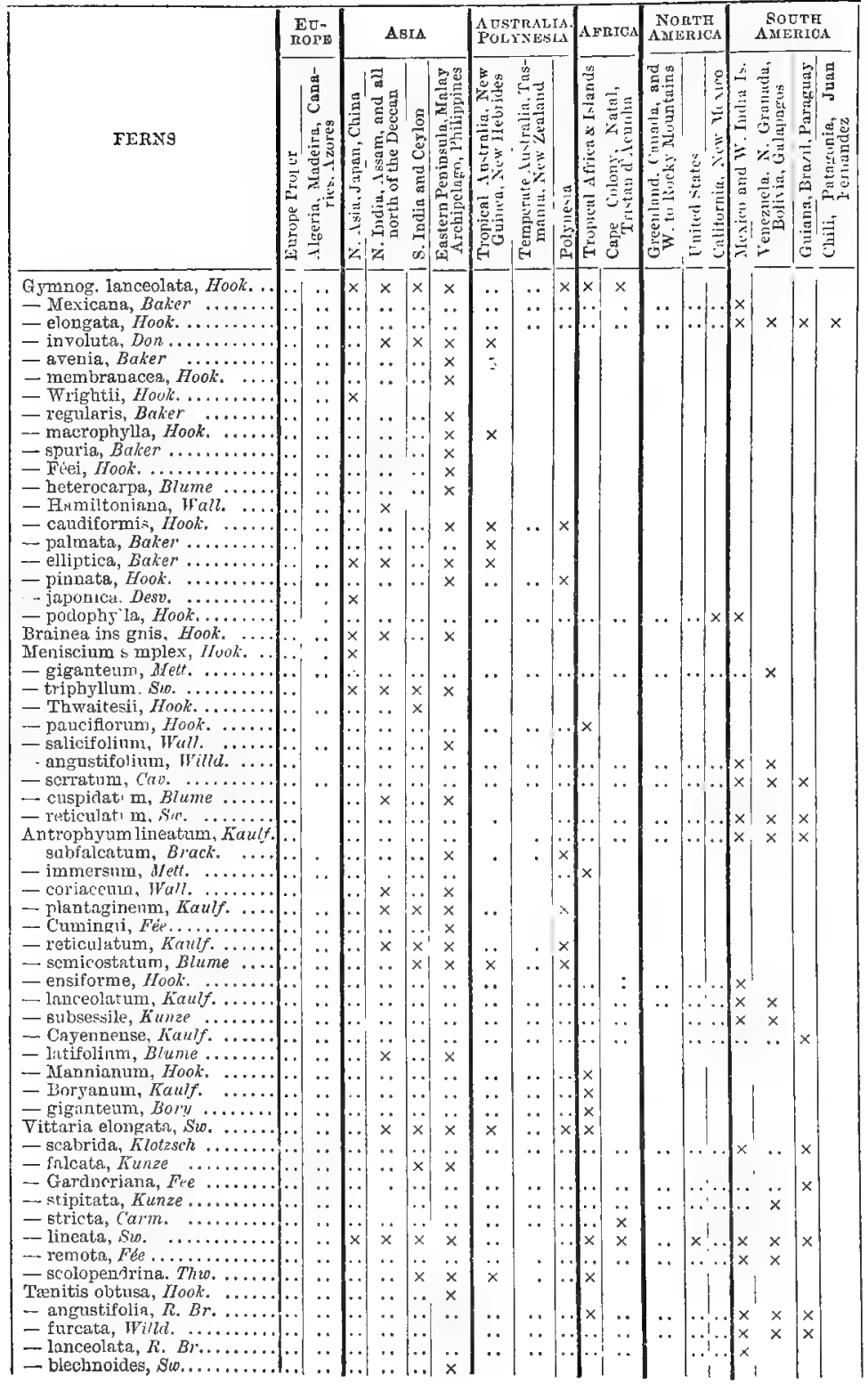




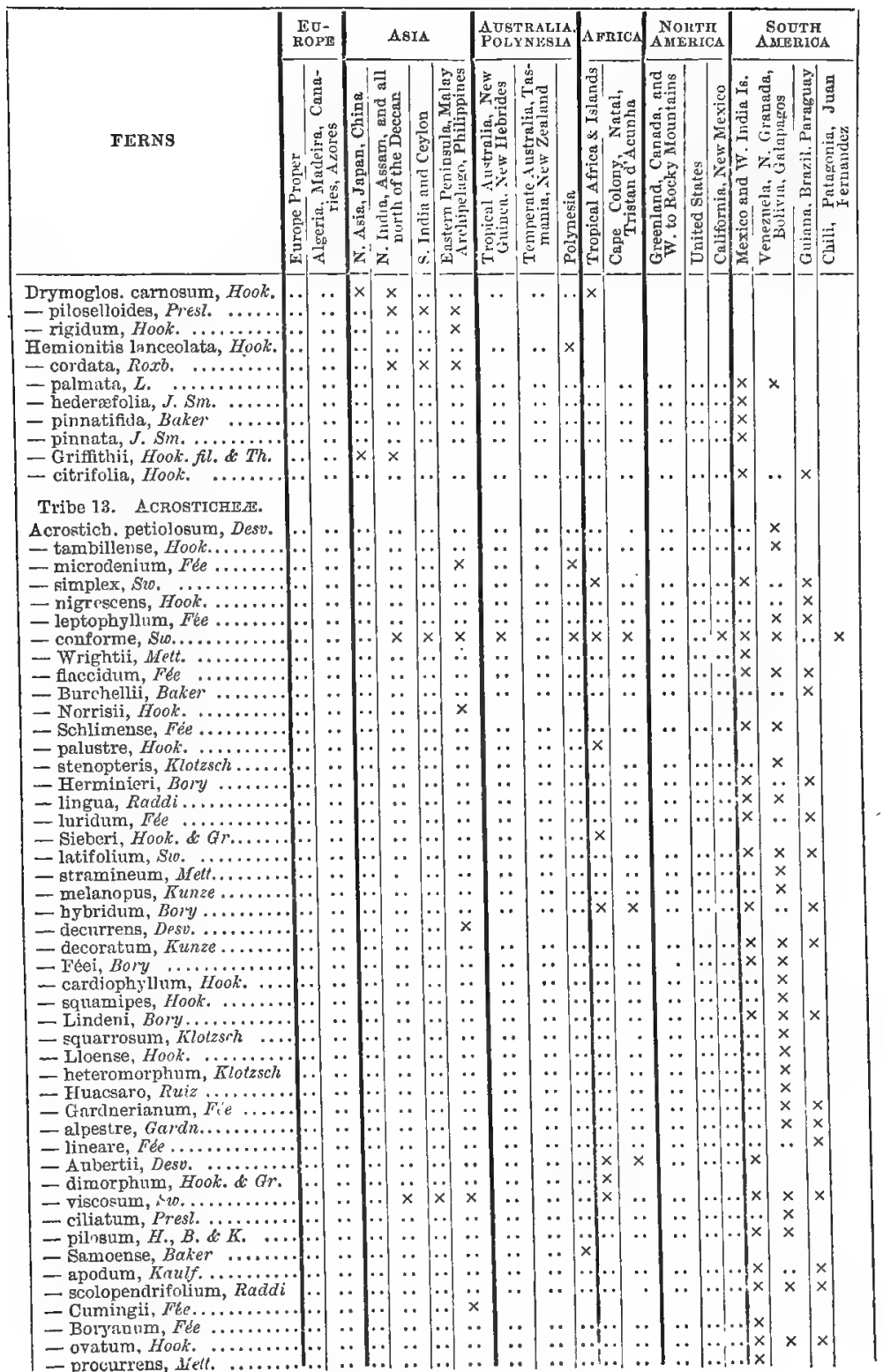




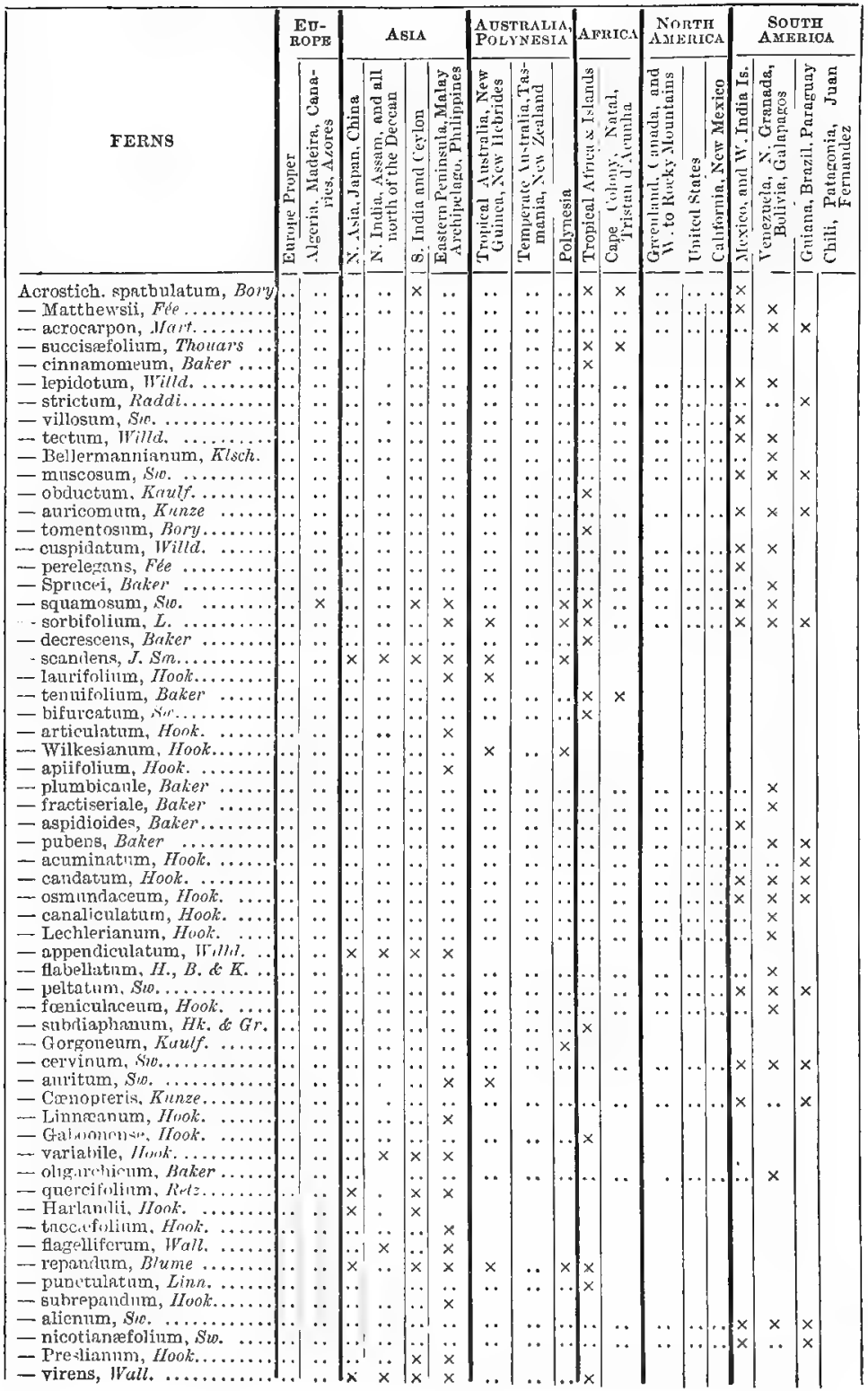




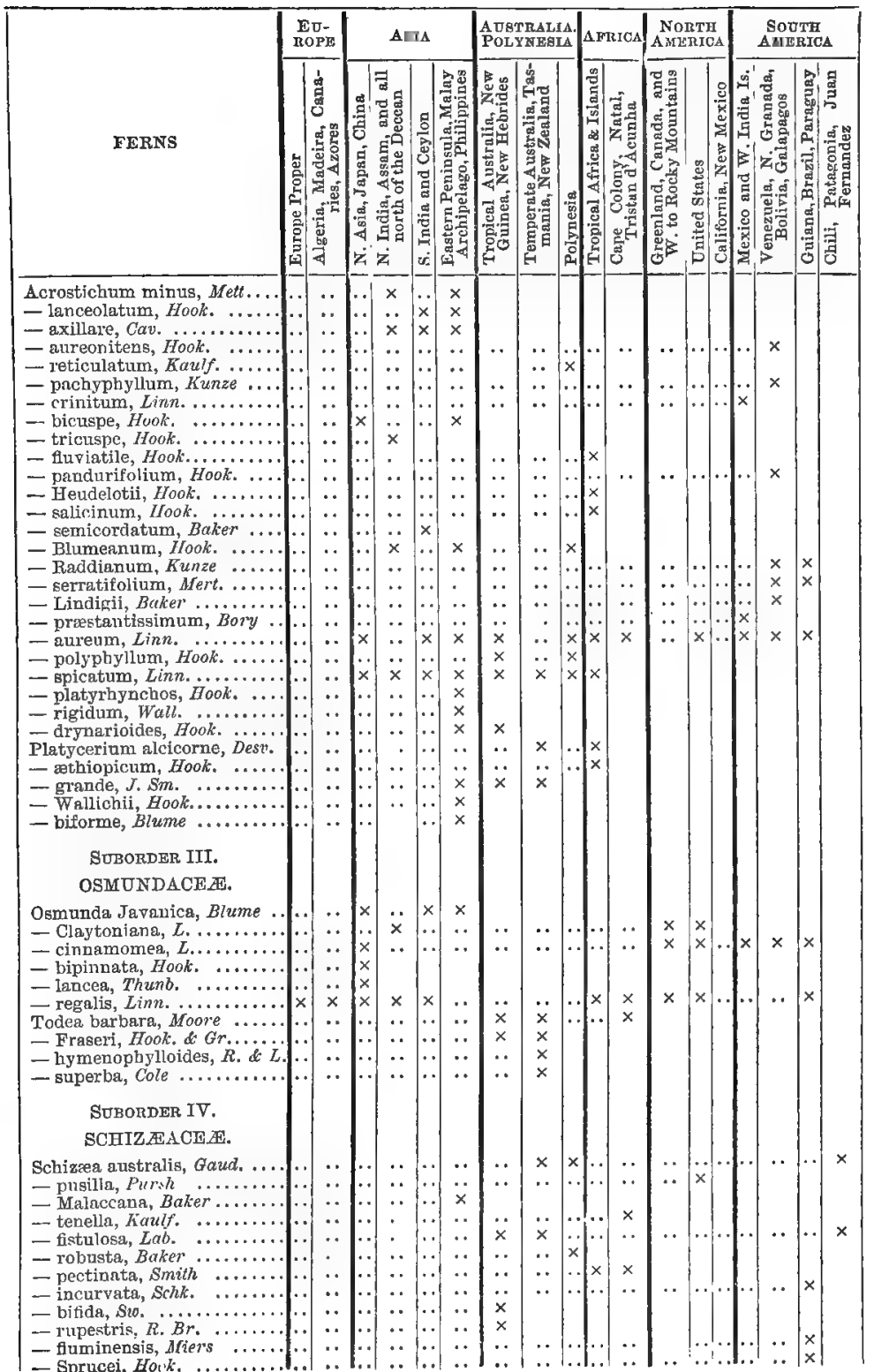




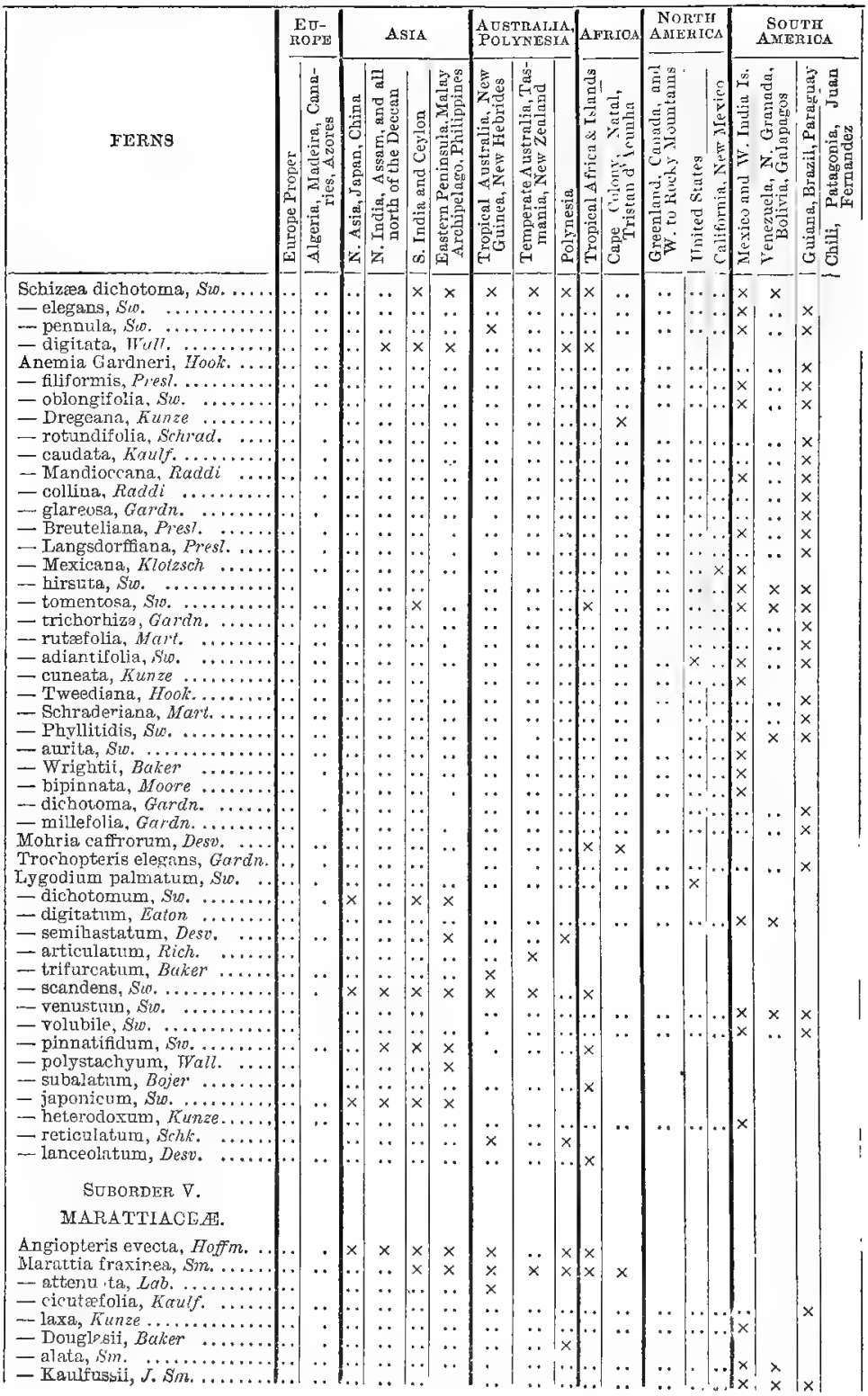




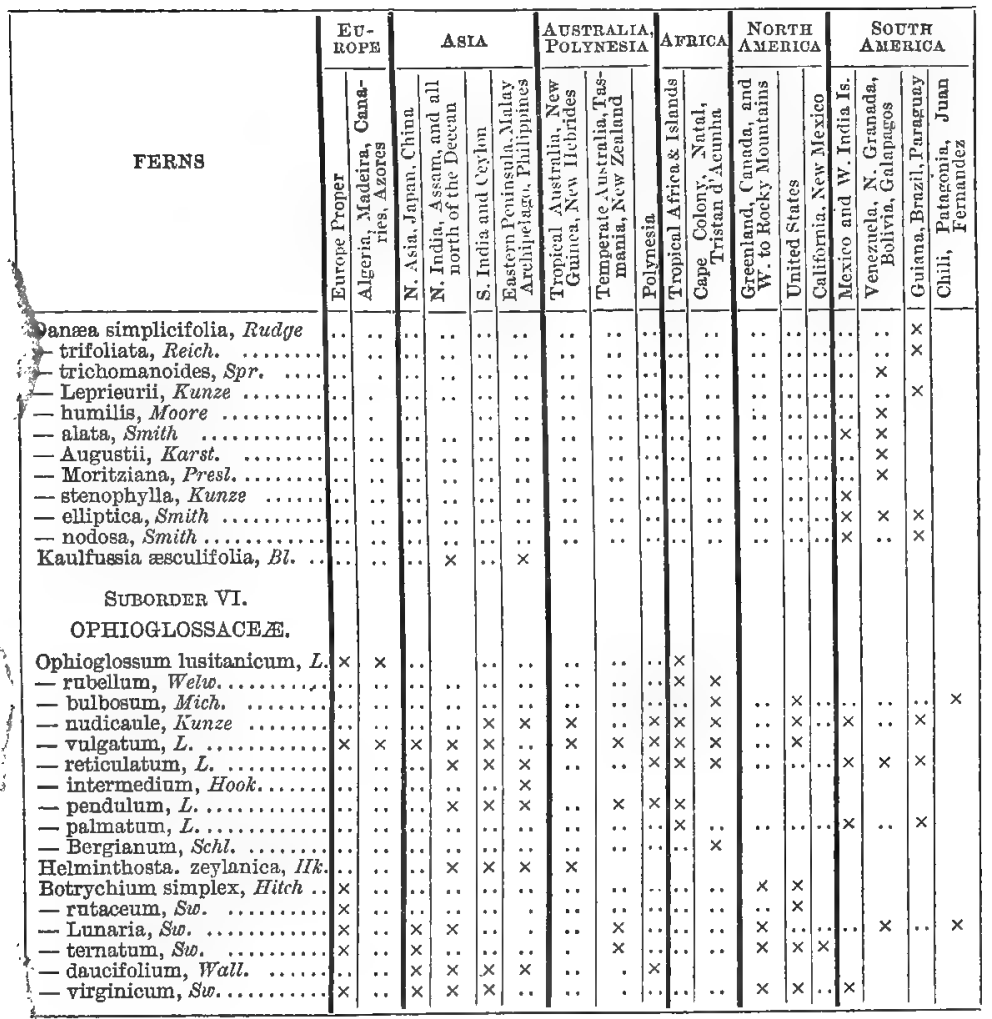

LONDON : PRINTED BY

QPOTRIFWOODE AND CO, NLW-STREBT SQUARB

AND PABLIANRNT STRRIT 



\section{WORKS ON SCIENCE PUBLISHED BY MR. MURRAY.}

A MANUAL OF SCIENTIFIC INQUIRY, for the Use of Travellers. By Various Writers. Edited by STR J. F. Hressereut. 3rd and Retised Edition, by Rev. Robers MaIn, Radcliffe Observer. Post 8vo. 9s.

ELEMENTS OF GEOLOGY; or, The Ancient Changes of the Earth and its Inhabitants, as Illustrated by Geological Monuments. By Sir Charies Lreli, Bart., F.R.S. 7th and entirely Revised Edition. With 700 Woodcuts. 87o. [In preparation.

PRINCIPLES OF GEOLOGY ; or, The Modern Changes of the Earth and its Inhabitants, considered as Hlustrative of Geology. By Sir Chardes Lyerc, Bart. 10th Edition. Woodeuts. 2 vols. 8ro. $32 s$.

THE ANTIQUITY OF MAN, from Geological Evidences; with Remarks on Theories of the Origin of Species by Variation. By SIr Charles Lyele, Bart. 3rd Edition. Illustrations. 8ro. $14 s$.

SILURIA : a History of the Oldest Rocks in the British Isles and other Countries, with a Sketch of the Distribution of Native Gold. By Sir Roderick Murchison, Bart. 4th Edition. Map and Illustrations. 8vo. 30s.

THE CONNECTION OF THE PHYSICAL SCIENCES. By Mary Sonervinde. 9th Edition. Portrait. Post 8ro. $9 s$.

PHYSICAL GEOGRAPHY. By Mary SOMERVIlle. 5th Edition, revised by H. W. Bates. Portrait. Post 8ro. $9 s$.

THE RIVERS, MOUNTAINS, AND SEA-COAST OF YORKSHIRE. With Essays on the Climate, Seenery, and Ancient Inhabitants of the Country. By JoHi Peilirss, F.R.S. 2nd Edition. Plates. 87o. 15s.

THE PHYSICAL GEOGRAPHY OF THE HOLY LAND. By Rev. Eidard Robinson, D.D. Post 8to.

ON MOLECULAR AND MICROSCOPIC SCLENCE. By Mary Somervinte. With 180 Illustrations. 2 vols. post 8vo. $21 s$.

THE ORIGIN OF SPECIES, by Means of Natural Selection; or, the Preservation of Favoured Races in the Struggle for Life. By Charles DARWLN, F.R.S. 5th Edition. Illustrations. Post 87o. 14s. 
Worts on Science, \&.c.

THE VIRIATION OF ANIMALS AND PLINTS UNDER DoNestichtion. By Charles Darwin, F.R.S. Illustrations, 2 vols. 8ro. $28 s$.

THE VARIOUS CONTRIVANCES BY WHICH ORCHIDS ARE FERTILIZED BY INSECTS, and on the Good Effects of Intercrossing. By Charles Dartin, F.R.S. Woodeuts. Post 8ro. $9 s$.

FACTS AND ARGUMENTS FOR DARWIN. By FritZ Mötler. Translated from the German by W. S. Dallas, F.L.S., AssistantSecretary of the Geological Society of London. With Illustrations. Post 8ro. 6s.

A NATURALIST'S VOYAGE ROUND THE WORLD; being a Journal of Researches into the Natural History and Geology of Countries visited. By Charles Darwts, F.R.S. 7th Edition. Illustrations. Post 8ro. $9 s$.

TRAVELS OF A FRENCH NATURALIST IN SIAM, CAMBOdIA, AND LAOS. By HeNri Mounot, F.R.G.S. With 80 Illustrations. 2 rols. 8 ro.

THE NATURALIST ON THE RIVER AMAZONS; Eleven Years of Adventures and Travels. By H.W. Bates. 2nd Edition. Illus. trations. Post 8ro. $12 s$.

TRAVELS IN PERU AND INDIA; while superintending the Collection of Cinchona Plants and Seeds and their Introduction into India. By Clemeats R. MIarkhair. Illustrations. 8vo. $1 \dot{6} s$.

RAMBLES ON THE SHORES AND WATERS OF THE CHINA SEA. Being Obserrations in Natural History during a Voyage to China, Formosa, Borneo, Singapore, \&c., during 1866-67. By Dr. CutheERT CoLungwood. With Illustrations. 8vo. $16 s$.

THE EASTERN ARCHTPELAGO, 1865-66. A Popular Description of the Natural History, Geograplay, Manners, and Customs of the People, and Adrentures among many Tribes, \&c. By Albert S. Bicкmorr, F.R.G.S. With Maps and Illustrations. 8ro. 21s.

THE NILE AND ITS BANKS: a Journal of Travels in Egypt and Nubia, showing their attractions to the Archæologist, Naturalist, and General Tourist. By Rev, A. C. Smiтr, With Woodcuts. 2 vols, post 8 ro. $18 s$.

A MANUAL OF ETHNOLOGY; or, a Popular History of the Races of the Old World. By Chardes L. Brace. 2nd Edition. Post 8ro. $6 s$.

JOHN MURRAY, Albemarle Street. 




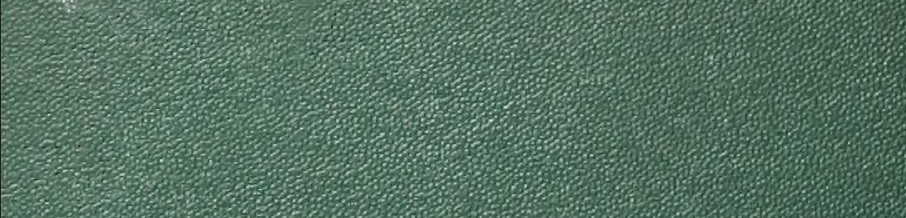

\section{-}

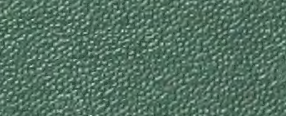

\section{1.}

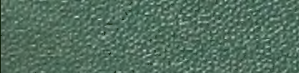

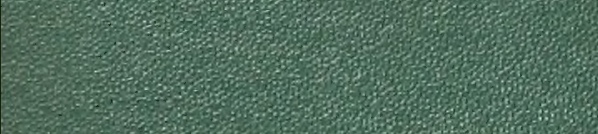

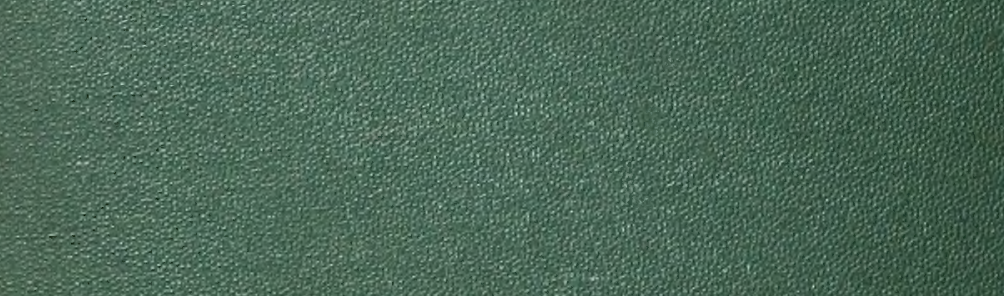
\begin{tabular}{c}
1 \\
\hline
\end{tabular}

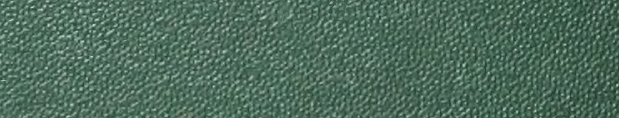

torato

(1)

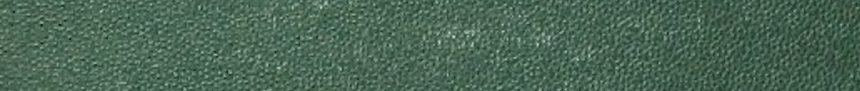

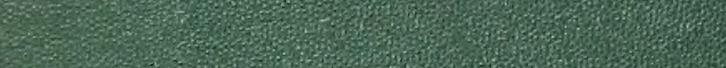

H.

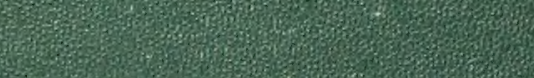

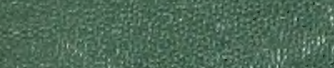

3.

5.7.7.

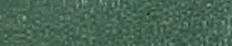

in: 